\title{
Diastereo-, Enantio-, and anti-Selective Formation of Secondary Alcohol and Quaternary Carbon Stereocenters by Cu-catalyzed Additions of B-Substituted Allyl Nucleophiles to Carbonyls
}

\author{
Emilie Wheatley, Joseph M. Zanghi and Simon J. Meek* \\ Department of Chemistry, The University of North Carolina at Chapel Hill, Chapel Hill, North Carolina \\ 27599-3290, United States.
}

SUPPORTING INFORMATION

Table of Contents

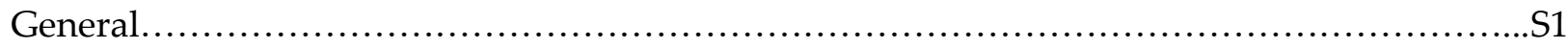

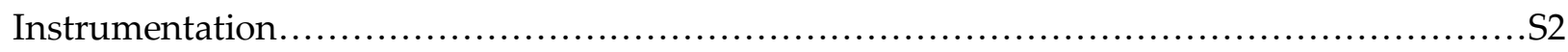

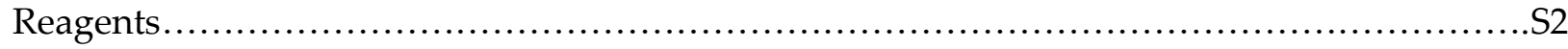

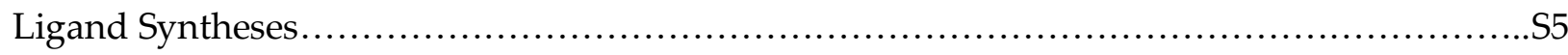

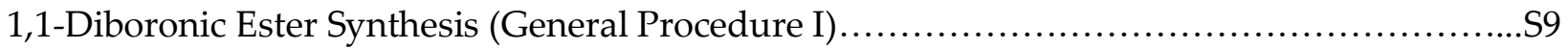

Enantio- and Diastereoselective Allylation of Aldehydes (General Procedure II) ..................S12

Enantio- and Diastereoselective Allylation of Aldehydes (General Procedure III) .................S13

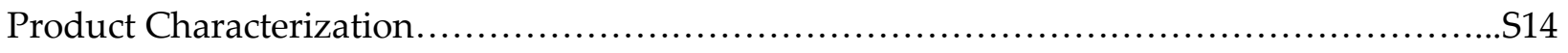

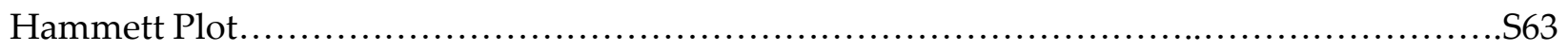

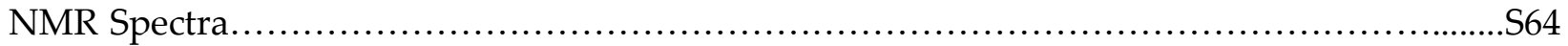

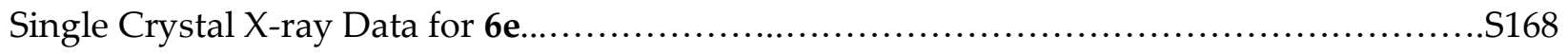

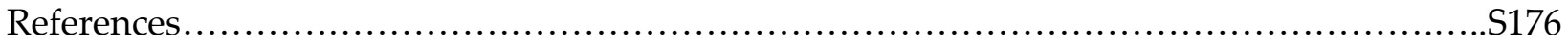

- General: All reactions were carried out in oven-dried $\left(150{ }^{\circ} \mathrm{C}\right)$ or flame-dried glassware under an inert atmosphere of dried $\mathrm{N}_{2}$ unless otherwise noted. Analytical thin-layer chromatography was performed on glass plates coated with $0.25 \mathrm{~mm}$ of $60 \AA$ mesh silica gel. Plates were visualized by exposure to UV light $(254 \mathrm{~nm})$ and/or immersion into $\mathrm{KMnO}_{4}$ or Seebach Stain followed by heating. Column chromatography was performed using silica gel P60 (mesh 230-400) supplied by Silicycle, and refers to flash chromatography unless stated otherwise. Tetrahydrofuran, dichloromethane, diethyl ether, toluene, benzene, and $n$-hexane (OmniSolv) 
were sparged with argon and then purified under a positive pressure of argon through an SG Water, USA Solvent Purification System, through two columns of neutral alumina. The ambient temperature in the laboratory was approximately $22{ }^{\circ} \mathrm{C}$.

- Instrumentation: All ${ }^{1} \mathrm{H}$ NMR spectra were recorded on Bruker Spectrometers (AVANCE600, AVANCE-500 and AVANCE-400). Chemical shifts are reported in ppm from tetramethylsilane and referenced to the residual protio solvent peak $\left(\mathrm{CDCl}_{3}: \delta 7.26\right)$. Data are reported as follows: chemical shift, multiplicity ( $\mathrm{s}=$ singlet, $\mathrm{d}=$ doublet, $\mathrm{t}=$ triplet, $\mathrm{qu}=$ quartet, quint $=$ quintet, $\mathrm{br}=$ broad, $\mathrm{m}=$ multiplet, app = apparent), integration, and coupling constants are given in Hz. ${ }^{13} \mathrm{C}$ NMR spectra were recorded on Bruker Spectrometers (AVANCE-600, and AVANCE-500) with carbon and proton decoupling. Chemical shifts are reported in ppm from tetramethylsilane and referenced to the residual protio solvent peak $\left(\mathrm{CDCl}_{3}: \delta 77.16\right) .{ }^{19} \mathrm{~F} \mathrm{NMR}$ spectra were recorded on Bruker spectrometer (AVANCE-400) with fluorine and proton decoupling. ${ }^{31} \mathrm{P}$ NMR spectra were recorded on Bruker Spectrometers (AVANCE-600, AVANCE500, AVANCE-400). All IR spectra were recorded on a Jasco 260 Plus Fourier transform infrared spectrometer. Specific rotations were determined using a Jasco P1010 polarimeter and concentrations are reported in $\mathrm{g} / 100 \mathrm{~mL}$. Enantiomeric ratios were determined on an Agilent Technologies 1220 Infinity LC using the following columns: Diacel CHIRALPAK IA (4.6 mm x $250 \mathrm{~mm} \times 5 \mu \mathrm{m})$ and Diacel CHIRALPAK IC (4.6 $\mathrm{mm} \times 250 \mathrm{mmL} \times 5 \mu \mathrm{m})$. Alternatively, enantiomeric ratios were determined on a Waters Acquity UPC2 supercritical fluid chromatography system (SFC) using the following columns: Phenomenex Lux Amylose-1 AD (4.6 mm x $150 \mathrm{~mm} \times 5 \mu \mathrm{m}$ ) and Phenomenex Lux Cellulose-1 OD (4.6 mm x $150 \mathrm{~mm} \times 5 \mu \mathrm{m}$ ). All HPLC samples were separated at $22^{\circ} \mathrm{C}$, and all SFC samples were separated at $30{ }^{\circ} \mathrm{C}$. Racemic products were obtained using rac-L5. Please note, differences in $\mathrm{dr}$ between some of the racemic and enantioenriched HPLC/SFC chromatograms are the result of uncatalyzed background addition that occurred during preparation of the racemic samples. High resolution mass spectrometry samples were analyzed with a ThermoScientific $Q$ Exactive HF-X mass spectrometer. Samples were introduced via an atmospheric pressure chemical ionization (APCI) source at a flow rate of $20 \mu \mathrm{L} / \mathrm{min}$ (samples in 100\% MeOH) or via a micro-electrospray source at a flow rate of $10 \mu \mathrm{L} / \mathrm{min}(\mathrm{MeOH})$. Xcalibur (ThermoFisher, Breman, Germany) was used to analyze the data. Absolute stereochemistry was assigned by single crystal $X$-ray crystallography of (6e) via anomalous scattering. X-ray sample was acquired using $\mathrm{Cu} \mathrm{K} \alpha(\lambda=1.54178 \AA$ ) radiation. All melting points were measured on powdered solids using a Mel-Temp II capillary melting point device and are uncorrected unless otherwise stated.

\section{- Reagents:}


4-Anisaldehyde was purchased from Alfa Aesar, dried over $\mathrm{CaH}_{2}$, distilled under reduced pressure and then sparged with dry $\mathrm{N}_{2}$.

Benzaldehyde was purchased from Alfa Aesar, dried over $\mathrm{CaH}_{2}$, distilled under reduced pressure and then sparged with dry $\mathrm{N}_{2}$.

Benzo[b]thiophene-2-carboxaldehyde was purchased from Alfa Aesar and was dried by azeotropic distillation.

2-Benzofurancarboxaldehyde was purchased from Alfa Aesar and was dried by azeotropic distillation.

Bis(pinacolato)diboron $\left(\mathbf{B}_{2}(\text { pin })_{2}\right)$ was purchased from Frontier Scientific, recrystallized from boiling hexanes, azeotropically dried three times with benzene, and stored in a $\mathrm{N}_{2}$-filled glovebox. 1-Boc-piperidine-4-carboxaldehyde was purchased from Oakwood and was dried by azeotropic distillation.

4-Bromobenzaldehyde was purchased from Alfa Aesar, dried over $\mathrm{CaH}_{2}$, distilled under reduced pressure and then sparged with dry $\mathrm{N}_{2}$.

2-Bromo-4-chlorobenzaldehyde was purchased from Alfa Aesar and was dried by azeotropic distillation.

Chloroform-d was purchased from Cambridge Isotope Laboratories and used without further purification.

Copper(I) tert-butoxide was prepared according to literature procedure ${ }^{1}$ and at $-30{ }^{\circ} \mathrm{C}$ in a $\mathrm{N}_{2}$ filled glovebox.

Cyclohexanecarboxaldehyde was purchased from Alfa Aesar, dried over $\mathrm{CaH}_{2}$, distilled under reduced pressure and then sparged with dry $\mathrm{N}_{2}$.

1-Cyclohexene-1-carboxaldehyde was purchased from Alfa Aesar, dried over $\mathrm{CaH}_{2}$, distilled under reduced pressure and then sparged with dry $\mathrm{N}_{2}$.

Cyclopropanecarboxaldehyde was purchased from Alfa Aesar, dried over $\mathrm{CaH}_{2}$, distilled under reduced pressure and then sparged with dry $\mathrm{N}_{2}$.

2,6-Dichloropyridine-3-carboxaldehyde was purchased from Ark Pharm, dried over $\mathrm{CaH}_{2}$, distilled under reduced pressure and then sparged with dry $\mathrm{N}_{2}$.

4-Fluorobenzaldehyde was purchased from Sigma-Aldrich, dried over $\mathrm{CaH}_{2}$, distilled under reduced pressure and then sparged with dry $\mathrm{N}_{2}$.

4-Formylbenzonitrile was purchased from Matrix and was dried by azeotropic distillation.

Furfural was purchased from Alfa Aesar, dried over $\mathrm{CaH}_{2}$, distilled under reduced pressure and then sparged with dry $\mathrm{N}_{2}$.

Hexamethyldisiloxane was purchased from Alfa Aesar and stored over $4 \AA$ molecular sieves.

1-hexyne was purchased from Alfa Aesar and used without purification.

Iodine was purchased from Fisher and used without purification.

Isovaleraldehyde was purchased from Alfa Aesar, dried over $\mathrm{CaH}_{2}$, distilled under reduced pressure and then sparged with dry $\mathrm{N}_{2}$. 
Methanol was purchased from VWR Life Science, dried over $\mathrm{Mg} / \mathrm{I}$, distilled, and stored over 3 $\AA$ A molecular sieves under $\mathrm{N}_{2}$.

Methanol- $d 4$ was purchased from Cambridge Isotopes and used without further purification.

Methyl 4-formylbenzoate was purchased from Ark Pharm and was dried by azeotropic distillation.

n-Butyllithium was purchased from Strem and used without further purification.

3-Nitrobenzaldehyde was purchased from TCI and was dried by azeotropic distillation.

4-Nitrobenzaldehyde was purchased from Alfa Aesar and was dried by azeotropic distillation.

Palladium (II) acetate $\left(\mathbf{P d}(\mathrm{OAc})_{2}\right)$ was purchased from Strem and stored in a $\mathrm{N}_{2}$-filled glovebox.

4-Pentenal was purchased from Alfa Aesar, dried over $\mathrm{CaH}_{2}$, distilled under reduced pressure and then sparged with dry $\mathrm{N}_{2}$.

Phenyl propargyl aldehyde was purchased from Alfa Aesar, dried over $\mathrm{CaH}_{2}$, distilled under reduced pressure and then sparged with dry $\mathrm{N}_{2}$.

Pivalaldehyde was purchased from Alfa Aesar, dried over $\mathrm{CaH}_{2}$, distilled under reduced pressure and then sparged with dry $\mathrm{N}_{2}$.

Potassium carbonate was purchased from VWR Life Science and used without addition purification.

Potassium tert-butoxide was purchased from Strem and stored in a $\mathrm{N}_{2}$-filled glovebox.

(1R)-(-)-Myrtenal was purchased from ACROS, dried over $\mathrm{CaH}_{2}$, distilled under reduced pressure and then sparged with dry $\mathrm{N}_{2}$.

(S)-(-)-Citronellal was purchased from TCI, dried over $\mathrm{CaH}_{2}$, distilled under reduced pressure and then sparged with dry $\mathrm{N}_{2}$.

Sodium hydride $(60 \%$ dispersion in mineral oil) was purchased from Sigma-Aldrich and used without further purification.

tert-Butyl alcohol was purchased from Alfa Aesar, dried over $\mathrm{MgSO}_{4}$, filtered, dried overnight over $\mathrm{CaH}_{2}$ at $30{ }^{\circ} \mathrm{C}$, distilled, and stored over $4 \AA$ molecular sieves under $\mathrm{N}_{2}$.

tert-Butyl 3-formyl-1H-indole-1-carboxylate was purchased from Sigma Aldrich and was dried by azeotropic distillation.

2-Thiophenecarboxaldehyde was purchased from Alfa Aesar, dried over $\mathrm{CaH}_{2}$, distilled under reduced pressure and then sparged with dry $\mathrm{N}_{2}$.

trans-2-Methyl-2-butenal was purchased from Alfa Aesar, dried over $\mathrm{CaH}_{2}$, distilled under reduced pressure and then sparged with dry $\mathrm{N}_{2}$.

trans- $\alpha$-Methylcinnamaldehyde was purchased from Alfa Aesar, dried over $\mathrm{CaH}_{2}$, distilled under reduced pressure and then sparged with dry $\mathrm{N}_{2}$.

Trimethylaluminum (2.0 $\mathrm{M}$ in hexanes) was purchased from Sigma-Aldrich, stored at $0^{\circ} \mathrm{C}$, and used without purification.

Zirconocene dichloride was purchased from Strem and stored in a $\mathrm{N}_{2}$-filled glovebox. 
- Ligand synthesis

2,6-bis(3,5-di-tert-butylphenyl)-N,N-dimethyl-8,9,10,11,12,13,14,15-octahydrodinaphtho[2,1d:1',2'-f][1,3,2]dioxaphosphepin-4-amine (L4)

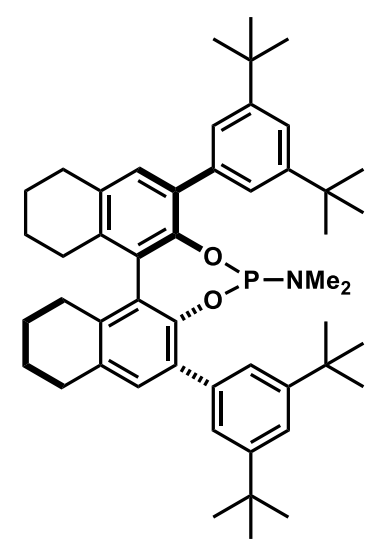

(L4)

Phosphoramidite (L4) was prepared according to a literature procedure².

2,6-bis(3,5-dimethylphenyl)-N,N-dimethyl-8,9,10,11,12,13,14,15-octahydrodinaphtho[2,1d:1',2'-f][1,3,2]dioxaphosphepin-4-amine (L5)<smiles></smiles>

(L5)

Phosphoramidite (L5) was prepared according to a literature procedure ${ }^{3}$.

2,6-bis(3,5-bis(trifluoromethyl)phenyl)-N,N-dimethyl-8,9,10,11,12,13,14,15octahydrodinaphtho[2,1-d:1',2'-f][1,3,2]dioxaphosphepin-4-amine (L6) 


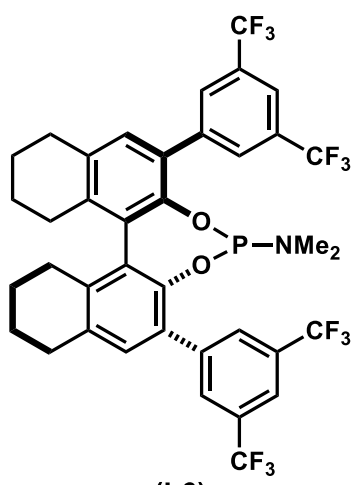

(L6)

Phosphoramidite (L6) was prepared according to a literature procedure ${ }^{4}$

1,3-diisopropyl-2-methoxybenzene (S1)

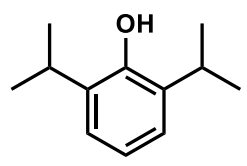

2,6-diisopropylphenol

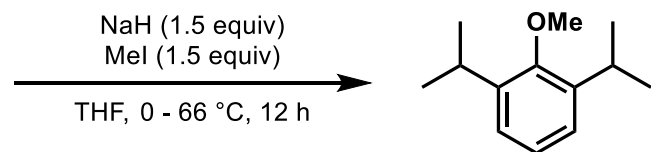

(S1)

2,6-diisopropylphenol (5.20 g, $29 \mathrm{mmol}, 1.0$ equiv) was weighed into an oven-dried round bottom flask charged with magnetic stirrer bar, dissolved in THF (140 mL, 0.20 M) and cooled to $0{ }^{\circ} \mathrm{C}$ in an ice bath. Sodium hydride $(1.80 \mathrm{~g}, 44 \mathrm{mmol}, 1.5 \mathrm{equiv}, 60 \% \mathrm{Wt}$ in mineral oil $)$ was added slowly and allowed to stir for 5 minutes before adding iodomethane ( $2.7 \mathrm{~mL}, 44 \mathrm{mmol}, 1.5$ equiv) at $0{ }^{\circ} \mathrm{C}$. The reaction mixture was allowed to warm to room temperature and stirred for 1 hour before heating to $66^{\circ} \mathrm{C}$ (mineral oil bath) and refluxed with a condenser for another 12 hours.

Upon completion, the reaction was allowed to cool to room temperature and was quenched with water. The reaction mixture was extracted with diethyl ether $(3 \times 100 \mathrm{~mL})$. The organic layers were combined and dried with $\mathrm{MgSO}_{4}$, filtered through cotton and concentrated under vacuum. The crude material was purified by silica gel flash column chromatography (40:1 -> 10:1 hexanes/ethyl acetate) affording the product (5.20 g, 93\% yield) as a pale-yellow oil.

${ }^{1} \mathbf{H}$ NMR $\left(600 \mathrm{MHz}, \mathrm{CDCl}_{3}\right) \delta 7.13$ - $7.09(\mathrm{~m}, 3 \mathrm{H}), 3.75(\mathrm{~s}, 3 \mathrm{H}), 3.43$ - 3.28 (hept, $\left.6.9 \mathrm{~Hz}, 2 \mathrm{H}\right), 1.30$ $1.16(\mathrm{~d}, 6.9 \mathrm{~Hz}, 12 \mathrm{H}) .{ }^{13} \mathrm{C}$ NMR $\left(151 \mathrm{MHz}, \mathrm{CDCl}_{3}\right) \delta$ 154.6, 141.8, 124.6, 124.2, 62.4, 26.6, 24.2. HRMS (m/z) calc. for [M+H'] $\mathrm{C}_{13} \mathrm{H}_{21} \mathrm{O}^{+}: 193.1587$ found: 193.1588. IR (cm-1) 2963 (s), 2929 (s), 2870 (m), 1459 (m), 1327 (w), 1255 (m), 1201 (m) 1014 (m), 764 (m), 739 (w).

\section{2-(3,5-diisopropyl-4-methoxyphenyl)-4,4,5,5-tetramethyl-1,3,2-dioxaborolane (S2)}


<smiles>COc1c(C(C)C)cccc1C(C)C</smiles>

(S1)

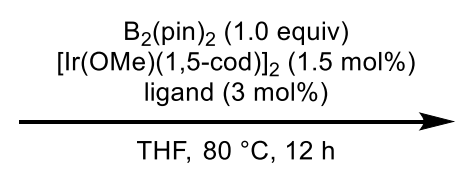

$\mathrm{THF}, 80^{\circ} \mathrm{C}, 12 \mathrm{~h}$

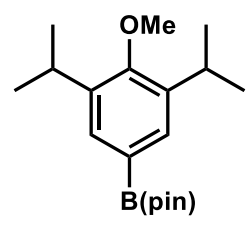

(S2)

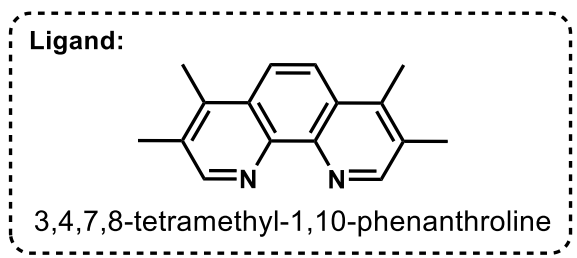

In a $\mathrm{N}_{2}$-filled glovebox, $[\operatorname{Ir}(\mathrm{OMe})(1,5-\mathrm{cod})]_{2}(0.13 \mathrm{~g}, 0.20 \mathrm{mmol}, 1.5 \mathrm{~mol} \%)$ and 3,4,7,8tetramethyl-1,10-phenanthroline (92 $\mathrm{mg}, 0.39 \mathrm{mmol}, 3.0 \mathrm{~mol} \%$ ) were weighed into a round bottom flask charged with magnetic stirrer bar. THF $(29 \mathrm{~mL})$ was added and the reaction mixture was allowed to stir for 10 minutes. In a separate round bottom flask, $\mathrm{B}_{2}$ (pin)2 $(3.3 \mathrm{~g}, 13 \mathrm{mmol}, 1.0$ equiv) was weighed and the catalyst mixture was transferred to this flask before sealing with a septum and electrical tape and removing from the glovebox. S1 (5.0 g, 26 mmol, 2.0 equiv) was added neat and the reaction was allowed to stir at $80^{\circ} \mathrm{C}$ (mineral oil bath) with a reflux condenser for 12 hours.

Upon completion, the reaction was allowed to cool to room temperature and was quenched with saturated aqueous $\mathrm{NH}_{4} \mathrm{Cl}(30 \mathrm{~mL})$ before extracting with diethyl ether $(3 \times 30 \mathrm{~mL})$. The organic layers were combined and dried with $\mathrm{MgSO}_{4}$, filtered through cotton and concentrated under vacuum. The crude material was purified by silica gel flash column chromatography (30:1 $\rightarrow$ 10:1 hexanes/ethyl acetate) to obtain the product $(7.0 \mathrm{~g}, 84 \%$ yield) as a white solid (melting point $=132-134^{\circ} \mathrm{C}$ ).

${ }^{1} \mathrm{H}$ NMR (600 MHz, $\left.\mathrm{CDCl}_{3}\right) \delta 7.57$ (s, 2H), 3.74 (s, 3H), 3.40 - 3.25 (hept, $\left.6.9 \mathrm{~Hz}, 2 \mathrm{H}\right), 1.34$ (s, 12H), $1.26(\mathrm{~d}, 6.9 \mathrm{~Hz}, 12 \mathrm{H}) .{ }^{13} \mathrm{C}$ NMR $\left(151 \mathrm{MHz}, \mathrm{CDCl}_{3}\right) \delta 157.5,141.1,131.2,83.7,62.3,26.6,25.0,24.2$. HRMS (m/z) calc. for [M+H'] $\mathrm{C}_{19} \mathrm{H}_{32} \mathrm{BO}_{3}{ }^{+}: 319.2439$ found: 319.2440. IR (cm $\left.{ }^{-1}\right) 2960$ (s), 2929 (s), 2869 (m), 1604 (s), 1462 (m), 1375 (s), 1309 (s), 1145 (m), 1008 (s), 852 (w), 740 (s), 693 (m).

\section{3,3'-bis(3,5-diisopropyl-4-methoxyphenyl)-5,5',6,6',7,7',8,8'-octahydro-[1,1'-binaphthalene]-}

\section{2,2'-diol (S3)}

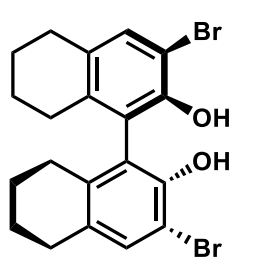

(1.0 equiv)

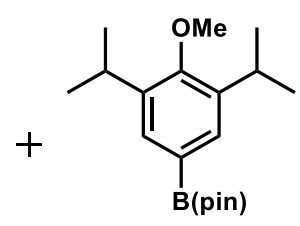

(S2)

(3.0 equiv)<smiles>COc1c(C(C)C)cc(-c2cc3c(c(-c4c(O)c(-c5cc(C(C)C)c(OC)c(C(C)C)c5)cc5c4CCCC5)c2O)CCCC3)cc1C(C)C</smiles>

(S3) 
In a $\mathrm{N}_{2}$-filled glovebox, $\mathrm{Pd}(\mathrm{OAc}) 2(3.0 \mathrm{mg}, 0.0133 \mathrm{mmol}, 4 \mathrm{~mol} \%)$ and CataCXium A (5.9 mg, $0.0166 \mathrm{mmol}, 5 \mathrm{~mol} \%$ ) were weighed into an $8 \mathrm{~mL}$ vial charged with magnetic stirrer bar. 1,2dimethoxyethane (DME) $(1.65 \mathrm{~mL})$ was added and the vial was sealed with a Teflon-lined septa cap and removed from the glovebox. In another $8 \mathrm{~mL}$ vial, $(R)-\mathrm{H}_{8}-3,3^{\prime}-\mathrm{Br}^{-\mathrm{BINOL}^{5}}$ (150.0 mg, $0.3317 \mathrm{mmol}, 1.0$ equiv) and aryl boronic ester (S2) $(235.0 \mathrm{mg}, 0.9951 \mathrm{mmol}, 3.0$ equiv) were weighed and magnetic stirrer bar added. This vial was evacuated and backfilled with $\mathrm{N}_{2}$. The $(R)$ $\mathrm{H}_{8}-3,3$ '-Br-BINOL/boronic acid were suspended in DME (1.65 mL). A N2-sparged solution of aqueous $\mathrm{K}_{2} \mathrm{CO}_{3}(1.65 \mathrm{~mL}, 1 \mathrm{M}, 5.0$ equiv) was added to the resulting suspension. Lastly, the catalyst mixture was transferred by syringe to the $(R)-\mathrm{H}_{8}-3,3^{\prime}-\mathrm{Br}-\mathrm{BINOL} /$ boronic acid. The biphasic mixture was stirred vigorously at $100{ }^{\circ} \mathrm{C}$ (mineral oil bath or aluminum reaction block) for $18 \mathrm{~h}$. The reaction was then cooled to room temperature. The organic layer was removed, and the aqueous layer was extracted three times with DCM $(3 \times 1 \mathrm{~mL})$. The organic layers were combined, dried over $\mathrm{MgSO}_{4}$, and concentrated by rotary evaporation. The crude material was purified by silica gel flash column chromatography (40:1 -> 10:1 hexanes/diethyl ether) to obtain BINOL (S3) $\left(140.0 \mathrm{mg}\right.$, 94\% yield) as a white solid (melting point $=120-122^{\circ} \mathrm{C}$ ).

${ }^{1} \mathrm{H}$ NMR $\left(600 \mathrm{MHz}, \mathrm{CDCl}_{3}\right) \delta 7.31$ (s, 4H), $7.12(\mathrm{~s}, 2 \mathrm{H}), 5.03(\mathrm{~s}, 2 \mathrm{H}), 3.77(\mathrm{~s}, 6 \mathrm{H}), 3.41-3.33(\mathrm{~m}, 4 \mathrm{H})$, $2.93-2.77(\mathrm{~m}, 4 \mathrm{H}), 2.48-2.38(\mathrm{~m}, 2 \mathrm{H}), 2.31-2.22(\mathrm{~m}, 2 \mathrm{H}), 1.83-1.75(\mathrm{~m}, 4 \mathrm{H}), 1.75-1.69(\mathrm{~m}, 4 \mathrm{H})$, 1.30 - $1.24(\mathrm{~m}, 24 \mathrm{H}) .{ }^{13} \mathrm{C}$ NMR $\left(151 \mathrm{MHz}, \mathrm{CDCl}_{3}\right) \delta$ 153.8, 148.0, 141.8, 136.3, 133.9, 131.5, 130.1, $126.4,125.2,120.7,62.4,30.4,29.9,29.4,27.3,26.7,24.3,24.3,23.2,23.2$. HRMS (m/z) calc. for $\left[\mathrm{M}+\mathrm{H}^{+}\right] \mathrm{C}_{46} \mathrm{H}_{59} \mathrm{O}_{4}{ }^{+}: 675.4408$ found: 675.4406. IR $\left(\mathrm{cm}^{-1}\right) 3519(\mathrm{w}), 2962(\mathrm{w}), 2930(\mathrm{w}), 1458(\mathrm{w}), 1265$ (s), $1011(\mathrm{w}), 739(\mathrm{~s}), 706(\mathrm{w}) .[\alpha]_{\mathrm{D}^{23}}=-3.9\left(\mathrm{c}=0.75, \mathrm{CH}_{2} \mathrm{Cl}_{2}, \mathrm{l}=1 \mathrm{dm}\right)$.

\section{2,6-bis(3,5-diisopropyl-4-methoxyphenyl)-N,N-dimethyl-8,9,10,11,12,13,14,15- octahydrodinaphtho[2,1-d:1',2'-f][1,3,2]dioxaphosphepin-4-amine (L7)}

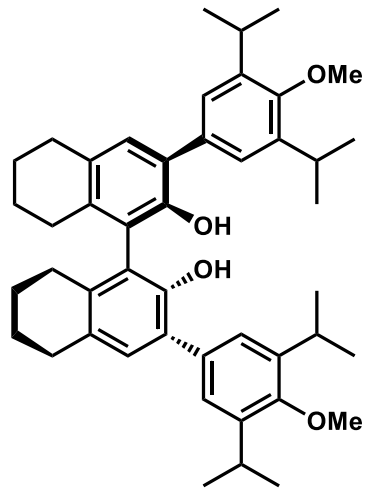

(S3)

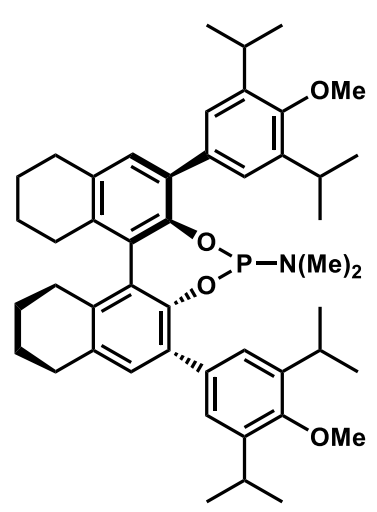

(L7)

H8-BINOL derivative (S3) (500.0 mg, $0.741 \mathrm{mmol}, 1.0$ equiv) was weighed into an $8 \mathrm{~mL}$ vial charged with magnetic stir bar. The vial was sealed with a Teflon-lined septa cap and evacuated 
and backfilled with $\mathrm{N}_{2}$ before adding dry toluene $(4.0 \mathrm{~mL}, 0.18 \mathrm{M}$ solution in S3). Tris(dimethylamino)phosphine (133.0 mg, $0.815 \mathrm{mmol}, 1.1$ equiv) was added via microsyringe. The vial was flushed with argon and the septa cap replaced quickly with a hard, plastic cap. The reaction was heated to $110{ }^{\circ} \mathrm{C}$ (mineral oil bath or aluminum reaction block) and allowed to stir for $4 \mathrm{~h}$. Upon completion, the reaction was cooled to room temperature and concentrated by rotary evaporation. The crude material was purified by silica gel flash column chromatography (40:1 -> 10:1 hexanes/ethyl acetate) to obtain the phosphoramidite L7 (476.0 $\mathrm{mg}, 86 \%$ yield) as a white solid (melting point $=138-142^{\circ} \mathrm{C}$ ).

${ }^{1} \mathbf{H}$ NMR (600 MHz, $\left.\mathrm{CDCl}_{3}\right) \delta 7.41-7.37$ (d, $\left.7.3 \mathrm{~Hz}, 4 \mathrm{H}\right), 7.19-7.14$ (d, $\left.13.9 \mathrm{~Hz}, 2 \mathrm{H}\right), 3.75$ (s, 3H), $3.74(\mathrm{~s}, 3 \mathrm{H}), 3.43-3.36(\mathrm{~m}, 2 \mathrm{H}), 3.35-3.28(\mathrm{~m}, 2 \mathrm{H}), 2.96-2.82(\mathrm{~m}, 4 \mathrm{H}), 2.76-2.66(\mathrm{~m}, 2 \mathrm{H}), 2.47$ $2.41(\mathrm{~m}, 1 \mathrm{H}), 2.40-2.34(\mathrm{~m}, 1 \mathrm{H}), 1.90(\mathrm{~s}, 3 \mathrm{H}), 1.89(\mathrm{~s}, 3 \mathrm{H}), 1.87-1.79(\mathrm{~m}, 6 \mathrm{H}), 1.73-1.66(\mathrm{~m}, 1 \mathrm{H})$, 1.65 - $1.59(\mathrm{~m}, 1 \mathrm{H}), 1.32-1.29(\mathrm{~m}, 6 \mathrm{H}), 1.26-1.19(\mathrm{~m}, 18 \mathrm{H}) .{ }^{13} \mathrm{C}$ NMR $\left(151 \mathrm{MHz}, \mathrm{CDCl}_{3}\right) \delta 153.6$, $153.3,145.5,145.1,140.9,136.8,134.6,134.0,133.8,133.3,131.8,131.3,130.5,130.5,130.4,130.1$, 129.4, 125.9, 62.5, 62.4, 34.4, 34.2, 29.5, 29.4, 28.0, 26.7, 26.6, 24.4, 24.3, 24.2, 24.2, 23.2, 23.1, 23.1, 22.9. ${ }^{31} \mathbf{P}$ NMR $\left(243 \mathrm{MHz}_{1} \mathrm{CDCl}_{3}\right) \delta$ 139.7. HRMS (m/z) calc. for $\left[\mathrm{M}+\mathrm{H}^{+}\right] \mathrm{C}_{48} \mathrm{H}_{63} \mathrm{NO}_{4} \mathrm{P}^{+}: 748.4489$ found: 748.4478. IR (cm-1) 2961 (s), 2931 (s), 2869 (w), 1458 (w), 1308 (w), 1265 (m), 1013 (m), 740 (s), $704(\mathrm{~m}) .[\alpha]_{\mathrm{D}^{23}}=-344.3\left(\mathrm{c}=0.9, \mathrm{CH}_{2} \mathrm{Cl}_{2}, 1=1 \mathrm{dm}\right)$.

\section{- General Procedure (I) for the synthesis of 1,1-Diboronic Esters}
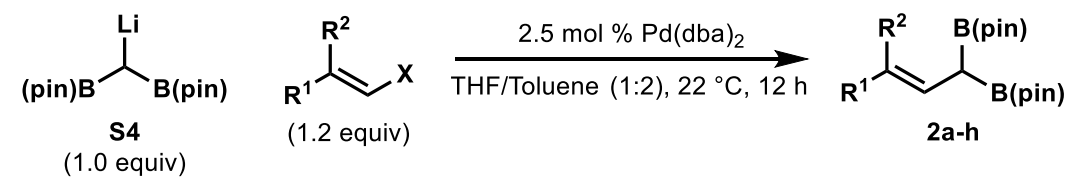

As in our previous report ${ }^{3}$, in a $\mathrm{N}_{2}$-filled glovebox, lithiated diborylmethane (Li-DBM, S4) (1.0 equiv) was weighed into a round bottom flask charged with a magnetic stir bar. To a separate round bottom flask, $\mathrm{Pd}(\mathrm{dba})_{2}(2.5 \mathrm{~mol} \%)$ was added. The flasks were capped with septa, sealed with electrical tape and removed from the glovebox. Toluene was added to the $\mathrm{Pd}(\mathrm{dba})_{2}$ giving a $12.5 \mathrm{mM}$ solution. THF was added to the Li-DBM to generate a suspension $(2 \mathrm{~mL} \mathrm{THF} / 1 \mathrm{mmol}$ Li-DBM). The solution of $\operatorname{Pd}(\mathrm{dba})_{2}$ was transferred by syringe to the Li-DBM suspension. After stirring for 5 minutes, alkenyl bromide (1.2 equiv) or alkenyl iodide (1.2 equiv) was added neat and the reaction was allowed to stir overnight at room temperature.

Upon completion, an equal volume of saturated $\mathrm{NH}_{4} \mathrm{Cl}(\mathrm{aq})$ was added. Diethyl ether was added and the layers separated. The aqueous layer was extracted three times with diethyl ether and the combined organic extracted were dried over $\mathrm{MgSO}_{4}$, filtered and concentrated in vacuo. Products were purified by silica gel flash column chromatography (20:1 -> 10:1 hexanes/ethyl acetate). 
(E)-2,2'-(3-methylhept-2-ene-1,1-diyl)bis(4,4,5,5-tetramethyl-1,3,2-dioxaborolane) (2a)

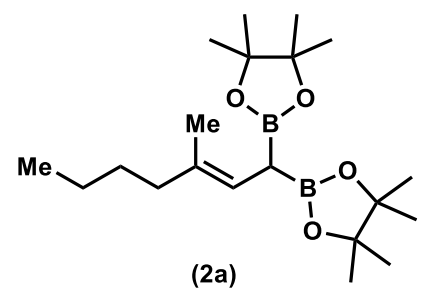

Diboronic ester (2a) was prepared according to a literature procedure ${ }^{3}$.

(E)-2,2'-(3-methylpent-2-ene-1,1-diyl)bis(4,4,5,5-tetramethyl-1,3,2-dioxaborolane) (2b)

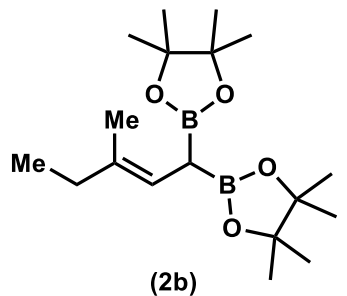

Diboronic ester (2b) was prepared according to a literature procedure ${ }^{3}$.

(Z)-2,2'-(3-methylpent-2-ene-1,1-diyl)bis(4,4,5,5-tetramethyl-1,3,2-dioxaborolane) (2c)

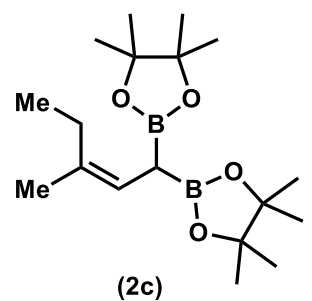

Diboronic ester (2c) was prepared according to a literature procedure ${ }^{3}$.

(E)-2,2'-(3-cyclohexylbut-2-ene-1,1-diyl)bis(4,4,5,5-tetramethyl-1,3,2-dioxaborolane) (2d)<smiles>[Y4]C(=CC(B1OC(C(C)(C)C)C(C)(C)O1)B1OC(C)(C)C(C)(C)O1)C1CCCCC1</smiles>

Diboronic ester (2d) was prepared according to a literature procedure ${ }^{3}$.

(E)-2,2'-(3-cyclopropylbut-2-ene-1,1-diyl)bis(4,4,5,5-tetramethyl-1,3,2-dioxaborolane) (2e) 


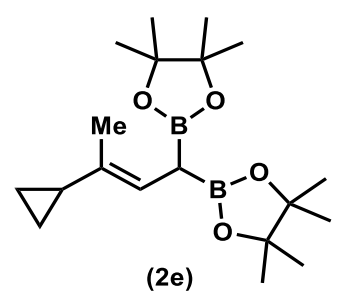

Diboronic ester (2e) was prepared according to a literature procedure ${ }^{3}$.

2,2'-(3-methylbut-2-ene-1,1-diyl)bis(4,4,5,5-tetramethyl-1,3,2-dioxaborolane) (2f)

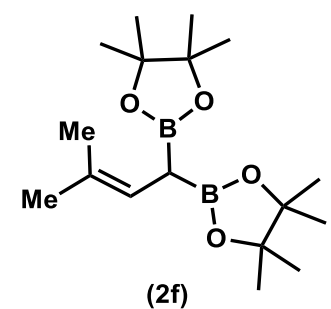

Diboronic ester (2f) was prepared according to a literature procedure ${ }^{3}$.

(E)-2,2'-(3-methyl-5-phenylpent-2-ene-1,1-diyl)bis(4,4,5,5-tetramethyl-1,3,2-dioxaborolane) (2g)

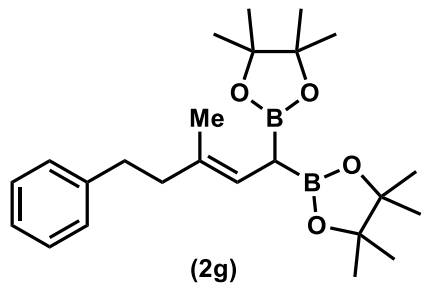

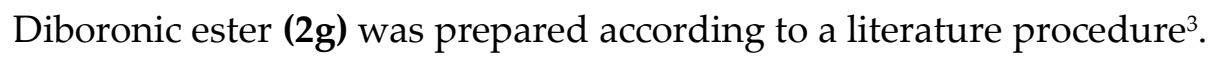

(E)-tert-butyldimethyl((3-methyl-5,5-bis(4,4,5,5-tetramethyl-1,3,2-dioxaborolan-2-yl)pent-3en-1-yl)oxy)silane (2h)

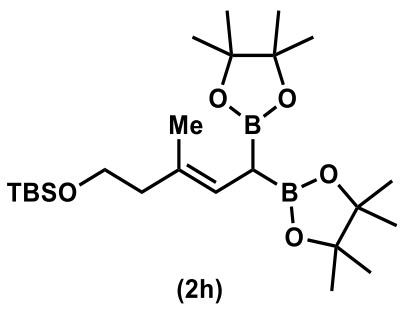

Diboronic ester (2h) was prepared according to a literature procedure 3 .

(E)-4,4,5,5-tetramethyl-2-(3-methylhept-2-en-1-yl)-1,3,2-dioxaborolane (7) 


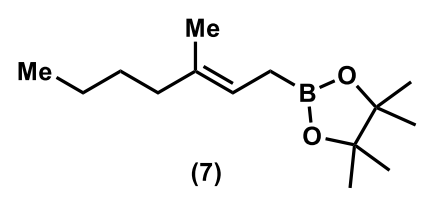

In a $\mathrm{N}_{2}$-filled glovebox, to an $8 \mathrm{~mL}$ vial charged with magnetic stirrer bar was added $\mathrm{KOtBu}$ (92.4 mg, $0.824 \mathrm{mmol}, 1.0$ equiv) and THF (5 mL). To this solution was added neat diboron reagent (2a, $300 \mathrm{mg}, 0.824 \mathrm{mmol}, 1.0$ equiv). This vial was then sealed with a septa cap and removed from the glovebox. After stirring for 15 minutes, dry iPrOH (631 $\mu \mathrm{L}, 10.0$ equiv) was added to the pink reaction mixture. The mixture was allowed to stir for $16 \mathrm{~h}$, at which point $\mathrm{Et}_{2} \mathrm{O}$ and sat. $\mathrm{NH}_{4} \mathrm{Cl}$ (aq.) $(2 \mathrm{~mL})$ were added. The layers were allowed to separate, and the aqueous layer extracted with $\mathrm{Et}_{2} \mathrm{O}(3 \times 1 \mathrm{~mL})$. The combined organic layers were dried $\left(\mathrm{MgSO}_{4}\right)$, filtered and concentrated by rotary evaporation. Flash silica gel chromatography (80:1 -> 40:1 hexanes/ethyl acetate) afforded the monoboryl reagent as a clear, colorless oil in $80 \%$ yield $(157 \mathrm{mg}, 0.659 \mathrm{mmol}) .{ }^{1} \mathbf{H}$

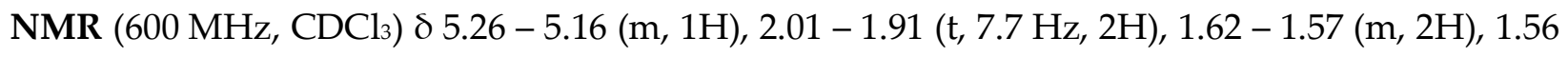
$(\mathrm{s}, 3 \mathrm{H}), 1.38-1.30(\mathrm{~m}, 2 \mathrm{H}), 1.30-1.24(\mathrm{~m}, 2 \mathrm{H}), 1.23(\mathrm{~s}, 12 \mathrm{H}), 0.91-0.83(\mathrm{t}, 7.3 \mathrm{~Hz}, 3 \mathrm{H}) .{ }^{13} \mathrm{C} \mathrm{NMR}$ $\left(151 \mathrm{MHz} \mathrm{CDCl}_{3}\right) \delta$ 135.6, 118.3, 83.2, 39.6, 30.4, 24.9, 22.4, 15.9, 14.2. HRMS (GC-HRMS, EI, m/z) calc for $\left[\mathrm{M}+\mathrm{H}^{+}\right] \mathrm{C}_{14} \mathrm{H}_{28} \mathrm{BO}_{2}{ }^{+}$: 239.2177, found: 239.2192. IR $\left(\mathrm{cm}^{-1}\right) 2687(\mathrm{~m}), 2308(\mathrm{~m}), 2055(\mathrm{~m}), 1722$ (w), $1611(\mathrm{~m}), 1367(\mathrm{~m}), 1227(\mathrm{~s}), 1147(\mathrm{~m}), 739$ (s).

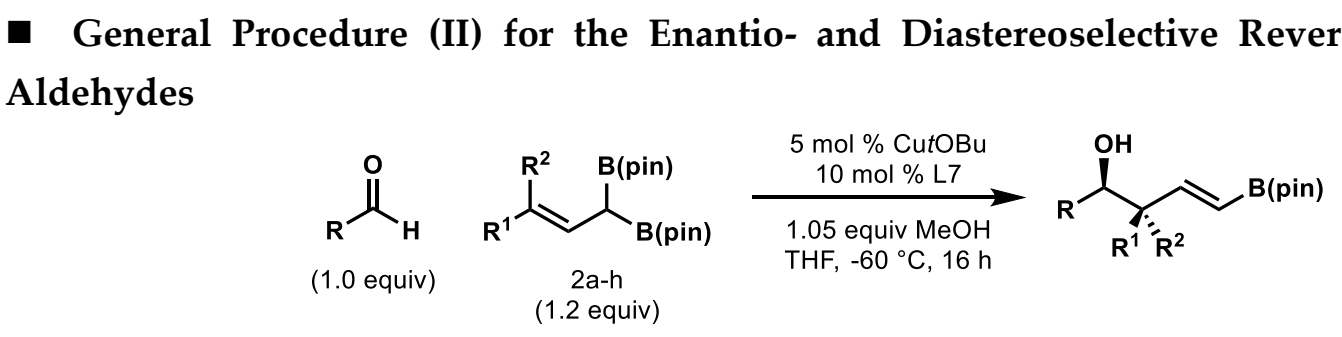

In a $\mathrm{N}_{2}$-filled glovebox, (R)-di-iPr-4-OMe-phosphoramidite (L7) $(3.7 \mathrm{mg}, 0.005 \mathrm{mmol}, 10 \mathrm{~mol}$ $\%$ ) was weighed into an $8 \mathrm{~mL}$ vial charged with magnetic stir bar. To this vial, $200 \mu \mathrm{L}$ of a CuOtBu solution (12.5 mM, $0.0025 \mathrm{mmol}, 0.05$ equiv) was added affording a pale yellow solution. In a second $8 \mathrm{~mL}$ vial, a stock solution of the 1,1 -bis-boronate $(0.60 \mathrm{M}$ in THF) was prepared. In a third $8 \mathrm{~mL}$ vial, a stock solution of the aldehyde (0.50 M in THF) was prepared. All vials were capped with a Teflon-lined lid, sealed with electrical tape, and removed from the glovebox.

All vials were cooled to $-78{ }^{\circ} \mathrm{C}$ (dry ice/acetone bath) and $100 \mu \mathrm{L}$ diboron stock solution $(0.060$ mmol, 1.2 equiv) was added dropwise to the vial containing catalyst and ligand. After 2 minutes, $50 \mu \mathrm{L}$ aldehyde stock solution $(0.050 \mathrm{mmol}, 1.0$ equiv) was added followed by room temperature, dry methanol ( $2.1 \mu \mathrm{L}, 0.0525 \mathrm{mmol}, 1.05$ equiv). The reactions were taped over with Teflon and electrical tape and allowed to sit in a $-60{ }^{\circ} \mathrm{C}$ cryobath for 12 hours.

Upon completion, the reactions were quenched at $-60{ }^{\circ} \mathrm{C}$ with excess $\mathrm{NaBH}_{4}(\sim 10$ equivalents, added as a solid) and $\mathrm{MeOH}(300 \mu \mathrm{L})$ and allowed to stir at $-60{ }^{\circ} \mathrm{C}$ for 15 minutes. Saturated, 
aqueous $\mathrm{NH}_{4} \mathrm{Cl}(1 \mathrm{~mL})$ was added and the reaction mixture was allowed to warm to room temperature and stirred for a further 30 minutes. Diethyl ether was added and the layers separated. The aqueous layer was extracted three times with diethyl ether $(3 \times 1 \mathrm{~mL})$ and the combined organic extracts were dried over $\mathrm{MgSO}_{4}$, filtered and concentrated in vacuo. NMR yields were determined by ${ }^{1} \mathrm{H}$ NMR using hexamethyldisiloxane $(5 \mu \mathrm{L})$ as an internal standard. Products were purified by flash silica gel chromatography. Subsequent analysis by HPLC or SFC provided enantiomeric ratios.

- General Procedure (III) for the Enantio- and Diastereoselective Reverse Allylation of Aldehydes

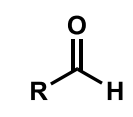

(1.0 equiv)
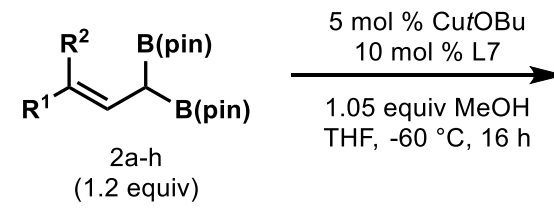

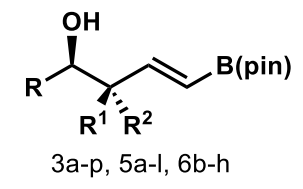

In a N2-filled glovebox, (R)-di-iPr-4-OMe-phosphoramidite (L7) (3.7 mg, $0.005 \mathrm{mmol}, 10 \mathrm{~mol}$ $\%$ ) was weighed into an $8 \mathrm{~mL}$ vial charged with magnetic stir bar. To this vial, $200 \mu \mathrm{L}$ of a CuOtBu solution (12.5 mM, $0.0025 \mathrm{mmol}, 0.05$ equiv) was added affording a pale yellow solution. In a second $8 \mathrm{~mL}$ vial, a stock solution of the 1,1-bis-boronate ( $0.60 \mathrm{M}$ in THF) was prepared. In a third $8 \mathrm{~mL}$ vial, a stock solution of the aldehyde (0.50 M in THF) was prepared. All vials were capped with a Teflon-lined lid, sealed with electrical tape, and removed from the glovebox.

All vials were cooled to $-78{ }^{\circ} \mathrm{C}$ (dry ice/acetone bath) and $100 \mu \mathrm{L}$ diboron stock solution $(0.060$ mmol, 1.2 equiv) was added dropwise to the vial containing catalyst and ligand. After 2 minutes, $50 \mu \mathrm{L}$ aldehyde stock solution ( $0.050 \mathrm{mmol}, 1.0$ equiv) was added followed by room temperature, dry methanol ( $2.1 \mu \mathrm{L}, 0.0525 \mathrm{mmol}, 1.05$ equiv). The reactions were taped over with Teflon and electrical tape and allowed to sit in a $-60^{\circ} \mathrm{C}$ cryobath for 12 hours.

Upon completion, the reactions were quenched at $-60{ }^{\circ} \mathrm{C}$ with $1 \mathrm{~mL}$ saturated $\mathrm{NH}_{4} \mathrm{Cl}$ (aq) and stirred vigorously at $22^{\circ} \mathrm{C}$ for 30 minutes. Diethyl ether was added and the layers separated. The aqueous layer was extracted three times with diethyl ether $(3 \times 1 \mathrm{~mL})$ and the combined organic extracts were dried over $\mathrm{MgSO}_{4}$, filtered and concentrated in vacuo. NMR yields were determined by ${ }^{1} \mathrm{H}$ NMR using hexamethyldisiloxane $(5 \mu \mathrm{L})$ as an internal standard. Products were purified by flash silica gel chromatography. Subsequent analysis by HPLC or SFC provided enantiomeric ratios. 
- Product Characterization

(1S,2S)-2-methyl-1-phenyl-2-((E)-2-(4,4,5,5-tetramethyl-1,3,2-dioxaborolan-2-yl)vinyl)hexan-1-ol (3a)

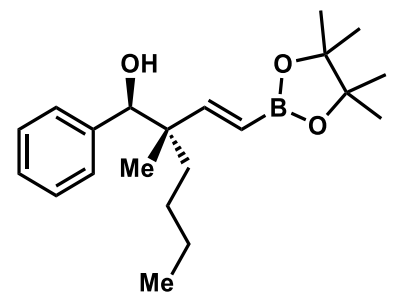

(3a)

Prepared according to General Method III. Purified by silica gel column chromatography (Hexanes/Ethyl Acetate 25:1, 20:1, 15:1, 10:1) to give the product (13.4 mg, 79\% yield, 98.5:1.5 er, 4:1 dr) as a colorless oil.

\section{Major Diastereomer}

${ }^{1} \mathrm{H}$ NMR $\left(600 \mathrm{MHz}, \mathrm{CDCl}_{3}\right) \delta 7.30$ - $7.23(\mathrm{~m}, 5 \mathrm{H}), 6.68-6.62(\mathrm{~d}, 18.5 \mathrm{~Hz}, 1 \mathrm{H}), 5.54-5.46(\mathrm{~d}, 18.5$ $\mathrm{Hz}, 1 \mathrm{H}), 4.48(\mathrm{~s}, 1 \mathrm{H}), 1.29(\mathrm{~s}, 12 \mathrm{H}), 1.24-1.16(\mathrm{~m}, 6 \mathrm{H}), 0.91(\mathrm{~s}, 3 \mathrm{H}), 0.83(\mathrm{t}, 7.2 \mathrm{~Hz}, 3 \mathrm{H}) .{ }^{13} \mathrm{C}$ NMR $\left(151 \mathrm{MHz}, \mathrm{CDCl}_{3}\right) \delta 159.5,140.5,128.2,127.6,127.6,83.4,79.9,47.5,37.4,26.4,25.0,24.9,23.6,16.1$, 14.2 .

\section{Minor Diastereomer}

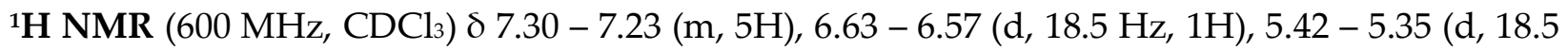
$\mathrm{Hz}), 4.49$ (s, 1H), $1.28(\mathrm{~s}, 12 \mathrm{H}), 1.14-1.08(\mathrm{~m}, 6 \mathrm{H}), 1.00(\mathrm{~s}, 3 \mathrm{H}), 0.83(\mathrm{t}, 7.2 \mathrm{~Hz}, 3 \mathrm{H}) .{ }^{13} \mathrm{C}$ NMR $(151$ $\left.\mathrm{MHz}_{2} \mathrm{CDCl}_{3}\right) \delta 158.6,141.1,127.9,127.5,127.5,83.3,80.8,46.8,35.4,26.5,25.0,24.8,23.7,19.0,14.3$. HRMS (m/z) calc. for [M- $\left.\mathrm{H}_{2} \mathrm{O}^{+}\right] \mathrm{C}_{21} \mathrm{H}_{32} \mathrm{BO}_{2}{ }^{+}: 327.2490$ found: 327.2490. IR ( $\left.\mathrm{cm}^{-1}\right) 3481(\mathrm{w}), 2931(\mathrm{~m})$, $2861(\mathrm{w}), 1633(\mathrm{~m}), 1456(\mathrm{w}), 1351$ (s), $1146(\mathrm{~s}), 970(\mathrm{w}), 703(\mathrm{w}) .[\alpha]_{\mathrm{D}}^{23}=-30.2$ (c = 0.67, $\mathrm{CH}_{2} \mathrm{Cl}_{2}, 1$ $=1 \mathrm{dm})$.

Phenomenex lux Amylose 1-AD Column: 97:3 $\mathrm{CO}_{2}: \mathrm{iPrOH} ; 1.0 \mathrm{~mL} / \mathrm{min} ; 210 \mathrm{~nm}$.

\section{Racemic material:}

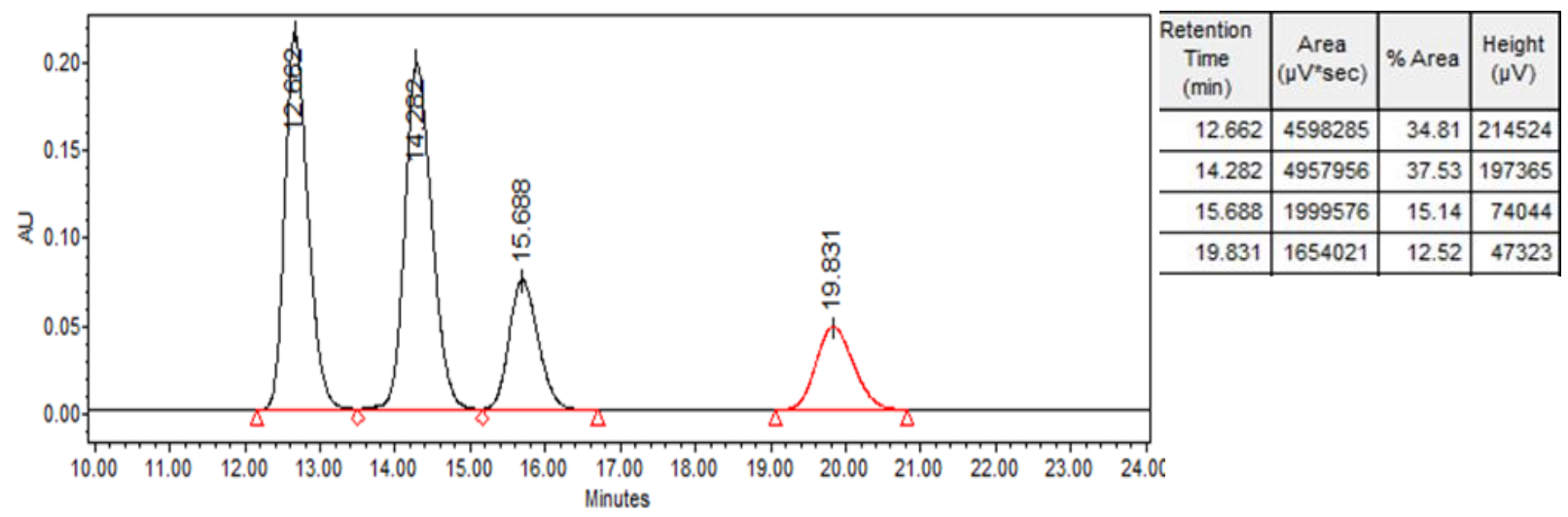

Enantioenriched material:

Major: 98.5:1.5 er 


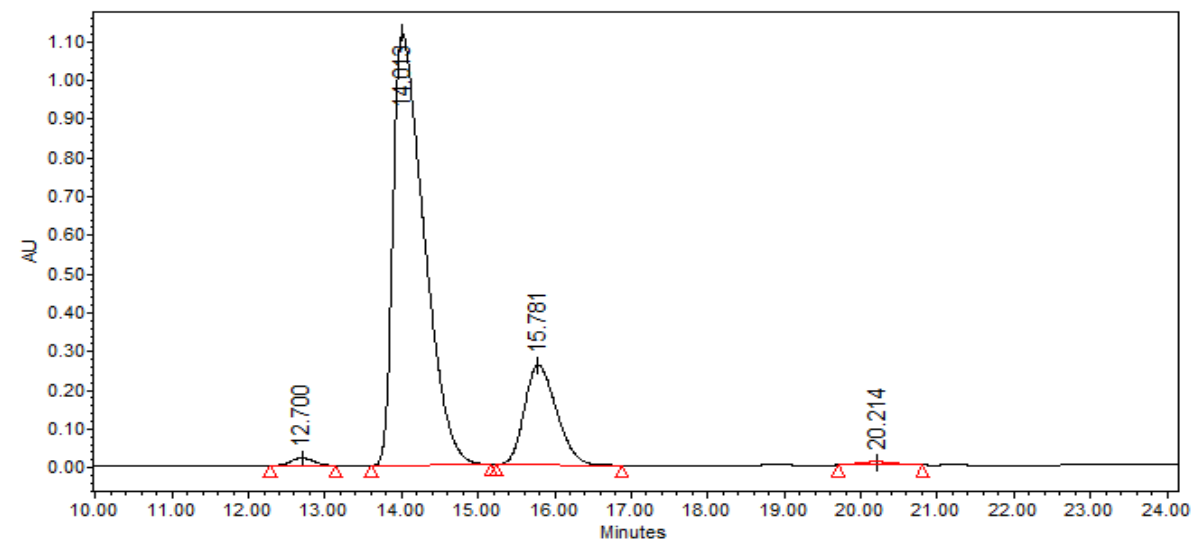

\begin{tabular}{c|r|r|r|}
\hline $\begin{array}{c}\text { Retention } \\
\text { Time } \\
(\mathrm{min})\end{array}$ & $\begin{array}{c}\text { Area } \\
\left(\mu \mathrm{V}^{*} \mathrm{sec}\right)\end{array}$ & $\%$ Area & $\begin{array}{c}\text { Height } \\
(\mu \mathrm{V})\end{array}$ \\
\hline 12.700 & 421792 & 1.06 & 20369 \\
\hline 14.013 & 31411849 & 79.29 & 1114465 \\
\hline 15.781 & 7504527 & 18.94 & 257634 \\
\hline 20.214 & 276148 & 0.70 & 8510 \\
\hline
\end{tabular}

(1S,2S)-1-(4-methoxyphenyl)-2-methyl-2-((E)-2-(4,4,5,5-tetramethyl-1,3,2-dioxaborolan-2-yl)vinyl)hexan1 -ol (3b)

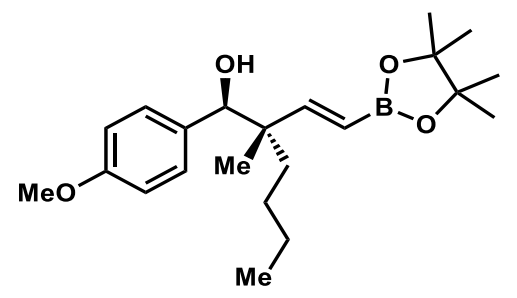

(3b)

Prepared according to General Method III. Purified by silica gel column chromatography (Hexanes/Ethyl Acetate 25:1, 20:1, 15:1, 10:1) to give the product (14.3 mg, 76\% yield, 97.5:2.5 er, 3:1 dr) as a colorless oil.

\section{Major Diastereomer}

${ }^{1} \mathrm{H}$ NMR $\left(600 \mathrm{MHz}, \mathrm{CDCl}_{3}\right) \delta 7.23-7.19(\mathrm{~d}, 8.6 \mathrm{~Hz}, 2 \mathrm{H}), 6.86-6.81(\mathrm{~d}, 8.6 \mathrm{~Hz}, 2 \mathrm{H}), 6.68$ - $6.62(\mathrm{~d}$, $18.5 \mathrm{~Hz}, 1 \mathrm{H}), 5.53-5.46(\mathrm{~d}, 18.5 \mathrm{~Hz}, 1 \mathrm{H}), 4.43(\mathrm{~s}, 1 \mathrm{H}), 3.80(\mathrm{~s}, 3 \mathrm{H}), 1.29(\mathrm{~s}, 12 \mathrm{H}), 1.23$ - $1.06(\mathrm{~m}$, $6 \mathrm{H}), 0.89$ (s, 3H), $0.86-0.80$ (t, $7.3 \mathrm{~Hz}, 3 \mathrm{H}) .{ }^{13} \mathrm{C}$ NMR $\left(151 \mathrm{MHz}, \mathrm{CDCl}_{3}\right) \delta$ 159.6, 159.0, 132.6, 129.2, 113.0, 83.4, 79.6, 55.4, 47.7, 37.5, 26.4, 25.0, 25.0, 23.6, 16.1, 14.3.

\section{Minor Diastereomer}

${ }^{1} \mathrm{H}$ NMR $\left(600 \mathrm{MHz}, \mathrm{CDCl}_{3}\right) \delta 7.19-7.15(\mathrm{~d}, 8.6 \mathrm{~Hz}, 2 \mathrm{H}), 6.82-6.80(\mathrm{~d}, 8.7 \mathrm{~Hz}, 2 \mathrm{H}), 6.62-6.57(\mathrm{~d}$, $18.6 \mathrm{~Hz}, 1 \mathrm{H}), 5.40-5.37(\mathrm{~d}, 18.5 \mathrm{~Hz}, 1 \mathrm{H}), 4.44(\mathrm{~s}, 1 \mathrm{H}), 3.80(\mathrm{~s}, 3 \mathrm{H}), 1.28(\mathrm{~s}, 12 \mathrm{H}), 1.23$ - $1.06(\mathrm{~m}$, $6 \mathrm{H}), 0.98(\mathrm{~s}, 3 \mathrm{H}), 0.86-0.80(\mathrm{t}, 7.3 \mathrm{~Hz}, 3 \mathrm{H}) .{ }^{13} \mathrm{C} \mathbf{N M R}\left(151 \mathrm{MHz}, \mathrm{CDCl}_{3}\right) \delta 158.9,158.8,133.3,129.0$, $112.9,83.3,80.4,46.9,35.3,29.9,26.5,25.0,24.8,23.7,19.0,14.3$.

HRMS (m/z) calc. for [M- $\left.\mathrm{H}_{2} \mathrm{O}^{+}\right] \mathrm{C}_{22} \mathrm{H}_{34} \mathrm{BO}_{3}{ }^{+}: 357.2596$ found: 357.2594. IR (cm $\left.{ }^{-1}\right) 2930(\mathrm{~m}), 2859(\mathrm{w})$, $1634(\mathrm{~m}), 1508(\mathrm{~m}), 1350(\mathrm{~s}), 1248(\mathrm{~m}), 1146(\mathrm{~m}), 970(\mathrm{w}), 851(\mathrm{w}) .[\alpha]^{23}=-13.0\left(\mathrm{c}=0.72, \mathrm{CH}_{2} \mathrm{Cl}_{2}\right.$, $1=1 \mathrm{dm})$.

Diacel CHIRALPAK IE Column: 94:6 hexanes: $\mathrm{PrOH} ; 0.4 \mathrm{~mL} / \mathrm{min} ; 210 \mathrm{~nm}$. Racemic material: 


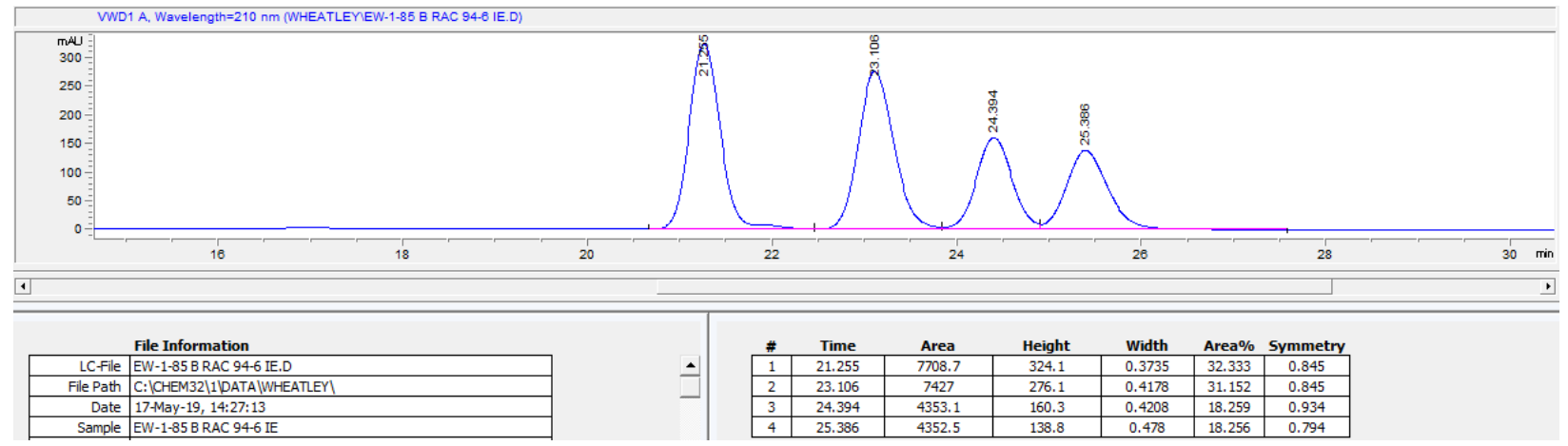

\section{Enantioenriched material:}

Major: 97.5:2.5 er

Minor: $97: 3$ er

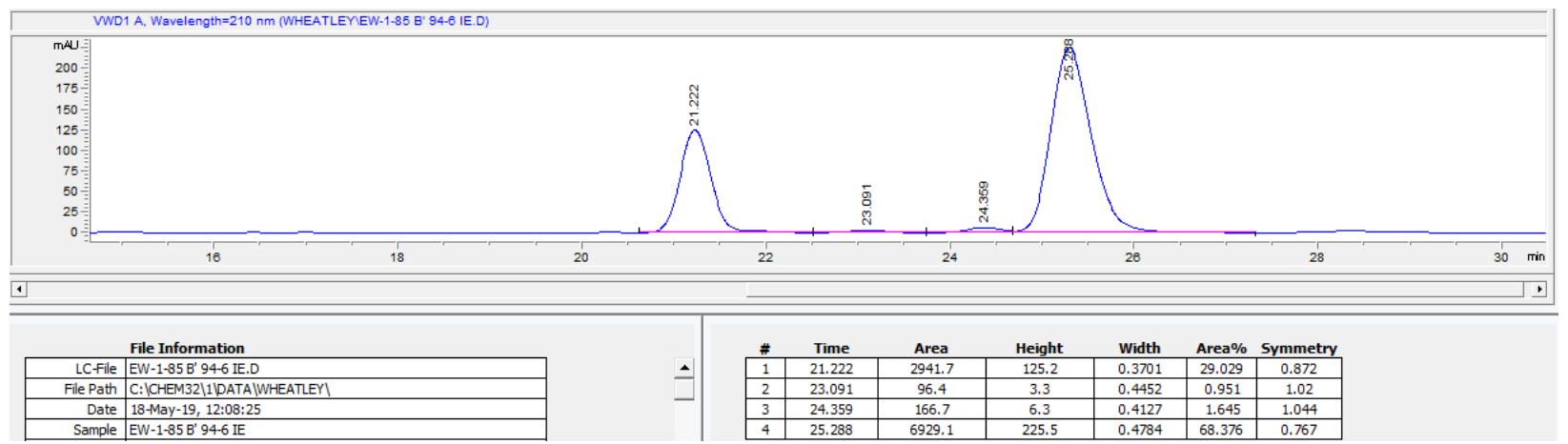

(1S,2S)-1-(4-fluorophenyl)-2-methyl-2-((E)-2-(4,4,5,5-tetramethyl-1,3,2-dioxaborolan-2-yl)vinyl)hexan-1-ol (3c)<smiles>CCCC[C@](C)(/C=C/B1OC(C)(C)C(C)(C)O1)[C@@H](O)c1ccc(F)cc1</smiles>

(3c)

Prepared according to General Method III. Purified by silica gel column chromatography (Hexanes/Ethyl Acetate 25:1, 20:1, 15:1, 10:1) to give the product (16.4 mg, 91\% yield, 98.5:1.5 er, 7:1 dr) as a colorless oil (yield was adjusted to take protodeboration impurity into account). ${ }^{1} \mathbf{H}$ NMR $\left(600 \mathrm{MHz}, \mathrm{CDCl}_{3}\right) \delta 7.28-7.24(\mathrm{~m}, 2 \mathrm{H}), 7.01-6.96(\mathrm{~m}, 2 \mathrm{H}), 6.65-6.59$ (d, $\left.18.4 \mathrm{~Hz}, 1 \mathrm{H}\right), 5.54$ - $5.47(\mathrm{~d}, 18.4 \mathrm{~Hz}, 1 \mathrm{H}), 4.46(\mathrm{~s}, 1 \mathrm{H}), 1.94(\mathrm{~s}, 1 \mathrm{H}), 1.29(\mathrm{~s}, 12 \mathrm{H}), 1.24-1.18(\mathrm{~m}, 3 \mathrm{H}), 1.16$ - 1.07 (m, $3 \mathrm{H}), 0.89(\mathrm{~s}, 3 \mathrm{H}), 0.86-0.81(\mathrm{t}, 7.3 \mathrm{~Hz}, 3 \mathrm{H}) .{ }^{13} \mathrm{C}$ NMR $\left(151 \mathrm{MHz}, \mathrm{CDCl}_{3}\right) \delta 163.1-161.5(\mathrm{~d}, 242 \mathrm{~Hz})$, 159.1, 136.1 - 136.1 (d, $2.4 \mathrm{~Hz}), 129.7$ - 129.7 (d, 8.2 Hz), 114.5 - 114.4 (d, $20.8 \mathrm{~Hz}), 83.4$, 79.3, 47.5, 
37.4, 26.4, 25.0, 24.9, 23.6, 16.0, 14.2. ${ }^{19} \mathrm{~F}$ NMR (376 MHz, $\left.\mathrm{CDCl}_{3}\right) \delta$-115.4. HRMS (m/z) calc. for [M-H $\left.\mathrm{O}^{+}\right] \mathrm{C}_{21} \mathrm{H}_{31} \mathrm{BFO}_{2}{ }^{+}: 345.2396$ found: 345.2392. IR (cm-1) 2931 (m), 2860 (w), 1635 (m), 1508 (s), $1351(\mathrm{~s}), 1224(\mathrm{~m}), 1146(\mathrm{~m}), 851(\mathrm{~m}) .[\alpha]_{\mathrm{D}^{23}}=-18.3\left(\mathrm{c}=0.86, \mathrm{CH}_{2} \mathrm{Cl}_{2}, \mathrm{l}=1 \mathrm{dm}\right)$.

Phenomenex lux Cellulose 1-OD Column: 95:5 $\mathrm{CO}_{2}: \mathrm{iPrOH} ; 1.0 \mathrm{~mL} / \mathrm{min} ; 210 \mathrm{~nm}$.

Racemic material:

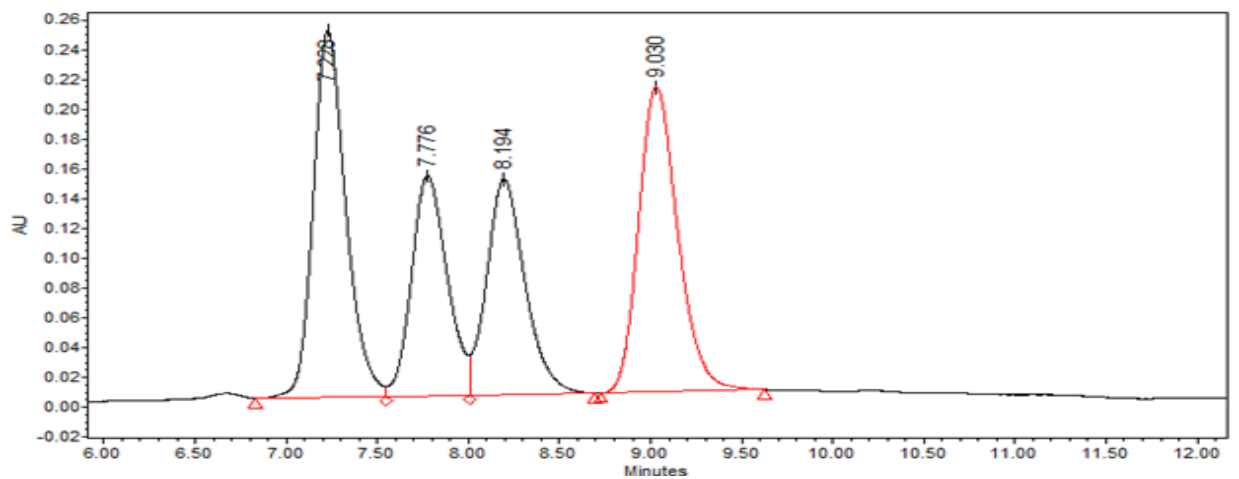

\begin{tabular}{c|c|c|c|}
\hline $\begin{array}{c}\text { Retention } \\
\text { Time } \\
(\mathrm{min})\end{array}$ & $\begin{array}{c}\text { Area } \\
\left(\mu \mathrm{V}^{\prime} \mathrm{sec}\right)\end{array}$ & \% Area & $\begin{array}{c}\text { Height } \\
(\mu \mathrm{V})\end{array}$ \\
\hline 7.228 & 3055032 & 29.82 & 245951 \\
\hline 7.776 & 2013309 & 19.65 & 147810 \\
\hline 8.194 & 2119557 & 20.69 & 144778 \\
\hline 9.030 & 3057459 & 29.84 & 204724 \\
\hline
\end{tabular}

Enantioenriched material: 98.5:1.5 er

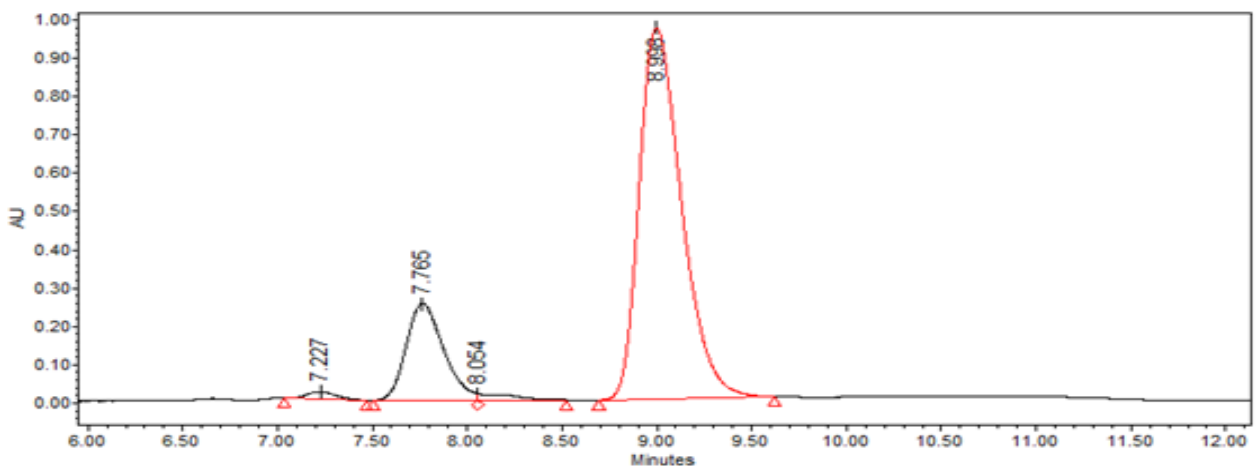

\begin{tabular}{r|r|r|r|}
\hline $\begin{array}{c}\text { Retention } \\
\text { Time } \\
(\mathrm{min})\end{array}$ & $\begin{array}{c}\text { Area } \\
(\mu \mathrm{V} \text { "sec) }\end{array}$ & \%Area & $\begin{array}{c}\text { Height } \\
(\mu \mathrm{V})\end{array}$ \\
\hline 7.227 & 195422 & 1.04 & 18616 \\
\hline 7.765 & 3435966 & 18.23 & 251880 \\
\hline 8.054 & 211384 & 1.12 & 16465 \\
\hline 8.998 & 15003347 & 79.61 & 968066 \\
\hline
\end{tabular}

(1S,2S)-1-(4-bromophenyl)-2-methyl-2-((E)-2-(4,4,5,5-tetramethyl-1,3,2-dioxaborolan-2-yl)vinyl)hexan-1ol (3d)

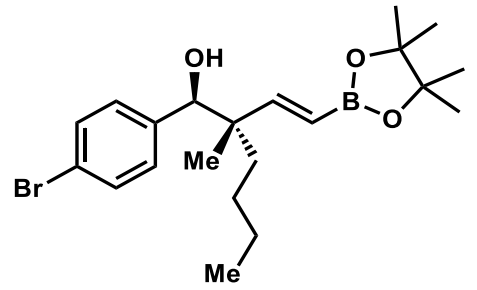

(3d)

Prepared according to General Method III. Purified by silica gel column chromatography (Hexanes/Ethyl Acetate 25:1, 20:1, 15:1, 10:1) to give the product (15.1 mg, 71\% yield, 98.5:1.5 er, 10:1 dr) as a colorless oil. ${ }^{1} \mathrm{H}$ NMR $\left(600 \mathrm{MHz}, \mathrm{CDCl}_{3}\right) \delta 7.44-7.40(\mathrm{~d}, 8.4 \mathrm{~Hz}, 2 \mathrm{H}), 7.18-7.14(\mathrm{~d}$, $8.4 \mathrm{~Hz}, 2 \mathrm{H}), 6.63-6.56(\mathrm{~d}, 18.5 \mathrm{~Hz}, 1 \mathrm{H}), 5.54-5.46(\mathrm{~d}, 18.5 \mathrm{~Hz}, 1 \mathrm{H}), 4.43$ (s, 1H), $1.82(\mathrm{~s}, 1 \mathrm{H}), 1.29$ $(\mathrm{s}, 12 \mathrm{H}), 1.22-1.18(\mathrm{~m}, 3 \mathrm{H}), 1.13-1.07(\mathrm{~m}, 3 \mathrm{H}), 0.88(\mathrm{~s}, 3 \mathrm{H}), 0.85-0.81(\mathrm{t}, 7.3 \mathrm{~Hz}, 3 \mathrm{H}) .{ }^{13} \mathrm{C}$ NMR $\left(151 \mathrm{MHz}, \mathrm{CDCl}_{3}\right) \delta$ 158.9, 139.4, 130.7, 129.9, 121.5, 83.5, 79.3, 47.5, 37.3, 26.4, 25.0, 24.9, 23.6, 16.1, 
14.2. HRMS (m/z) calc. for $\left[\mathrm{M}-\mathrm{H}_{2} \mathrm{O}^{+}\right] \mathrm{C}_{21} \mathrm{H}_{31} \mathrm{BBrO}_{2}^{+}$: 405.1595 found: 405.1594. IR $\left(\mathrm{cm}^{-1}\right) 2931(\mathrm{~m})$, $2860(\mathrm{w}), 1635$ (m), $1373(\mathrm{~m}), 1351(\mathrm{~s}), 1145(\mathrm{~m}), 1010(\mathrm{~m}), 851(\mathrm{w}) .[\alpha]_{\mathrm{D}^{23}}=-25.5$ (c = 0.76, $\mathrm{CH}_{2} \mathrm{Cl}_{2}$, $1=1 \mathrm{dm})$.

Phenomenex lux Amylose 1-AD Column: 97:3 $\mathrm{CO}_{2}: \mathrm{iPrOH} ; 1.0 \mathrm{~mL} / \mathrm{min} ; 210 \mathrm{~nm}$.

Racemic material:

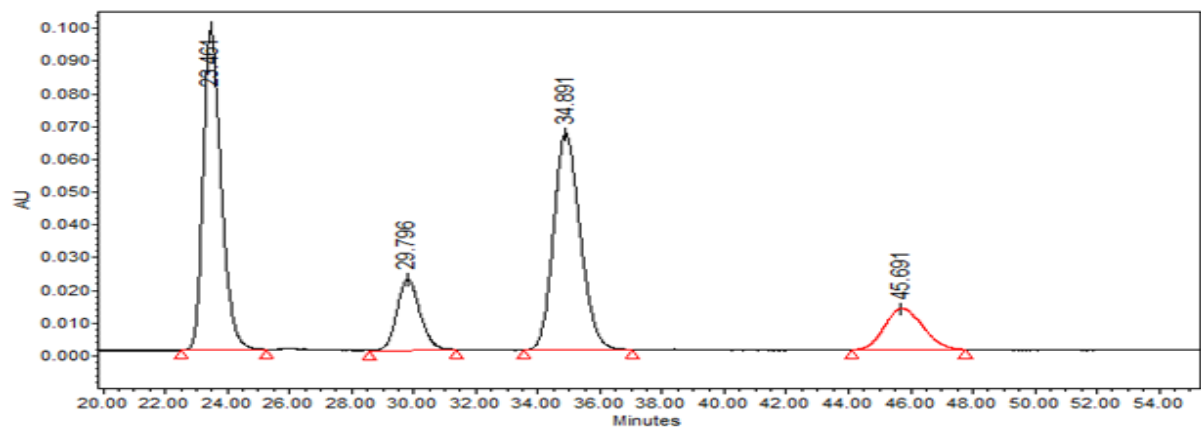

\begin{tabular}{c|c|c|c|}
\hline $\begin{array}{c}\text { Retention } \\
\text { Time } \\
(\mathrm{min})\end{array}$ & $\begin{array}{c}\text { Area } \\
\left(\mu \mathrm{V}^{\prime} \text { sec }\right)\end{array}$ & \% Area & $\begin{array}{c}\text { Height } \\
(\mu \mathrm{V})\end{array}$ \\
\hline 23.461 & 3940643 & 39.10 & 97882 \\
\hline 29.796 & 1108097 & 10.99 & 21809 \\
\hline 34.891 & 3949438 & 39.19 & 66102 \\
\hline 45.691 & 1080123 & 10.72 & 12601 \\
\hline
\end{tabular}

Enantioenriched material: 98.5:1.5 er

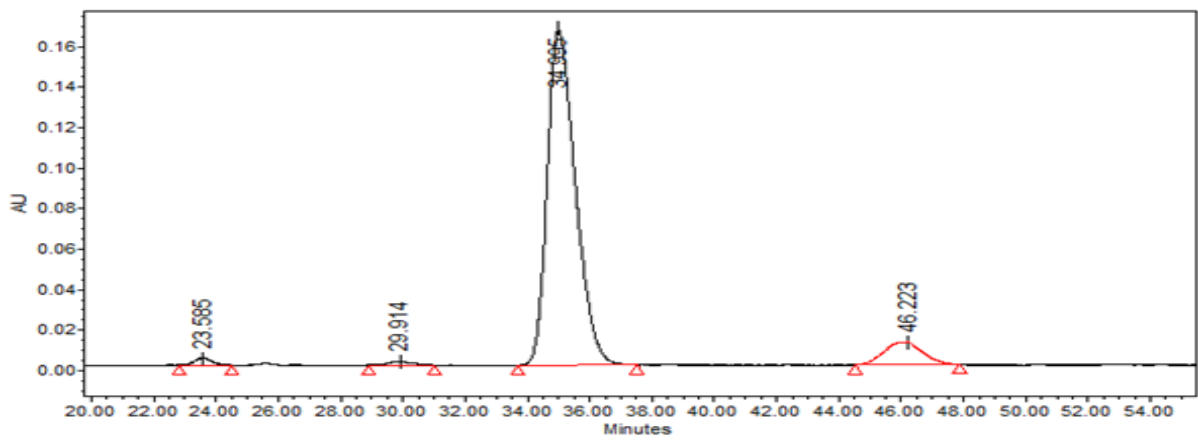

\begin{tabular}{c|r|r|r|}
\hline $\begin{array}{c}\text { Retention } \\
\text { Time } \\
(\mathrm{min})\end{array}$ & $\begin{array}{c}\text { Area } \\
\left(\mu \mathrm{V}^{*} \mathrm{sec}\right)\end{array}$ & \% Area & $\begin{array}{c}\text { Height } \\
(\mu \mathrm{V})\end{array}$ \\
\hline 23.585 & 141195 & 1.21 & 3586 \\
\hline 29.914 & 91987 & 0.79 & 1875 \\
\hline 34.995 & 10447401 & 89.83 & 166228 \\
\hline 46.223 & 949100 & 8.16 & 11256 \\
\hline
\end{tabular}

Methyl-4-((1S,2S)-1-hydroxy-2-methyl-2-((E)-2-(4,4,5,5-tetramethyl-1,3,2-dioxaborolan-2yl)vinyl)hexyl)benzoate (3e)

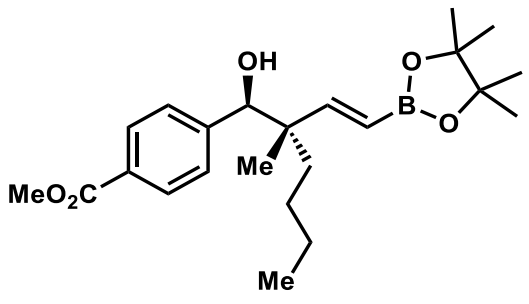

(3e)

Prepared according to General Method III. Purified by silica gel column chromatography (Hexanes/Ethyl Acetate 25:1, 20:1, 15:1, 10:1, 8:1, 5:1) to give the product (18.3 mg, 91\% yield, >99:1 er, 13:1 dr) as a colorless oil. ${ }^{1} \mathbf{H}$ NMR $\left(600 \mathrm{MHz}, \mathrm{CDCl}_{3}\right) \delta 8.00-7.94(\mathrm{~d}, 8.2 \mathrm{~Hz}, 2 \mathrm{H}), 7.40-7.30$ $(\mathrm{d}, 8.3 \mathrm{~Hz}, 2 \mathrm{H}), 6.66-6.58(\mathrm{~d}, 18.5 \mathrm{~Hz}, 1 \mathrm{H}), 5.55-5.46(\mathrm{~d}, 18.4 \mathrm{~Hz}, 1 \mathrm{H}), 4.52(\mathrm{~s}, 1 \mathrm{H}), 3.91(\mathrm{~s}, 3 \mathrm{H})$, $1.29(\mathrm{~s}, 12 \mathrm{H}), 1.24-1.07(\mathrm{~m}, 6 \mathrm{H}), 0.89(\mathrm{~s}, 3 \mathrm{H}), 0.85-0.81(\mathrm{t}, 7.3 \mathrm{~Hz}, 3 \mathrm{H}) .{ }^{13} \mathrm{C}$ NMR $(151 \mathrm{MHz}$, $\left.\mathrm{CDCl}_{3}\right) \delta 167.2,158.7,145.7,129.4,128.9,128.2,83.5,79.5,52.2,47.6,37.3,26.4,25.0,24.9,23.5,16.2$, 14.2. HRMS (m/z) calc. for $\left[\mathrm{M}-\mathrm{H}_{2} \mathrm{O}^{+}\right] \mathrm{C}_{23} \mathrm{H}_{34} \mathrm{BO}_{4}{ }^{+}: 385.2545$ found: 385.2543 . IR $\left(\mathrm{cm}^{-1}\right) 3481(\mathrm{w})$, 
2961 (s), 2932 (s), 2872 (m), 1725 (s), 1633 (m), 1436 (m), 1351 (s, 1278.6 (s), 1146 (s), 970 (w), 851 $(\mathrm{w}) .[\alpha]_{\mathrm{D}^{23}}=-19.2\left(\mathrm{c}=0.92, \mathrm{CH}_{2} \mathrm{Cl}_{2}, \mathrm{l}=1 \mathrm{dm}\right)$.

Phenomenex lux Amylose 1-AD Column: $94: 6 \mathrm{CO}_{2}: \mathrm{MeOH} ; 1.0 \mathrm{~mL} / \mathrm{min} ; 210 \mathrm{~nm}$.

Racemic material:
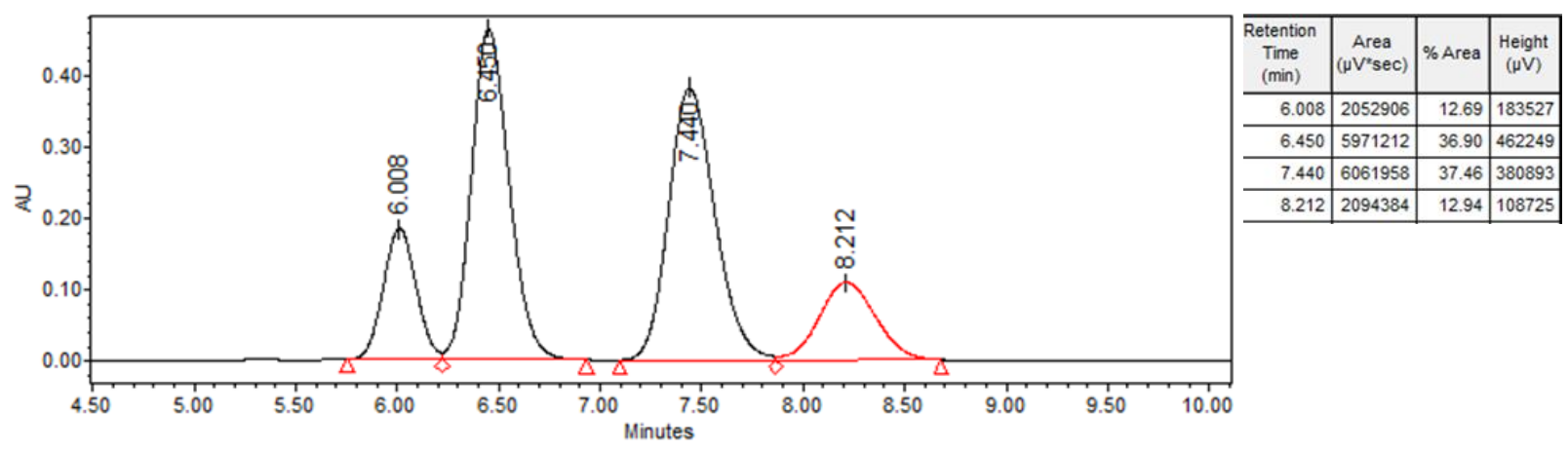

Enantioenriched material: >99:1 er
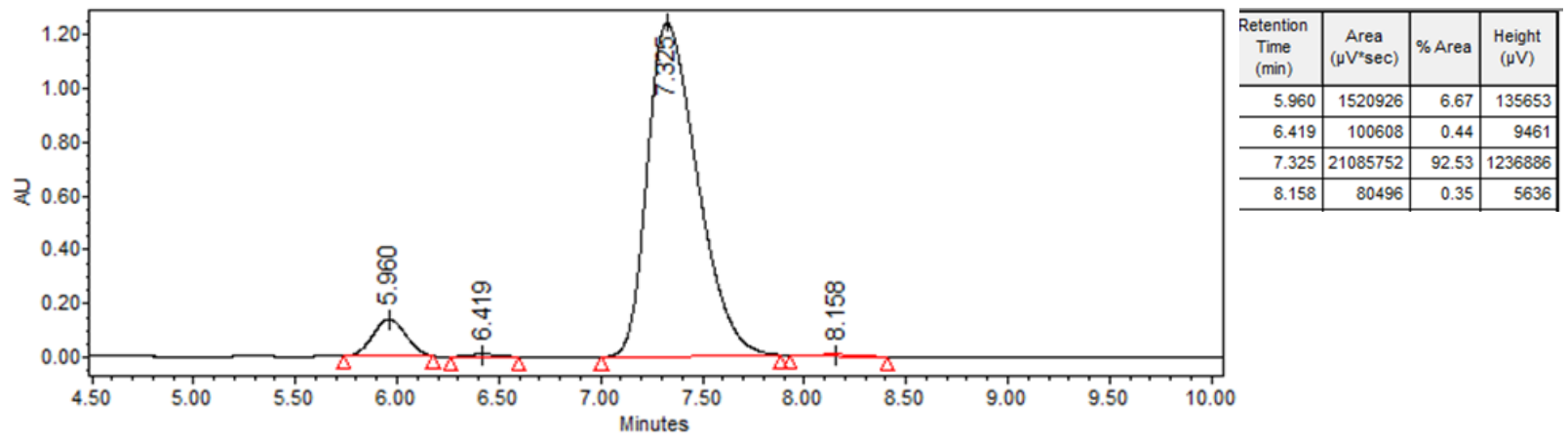

4-((1S,2S)-1-hydroxy-2-methyl-2-((E)-2-(4,4,5,5-tetramethyl-1,3,2-dioxaborolan-2yl)vinyl)hexyl)benzonitrile (3f)<smiles>CCCC[C@](C)(/C=C/B1OC(C)(C)C(C)(C)O1)[C@H](O)c1ccc(C#N)cc1</smiles>

(3f)

Prepared according to General Method III. Purified by silica gel column chromatography (Hexanes/Ethyl Acetate 25:1, 20:1, 15:1, 10:1, 8:1, 5:1) to give the product (16.5 $\mathrm{mg}$, 89\% yield, 97.5:2.5 er, 10:1 dr) as a colorless oil. ${ }^{1} \mathbf{H}$ NMR $\left(600 \mathrm{MHz}, \mathrm{CDCl}_{3}\right) \delta 7.61-7.56(\mathrm{~d}, 8.3 \mathrm{~Hz}, 1 \mathrm{H}), 7.42$ - 7.38 (d, 8.2 Hz, 2H), $6.62-6.55$ (d, $18.4 \mathrm{~Hz}, 1 \mathrm{H}), 5.54-5.45$ (d, $18.5 \mathrm{~Hz}, 1 \mathrm{H}), 4.50$ (s, 1H), 1.29 (s, 12H), 1.23 - $1.05(\mathrm{~m}, 6 \mathrm{H}), 0.88(\mathrm{~s}, 3 \mathrm{H}), 0.87-0.81(\mathrm{t}, 7.4 \mathrm{~Hz}, 3 \mathrm{H}) .{ }^{13} \mathrm{C}$ NMR $\left(151 \mathrm{MHz}, \mathrm{CDCl}_{3}\right) \delta$ $158.1,145.9,131.4,128.9,119.1,111.3,83.5,79.3,47.6,37.2,26.4,25.0,24.9,23.5,16.2$, 14.2. HRMS (m/z) calc. for $\left[\mathrm{M}-\mathrm{H}_{2} \mathrm{O}^{+}\right] \mathrm{C}_{22} \mathrm{H}_{31} \mathrm{BNO}_{2}{ }^{+}: 352.2442$ found: 352.2440. IR $\left(\mathrm{cm}^{-1}\right) 3481(\mathrm{w}), 2976(\mathrm{~m}), 2931$ 
(m), $2861(\mathrm{w}), 2228(\mathrm{~m}), 1634(\mathrm{~m}), 1380(\mathrm{~m}), 1351(\mathrm{~s}), 1321(\mathrm{~m}), 1166(\mathrm{~s}), 970(\mathrm{w}), 851(\mathrm{w}) .[\alpha]_{\mathrm{D}^{23}}-$ $17.0\left(\mathrm{c}=0.83, \mathrm{CH}_{2} \mathrm{Cl}_{2}, 1=1 \mathrm{dm}\right)$.

Phenomenex lux Cellulose 1-OD Column: $95: 5 \mathrm{CO}_{2}: \mathrm{iPrOH} ; 1.0 \mathrm{~mL} / \mathrm{min} ; 210 \mathrm{~nm}$.

Racemic material:
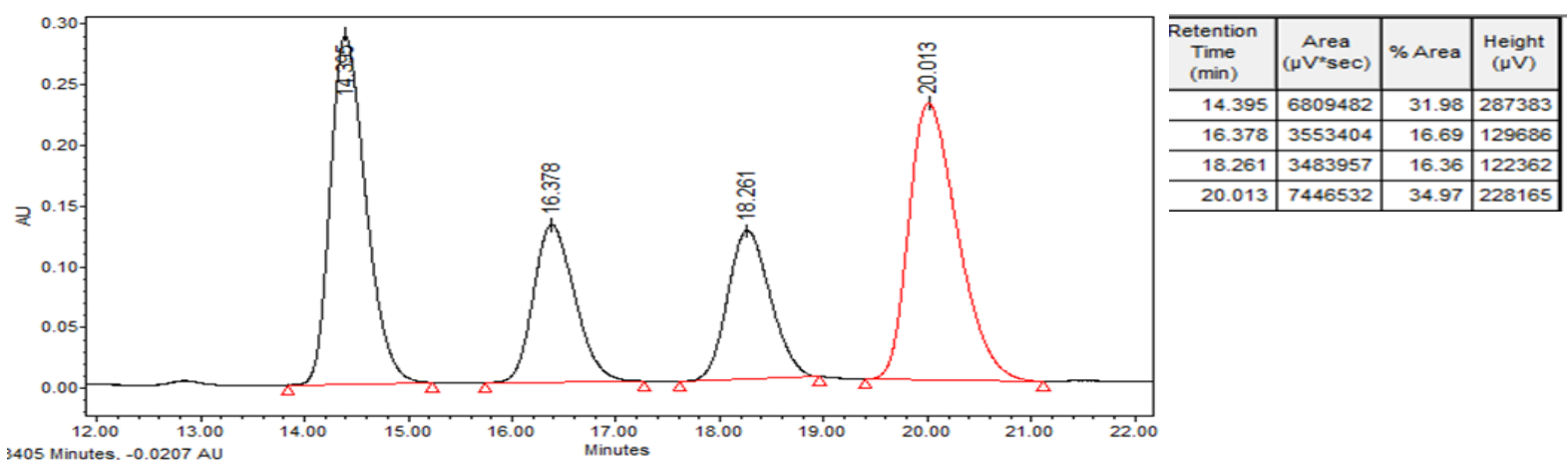

Enantioenriched material: 97.5:2.5 er

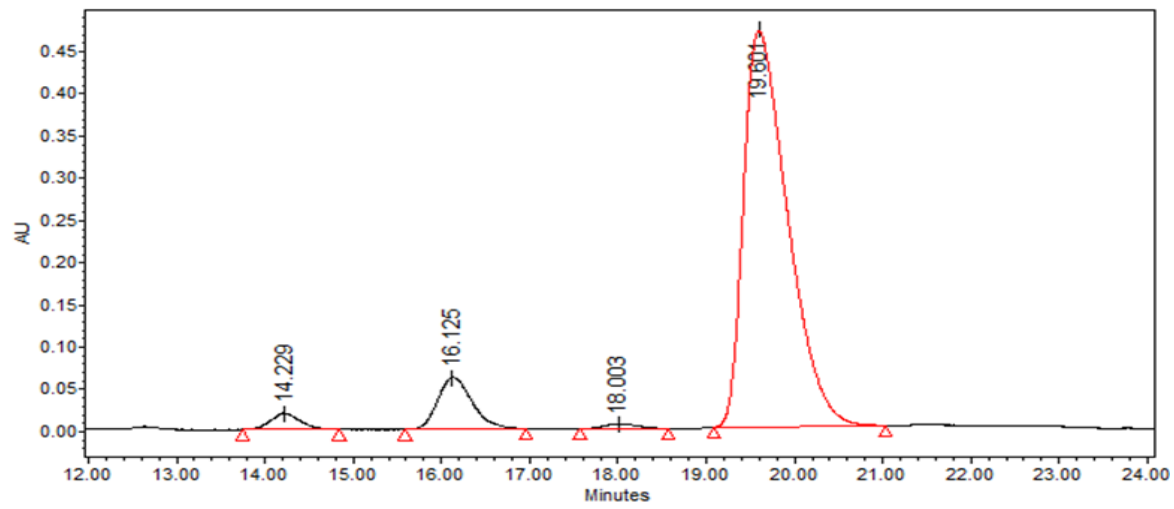

\begin{tabular}{r|r|r|r|}
\hline $\begin{array}{c}\text { Retention } \\
\text { Time } \\
(\mathrm{min})\end{array}$ & $\begin{array}{c}\text { Area } \\
(\mu \mathrm{V} \text { 'sec })\end{array}$ & \% Area & $\begin{array}{c}\text { Height } \\
(\mu \mathrm{V})\end{array}$ \\
\hline 14.229 & 439961 & 2.37 & 19266 \\
\hline 16.125 & 1643323 & 8.87 & 61067 \\
\hline 18.003 & 151711 & 0.82 & 5550 \\
\hline 19.601 & 16294832 & 87.94 & 469282 \\
\hline
\end{tabular}

(1S,2S)-2-methyl-1-(4-nitrophenyl)-2-((E)-2-(4,4,5,5-tetramethyl-1,3,2-dioxaborolan-2-yl)vinyl)hexan-1-ol (3g)

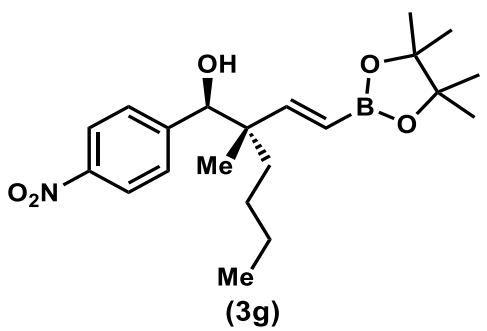

Prepared according to General Method III. Purified by silica gel column chromatography (Hexanes/Ethyl Acetate 25:1, 20:1, 15:1, 10:1, 8:1, 5:1) to give the product (16.8 $\mathrm{mg}$, 84\% yield, 98.5:1.5 er, 7:1 dr) as a colorless oil. ${ }^{1} \mathbf{H}$ NMR $\left(600 \mathrm{MHz}, \mathrm{CDCl}_{3}\right) \delta 8.20-8.13(\mathrm{~d}, 8.4 \mathrm{~Hz}, 2 \mathrm{H}), 7.50$ - $7.44(\mathrm{~d}, 8.4 \mathrm{~Hz}, 2 \mathrm{H}), 6.64-6.55(\mathrm{~d}, 18.4 \mathrm{~Hz}, 1 \mathrm{H}), 5.55-5.46(\mathrm{~d}, 18.4 \mathrm{~Hz}, 1 \mathrm{H}), 4.56(\mathrm{~s}, 1 \mathrm{H}), 2.14$ (s, $1 \mathrm{H}), 1.47-1.36(\mathrm{~m}, 2 \mathrm{H}), 1.29(\mathrm{~s}, 12 \mathrm{H}), 1.23-1.16(\mathrm{~m}, 2 \mathrm{H}), 1.14-1.07(\mathrm{~m}, 2 \mathrm{H}), 0.89(\mathrm{~s}, 3 \mathrm{H}), 0.84(\mathrm{t}$, $7.4 \mathrm{~Hz}, 3 \mathrm{H}) .{ }^{13} \mathrm{C}$ NMR $\left(151 \mathrm{MHz}, \mathrm{CDCl}_{3}\right) \delta$ 158.0, 148.0, 147.4, 129.0, 122.8, 83.6, 79.1, 47.6, 37.2, 26.4, 25.0, 24.9, 23.5, 16.2, 14.2. HRMS (m/z) calc. for $\left[\mathrm{M}-\mathrm{H}_{2} \mathrm{O}^{+}\right] \mathrm{C}_{21} \mathrm{H}_{31} \mathrm{BNO}_{4}{ }^{+}$: 372.2341 found: 
372.2340. IR (cm-1) 3458 (w), 2976 (m), 2931 (m), 2861 (w), 2360 (w), 1633 (m), 1524 (s), 1348 (s), $1146(\mathrm{~s}), 1013(\mathrm{~m}), 860(\mathrm{w}) .[\alpha]_{\mathrm{D}^{23}}=-17.5\left(\mathrm{c}=0.84, \mathrm{CH}_{2} \mathrm{Cl}_{2}, 1=1 \mathrm{dm}\right)$.

Diacel CHIRALPAK IC Column: 97:3 $\mathrm{CO}_{2}: \mathrm{iPrOH} ; 1.0 \mathrm{~mL} / \mathrm{min} ; 210 \mathrm{~nm}$.

Racemic material:

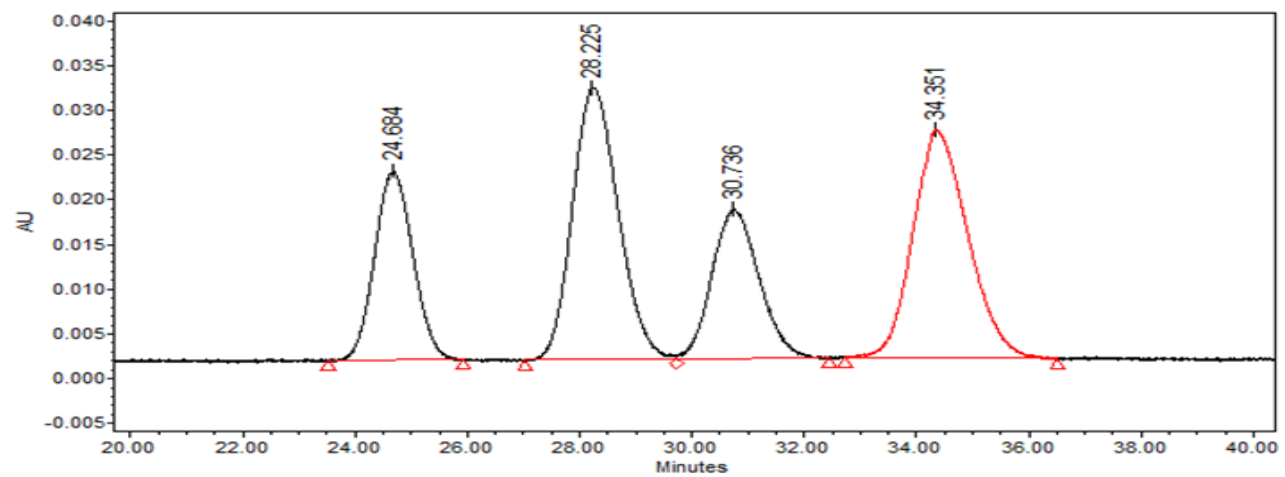

\begin{tabular}{c|r|r|r|}
\hline $\begin{array}{c}\text { Retention } \\
\text { Time } \\
(\mathrm{min})\end{array}$ & $\begin{array}{c}\text { Area } \\
(\mu \mathrm{V} \text { sec })\end{array}$ & \% Area & $\begin{array}{c}\text { Height } \\
(\mu \mathrm{V})\end{array}$ \\
\hline 24.684 & 997372 & 18.50 & 21219 \\
\hline 28.225 & 1711684 & 31.75 & 30331 \\
\hline 30.736 & 959872 & 17.80 & 16691 \\
\hline 34.351 & 1722923 & 31.95 & 25517 \\
\hline
\end{tabular}

Enantioenriched material: 98.5:1.5 er

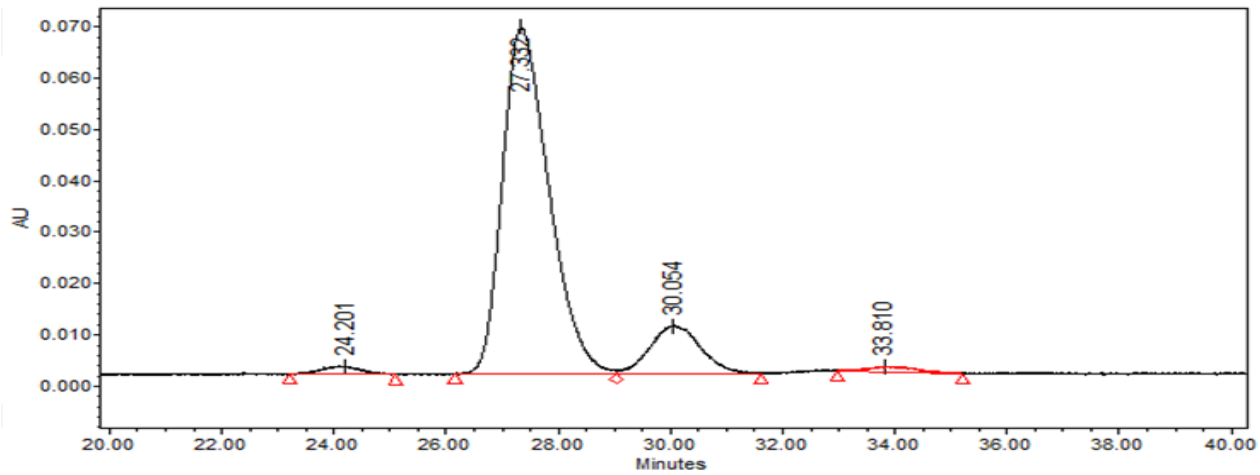

\begin{tabular}{c|r|r|r|}
\hline $\begin{array}{c}\text { Retention } \\
\text { Time } \\
(\mathrm{min})\end{array}$ & $\begin{array}{c}\text { Area } \\
\left(\mu \mathrm{V}^{*} \mathrm{sec}\right)\end{array}$ & \% Area & $\begin{array}{c}\text { Height } \\
(\mu \mathrm{V})\end{array}$ \\
\hline 24.201 & 74858 & 1.62 & 1671 \\
\hline 27.332 & 3881042 & 84.22 & 67426 \\
\hline 30.054 & 590309 & 12.81 & 9409 \\
\hline 33.810 & 62150 & 1.35 & 1119
\end{tabular}

(1S,2S)-2-methyl-1-(3-nitrophenyl)-2-((E)-2-(4,4,5,5-tetramethyl-1,3,2-dioxaborolan-2-yl)vinyl)hexan-1-ol (3h)

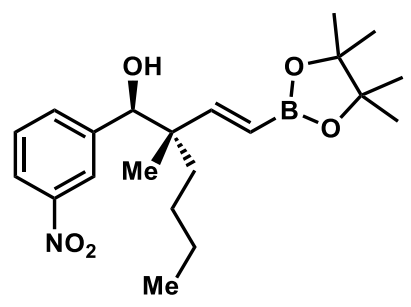

(3h)

Prepared according to General Method III. Purified by silica gel column chromatography (Hexanes/Ethyl Acetate 25:1, 20:1, 15:1, 10:1, 8:1, 5:1) to give the product (19.8 mg, 99\% yield, 99:1 er, 10:1 dr) as a colorless oil. ${ }^{1} \mathbf{H}$ NMR $\left(600 \mathrm{MHz}, \mathrm{CDCl}_{3}\right) \delta 8.15(\mathrm{~m}, 1 \mathrm{H}), 8.12(\mathrm{~m}, 1 \mathrm{H}), 7.61(\mathrm{~m}, 1 \mathrm{H})$, $7.49-7.44(\mathrm{t}, 7.9 \mathrm{~Hz}, 1 \mathrm{H}), 6.67-6.56(\mathrm{~d}, 18.5 \mathrm{~Hz}, 1 \mathrm{H}), 5.52-5.42(\mathrm{~d}, 18.5 \mathrm{~Hz}, 1 \mathrm{H}), 4.57$ (s, $1 \mathrm{H}), 2.18$ $(\mathrm{s}, 1 \mathrm{H}), 1.29(\mathrm{~s}, 12 \mathrm{H}), 1.25-1.09(\mathrm{~m}, 6 \mathrm{H}), 0.89(\mathrm{~s}, 3 \mathrm{H}), 0.87-0.83(\mathrm{t}, 7.4 \mathrm{~Hz}, 3 \mathrm{H}) .{ }^{13} \mathrm{C}$ NMR (151 $\left.\mathrm{MHz}_{1} \mathrm{CDCl}_{3}\right) \delta 157.8,147.8,142.7,134.3,128.4,123.1,122.7,83.5,79.2,47.4,37.0,26.4,25.0,24.9$, 
23.5, 16.7, 14.2. HRMS (m/z) calc. for $\left[\mathrm{M}+\mathrm{Na}^{+}\right] \mathrm{C}_{21} \mathrm{H}_{32} \mathrm{BNO}_{5} \mathrm{Na}^{+}: 412.2271$ found: 412.2260 . IR (cm1) $3458(\mathrm{w}), 2976(\mathrm{~m}), 2932(\mathrm{~m}), 1633(\mathrm{~m}), 1531(\mathrm{~s}), 1373(\mathrm{~s}), 1145(\mathrm{~m}), 969(\mathrm{w}), 851(\mathrm{w}) .[\alpha]_{\mathrm{D}^{23}}=-$ $15.4\left(\mathrm{c}=0.99, \mathrm{CH}_{2} \mathrm{Cl}_{2}, 1=1 \mathrm{dm}\right)$.

Diacel CHIRALPAK IC Column: 95:5 $\mathrm{CO}_{2}: \mathrm{iPrOH} ; 1.0 \mathrm{~mL} / \mathrm{min} ; 210 \mathrm{~nm}$.

\section{Racemic material:}

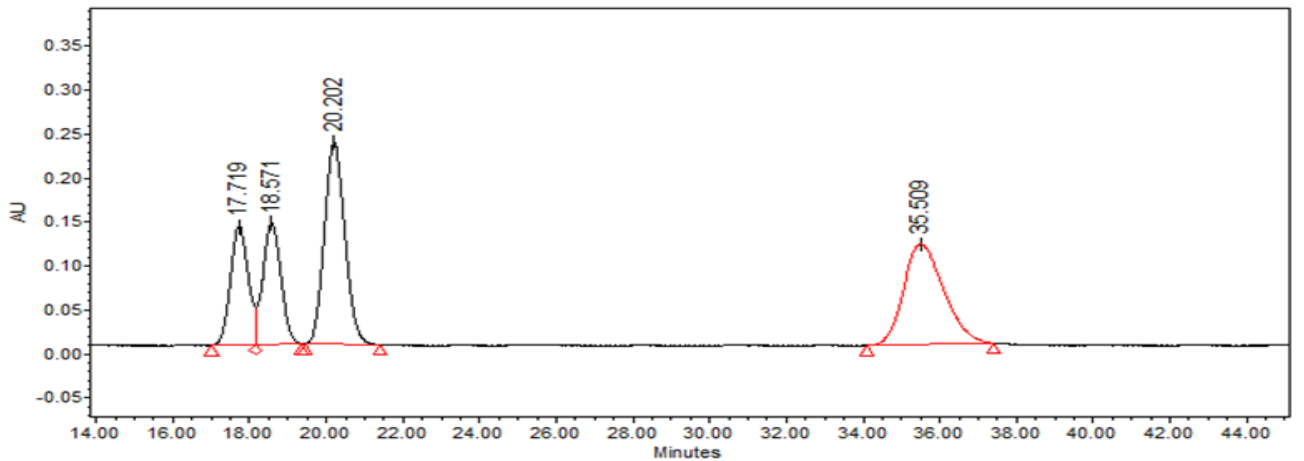

\begin{tabular}{c|c|c|c|}
\hline $\begin{array}{c}\text { Retention } \\
\text { Time } \\
(\mathrm{min})\end{array}$ & $\begin{array}{c}\text { Area } \\
\left(\mu \mathrm{V}^{*} \mathrm{sec}\right)\end{array}$ & $\%$ Area & $\begin{array}{c}\text { Height } \\
(\mu \mathrm{V})\end{array}$ \\
\hline 17.719 & 4347171 & 16.83 & 135383 \\
\hline 18.571 & 4583723 & 17.74 & 138507 \\
\hline 20.202 & 8571905 & 33.18 & 230889 \\
\hline 35.509 & 8332757 & 32.25 & 114824 \\
\hline
\end{tabular}

Enantioenriched material: 99:1 er

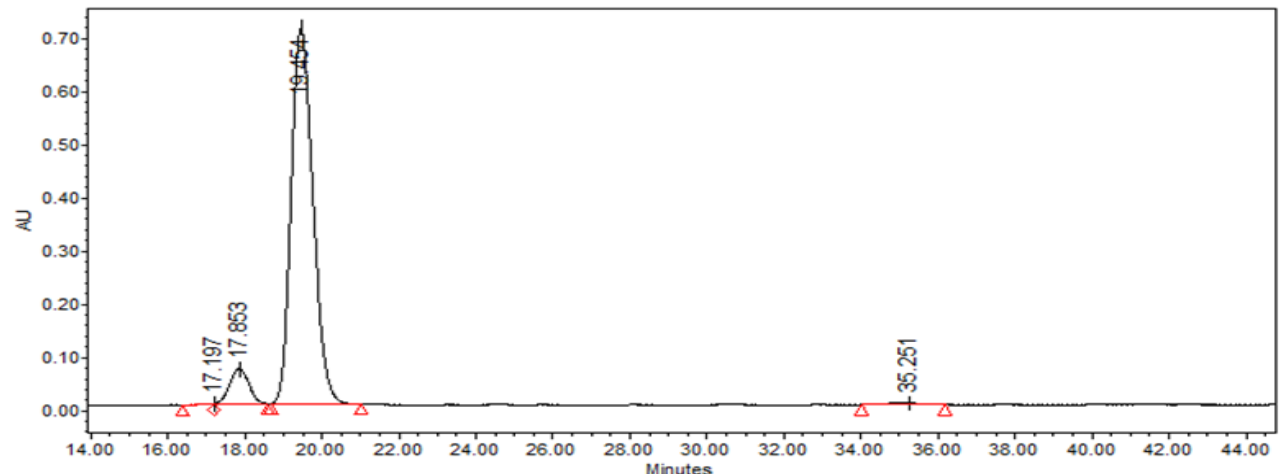

\begin{tabular}{c|r|r|r|}
\hline $\begin{array}{c}\text { Retention } \\
\text { Time } \\
(\mathrm{min})\end{array}$ & $\begin{array}{c}\text { Area } \\
\left(\mu \mathrm{V}^{*} \mathrm{sec}\right)\end{array}$ & \% Area & $\begin{array}{c}\text { Height } \\
(\mu \mathrm{V})\end{array}$ \\
\hline 17.197 & 29757 & 0.10 & 1791 \\
\hline 17.853 & 2296561 & 7.63 & 66737 \\
\hline 19.454 & 27548782 & 91.53 & 705918 \\
\hline 35.251 & 222357 & 0.74 & 3961 \\
\hline
\end{tabular}

(1S,2S)-2-methyl-2-((E)-2-(4,4,5,5-tetramethyl-1,3,2-dioxaborolan-2-yl)vinyl)-1-(3(trifluoromethyl)phenyl)hexan-1-ol (3i)

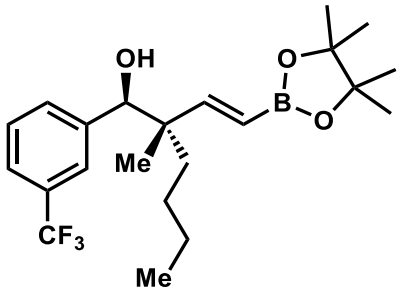

(3i)

Prepared according to General Method III. Purified by silica gel column chromatography (Hexanes/Ethyl Acetate 25:1, 20:1, 15:1, 10:1, 5:1) to give the product (16.1 mg, 73\% yield, 98.5:1.5 er, 12:1 dr) as a colorless oil. ${ }^{1} \mathbf{H}$ NMR $\left(600 \mathrm{MHz}^{\mathrm{CDCl}} 3\right) \delta 7.55(\mathrm{~s}, 1 \mathrm{H}), 7.53-7.50(\mathrm{~d}, 7.5 \mathrm{~Hz}, 1 \mathrm{H})$, $7.47-7.44(\mathrm{~d}, 7.9 \mathrm{~Hz}, 1 \mathrm{H}), 7.43-7.38(\mathrm{t}, 7.7 \mathrm{~Hz}, 1 \mathrm{H}), 6.65-6.58(\mathrm{~d}, 18.5 \mathrm{~Hz}, 1 \mathrm{H}), 5.50-5.42(\mathrm{~d}$, $18.5 \mathrm{~Hz}, 1 \mathrm{H}), 4.53(\mathrm{~s}, 1 \mathrm{H}), 2.06(\mathrm{~s}, 1 \mathrm{H}), 1.48-1.40(\mathrm{~m}, 1 \mathrm{H}), 1.29(\mathrm{~s}, 12 \mathrm{H}), 1.26-1.22(\mathrm{~m}, 3 \mathrm{H}), 1.16-$ 
$1.10(\mathrm{~m}, 2 \mathrm{H}), 0.89(\mathrm{~s}, 3 \mathrm{H}), 0.87-0.83(\mathrm{t}, 7.3 \mathrm{~Hz}, 3 \mathrm{H}) .{ }^{13} \mathrm{C}$ NMR $\left(151 \mathrm{MHz}, \mathrm{CDCl}_{3}\right) \delta$ 158.2, 141.6, 131.6, 130.1 (d, $32.4 \mathrm{~Hz}), 127.9,124.9$ (q, 3.9 Hz), 124.4 (q, 3.9 Hz), 124.4 (d, 272.4 Hz), 83.5, 79.6,

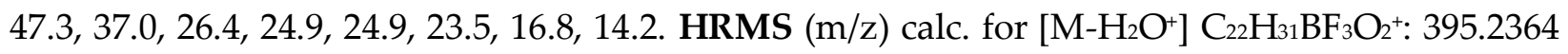
found: 395.2361 . IR ( $\left.\mathrm{cm}^{-1}\right) 2929$ (w), 1585 (m), 1483 (s), 1451 (s), 1427 (s), 1260 (s), 1186 (s), 1063 (s), $950(\mathrm{~m}), 746(\mathrm{w}) .[\alpha]_{\mathrm{D}^{23}}=-19.8\left(\mathrm{c}=0.81, \mathrm{CH}_{2} \mathrm{Cl}_{2}, \mathrm{l}=1 \mathrm{dm}\right)$.

Phenomenex lux Cellulose OX-H Column: $98.5: 1.5 \mathrm{CO}_{2}: \mathrm{MeOH} ; 1.0 \mathrm{~mL} / \mathrm{min} ; 210 \mathrm{~nm}$.

\section{Racemic material:}

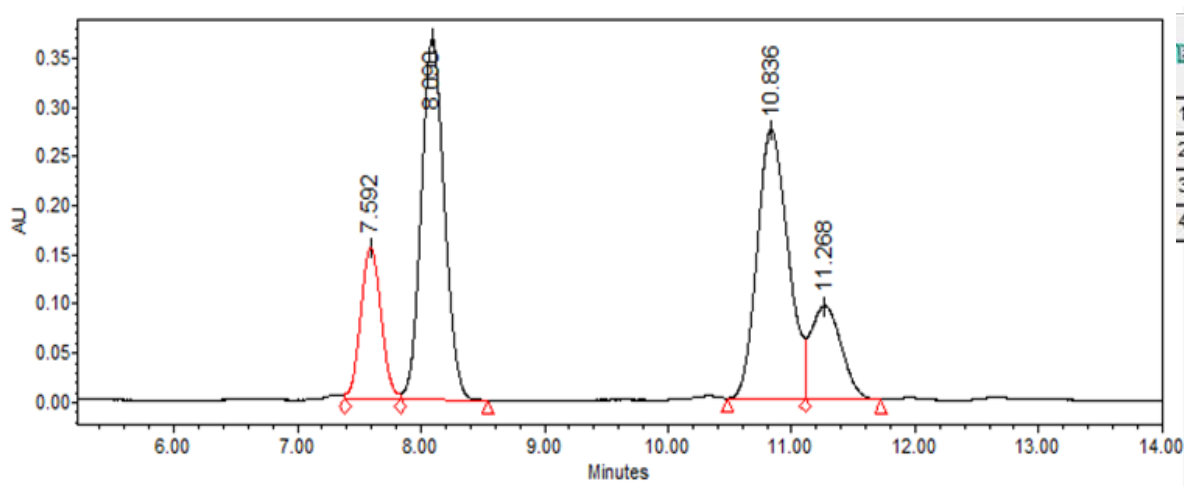

\begin{tabular}{|c|c|c|c|c|}
\hline 国 & $\begin{array}{c}\text { Retention } \\
\text { Time } \\
(\mathrm{min})\end{array}$ & $\begin{array}{c}\text { Area } \\
\left(\mu \mathrm{V}^{*} \mathrm{sec}\right)\end{array}$ & $\%$ Area & $\begin{array}{c}\text { Height } \\
(\mu \mathrm{V})\end{array}$ \\
\hline 1 & 7.592 & 1779518 & 13.75 & 155062 \\
\hline 2 & 8.090 & 4772649 & 36.87 & 367579 \\
\hline 3 & 10.836 & 4745795 & 36.67 & 273391 \\
\hline 4 & 11.268 & 1645466 & 12.71 & 95064 \\
\hline
\end{tabular}

Enantioenriched material: 98.5:1.5 er

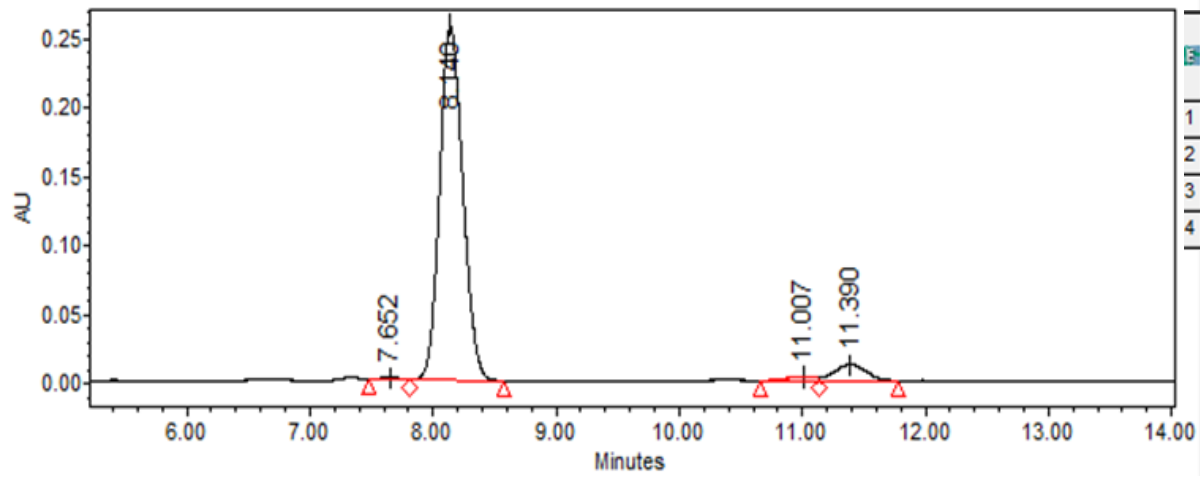

\begin{tabular}{|l|r|r|r|r|}
\hline 国 & $\begin{array}{c}\text { Retention } \\
\text { Time } \\
(\mathrm{min})\end{array}$ & $\begin{array}{c}\text { Area } \\
\left(\mu \mathrm{V}^{*} \mathrm{sec}\right)\end{array}$ & $\%$ Area & $\begin{array}{c}\text { Height } \\
(\mu \mathrm{V})\end{array}$ \\
\hline 1 & 7.652 & 19866 & 0.55 & 2076 \\
\hline 2 & 8.140 & 3342350 & 92.38 & 256282 \\
\hline 3 & 11.007 & 46619 & 1.29 & 2951 \\
\hline 4 & 11.390 & 209235 & 5.78 & 11785 \\
\hline
\end{tabular}

(1S,2S)-1-(2-bromo-4-chlorophenyl)-2-methyl-2-((E)-2-(4,4,5,5-tetramethyl-1,3,2-dioxaborolan-2yl)vinyl)hexan-1-ol (3j)

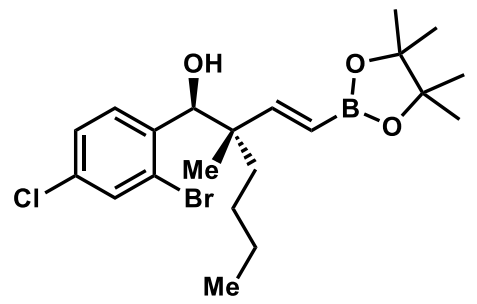

(3j)

Prepared according to General Method III. Purified by silica gel column chromatography (Hexanes/Ethyl Acetate 25:1, 20:1, 15:1, 10:1, 8:1, 5:1) to give the product (17.8 mg, 78\% yield, 
98.5:1.5 er, 7:1 dr) as a colorless oil. ${ }^{1} \mathbf{H}$ NMR $\left(600 \mathrm{MHz}, \mathrm{CDCl}_{3}\right) \delta 7.54-7.50(\mathrm{~m}, 1 \mathrm{H}), 7.41-7.37$ $(\mathrm{m}, 1 \mathrm{H}), 7.26-7.22(\mathrm{~m}, 1 \mathrm{H}), 6.74-6.68(\mathrm{~d}, 18.5 \mathrm{~Hz}, 1 \mathrm{H}), 5.48-5.41(\mathrm{~d}, 18.5 \mathrm{~Hz}, 1 \mathrm{H}), 5.03(\mathrm{~s}, 1 \mathrm{H})$, $1.74-1.67(\mathrm{~m}, 1 \mathrm{H}), 1.29(\mathrm{~s}, 12 \mathrm{H}), 1.26-1.21(\mathrm{~m}, 3 \mathrm{H}), 1.15-1.05(\mathrm{~m}, 2 \mathrm{H}), 0.95(\mathrm{~s}, 3 \mathrm{H}), 0.87-0.83(\mathrm{t}$, $7.3 \mathrm{~Hz}, 3 \mathrm{H}) .{ }^{13} \mathrm{C}$ NMR $\left(151 \mathrm{MHz}, \mathrm{CDCl}_{3}\right) \delta$ 158.3, 138.8, 133.9, 132.0, 131.1, 127.2, 124.6, 83.4, 76.7,

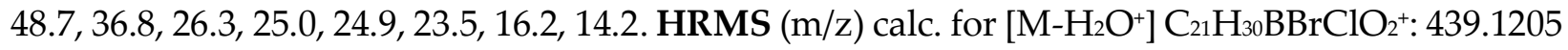
found: 439.1201. IR ( $\left.\mathrm{cm}^{-1}\right) 3459$ (s), 2957 (s), 2930 (s), 2859 (m), 2360 (w), 1633 (m), 1468 (m), 1351 (s), $1145(\mathrm{~s}), 1029(\mathrm{w}), 851(\mathrm{w}) .[\alpha]_{\mathrm{D}^{23}}-33.9\left(\mathrm{c}=0.89, \mathrm{CH}_{2} \mathrm{Cl}_{2}, \mathrm{l}=1 \mathrm{dm}\right)$.

Phenomenex lux Cellulose 1-OD Column: $98: 2 \mathrm{CO}_{2}: \mathrm{PrOH} ; 1.0 \mathrm{~mL} / \mathrm{min} ; 210 \mathrm{~nm}$.

\section{Racemic material:}
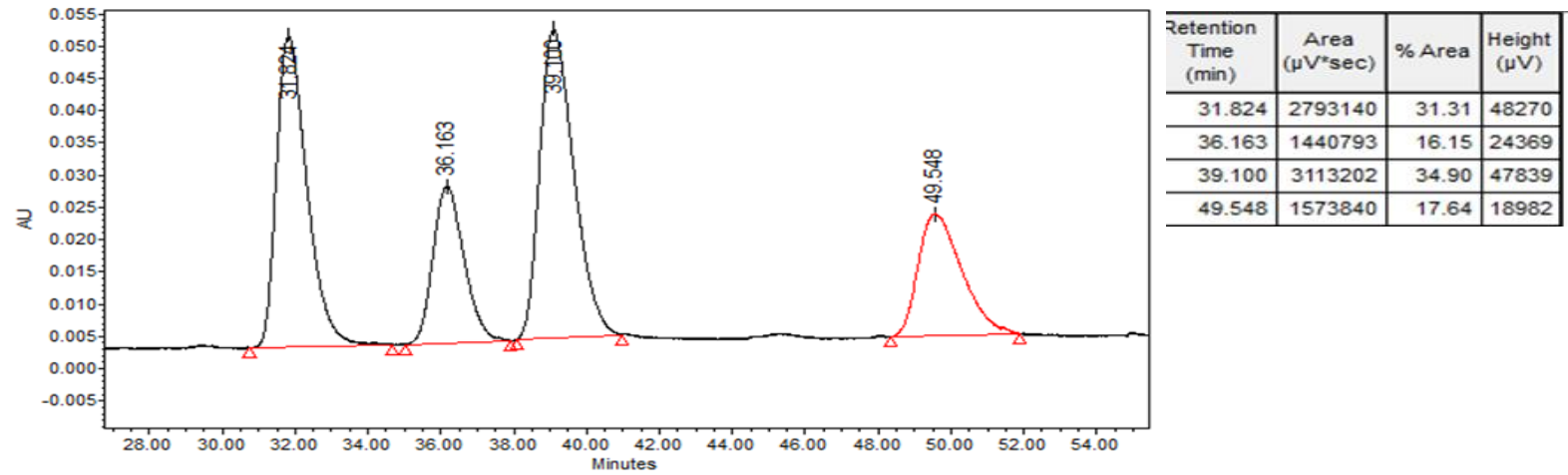

Enantioenriched material: 98.5:1.5 er

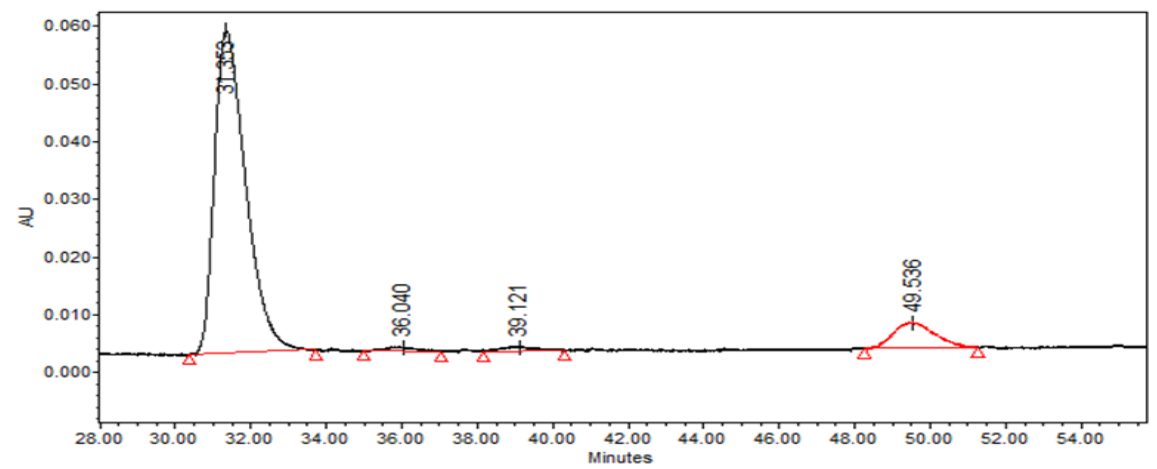

\begin{tabular}{c|r|r|r|}
\hline $\begin{array}{c}\text { Retention } \\
\text { Time } \\
(\mathrm{min})\end{array}$ & $\begin{array}{c}\text { Area } \\
\left(\mu \mathrm{V}^{*} \mathrm{sec}\right)\end{array}$ & \% Area & $\begin{array}{c}\text { Height } \\
(\mu \mathrm{V})\end{array}$ \\
\hline 31.353 & 3204377 & 88.78 & 55799 \\
\hline 36.040 & 31350 & 0.87 & 704 \\
\hline 39.121 & 42186 & 1.17 & 764 \\
\hline 49.536 & 331322 & 9.18 & 4438 \\
\hline
\end{tabular}

(1S,2S)-1-(2,6-dichloropyridin-3-yl)-2-methyl-2-((E)-2-(4,4,5,5-tetramethyl-1,3,2-dioxaborolan-2yl)vinyl)hexan-1-ol (3k)<smiles>CCCC[C@](C)(/C=C/B1OC(C)(C)C(C)(C)O1)[C@H](O)c1ccc(Cl)nc1Cl</smiles>

(3k) 
Prepared according to General Method III. Purified by silica gel column chromatography (Hexanes/Ethyl Acetate 15:1, 10:1, 8:1, 5:1) to give the product (23 mg, 76\% yield, 97.5:2.5 er, 4:1 dr) as a colorless oil.

\section{Major Diastereomer}

${ }^{1} \mathrm{H}$ NMR $\left(600 \mathrm{MHz}, \mathrm{CDCl}_{3}\right) \delta 7.80-7.75(\mathrm{~d}, 8.1 \mathrm{~Hz}, 1 \mathrm{H}), 7.24-7.22(\mathrm{~d}, 8.1 \mathrm{~Hz}, 1 \mathrm{H}), 6.72$ - $6.65(\mathrm{~d}$, $18.5 \mathrm{~Hz}, 1 \mathrm{H}), 5.47-5.40(\mathrm{~d}, 18.5 \mathrm{~Hz}, 1 \mathrm{H}), 5.01(\mathrm{~s}, 1 \mathrm{H}), 2.23(\mathrm{~s}, 1 \mathrm{H}), 1.70-1.64(\mathrm{~m}, 1 \mathrm{H}), 1.28(\mathrm{~s}, 12 \mathrm{H})$, $1.25-1.08(\mathrm{~m}, 5 \mathrm{H}), 0.93(\mathrm{~s}, 3 \mathrm{H}), 0.88-0.82(\mathrm{t}, 7.1 \mathrm{~Hz}, 3 \mathrm{H}) .{ }^{13} \mathrm{C}$ NMR $\left(151 \mathrm{MHz}, \mathrm{CDCl}_{3}\right) \delta 157.5$, $149.3,149.1,141.5,134.4,122.7,83.5,74.3,48.6,36.9,26.3,25.0,24.9,23.5,16.2,14.2$.

\section{Minor Diastereomer}

${ }^{1} \mathrm{H}$ NMR $\left(600 \mathrm{MHz}, \mathrm{CDCl}_{3}\right) \delta 7.80$ - $7.75(\mathrm{~d}, 8.1 \mathrm{~Hz}, 1 \mathrm{H}), 7.24-7.22(\mathrm{~d}, 8.1 \mathrm{~Hz}, 1 \mathrm{H}), 6.63$ - $6.58(\mathrm{~d}$, $18.4 \mathrm{~Hz}, 1 \mathrm{H}), 5.34-5.29(\mathrm{~d}, 18.5 \mathrm{~Hz}, 1 \mathrm{H}), 4.98(\mathrm{~s}, 1 \mathrm{H}), 2.29(\mathrm{~s}, 1 \mathrm{H}), 1.70-1.64(\mathrm{~m}, 1 \mathrm{H}), 1.26(\mathrm{~s}, 12 \mathrm{H})$, $1.25-1.08(\mathrm{~m}, 5 \mathrm{H}), 1.05(\mathrm{~s}, 3 \mathrm{H}), 0.88-0.82(\mathrm{t}, 7.1 \mathrm{~Hz}, 3 \mathrm{H}) .{ }^{13} \mathrm{C}$ NMR $\left(151 \mathrm{MHz}, \mathrm{CDCl}_{3}\right) \delta 156.68$, 149.10, 148.97, 141.22, 134.80, 122.66, 83.45, 74.94, 47.99, 35.70, 26.35, 25.09, 24.72, 23.60, 17.77, 14.21 .

HRMS (m/z) calc. for $\left[\mathrm{M}+\mathrm{H}^{+}\right] \mathrm{C}_{20} \mathrm{H}_{31} \mathrm{BCl}_{2} \mathrm{NO}_{3}{ }^{+}$: 414.1769 found: 414.1772 . IR $\left(\mathrm{cm}^{-1}\right) 3442(\mathrm{w}), 2977$ (m), $2932(\mathrm{~m}), 2861(\mathrm{w}), 1633(\mathrm{~m}), 1550(\mathrm{~m}), 1351(\mathrm{~s}), 1144(\mathrm{~s}), 970(\mathrm{w}), 851(\mathrm{w}) .[\alpha]_{\mathrm{D}^{23}}=-27.2(\mathrm{c}=$ $\left.1.15, \mathrm{CH}_{2} \mathrm{Cl}_{2}, \mathrm{l}=1 \mathrm{dm}\right)$.

Diacel CHIRALPAK IA Column: 97:3 hexanes:iPrOH; $0.5 \mathrm{~mL} / \mathrm{min} ; 210 \mathrm{~nm}$.

\section{Racemic material:}

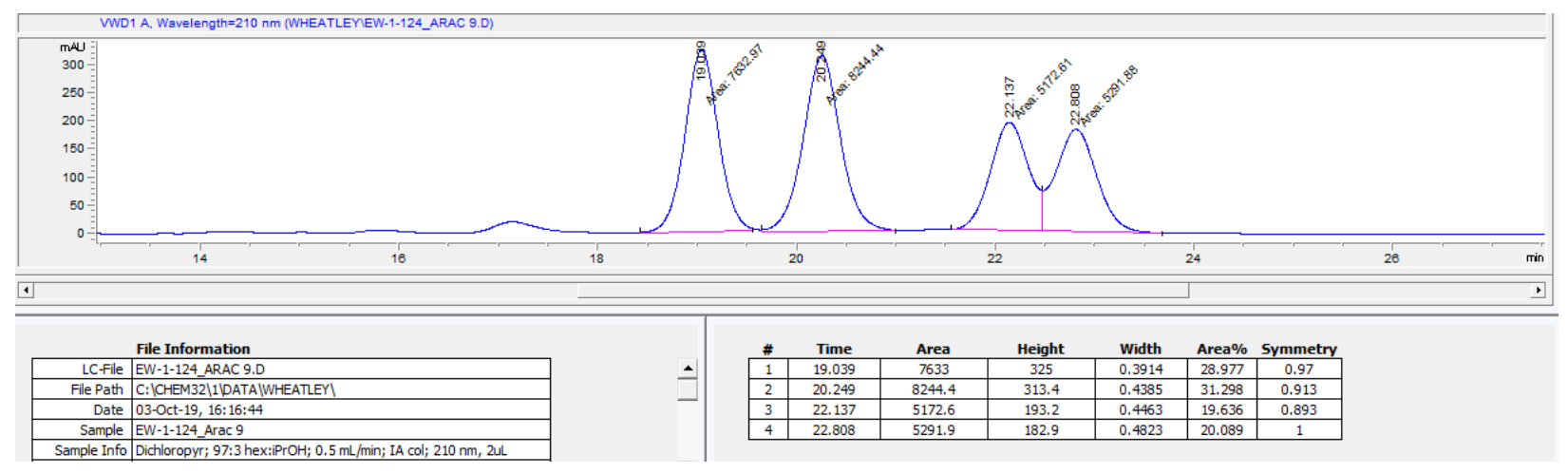

\section{Enantioenriched material:}

Major: 97.5:2.5 er

Minor: >99:1 er 


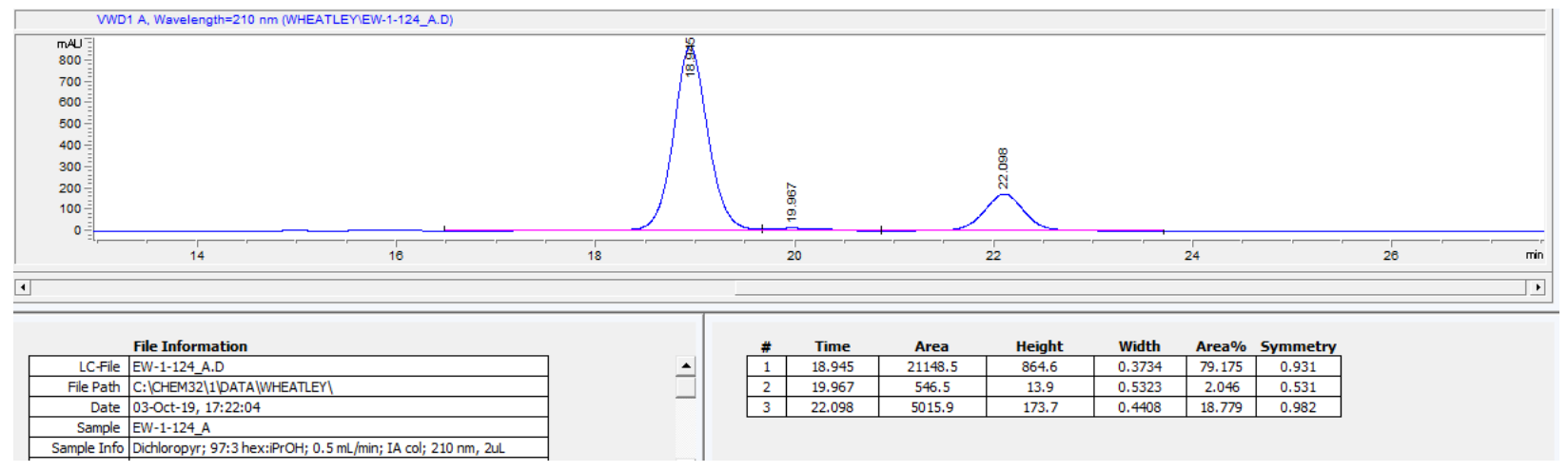

(1S,2S)-1-(furan-2-yl)-2-methyl-2-((E)-2-(4,4,5,5-tetramethyl-1,3,2-dioxaborolan-2-yl)vinyl)hexan-1-ol (3I)

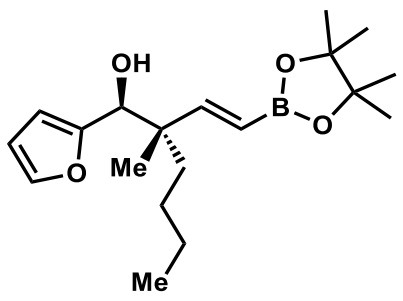

(3I)

Prepared according to General Method III. Purified by silica gel column chromatography (Hexanes/Ethyl Acetate 25:1, 20:1, 15:1, 10:1, 8:1) to give the product (14.7 mg, 87\% yield, 99:1 er, 4:1 dr) as a colorless oil.

\section{Major Diastereomer}

${ }^{1} \mathbf{H}$ NMR (400 MHz, $\left.\mathrm{CDCl}_{3}\right) \delta 7.38-7.34(\mathrm{~d}, 1.7 \mathrm{~Hz}, 1 \mathrm{H}), 6.68$ - 6.58 (d, $\left.18.5 \mathrm{~Hz}, 1 \mathrm{H}\right), 6.41$ - 6.32 $(\mathrm{dd}, 3.2,1.8 \mathrm{~Hz}, 1 \mathrm{H}), 6.27-6.21$ (d, $3.3 \mathrm{~Hz}, 1 \mathrm{H}), 5.58-5.49$ (d, $18.5 \mathrm{~Hz}, 1 \mathrm{H}), 4.57-4.51$ (d, $2.9 \mathrm{~Hz}$, $1 \mathrm{H}), 1.99(\mathrm{~s} 1 \mathrm{H}), 1.42-1.34(\mathrm{~m}, 2 \mathrm{H}), 1.28(\mathrm{~s}, 12 \mathrm{H}), 1.26-1.10(\mathrm{~m}, 4 \mathrm{H}), 1.00(\mathrm{~s}, 3 \mathrm{H}), 0.88-0.80(\mathrm{t}$, $7.2 \mathrm{~Hz}, 3 \mathrm{H}) .{ }^{13} \mathrm{C}$ NMR $\left(101 \mathrm{MHz}, \mathrm{CDCl}_{3}\right) \delta$ 158.6, 154.4, 141.7, 110.2, 107.9, 83.4, 74.1, 47.2, 37.1, 26.2, 24.9, 24.9, 23.5, 17.0.

\section{Minor Diastereomer}

${ }^{1} \mathbf{H}$ NMR $\left(600 \mathrm{MHz}, \mathrm{CDCl}_{3}\right) \delta 7.34-7.33(\mathrm{~d}, 1.4 \mathrm{~Hz}, 1 \mathrm{H}), 6.68-6.58(\mathrm{~d}, 18.5 \mathrm{~Hz}, 1 \mathrm{H}), 6.32-6.30$ $(\mathrm{dd}, 3.21-1.8 \mathrm{~Hz}, 1 \mathrm{H}), 6.20-6.16(\mathrm{~d}, 3.2 \mathrm{~Hz}, 1 \mathrm{H}), 5.50-5.43(\mathrm{~d}, 18.5 \mathrm{~Hz}, 1 \mathrm{H}), 4.46(\mathrm{~s}, 1 \mathrm{H}), 2.06(\mathrm{~s}$, $1 \mathrm{H}), 1.42-1.34(\mathrm{~m}, 2 \mathrm{H}), 1.27(\mathrm{~s}, 12 \mathrm{H}), 1.26-1.10(\mathrm{~m}, 4 \mathrm{H}), 1.07(\mathrm{~s}, 1 \mathrm{H}), 0.88-0.80(\mathrm{t}, 7.2 \mathrm{~Hz}, 3 \mathrm{H})$. ${ }^{13} \mathrm{C}$ NMR $\left(151 \mathrm{MHz}, \mathrm{CDCl}_{3}\right) \delta$ 157.8, 154.7, 141.6, 110.1, 107.7, 83.3, 74.8, 46.8, 36.0, 26.3, 25.0, 24.8, 23.6, 19.1 .

HRMS (m/z) calc. for [M+Na+] $\mathrm{C}_{19} \mathrm{H}_{31} \mathrm{BO}_{4} \mathrm{Na}^{+}$: 357.2213 found: 357.2202. IR (cm-1) $3466(\mathrm{w}), 2960$ $(\mathrm{m}), 2932(\mathrm{~m}), 2872(\mathrm{w}), 1633(\mathrm{~m}), 1379(\mathrm{~s}), 1146(\mathrm{~s}), 1008(\mathrm{w}), 730(\mathrm{w}) .[\alpha]_{\mathrm{D}^{23}}=-27.6(\mathrm{c}=0.75$, $\left.\mathrm{CH}_{2} \mathrm{Cl}_{2}, \mathrm{l}=1 \mathrm{dm}\right)$.

Diacel CHIRALPAK IA Column: 97:3 hexanes:iPrOH; $0.5 \mathrm{~mL} / \mathrm{min} ; 210 \mathrm{~nm}$. 


\section{Racemic material:}

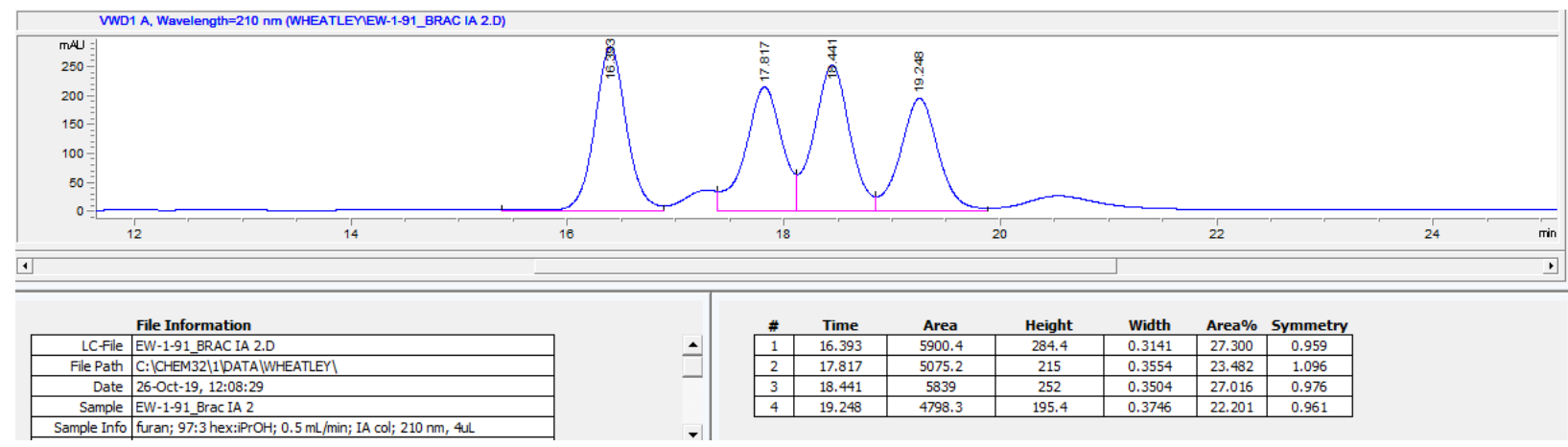

\section{Enantioenriched material:}

Major: 99:1 er

Minor: $94: 6$ er

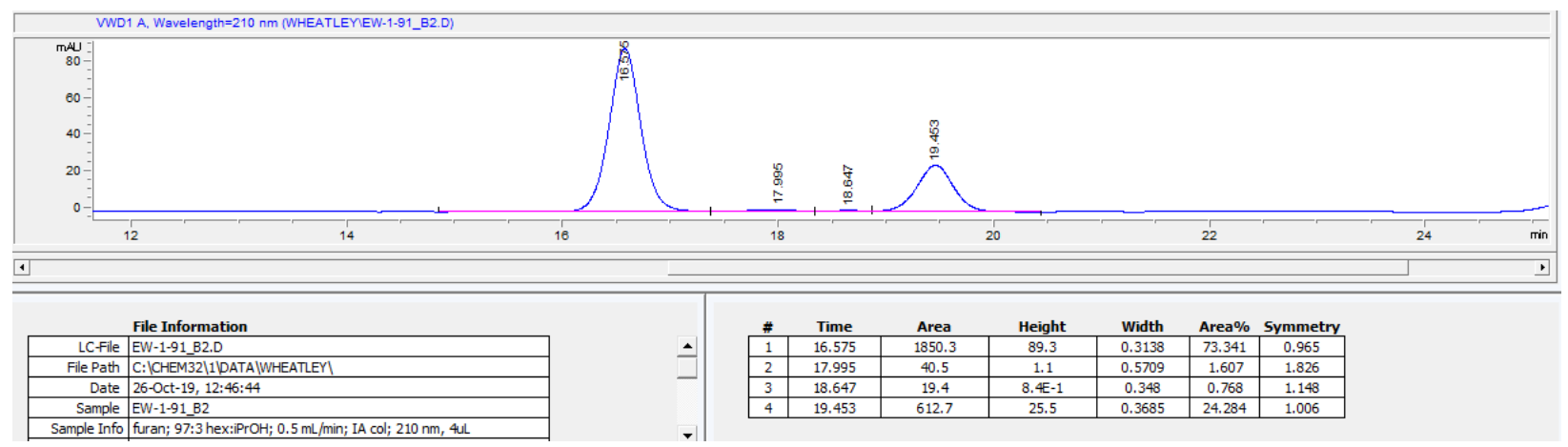

(1S,2S)-2-methyl-2-((E)-2-(4,4,5,5-tetramethyl-1,3,2-dioxaborolan-2-yl)vinyl)-1-(thiophen-2-yl)hexan-1-ol $(3 \mathrm{~m})$

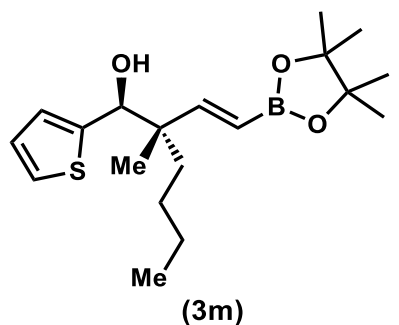

Prepared according to General Method III. Purified by silica gel column chromatography (Hexanes/Ethyl Acetate 25:1, 20:1, 15:1, 10:1, 8:1) to give the product (18.0 mg, 73\% yield, >99:1 er, 3:1 dr) as a colorless oil.

\section{Major Diastereomer}

${ }^{1} \mathrm{H}$ NMR $\left(600 \mathrm{MHz}, \mathrm{CDCl}_{3}\right) \delta 7.25-7.22(\mathrm{dd}, 4.9 \mathrm{~Hz}, 1.3 \mathrm{~Hz}, 1 \mathrm{H}), 6.97-6.93(\mathrm{~m}, 2 \mathrm{H}), 6.70-6.64$ $(\mathrm{d}, 18.5 \mathrm{~Hz}, 1 \mathrm{H}), 5.58-5.53(\mathrm{~d}, 18.5 \mathrm{~Hz}, 1 \mathrm{H}), 4.77(\mathrm{~s}, 1 \mathrm{H}), 2.08(\mathrm{~s}, 1 \mathrm{H}), 1.47-1.37(\mathrm{~m}, 2 \mathrm{H}), 1.29$ (s, $12 \mathrm{H}), 1.26-1.21(\mathrm{~m}, 2 \mathrm{H}), 1.18-1.11(\mathrm{~m}, 2 \mathrm{H}), 0.99(\mathrm{~s}, 3 \mathrm{H}), 0.87-0.82(\mathrm{t}, 7.3 \mathrm{~Hz}, 3 \mathrm{H}) .{ }^{13} \mathrm{C}$ NMR $(151$ $\left.\mathrm{MHz}_{2} \mathrm{CDCl}_{3}\right) \delta 158.7,144.1,126.1,125.9,124.8,83.4,76.6,47.3,37.4,26.4,25.0,24.9,23.5,16.8,14.2$. 


\section{Minor Diastereomer}

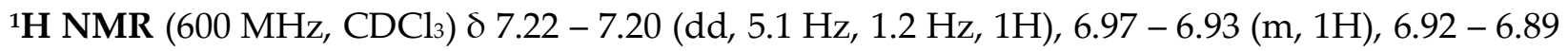
$(\mathrm{m}, 1 \mathrm{H}), 6.70-6.63(\mathrm{~d}, 18.6 \mathrm{~Hz}, 1 \mathrm{H}), 5.50-5.45(\mathrm{~d}, 18.5 \mathrm{~Hz}, 1 \mathrm{H}), 4.75(\mathrm{~s}, 1 \mathrm{H}), 2.12(\mathrm{~s}, 1 \mathrm{H}), 1.47-$ $1.37(\mathrm{~m}, 2 \mathrm{H}), 1.28(\mathrm{~s}, 12 \mathrm{H}), 1.26-1.21(\mathrm{~m}, 2 \mathrm{H}), 1.18-1.11(\mathrm{~m}, 2 \mathrm{H}), 1.06(\mathrm{~s}, 3 \mathrm{H}), 0.87-0.82(\mathrm{t}, 7.3$ $\mathrm{Hz}, 3 \mathrm{H}) .{ }^{13} \mathrm{C}$ NMR $\left(151 \mathrm{MHz}, \mathrm{CDCl}_{3}\right) \delta$ 158.0, 145.0, 126.2, 125.5, 124.5, 83.3, 76.6, 46.7, 35.8, 26.4, 25.0, 24.8, 23.6, 19.2, 14.2.

HRMS (m/z) calc. for [M- $\left.\mathrm{H}_{2} \mathrm{O}^{+}\right] \mathrm{C}_{19} \mathrm{H}_{30} \mathrm{BO}_{2} \mathrm{~S}^{+}: 333.2054$ found: 333.2056. IR $\left(\mathrm{cm}^{-1}\right) 3466(\mathrm{w}), 2976$ (m), $2931(\mathrm{~m}), 2861(\mathrm{w}), 1633(\mathrm{~m}), 1372(\mathrm{~s}), 1351(\mathrm{~s}), 1146(\mathrm{~s}), 851(\mathrm{w}), 696(\mathrm{w}) .[\alpha]_{\mathrm{D}}^{23}=-10.1(\mathrm{c}=$ $\left.0.9, \mathrm{CH}_{2} \mathrm{Cl}_{2}, \mathrm{l}=1 \mathrm{dm}\right)$.

Phenomenex lux Amylose 1-AD Column: 97:3 $\mathrm{CO}_{2}: \mathrm{iPrOH} ; 1.0 \mathrm{~mL} / \mathrm{min} ; 210 \mathrm{~nm}$.

Racemic material:

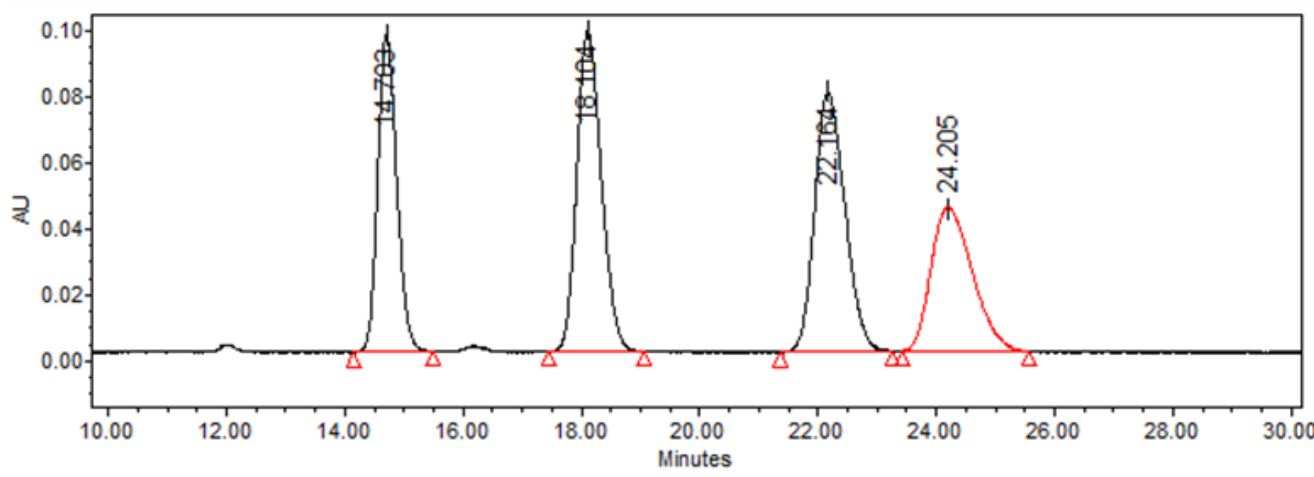

\begin{tabular}{|c|c|c|c|}
\hline $\begin{array}{c}\text { Retention } \\
\text { Time } \\
(\mathrm{min})\end{array}$ & $\begin{array}{c}\text { Area } \\
\left(\mu \mathrm{V}^{*} \mathrm{sec}\right)\end{array}$ & $\%$ Area & $\begin{array}{c}\text { Height } \\
(\mu \mathrm{V})\end{array}$ \\
\hline 14.703 & 2139444 & 21.74 & 95878 \\
\hline 18.104 & 2792684 & 28.38 & 97048 \\
\hline 22.164 & 2793840 & 28.39 & 78660 \\
\hline 24.205 & 2114566 & 21.49 & 43523 \\
\hline
\end{tabular}

Enantioenriched material:

Major: >99:1 er

Minor: >99:1 er

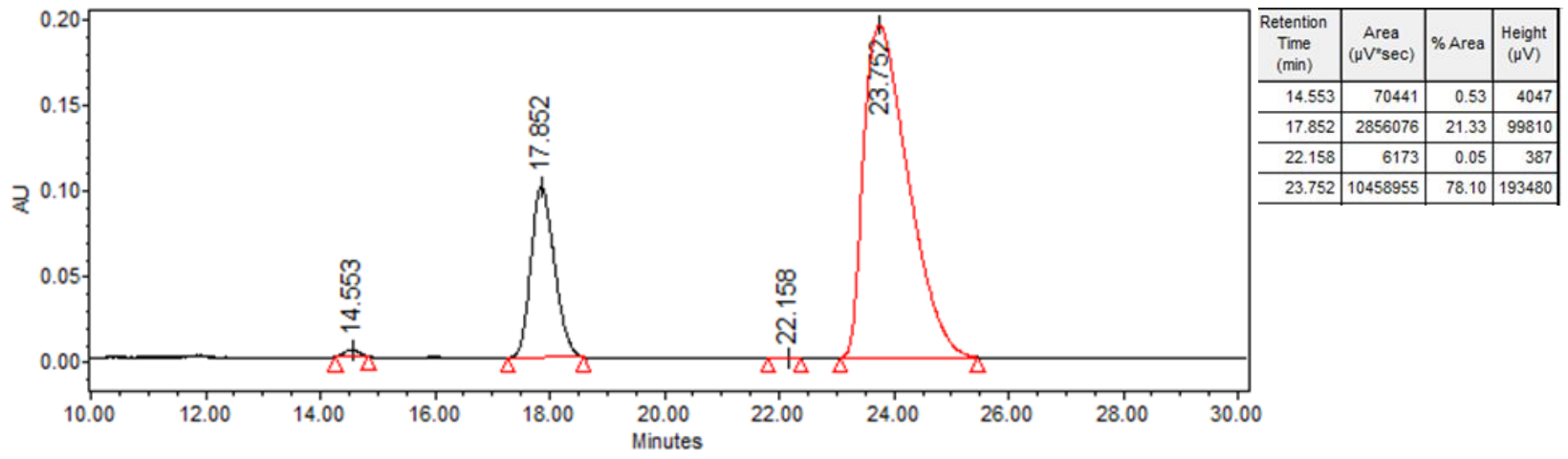

tert-butyl-3-((1S,2S)-1-hydroxy-2-methyl-2-((E)-2-(4,4,5,5-tetramethyl-1,3,2-dioxaborolan-2yl)vinyl)hexyl)-1H-indole-1-carboxylate (3n) 


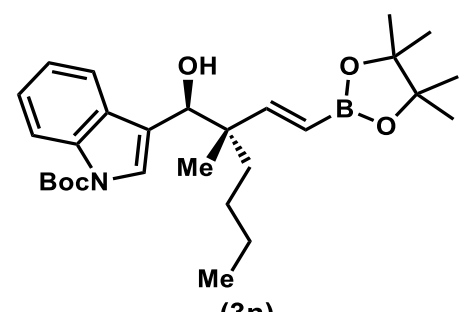

(3n)

Prepared according to General Method III. Purified by silica gel column chromatography (Hexanes/Ethyl Acetate 25:1, 20:1, 15:1, 10:1, 8:1) to give the product (19.1 mg, 78\% yield, >99:1 er, $15: 1 \mathrm{dr})$ as a white solid (melting point $\left.=56-60{ }^{\circ} \mathrm{C}\right) .{ }^{1} \mathbf{H} \mathbf{~ N M R}\left(600 \mathrm{MHz}, \mathrm{CDCl}_{3}\right) \delta 8.12(\mathrm{~s}, 1 \mathrm{H}), 7.64$ -7.59 (d, $7.9 \mathrm{~Hz}, 1 \mathrm{H}), 7.52$ (s, 1H), $7.31-7.26$ (t, $8.2 \mathrm{~Hz}, 1 \mathrm{H}), 7.22-7.18$ (t, $7.6 \mathrm{~Hz}, 1 \mathrm{H}), 6.74-6.69$ $(\mathrm{d}, 18.5 \mathrm{~Hz}, 1 \mathrm{H}), 5.59-5.52(\mathrm{~d}, 18.5 \mathrm{~Hz}, 1 \mathrm{H}), 4.82(\mathrm{~s}, 1 \mathrm{H}), 1.69-1.65(\mathrm{~s}, 9 \mathrm{H}), 1.29(\mathrm{~s}, 12 \mathrm{H}), 1.24-$ $1.18(\mathrm{~m}, 3 \mathrm{H}), 1.16-1.10(\mathrm{~m}, 3 \mathrm{H}), 1.05(\mathrm{~s}, 3 \mathrm{H}), 0.85-0.79(\mathrm{t}, 7.2 \mathrm{~Hz}, 3 \mathrm{H}) .{ }^{13} \mathrm{C} \mathrm{NMR}\left(151 \mathrm{MHz}, \mathrm{CDCl}_{3}\right)$ ठ 159.3, 130.2, 124.3, 124.2, 122.5, 120.8, 120.7, 115.2, 83.4, 74.0, 47.9, 37.1, 28.3, 26.4, 25.0, 24.9, 24.7, 23.5, 16.9, 14.2. HRMS (m/z) calc. for $\left[\mathrm{M}-\mathrm{H}_{2} \mathrm{O}^{+}\right] \mathrm{C}_{28} \mathrm{H}_{41} \mathrm{BNO}_{4}{ }^{+}: 466.3123$ found: 466.3120 . IR $\left(\mathrm{cm}^{-1}\right)$ 2977 (m), 2931 (m), 2861 (w), 1735 (s), 1634 (m), 1454 (m), 1372 (s), 1351 (s), 1273 (w), 1158 (m), $1082(\mathrm{w}), 901(\mathrm{w}) .[\alpha]_{\mathrm{D}^{23}}-8.7\left(\mathrm{c}=0.86, \mathrm{CH}_{2} \mathrm{Cl}_{2}, \mathrm{l}=1 \mathrm{dm}\right)$.

Diacel CHIRALPAK IC Column: 98:2 CO $: \mathrm{PPrOH} ; 1.0 \mathrm{~mL} / \mathrm{min} ; 210 \mathrm{~nm}$.

\section{Racemic material:}
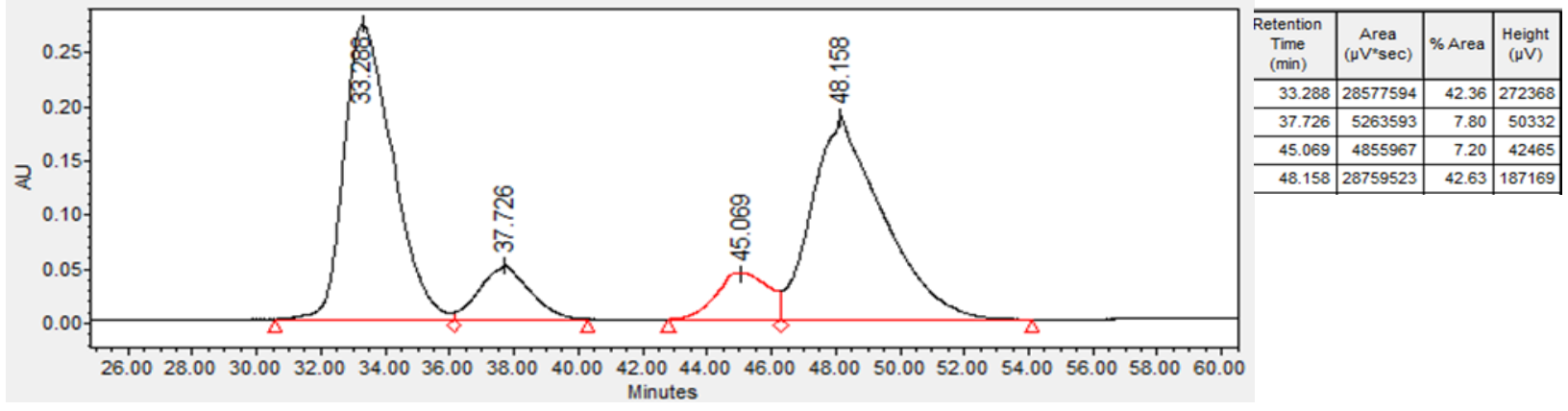

Enantioenriched material: >99:1 er
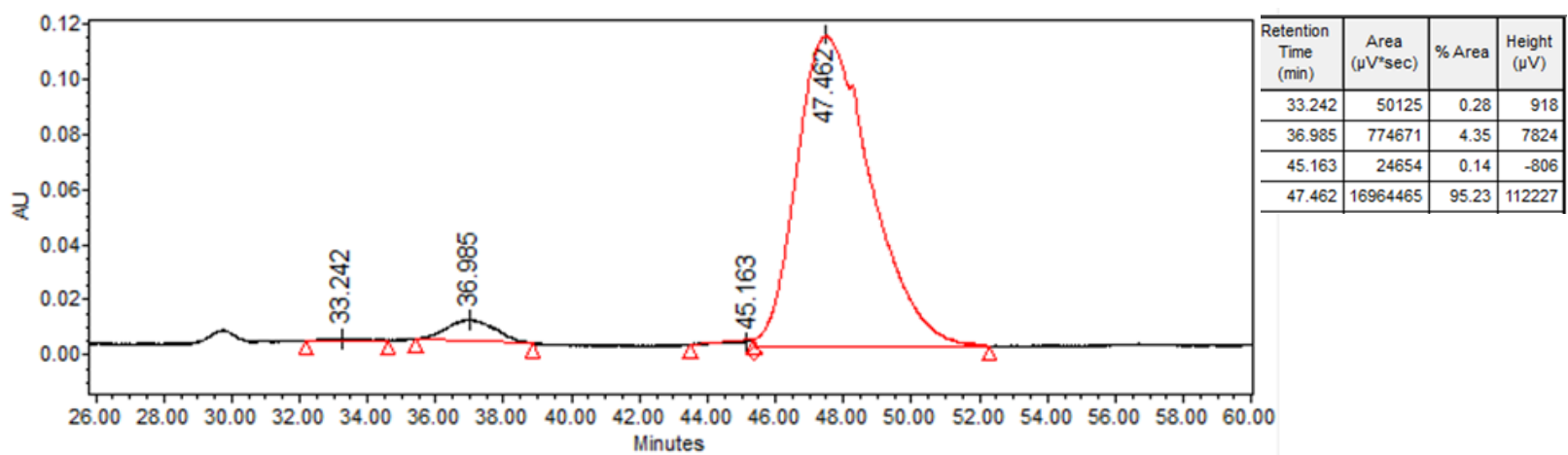
tert-butyl-3-((1S,2S)-1-hydroxy-2-methyl-2-((E)-2-(4,4,5,5-tetramethyl-1,3,2-dioxaborolan-2yl)vinyl)hexyl)-1H-indole-1-carboxylate (3n)

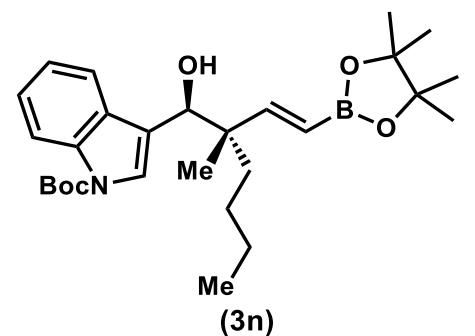

Prepared according to General Method III from tert-butyl 3-formyl-1H-indole-1-carboxylate (250 mg, $1 \mathrm{mmol}$ ). Purified by silica gel column chromatography (Hexanes/Ethyl Acetate 25:1, 20:1, $15: 1,10: 1,8: 1$ ) to give the product ( $480 \mathrm{mg}, 86 \%$ yield, $99: 1 \mathrm{er}, 10: 1 \mathrm{dr}$ ) as a white solid (melting point $\left.=56-60{ }^{\circ} \mathrm{C}\right)$.

Diacel CHIRALPAK IC Column: $98: 2 \mathrm{CO}_{2}: \mathrm{PrOH} ; 1.0 \mathrm{~mL} / \mathrm{min} ; 210 \mathrm{~nm}$.

Enantioenriched material: 99:1

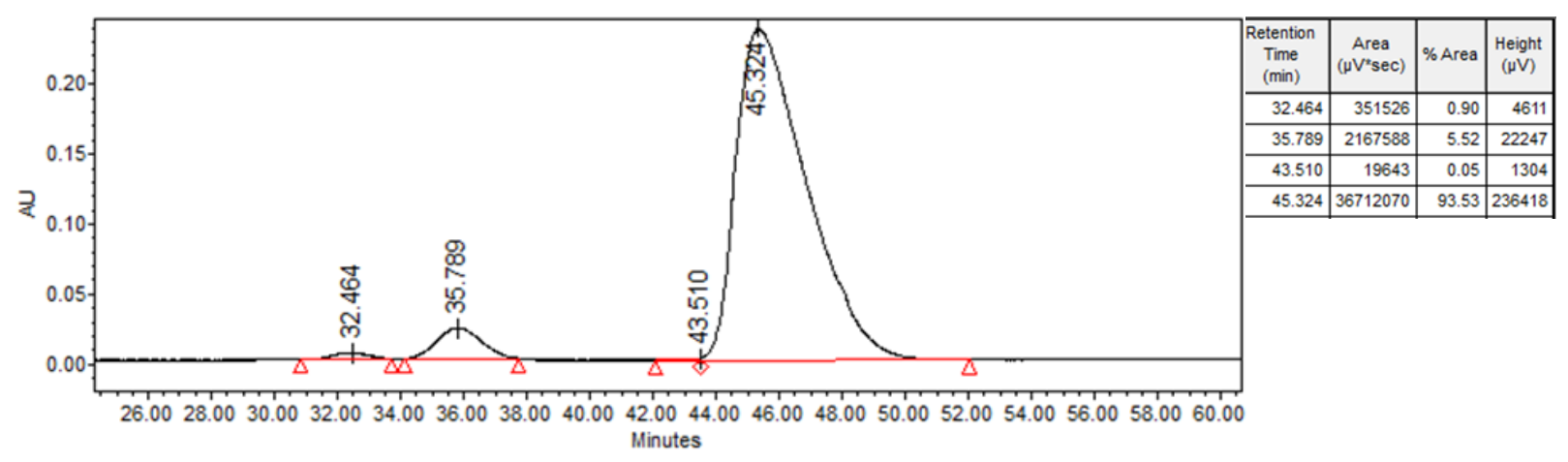

(1S,2S)-1-(benzofuran-2-yl)-2-methyl-2-((E)-2-(4,4,5,5-tetramethyl-1,3,2-dioxaborolan-2-yl)vinyl)hexan-1ol (3o)

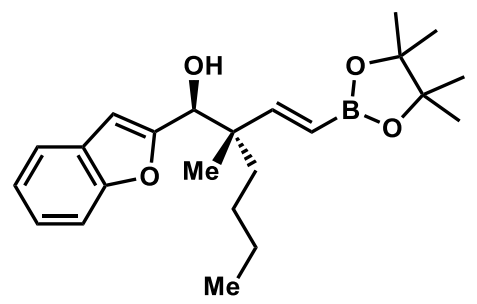

(30)

Prepared according to General Method III. Purified by silica gel column chromatography (Hexanes/Ethyl Acetate 25:1, 20:1, 15:1, 10:1, 8:1, 5:1) to give the product (19.1 $\mathrm{mg}$, 99\% yield, 98.5:1.5 er, 5:1 dr) as a colorless oil. ${ }^{1} \mathbf{H}$ NMR $\left(600 \mathrm{MHz}, \mathrm{CDCl}_{3}\right) \delta 7.56-7.52(\mathrm{~m}, 1 \mathrm{H}), 7.47-7.42$ $(\mathrm{m}, 1 \mathrm{H}), 7.28-7.18(\mathrm{~m}, 3 \mathrm{H}), 6.70-6.64(\mathrm{~d}, 18.4 \mathrm{~Hz}, 1 \mathrm{H}), 6.63(\mathrm{~s}, 1 \mathrm{H}), 5.61-5.54(\mathrm{~d}, 18.4 \mathrm{~Hz}, 1 \mathrm{H})$, $4.65(\mathrm{~s}, 1 \mathrm{H}), 2.16(\mathrm{~s}, 1 \mathrm{H}), 1.54-1.44(\mathrm{~m}, 2 \mathrm{H}), 1.30(\mathrm{~s}, 12 \mathrm{H}), 1.22-1.13(\mathrm{~m} 4 \mathrm{H}), 1.07(\mathrm{~s}, 3 \mathrm{H}), 0.88-$ 
$0.82(\mathrm{t}, 7.2 \mathrm{~Hz}, 3 \mathrm{H}) .{ }^{13} \mathrm{C}$ NMR $\left(151 \mathrm{MHz}, \mathrm{CDCl}_{3}\right) \delta$ 158.3, 157.3, 154.6, 128.2, 124.0, 122.8, 121.0, $111.3,104.8,83.4,74.4,47.2,37.0,26.3,25.0,24.9,23.5,17.2,14.2$. HRMS (m/z) calc. for $\left[\mathrm{M}-\mathrm{H}_{2} \mathrm{O}^{+}\right]$ $\mathrm{C}_{23} \mathrm{H}_{32} \mathrm{BO}_{3}{ }^{+}$: 367.2439 found: 367.2441. IR ( $\left.\mathrm{cm}^{-1}\right) 3447(\mathrm{w}), 2976(\mathrm{~m}), 2931(\mathrm{~m}), 2872(\mathrm{w}), 2360(\mathrm{w})$, $1633(\mathrm{~m}), 1455(\mathrm{~m}), 1351(\mathrm{~s}), 1146(\mathrm{~s}), 969(\mathrm{w}), 742(\mathrm{w}) .[\alpha]_{\mathrm{D}^{23}}=-26.2\left(\mathrm{c}=0.96, \mathrm{CH}_{2} \mathrm{Cl}_{2}, 1=1 \mathrm{dm}\right)$.

Diacel CHIRALPAK IE Column: $98: 2$ hexanes:iPrOH; $0.350 \mathrm{~mL} / \mathrm{min} ; 210 \mathrm{~nm}$.

Racemic material:

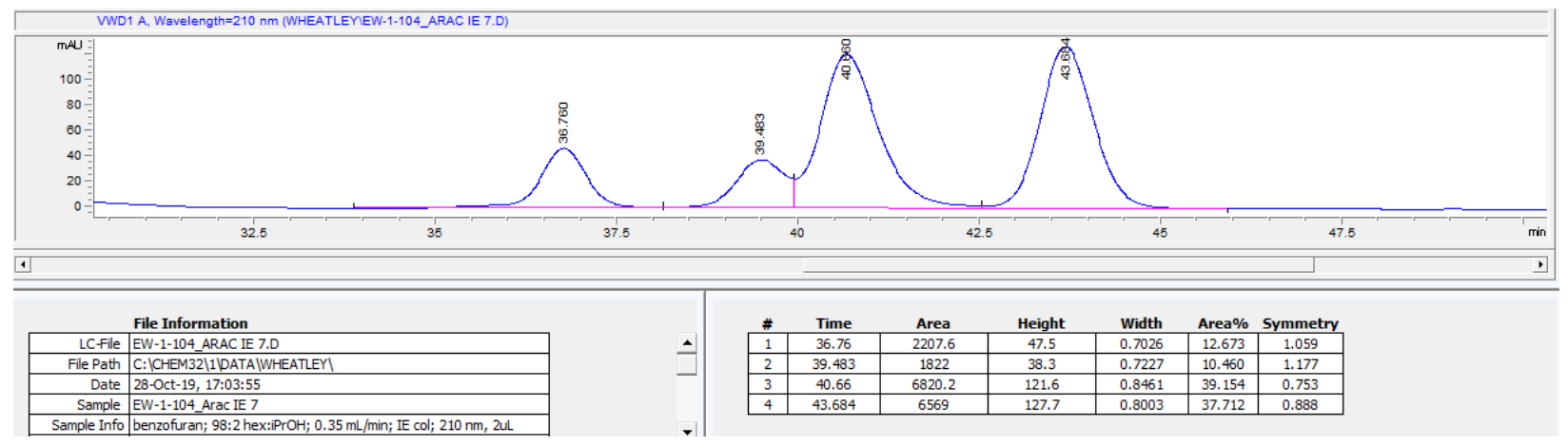

Enantioenriched material: 98.5:1.5 er

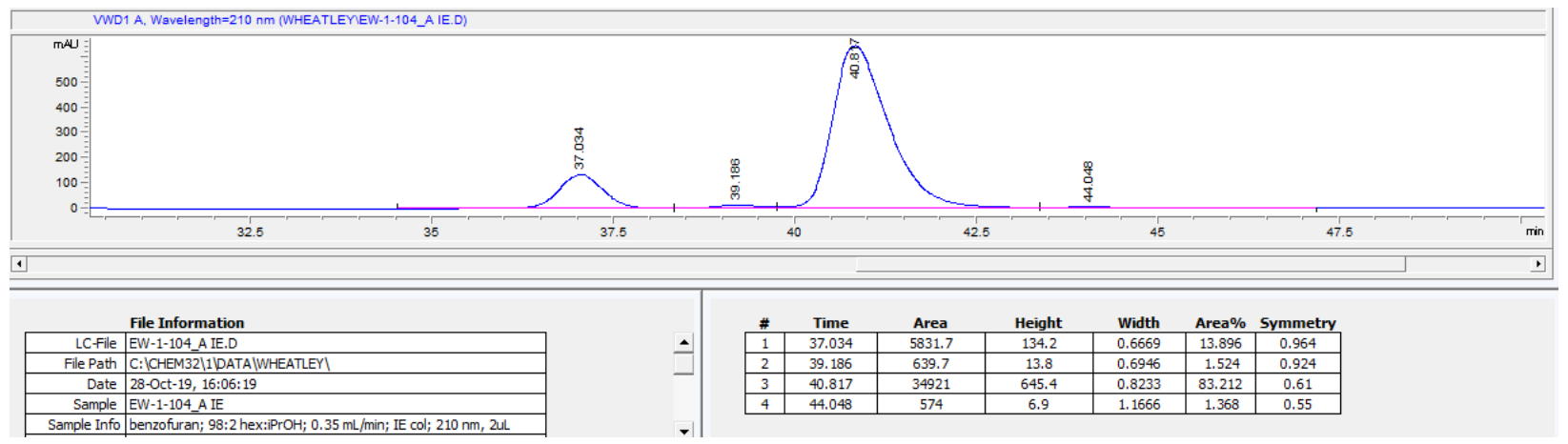

(1S,2S)-1-(benzo[b]thiophen-2-yl)-2-methyl-2-((E)-2-(4,4,5,5-tetramethyl-1,3,2-dioxaborolan-2yl)vinyl)hexan-1-ol (3p)

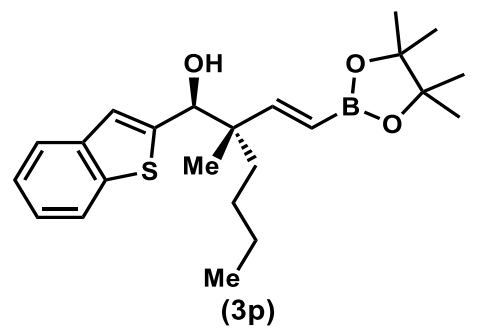

Prepared according to General Method III. Purified by silica gel column chromatography (Hexanes/Ethyl Acetate 25:1, 20:1, 15:1, 10:1, 8:1) to give the product (18.5 mg, 92\% yield, >99:1 er, 9:1 dr) as a colorless oil. ${ }^{1} \mathbf{H}$ NMR $\left(600 \mathrm{MHz}, \mathrm{CDCl}_{3}\right) \delta 7.82-7.78(\mathrm{~m}, 1 \mathrm{H}), 7.75-7.70(\mathrm{~m}, 1 \mathrm{H}), 7.36$ - $7.24(\mathrm{~m}, 3 \mathrm{H}), 7.21-7.16(\mathrm{~m}, 1 \mathrm{H}), 6.74-6.66(\mathrm{~d}, 18.5 \mathrm{~Hz}, 1 \mathrm{H}), 5.64-5.56(\mathrm{~d}, 18.4 \mathrm{~Hz}, 1 \mathrm{H}), 4.82(\mathrm{~s}$, 
$1 \mathrm{H}), 2.21(\mathrm{~s}, 1 \mathrm{H}), 1.53-1.46(\mathrm{~m}, 1 \mathrm{H}), 1.45-1.37(\mathrm{~m}, 1 \mathrm{H}), 1.30(\mathrm{~s}, 12 \mathrm{H}), 1.29-1.23(\mathrm{~m}, 2 \mathrm{H}), 1.20-$ $1.14(\mathrm{~m}, 2 \mathrm{H}), 1.06(\mathrm{~s}, 3 \mathrm{H}), 0.90-0.82(\mathrm{t}, 7.0 \mathrm{~Hz}, 3 \mathrm{H}) .{ }^{13} \mathrm{C} \mathrm{NMR}\left(151 \mathrm{MHz}, \mathrm{CDCl}_{3}\right) \delta$ 158.6, 145.0, $139.7,139.2,124.2,124.1,123.4,122.5,122.3,83.5,47.5,37.4,26.4,25.0,25.0,23.5,16.8,14.2$. HRMS (m/z) calc. for $\left[\mathrm{M}-\mathrm{H}_{2} \mathrm{O}^{+}\right] \mathrm{C}_{23} \mathrm{H}_{32} \mathrm{BO}_{2} \mathrm{~S}^{+}: 383.2211$ found: 383.2213. IR $\left(\mathrm{cm}^{-1}\right) 3450(\mathrm{w}), 2976(\mathrm{~m}), 2930$ (m), $2860(\mathrm{w}), 1633(\mathrm{~m}), 1379(\mathrm{~m}), 1351(\mathrm{~s}), 1145(\mathrm{~s}), 970(\mathrm{w}), 851(\mathrm{w}) .[\alpha]_{\mathrm{D}^{23}}=-10.1(\mathrm{c}=0.93$, $\left.\mathrm{CH}_{2} \mathrm{Cl}_{2}, \mathrm{l}=1 \mathrm{dm}\right)$.

Phenomenex lux Amylose 1-AD Column: 95:5 $\mathrm{CO}_{2}: \mathrm{iPrOH} ; 1.0 \mathrm{~mL} / \mathrm{min} ; 210 \mathrm{~nm}$.

Racemic material:

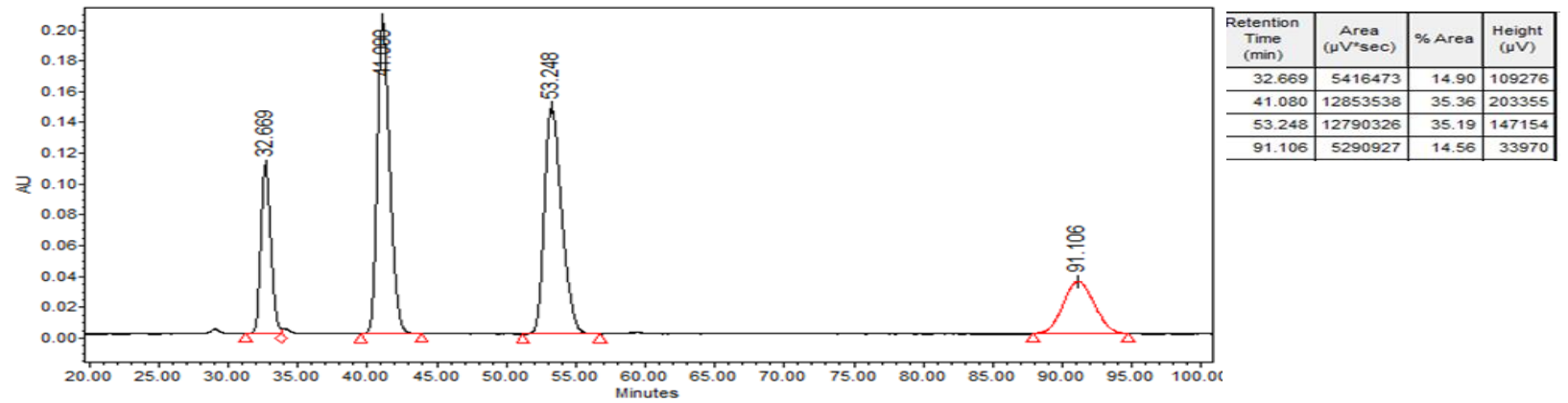

Enantioenriched material: >99:1 er

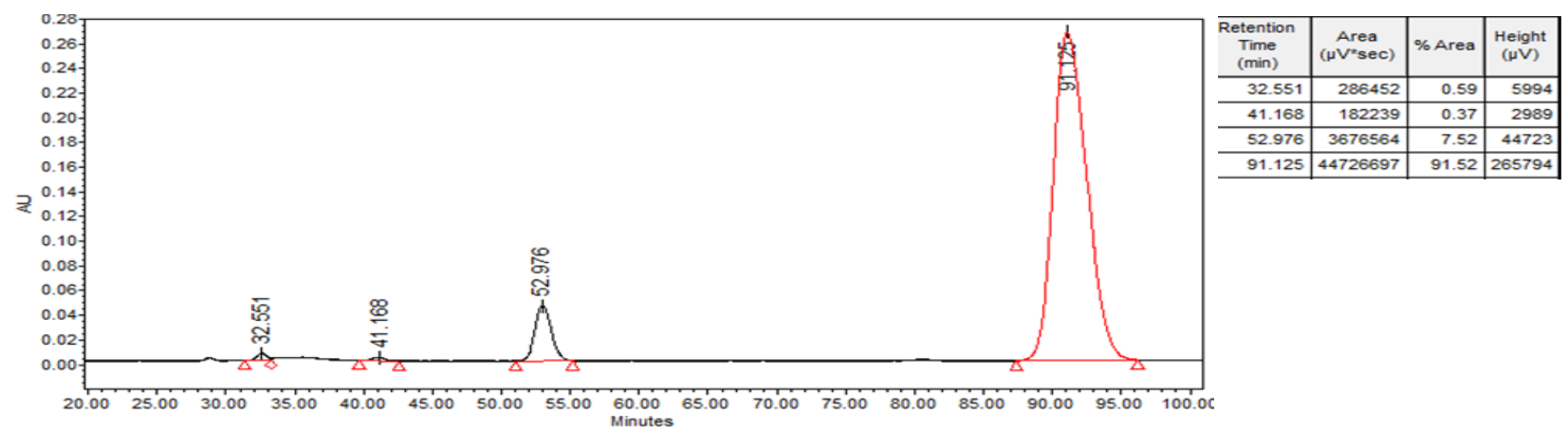

(3R,4S,E)-2,4-dimethyl-1-phenyl-4-((E)-2-(4,4,5,5-tetramethyl-1,3,2-dioxaborolan-2-yl)vinyl)oct-1-en-3-ol (5a)

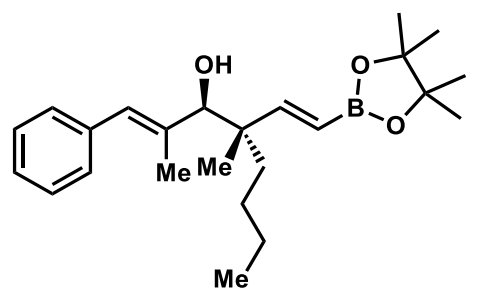

(5a)

Prepared according to General Method III. Purified by silica gel column chromatography (Hexanes/Ethyl Acetate 25:1, 20:1, 15:1, 10:1) to give the product (18.8 mg, 97\% yield, >99:1 er, 4:1 dr) as a colorless oil.

\section{Major Diastereomer}




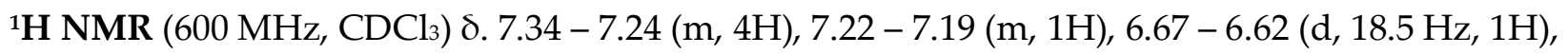
$6.43(\mathrm{~s}, 1 \mathrm{H}), 5.49-5.43(\mathrm{~d}, 18.5 \mathrm{~Hz}, 1 \mathrm{H}), 4.00(\mathrm{~s}, 1 \mathrm{H}), 1.83(\mathrm{~s}, 3 \mathrm{H}), 1.78-1.65(\mathrm{~m}, 2 \mathrm{H}), 1.57-1.44$ $(\mathrm{m}, 2 \mathrm{H}), 1.28-1.24(\mathrm{~d}, 8.1 \mathrm{~Hz}, 12 \mathrm{H}), 1.23-1.15(\mathrm{~m}, 2 \mathrm{H}), 1.10(\mathrm{~s}, 3 \mathrm{H}), 0.90-0.85(\mathrm{t}, 7.2 \mathrm{~Hz}, 3 \mathrm{H}) .{ }^{13} \mathrm{C}$ NMR (151 MHz, $\left.\mathrm{CDCl}_{3}\right) \delta$ 159.2, 138.5, 137.7, 129.2, 128.5, 128.2, 126.5, 83.9, 83.3, 47.2, 37.5, 26.6, 25.0, 24.8, 23.7, 19.0, 16.6.

\section{Minor Diastereomer}

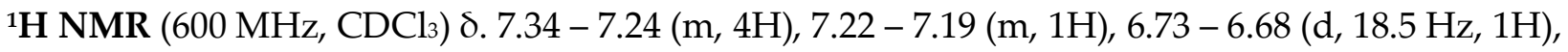
$6.43(\mathrm{~s}, 1 \mathrm{H}), 5.52-5.48(\mathrm{~d}, 18.9 \mathrm{~Hz}, 1 \mathrm{H}), 4.00(\mathrm{~s}, 1 \mathrm{H}), 1.87(\mathrm{~s}, 3 \mathrm{H}), 1.78-1.65(\mathrm{~m}, 2 \mathrm{H}), 1.57-1.44$ $(\mathrm{m}, 2 \mathrm{H}), 1.28-1.24(\mathrm{~d}, 8.1 \mathrm{~Hz}, 12 \mathrm{H}), 1.23-1.15(\mathrm{~m}, 2 \mathrm{H}), 1.03(\mathrm{~s}, 3 \mathrm{H}), 0.90-0.85(\mathrm{t}, 7.2 \mathrm{~Hz}, 3 \mathrm{H}) .{ }^{13} \mathrm{C}$ NMR (151 MHz, $\left.\mathrm{CDCl}_{3}\right) \delta$ 159.8, 138.0, 137.7, 129.2, 129.1, 128.2, 126.5, 83.7, 83.3, 47.3, 37.7, 26.5, 25.0, 24.9, 23.6, 18.1, 16.1.

HRMS (m/z) calc. for [M-H2 $\left.\mathrm{O}^{+}\right] \mathrm{C}_{24} \mathrm{H}_{36} \mathrm{BO}_{2}{ }^{+}: 367.2803$ found: 367.2817. IR (cm-1) $3446(\mathrm{~s}), 2976(\mathrm{~m})$, $2931(\mathrm{~m}), 1633(\mathrm{~m}), 1351(\mathrm{~s}), 1146(\mathrm{~s}), 850(\mathrm{w}), 700(\mathrm{w}) .[\alpha]_{\mathrm{D}^{23}}=-21.5\left(\mathrm{c}=0.94, \mathrm{CH}_{2} \mathrm{Cl}_{2}, 1=1 \mathrm{dm}\right)$.

Phenomenex lux Amylose 1-AD Column: 98:2 $\mathrm{CO}_{2}: \mathrm{iPrOH} ; 1.0 \mathrm{~mL} / \mathrm{min} ; 210 \mathrm{~nm}$.

\section{Racemic material:}

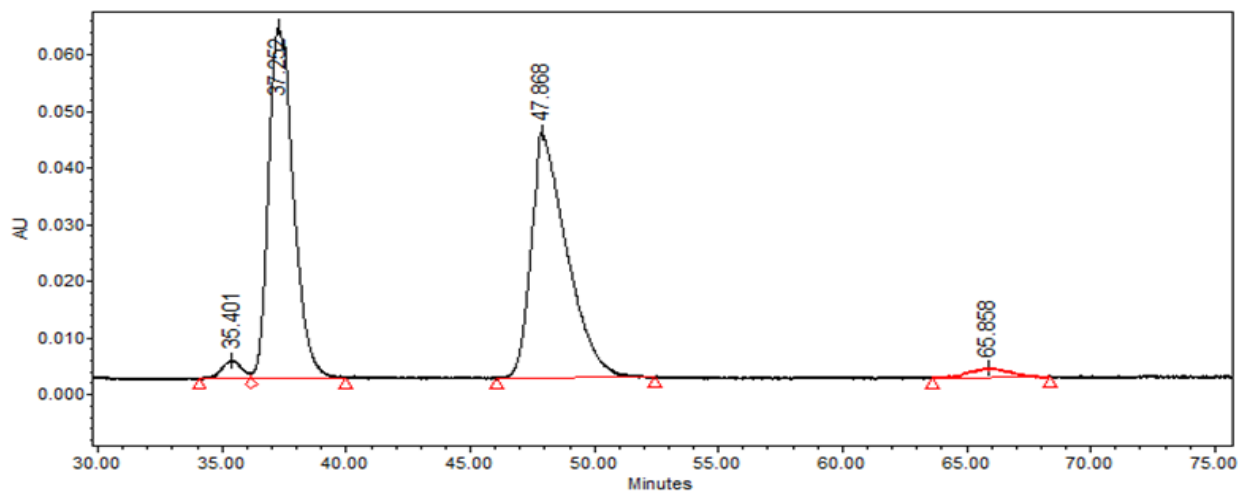

\begin{tabular}{c|r|r|r|}
\hline $\begin{array}{c}\text { Retention } \\
\text { Time } \\
(\mathrm{min})\end{array}$ & $\begin{array}{c}\text { Area } \\
\left(\mu \mathrm{V}^{*} \mathrm{sec}\right)\end{array}$ & $\%$ Area & $\begin{array}{c}\text { Height } \\
(\mu \mathrm{V})\end{array}$ \\
\hline 35.401 & 175053 & 1.95 & 3236 \\
\hline 37.252 & 4319841 & 48.09 & 61932 \\
\hline 47.868 & 4313871 & 48.02 & 43494 \\
\hline 65.858 & 173883 & 1.94 & 1709 \\
\hline
\end{tabular}

Enantioenriched material:

Major: >99:1 er

Minor: 97:3 er

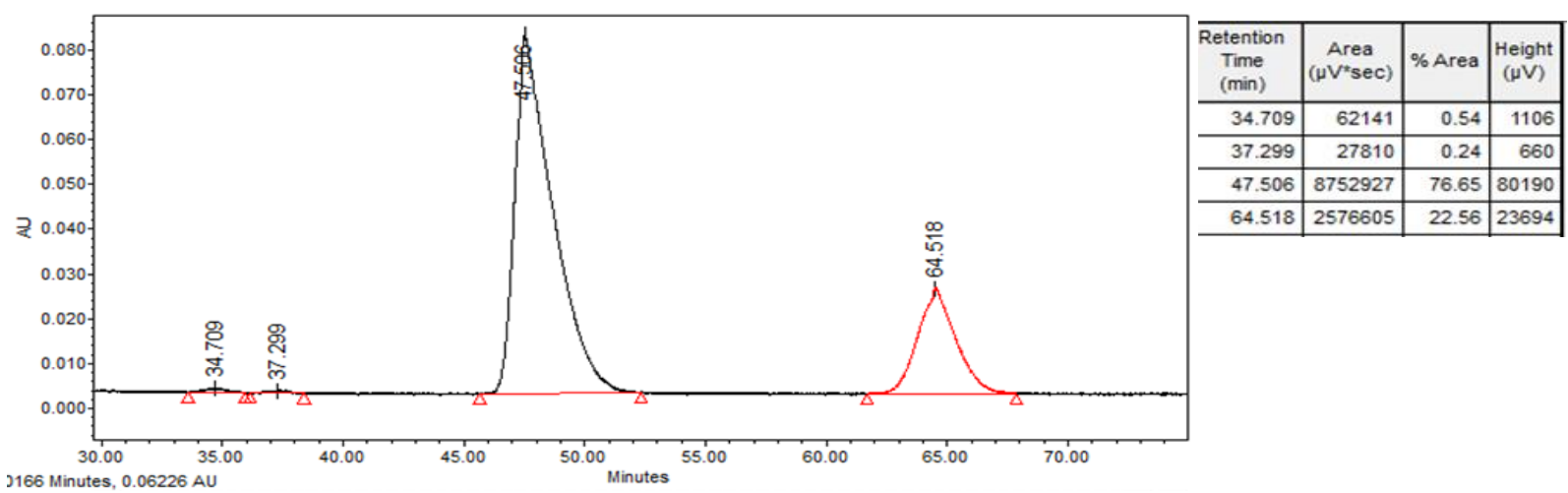

(4R,5S,E)-3,5-dimethyl-5-((E)-2-(4,4,5,5-tetramethyl-1,3,2-dioxaborolan-2-yl)vinyl)non-2-en-4-ol (5b) 


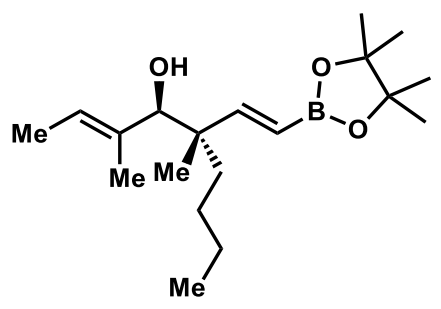

(5b)

Prepared according to General Method III. Purified by silica gel column chromatography (Hexanes/Ethyl Acetate 25:1, 20:1, 15:1, 10:1, 8:1) to give the product (15.8 mg, 99\% yield, 99:1 er, 6:1 dr) as a colorless oil. ${ }^{1} \mathrm{H}$ NMR $\left(600 \mathrm{MHz}, \mathrm{CDCl}_{3}\right) \delta 6.62-6.55(\mathrm{~d}, 18.4 \mathrm{~Hz}, 1 \mathrm{H}), 5.46-5.36(\mathrm{~m}$, 2H), $3.83(\mathrm{~s}, 1 \mathrm{H}), 1.66(\mathrm{~s}, 1 \mathrm{H}), 1.64-1.59(\mathrm{~d}, 6.7 \mathrm{~Hz}, 3 \mathrm{H}), 1.58(\mathrm{~s}, 3 \mathrm{H}), 1.50-1.34(\mathrm{~m}, 3 \mathrm{H}), 1.31-$ $1.26(\mathrm{~d}, 4.3 \mathrm{~Hz}, 12 \mathrm{H}), 1.21-1.12(\mathrm{~m}, 3 \mathrm{H}), 1.03(\mathrm{~s}, 3 \mathrm{H}), 0.92-0.86(\mathrm{t}, 7.1 \mathrm{~Hz}, 3 \mathrm{H}) .{ }^{13} \mathrm{C}$ NMR (151 $\left.\mathrm{MHz}_{2} \mathrm{CDCl}_{3}\right) \delta$ 159.5, 136.0, 123.2, 83.7, 83.2, 46.8, 37.2, 26.6, 25.1, 24.7, 23.7, 19.1, 14.4, 14.3, 13.3. HRMS (m/z) calc. for [M- $\left.\mathrm{H}_{2} \mathrm{O}^{+}\right] \mathrm{C}_{19} \mathrm{H}_{34} \mathrm{BO}_{2}{ }^{+}: 305.2646$ found: 305.2650. IR (cm $\left.{ }^{-1}\right) 3488(\mathrm{w}), 2977(\mathrm{~m})$, $2931(\mathrm{~m}), 2861(\mathrm{w}), 1633(\mathrm{~m}), 1458(\mathrm{w}), 1372(\mathrm{~m}), 1351(\mathrm{~s}), 1147(\mathrm{~s}), 970(\mathrm{w}), 851(\mathrm{w}) .[\alpha]_{\mathrm{D}^{23}}=-8.7$ $\left(\mathrm{c}=0.79, \mathrm{CH}_{2} \mathrm{Cl}_{2}, \mathrm{l}=1 \mathrm{dm}\right)$.

Phenomenex lux Amylose 1-AD Column: 95:5 $\mathrm{CO}_{2}: \mathrm{iPrOH} ; 1.0 \mathrm{~mL} / \mathrm{min} ; 210 \mathrm{~nm}$.

Racemic material:

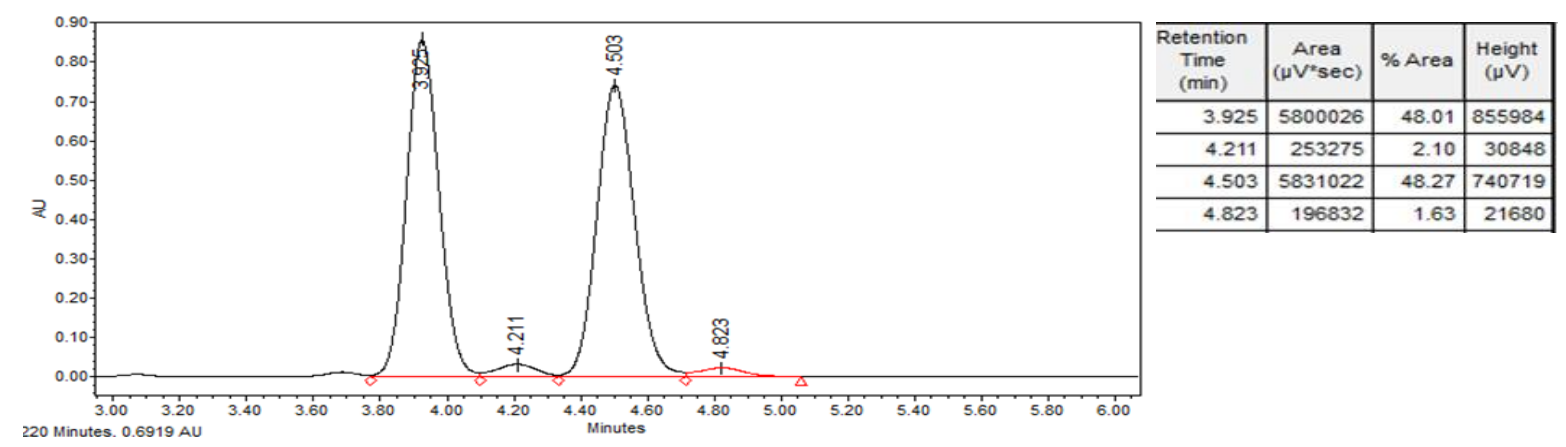

\section{Enantioenriched material: 99:1 er}
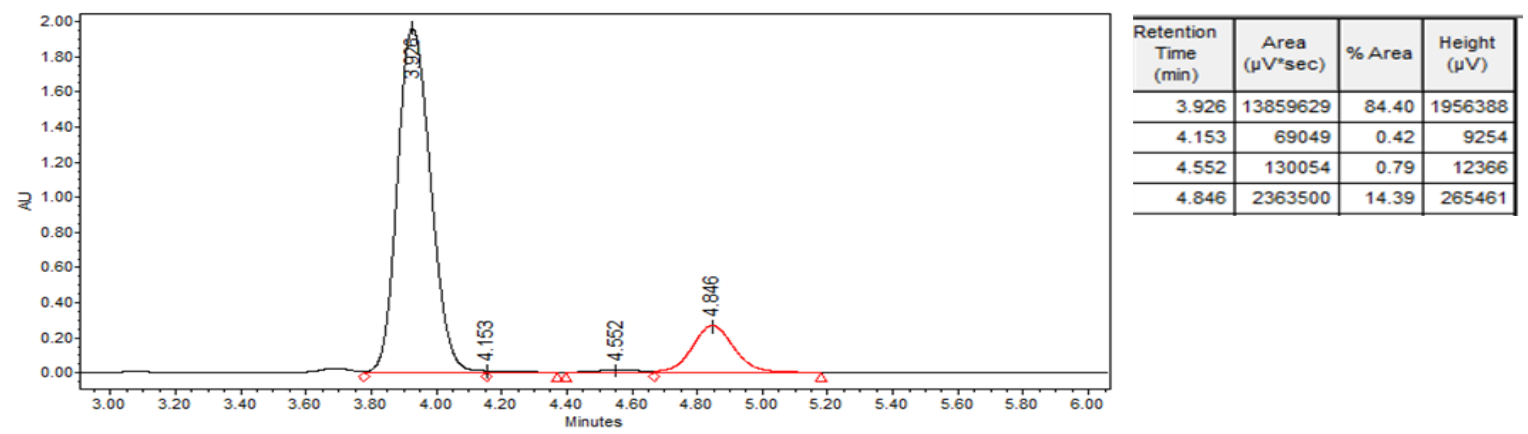

(1R,2S)-1-(cyclohex-1-en-1-yl)-2-methyl-2-((E)-2-(4,4,5,5-tetramethyl-1,3,2-dioxaborolan-2-yl)vinyl)hexan$1-$ ol $(5 c)$ 


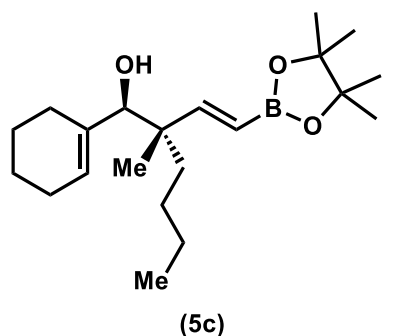

Prepared according to General Method III. Purified by silica gel column chromatography (Hexanes/Ethyl Acetate 25:1, 20:1, 15:1, 10:1, 8:1) to give the product (16.7 mg, 95\% yield, >99:1 er, 6:1 dr) as a colorless oil.

\section{Major Diastereomer}

${ }^{1} \mathbf{H}$ NMR $\left(600 \mathrm{MHz}, \mathrm{CDCl}_{3}\right) \delta 6.61-6.55(\mathrm{~d}, 18.5 \mathrm{~Hz}, 1 \mathrm{H}), 5.61-5.57(\mathrm{~m}, 1 \mathrm{H}), 5.43-5.37(\mathrm{~d}, 18.7$ $\mathrm{Hz}, 1 \mathrm{H}), 3.74(\mathrm{~s}, 1 \mathrm{H}), 2.06-2.01(\mathrm{~m}, 2 \mathrm{H}), 1.99-1.95(\mathrm{~m}, 1 \mathrm{H}), 1.90-1.84(\mathrm{~m}, 1 \mathrm{H}), 1.63$ - 1.57 (m, 2H), $1.52-1.46(\mathrm{~m}, 2 \mathrm{H}), 1.45-1.35(\mathrm{~m}, 2 \mathrm{H}), 1.29-1.25(\mathrm{~d}, 6.9 \mathrm{~Hz}, 12 \mathrm{H}), 1.23-1.19(\mathrm{~m}, 2 \mathrm{H}), 1.17$ $1.11(\mathrm{~m}, 2 \mathrm{H}), 1.01(\mathrm{~s}, 3 \mathrm{H}), 0.89-0.84(\mathrm{t}, 7.3 \mathrm{~Hz}, 3 \mathrm{H}) .{ }^{13} \mathrm{C}$ NMR $\left(151 \mathrm{MHz}, \mathrm{CDCl}_{3}\right) \delta$ 159.5, 138.5, $125.4,83.2,82.6,46.8,37.2,27.3,26.6,25.2,25.0,24.7,23.7,22.9,22.7,18.9$.

\section{Minor Diastereomer}

${ }^{1} \mathrm{H}$ NMR $\left(600 \mathrm{MHz}, \mathrm{CDCl}_{3}\right) \delta 6.65$ - 6.61 (d, $\left.18.4 \mathrm{~Hz}, 1 \mathrm{H}\right), 5.61$ - 5.57 (m, 1H), 5.46 - 5.41 (d, 18.5 $\mathrm{Hz}, 1 \mathrm{H}), 3.74(\mathrm{~s}, 1 \mathrm{H}), 2.06-2.01(\mathrm{~m}, 2 \mathrm{H}), 1.99-1.95(\mathrm{~m}, 1 \mathrm{H}), 1.90-1.84(\mathrm{~m}, 1 \mathrm{H}), 1.63$ - $1.57(\mathrm{~m}$, 2H), 1.52 - $1.46(\mathrm{~m}, 2 \mathrm{H}), 1.45-1.35(\mathrm{~m}, 2 \mathrm{H}), 1.29-1.25(\mathrm{~d}, 6.9 \mathrm{~Hz}, 12 \mathrm{H}), 1.23-1.19(\mathrm{~m}, 2 \mathrm{H}), 1.17$ $1.11(\mathrm{~m}, 2 \mathrm{H}), 0.95(\mathrm{~s}, 3 \mathrm{H}), 0.89-0.84(\mathrm{t}, 7.3 \mathrm{~Hz}, 3 \mathrm{H}) .{ }^{13} \mathrm{C}$ NMR $\left(151 \mathrm{MHz}, \mathrm{CDCl}_{3}\right) \delta$ 160.2, 137.8, $126.3,83.3,82.4,47.0,37.6,26.7,26.5,25.3,25.0,24.9,23.7,23.0,22.7,17.8$.

HRMS (m/z) calc. for [M- $\left.\mathrm{H}_{2} \mathrm{O}^{+}\right] \mathrm{C}_{21} \mathrm{H}_{36} \mathrm{BO}_{2}{ }^{+}: 331.2803$ found: 331.2817 . IR (cm-1) $3446(\mathrm{~s}), 2930(\mathrm{~m})$, $1632(\mathrm{~m}), 1371(\mathrm{~s}), 1146(\mathrm{~m}), 970(\mathrm{w}), 852(\mathrm{w}) .[\alpha]_{\mathrm{D}^{23}}=-12.9\left(\mathrm{c}=0.84, \mathrm{CH}_{2} \mathrm{Cl}_{2}, \mathrm{l}=1 \mathrm{dm}\right)$.

Diacel CHIRALPAK IC Column: $98: 2 \mathrm{CO}_{2}: \mathrm{iPrOH} ; 1.0 \mathrm{~mL} / \mathrm{min} ; 210 \mathrm{~nm}$.

Racemic material:
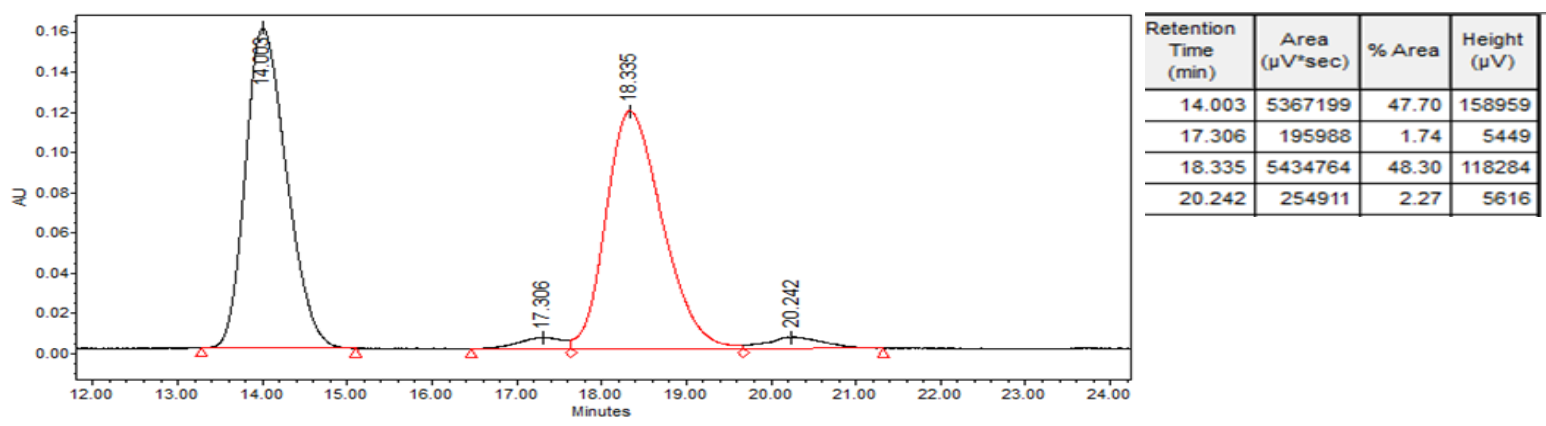

Enantioenriched material: >99:1 er 


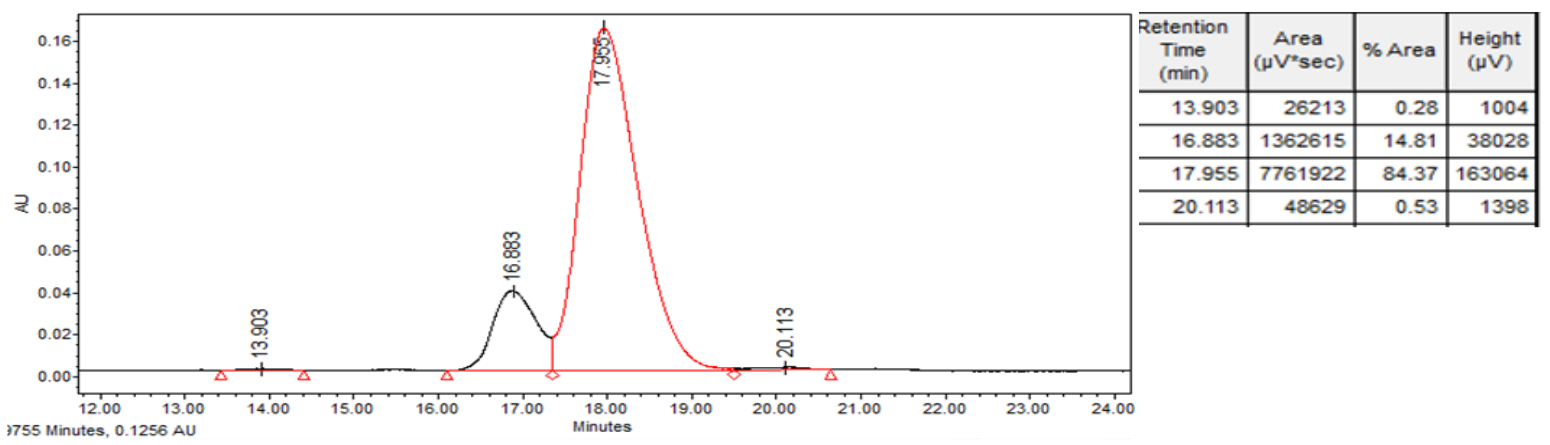

(1S,2S)-1-(6,6-dimethylbicyclo[3.1.1] hept-2-en-2-yl)-2-methyl-2-((E)-2-(4,4,5,5-tetramethyl-1,3,2dioxaborolan-2-yl)vinyl)hexan-1-ol (5d)

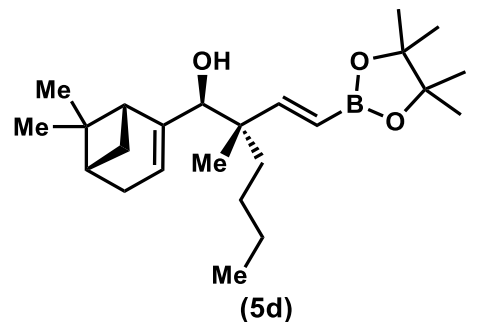

Prepared according to General Method III. Purified by silica gel column chromatography (Hexanes/Ethyl Acetate 25:1, 20:1, 15:1, 10:1) to give the product (15.9 mg, 82\% yield, 9:1 dr) as a colorless oil. ${ }^{1} \mathbf{H}$ NMR $\left(600 \mathrm{MHz}, \mathrm{CDCl}_{3}\right) \delta 6.62-6.55(\mathrm{~d}, 18.5 \mathrm{~Hz}, 1 \mathrm{H}), 5.45-5.39(\mathrm{~d}, 18.5 \mathrm{~Hz}, 1 \mathrm{H})$, $5.37(\mathrm{~s}, 1 \mathrm{H}), 3.82(\mathrm{~s}, 1 \mathrm{H}), 2.35-2.20(\mathrm{~m}, 4 \mathrm{H}), 2.05(\mathrm{~s}, 1 \mathrm{H}), 1.40-1.34(\mathrm{~m}, 2 \mathrm{H}), 1.28-1.26(\mathrm{~d}, 2.5 \mathrm{~Hz}$, 12H), $1.24(\mathrm{~s}, 3 \mathrm{H}), 1.22-1.04(\mathrm{~m}, 6 \mathrm{H}), 0.92(\mathrm{~s}, 3 \mathrm{H}), 0.88-0.85(\mathrm{t}, 7.5 \mathrm{~Hz}, 3 \mathrm{H}), 0.84(\mathrm{~s}, 3 \mathrm{H}) .{ }^{13} \mathrm{C} \mathrm{NMR}$ (151 MHz, $\left.\mathrm{CDCl}_{3}\right) \delta$ 160.0, 147.1, 121.7, 83.2, 81.3, 47.0, 42.9, 40.5, 37.3, 37.2, 31.8, 31.6, 26.4, 26.3,

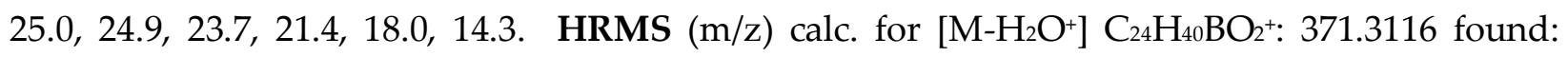
371.3127. IR (cm-1) $3502(\mathrm{w}), 2978(\mathrm{~m}), 2932(\mathrm{~m}), 1633(\mathrm{~m}), 1351(\mathrm{~s}), 1146(\mathrm{~s}), 970(\mathrm{w}), 850(\mathrm{w}) .[\alpha]_{\mathrm{D}^{23}}$ $=-22.9\left(\mathrm{c}=0.80, \mathrm{CH}_{2} \mathrm{Cl}_{2}, \mathrm{l}=1 \mathrm{dm}\right)$.

(3S,4S)-4-methyl-1-phenyl-4-((E)-2-(4,4,5,5-tetramethyl-1,3,2-dioxaborolan-2-yl)vinyl)oct-1-yn-3-ol (5e)

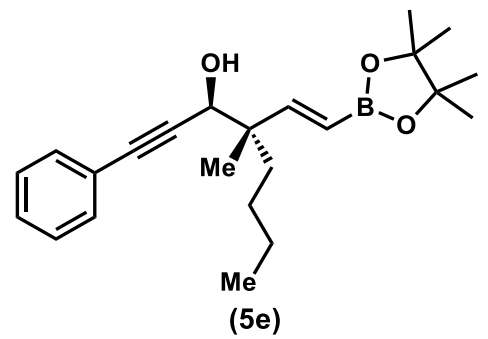

Prepared according to General Method III. Purified by silica gel column chromatography (Hexanes/Ethyl Acetate 25:1, 20:1, 15:1, 10:1) to give the product (15.4 mg, 84\% yield, 98.5:1.5 er, 2:1 dr) as a colorless oil. 


\section{Major Diastereomer}

${ }^{1}$ H NMR (600 MHz, $\left.\mathrm{CDCl}_{3}\right) \delta 7.33$ - 7.27 (m, 5H), $6.64-6.58(\mathrm{~d}, 18.5 \mathrm{~Hz}, 1 \mathrm{H}), 5.61$ - 5.54 (d, 18.4 $\mathrm{Hz}, 1 \mathrm{H}), 4.41(\mathrm{~s}, 1 \mathrm{H}), 1.89(\mathrm{~s}, 1 \mathrm{H}), 1.59-1.52(\mathrm{~m}, 2 \mathrm{H}), 1.30$ - 1.26 (d, $3.6 \mathrm{~Hz} 12 \mathrm{H}), 1.24$ - 1.20 (m, $4 \mathrm{H}), 1.17$ (s, 3H), $0.91-0.85$ (t, $7.2 \mathrm{~Hz}, 3 \mathrm{H}) .{ }^{13} \mathrm{C}$ NMR $\left(151 \mathrm{MHz}, \mathrm{CDCl}_{3}\right) \delta 157.6,131.9,128.4,128.4$, $122.9,88.0,86.4,83.4,69.7,47.3,36.9,26.2,25.0,24.9,23.6,17.7,14.2$.

\section{Minor Diastereomer}

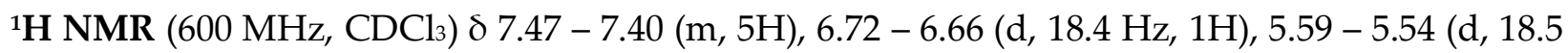
$\mathrm{Hz}, 1 \mathrm{H}), 4.34(\mathrm{~s}, 1 \mathrm{H}), 1.83(\mathrm{~s}, 1 \mathrm{H}), 1.68-1.61(\mathrm{~m}, 2 \mathrm{H}), 1.30$ - $1.26(\mathrm{~d}, 3.6 \mathrm{~Hz} 12 \mathrm{H}), 1.24$ - $1.20(\mathrm{~m}$, $4 \mathrm{H}), 1.15$ (s, 3H), $0.91-0.85$ (t, $7.2 \mathrm{~Hz}, 3 \mathrm{H}) .{ }^{13} \mathrm{C}$ NMR $\left(151 \mathrm{MHz}, \mathrm{CDCl}_{3}\right) \delta 157.2,131.8,128.5,128.3$, 122., 88.4, 86.5, 83.4, 70.1, 47.0, 36.3, 26.3, 25.0, 25.0, 23.6, 18.8, 14.2.

HRMS (m/z) calc. for [M- $\left.\mathrm{H}_{2} \mathrm{O}^{+}\right] \mathrm{C}_{23} \mathrm{H}_{32} \mathrm{BO}_{2}{ }^{+}: 351.2490$ found: 351.2492. IR ( $\left.\mathrm{cm}^{-1}\right) 2931(\mathrm{w}), 1634(\mathrm{~m})$, $1351(\mathrm{~s}), 1145(\mathrm{~m}), 970(\mathrm{w}), 851(\mathrm{w}) .[\alpha]_{\mathrm{D}^{23}}=-12.6\left(\mathrm{c}=0.77, \mathrm{CH}_{2} \mathrm{Cl}_{2}, 1=1 \mathrm{dm}\right)$.

Diacel CHIRALPAK IC Column: 96:4 $\mathrm{CO}_{2}: \mathrm{iPrOH} ; 1.0 \mathrm{~mL} / \mathrm{min} ; 230 \mathrm{~nm}$.

Racemic material:

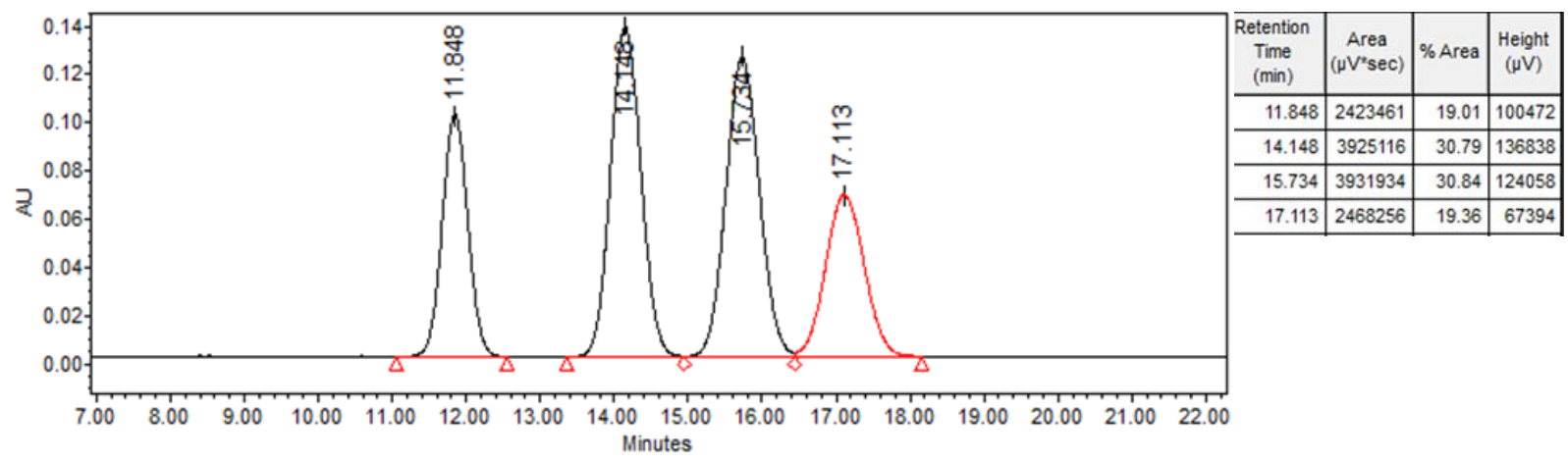

\section{Enantioenriched material:}

Major: 98.5:1.5 er

Minor: >99:1 er

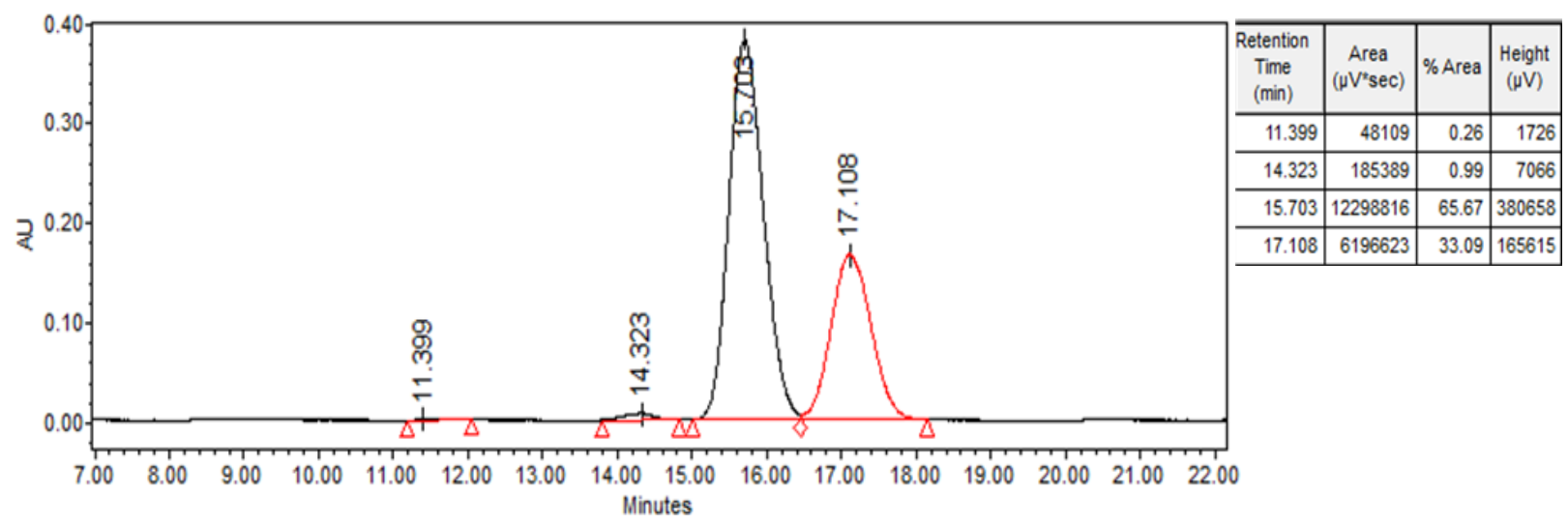

(3R,4S)-2,2,4-trimethyl-4-((E)-2-(4,4,5,5-tetramethyl-1,3,2-dioxaborolan-2-yl)vinyl)octan-3-ol (5f) 


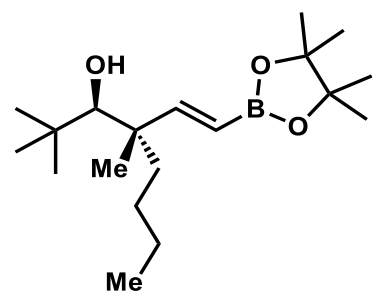

(5f)

Prepared according to General Method III. Purified by silica gel column chromatography (Hexanes/Ethyl Acetate 25:1, 20:1, 15:1, 10:1) to give the product (11.0 mg, 68 \% yield, 99:1 er, >20:1 $\mathrm{dr})$ as a colorless oil. ${ }^{1} \mathrm{H}$ NMR $\left(600 \mathrm{MHz}, \mathrm{CDCl}_{3}\right) \delta 6.72-6.62(\mathrm{~d}, 18.5 \mathrm{~Hz}, 1 \mathrm{H}), 5.42-5.32(\mathrm{~d}$, $18.5 \mathrm{~Hz}, 1 \mathrm{H}), 3.29-3.22(\mathrm{~d}, 6.2 \mathrm{~Hz}, 1 \mathrm{H}), 1.61(\mathrm{~s}, 1 \mathrm{H}), 1.57-1.53(\mathrm{~m}, 1 \mathrm{H}), 1.49-1.42(\mathrm{~m}, 1 \mathrm{H}), 1.29-$ $1.23(\mathrm{~d}, 5.5 \mathrm{~Hz}, 12 \mathrm{H}), 1.24-1.18(\mathrm{~m}, 2 \mathrm{H}), 1.16-1.08(\mathrm{~m}, 2 \mathrm{H}), 1.06(\mathrm{~s}, 3 \mathrm{H}), 0.94(\mathrm{~s}, 9 \mathrm{H}), 0.88-0.83$ (t, 7.2 Hz, 3H). ${ }^{13} \mathrm{C}$ NMR (151 MHz, $\left.\mathrm{CDCl}_{3}\right) \delta$ 161.6, 84.0, 83.1, 47.9, 40.7, 37.8, 28.8, 26.1, 25.1, 24.7, 23.6, 17.9, 14.3. HRMS (m/z) calc. for $\left[\mathrm{M}-\mathrm{H}_{2} \mathrm{O}^{+}\right] \mathrm{C}_{19} \mathrm{H}_{36} \mathrm{BO}_{2}{ }^{+}: 307.2803$ found: 307.2803. IR $\left(\mathrm{cm}^{-1}\right)$ $3522(\mathrm{w}), 2956(\mathrm{~s}), 2871(\mathrm{~m}), 1631(\mathrm{~m}), 1351(\mathrm{~s}), 1147(\mathrm{~s}), 970(\mathrm{~m}), 851(\mathrm{w}), 739(\mathrm{~m}) .[\alpha]_{\mathrm{D}^{23}}=-15.0$ (c $=0.55, \mathrm{CH}_{2} \mathrm{Cl}_{2}, 1=1 \mathrm{dm}$ ).

Phenomenex lux Amylose 1-AD Column: 97:3 $\mathrm{CO}_{2}: \mathrm{iPrOH} ; 1.0 \mathrm{~mL} / \mathrm{min} ; 210 \mathrm{~nm}$.

Racemic material:

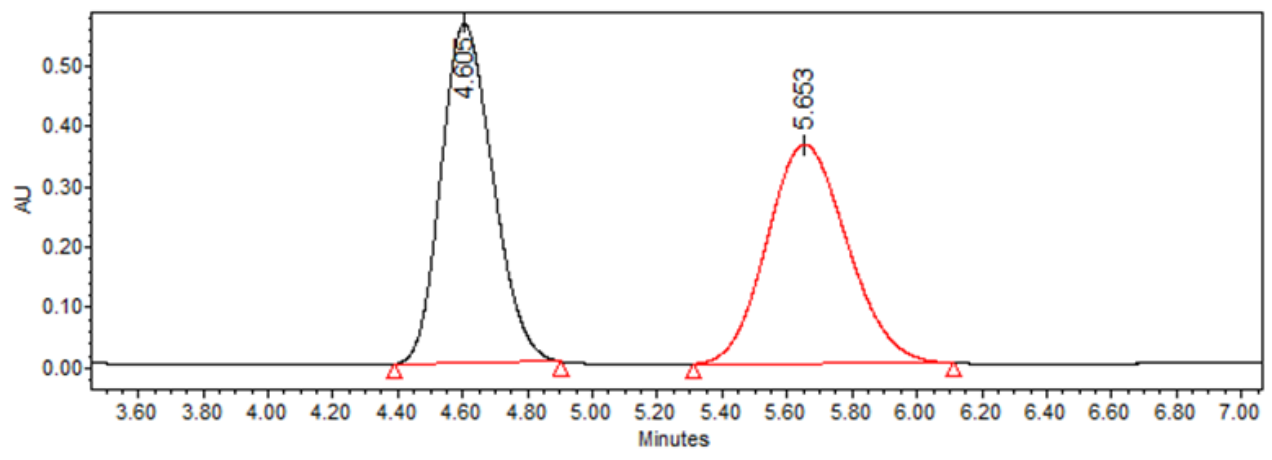

\begin{tabular}{c|c|c|c|}
\hline $\begin{array}{c}\text { Retention } \\
\text { Time } \\
(\mathrm{min})\end{array}$ & $\begin{array}{c}\text { Area } \\
\left(\mu \mathrm{V}^{*} \mathrm{sec}\right)\end{array}$ & $\%$ Area & $\begin{array}{c}\text { Height } \\
(\mu \mathrm{V})\end{array}$ \\
\hline 4.605 & 6253235 & 50.06 & 561247 \\
\hline 5.653 & 6239466 & 49.94 & 362596 \\
\hline
\end{tabular}

Enantioenriched material: 99:1 er

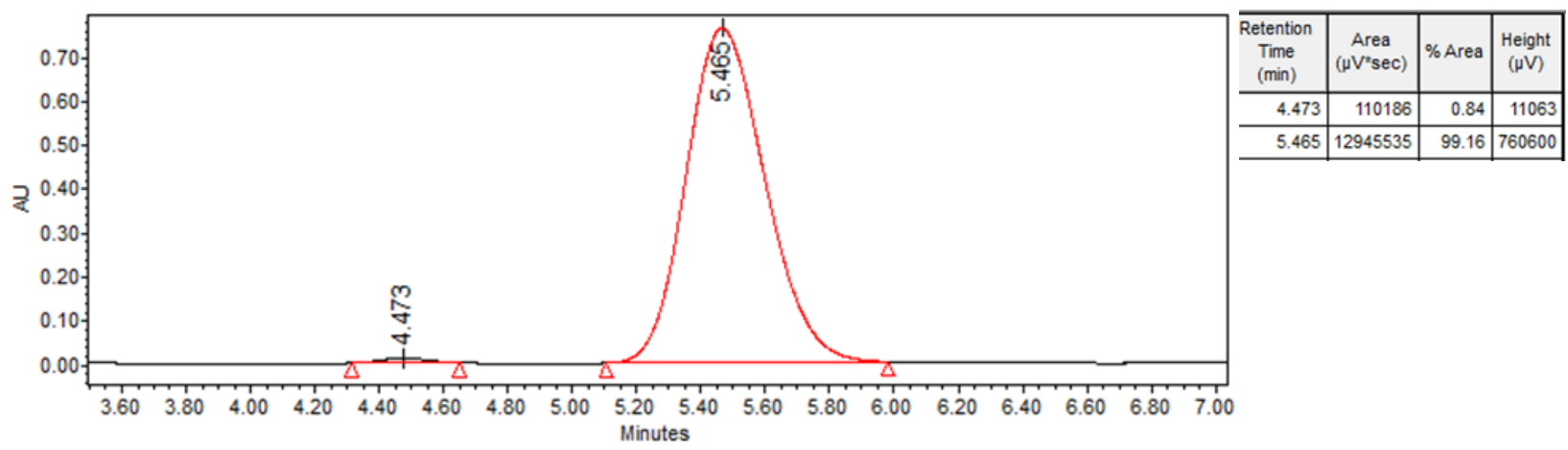

(1R,2S)-1-cyclohexyl-2-methyl-2-((E)-2-(4,4,5,5-tetramethyl-1,3,2-dioxaborolan-2-yl)vinyl)hexan-1-ol (5g) 


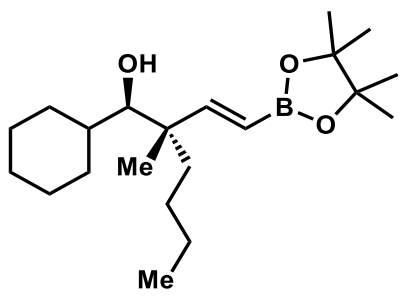

(5g)

Prepared according to General Method III. Purified by silica gel column chromatography (Hexanes/Ethyl Acetate 25:1, 20:1, 15:1, 10:1, 8:1) to give the product (17.7 mg, 98\% yield, 99:1 er, 10:1 dr) as a colorless oil. ${ }^{1} \mathrm{H}$ NMR $\left(600 \mathrm{MHz} \mathrm{CDCl}_{3}\right) \delta 6.60-6.54(\mathrm{~d}, 18.5 \mathrm{~Hz}, 1 \mathrm{H}), 5.44-5.38(\mathrm{~d}$, $18.5 \mathrm{~Hz}, 1 \mathrm{H}), 3.18-3.13(\mathrm{~d}, 2.6 \mathrm{~Hz}, 1 \mathrm{H}), 1.75-1.65(\mathrm{~m}, 4 \mathrm{H}), 1.63-1.56(\mathrm{~m}, 2 \mathrm{H}), 1.54-1.47(\mathrm{~m}$, $3 \mathrm{H}), 1.46-1.35(\mathrm{~m}, 3 \mathrm{H}), 1.29-1.25(\mathrm{~d}, 6.2 \mathrm{~Hz}, 12 \mathrm{H}), 1.22-1.18(\mathrm{~m}, 2 \mathrm{H}), 1.17-1.08(\mathrm{~m}, 3 \mathrm{H}), 1.01$

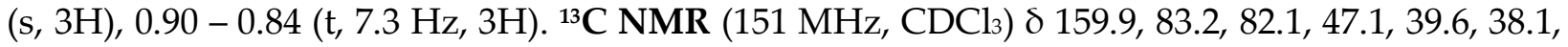

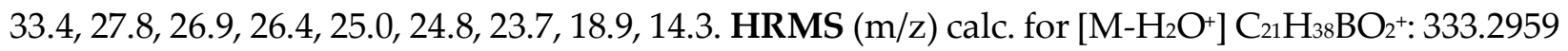
found: 333.2960. IR $\left(\mathrm{cm}^{-1}\right) 3054(\mathrm{w}), 1421(\mathrm{w}), 1265(\mathrm{~s}), 896(\mathrm{w}), 740(\mathrm{~s}) 705(\mathrm{~m}) .[\alpha]_{\mathrm{D}^{23}}-2.3(\mathrm{c}=$ $\left.0.89, \mathrm{CH}_{2} \mathrm{Cl}_{2}, 1=1 \mathrm{dm}\right)$.

Phenomenex lux Amylose 1-AD Column: 97:3 $\mathrm{CO}_{2}: \mathrm{iPrOH} ; 1.0 \mathrm{~mL} / \mathrm{min} ; 210 \mathrm{~nm}$.

Racemic material:
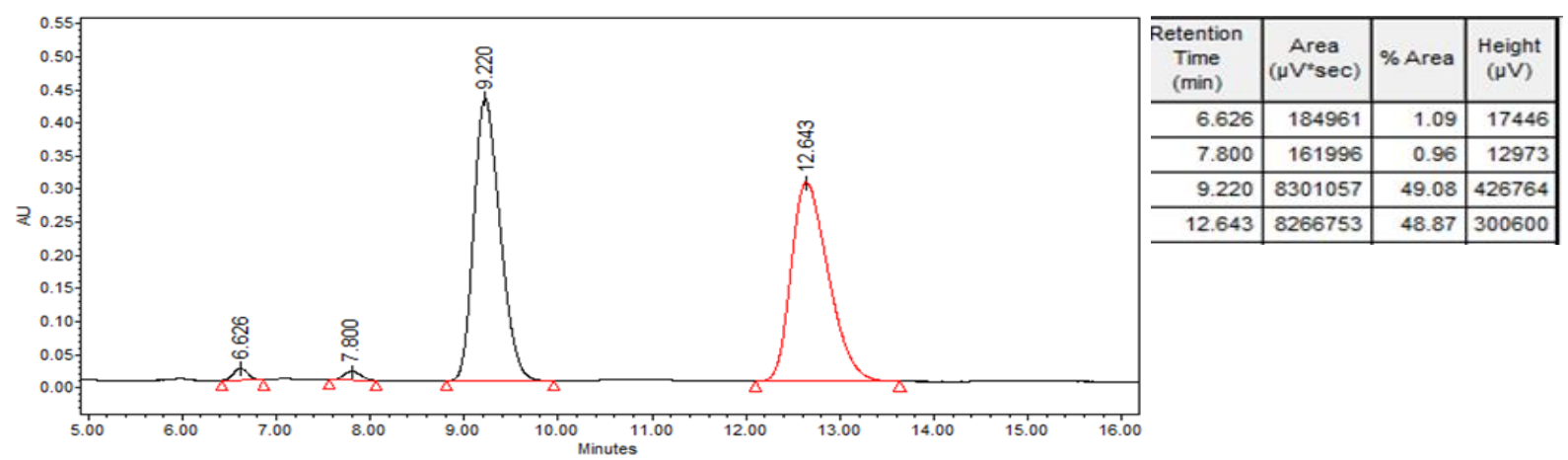

Enantioenriched material: 99:1 er

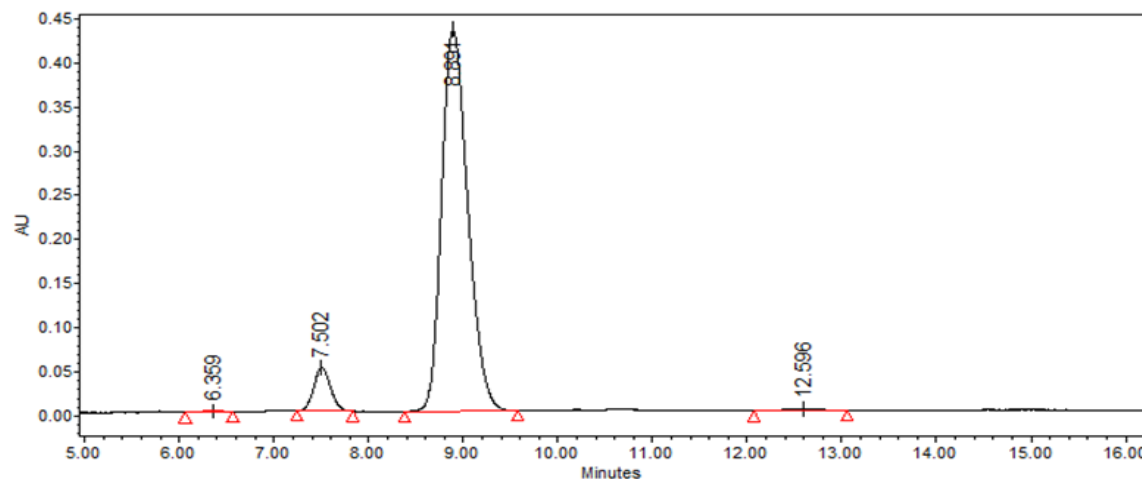

\begin{tabular}{r|r|r|r|}
\hline $\begin{array}{c}\text { Retention } \\
\text { Time } \\
(\mathrm{min})\end{array}$ & $\begin{array}{c}\text { Area } \\
\left(\mu \mathrm{V}^{*} \mathrm{sec}\right)\end{array}$ & \% Area & $\begin{array}{c}\text { Height } \\
(\mu \mathrm{V})\end{array}$ \\
\hline 6.359 & 20156 & 0.22 & 1520 \\
\hline 7.502 & 610893 & 6.79 & 49455 \\
\hline 8.891 & 8293922 & 92.18 & 431629 \\
\hline 12.596 & 72438 & 0.81 & 2902 \\
\hline
\end{tabular}


tert-butyl-4-((1R,2S)-1-hydroxy-2-methyl-2-((E)-2-(4,4,5,5-tetramethyl-1,3,2-dioxaborolan-2yl)vinyl)hexyl)piperidine-1-carboxylate (5h)

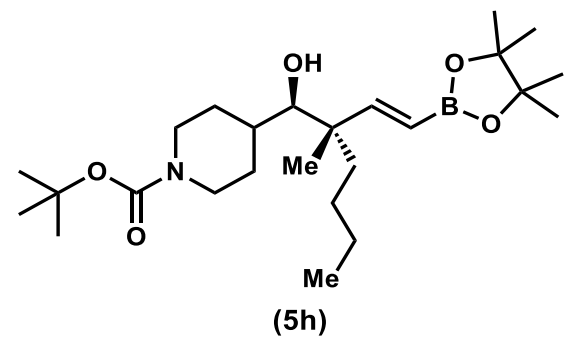

Prepared according to General Method III. Purified by silica gel column chromatography (Hexanes/Ethyl Acetate 25:1, 20:1, 15:1, 10:1, 8:1) to give the product (22.0 mg, 96\% yield, >99:1 er, 10:1 dr) as a colorless oil. ${ }^{1} \mathbf{H}$ NMR $\left(600 \mathrm{MHz}, \mathrm{CDCl}_{3}\right) \delta 6.59-6.51(\mathrm{~d}, 18.5 \mathrm{~Hz}, 1 \mathrm{H}), 5.47-5.40(\mathrm{~d}$, $18.5 \mathrm{~Hz}, 1 \mathrm{H}), 4.07$ (br s, 2H), $3.25-3.17(\mathrm{~m}, 1 \mathrm{H}), 2.63$ (br s, 2H), $1.64(\mathrm{~s}, 3 \mathrm{H}), 1.43(\mathrm{~s}, 12 \mathrm{H}), 1.42$ $1.37(\mathrm{~m}, 3 \mathrm{H}), 1.28-1.24(\mathrm{~m}, 16 \mathrm{H}), 1.18-1.11(\mathrm{~m}, 2 \mathrm{H}), 1.03(\mathrm{~s}, 3 \mathrm{H}), 0.89-0.84(\mathrm{t}, 7.3 \mathrm{~Hz}, 3 \mathrm{H}) .{ }^{13} \mathrm{C}$ NMR (151 MHz, $\left.\mathrm{CDCl}_{3}\right) \delta$ 159.3, 154.9, 100.1, 83.3, 80.9, 79.4, 47.0, 38.2, 31.8, 28.6, 26.3, 25.0, 24.8, 23.7, 14.2. HRMS (m/z) calc. for $\left[\mathrm{M}+\mathrm{H}^{+}\right] \mathrm{C}_{25} \mathrm{H}_{47} \mathrm{BNO}_{5}{ }^{+}: 452.3542$ found: 452.3541 . IR $\left(\mathrm{cm}^{-1}\right) 3446$ (m), $2976(\mathrm{~s}), 2931$ (m), $1673(\mathrm{~s}), 1469(\mathrm{w}), 1351(\mathrm{~s}), 1147(\mathrm{~s}), 970(\mathrm{w}), 851(\mathrm{w}) .[\alpha]_{\mathrm{D}^{23}}-10.7(\mathrm{c}=1.10$, $\left.\mathrm{CH}_{2} \mathrm{Cl}_{2}, \mathrm{l}=1 \mathrm{dm}\right)$.

Phenomenex lux Amylose 1-AD Column: 90:10 CO$:: \mathrm{PrOH} ; 1.0 \mathrm{~mL} / \mathrm{min} ; 210 \mathrm{~nm}$.

\section{Racemic material:}
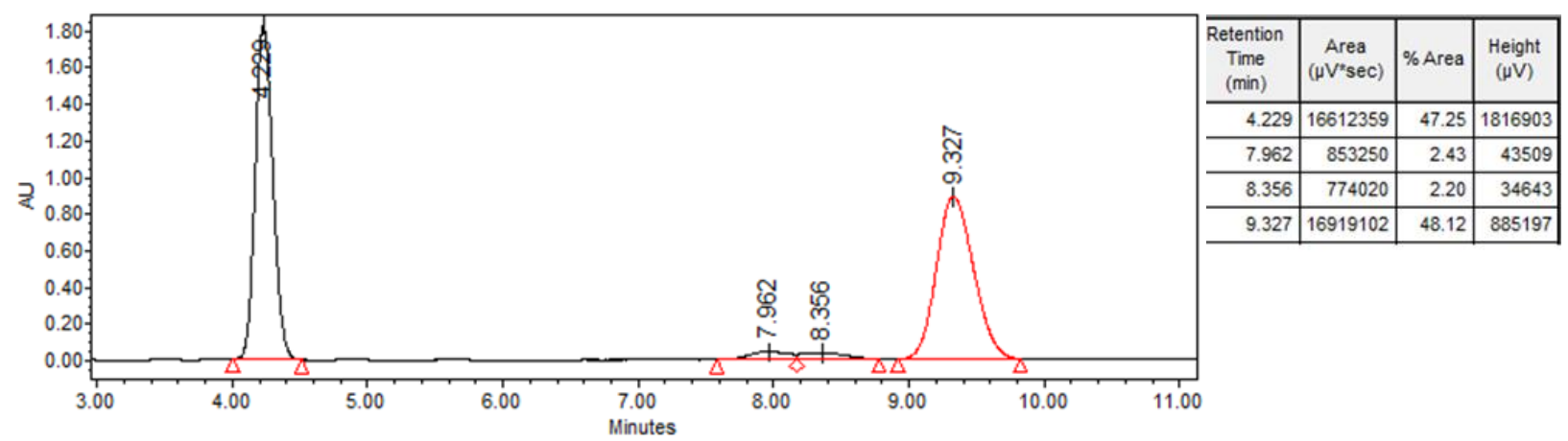

Enantioenriched material: >99:1 er

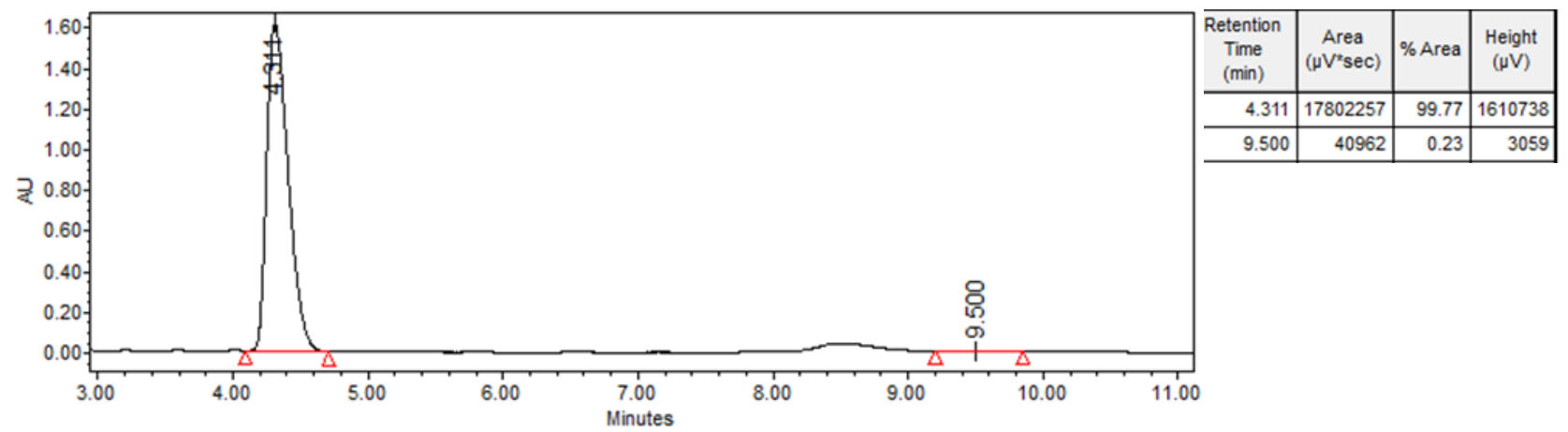


(1R,2S)-1-cyclopropyl-2-methyl-2-((E)-2-(4,4,5,5-tetramethyl-1,3,2-dioxaborolan-2-yl)vinyl)hexan-1-ol (5i)

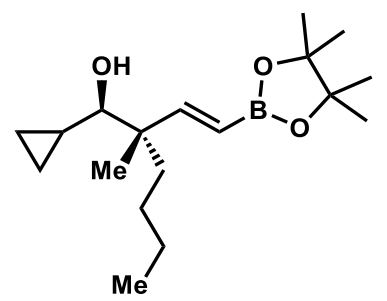

(5i)

Prepared according to General Method III. Purified by silica gel column chromatography (Hexanes/Ethyl Acetate 25:1, 20:1, 15:1, 10:1) to give the product (14.8 mg, 95\% yield, >99:1 er, 5:1 dr) as a colorless oil.

\section{Major Diastereomer}

${ }^{1} \mathrm{H}$ NMR $\left(600 \mathrm{MHz}, \mathrm{CDCl}_{3}\right) \delta 6.66-6.61(\mathrm{~d}, 18.6 \mathrm{~Hz}, 1 \mathrm{H}), 5.48-5.43(\mathrm{~d}, 18.5 \mathrm{~Hz}, 1 \mathrm{H}), 2.70$ - 2.65 $(\mathrm{d}, 8.6 \mathrm{~Hz}, 1 \mathrm{H}), 1.60-1.52(\mathrm{~m}, 2 \mathrm{H}), 1.49-1.41(\mathrm{~m}, 2 \mathrm{H}), 1.27(\mathrm{~s}, 12 \mathrm{H}), 1.19-1.13(\mathrm{~m}, 2 \mathrm{H}), 1.06$ (s, $3 \mathrm{H}), 0.95-0.90(\mathrm{~m}, 1 \mathrm{H}), 0.90-0.85(\mathrm{t}, 7.3 \mathrm{~Hz}, 3 \mathrm{H}), 0.56-0.49(\mathrm{~m}, 1 \mathrm{H}), 0.47-0.41(\mathrm{~m}, 1 \mathrm{H}), 0.31-$ $0.26(\mathrm{~m}, 1 \mathrm{H}), 0.26-0.20(\mathrm{~m}, 1 \mathrm{H}) .{ }^{13} \mathrm{C}$ NMR $\left(151 \mathrm{MHz}, \mathrm{CDCl}_{3}\right) \delta$ 159.2, 83.2, 82.5, 47.1, 36.5, 26.4, 25.0, 24.9, 23.7, 18.6, 13.8, 5.2, 2.0.

\section{Minor Diastereomer}

${ }^{1}$ H NMR $\left(600 \mathrm{MHz}, \mathrm{CDCl}_{3}\right) \delta 6.70$ - $6.63(\mathrm{~d}, 18.2 \mathrm{~Hz}, 1 \mathrm{H}), 5.51-5.44(\mathrm{~d}, 18.5 \mathrm{~Hz}, 1 \mathrm{H}), 2.64-2.61$ $(\mathrm{d}, 9.0 \mathrm{~Hz}, 1 \mathrm{H}), 1.60-1.52(\mathrm{~m}, 2 \mathrm{H}), 1.50-1.41(\mathrm{~m}, 2 \mathrm{H}), 1.27(\mathrm{~s}, 12 \mathrm{H}), 1.19-1.13(\mathrm{~m}, 2 \mathrm{H}), 1.05$ (s, $3 \mathrm{H}), 0.95-0.90(\mathrm{~m}, 1 \mathrm{H}), 0.90-0.85(\mathrm{t}, 7.3 \mathrm{~Hz}, 3 \mathrm{H}), 0.61-0.56(\mathrm{~m}, 1 \mathrm{H}), 0.47-0.41(\mathrm{~m}, 1 \mathrm{H}), 0.31-$ $0.26(\mathrm{~m}, 1 \mathrm{H}), 0.26-0.20(\mathrm{~m}, 1 \mathrm{H}) .{ }^{13} \mathrm{C}$ NMR $\left(151 \mathrm{MHz}, \mathrm{CDCl}_{3}\right) \delta$ 159.7, 83.3, 82.3, 47.3, 37.2, 26.4, 25.0, 24.9, 23.7, 17.8, 13.5, 5.4, 1.8.

HRMS (m/z) calc. for [M-H $\left.\mathrm{O}^{+}\right] \mathrm{C}_{18} \mathrm{H}_{32} \mathrm{BO}_{2}{ }^{+}: 291.2490$ found: 291.2499. IR (cm $\left.{ }^{-1}\right) 3432$ (s), 2977 (m), $2932(\mathrm{~m}), 1633(\mathrm{~m}), 1351(\mathrm{~m}), 1147(\mathrm{~m}), 970(\mathrm{w}), 850(\mathrm{w}) .[\alpha]_{\mathrm{D}^{23}}=-12.6\left(\mathrm{c}=0.74, \mathrm{CH}_{2} \mathrm{Cl}_{2}, 1=1 \mathrm{dm}\right)$.

Diacel CHIRALPAK IC Column: 97:3 $\mathrm{CO}_{2}: \mathrm{MeOH} ; 1.0 \mathrm{~mL} / \mathrm{min} ; 210 \mathrm{~nm}$.

Racemic material:

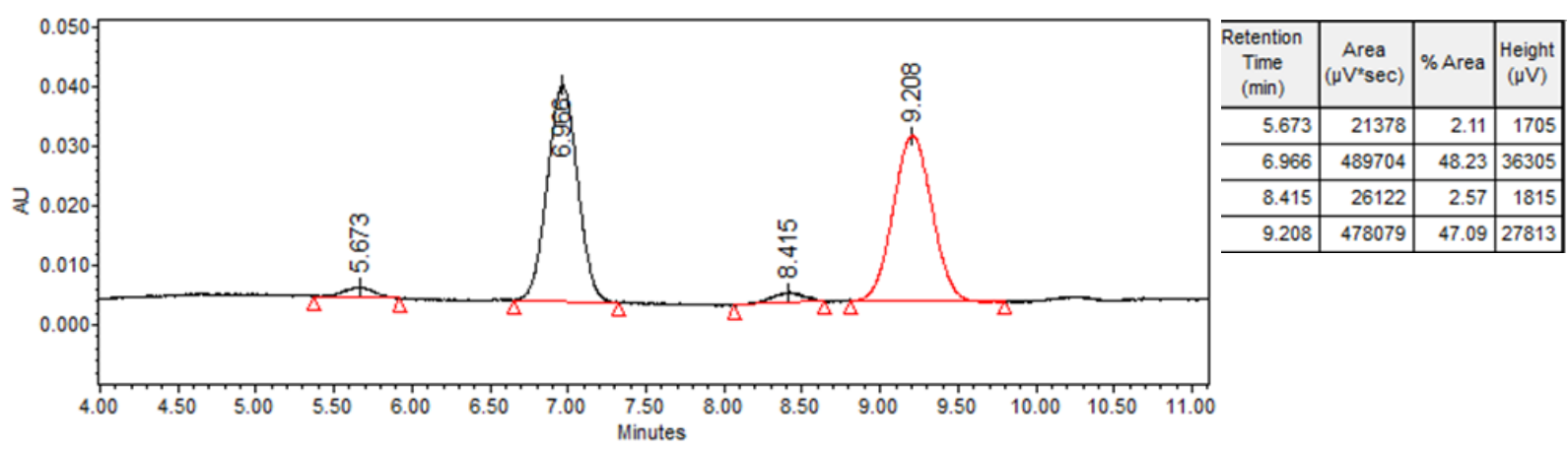

Enantioenriched material: >99:1 er 


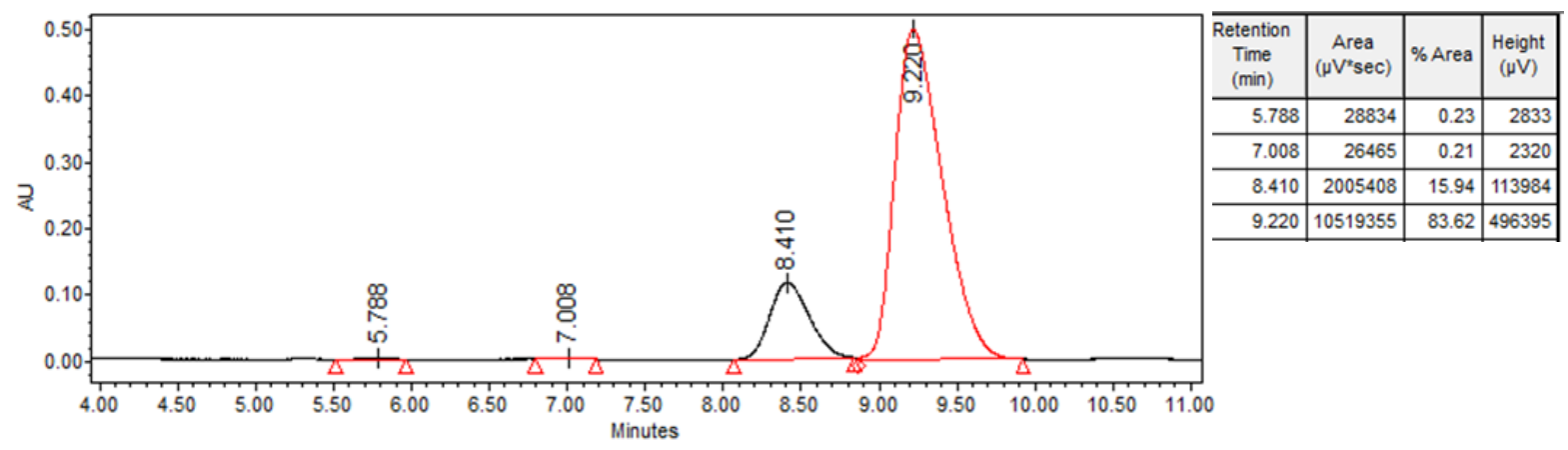

(4R,5S)-2,5-dimethyl-5-((E)-2-(4,4,5,5-tetramethyl-1,3,2-dioxaborolan-2-yl)vinyl)nonan-4-ol (5j)

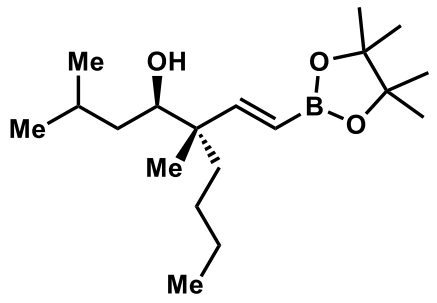

(5j)

Prepared according to General Method III. Purified by silica gel column chromatography (Hexanes/Ethyl Acetate 25:1, 20:1, 15:1, 10:1) to give the product (16.2 mg, 99\% yield, >99:1 er, 4:1 dr) as a colorless oil.

\section{Major Diastereomer}

${ }^{1}$ H NMR (600 MHz, CDCl3) d 6.51 - 6.46 (d, 18.6 Hz, 1H), 5.45 - 5.40 (d, 18.6 Hz, 1H), 3.44 - 3.39 $(\mathrm{d}, 8.4 \mathrm{~Hz}, 1 \mathrm{H}), 1.82-1.73(\mathrm{~m}, 1 \mathrm{H}), 1.47-1.32(\mathrm{~m}, 4 \mathrm{H}), 1.27(\mathrm{~s}, 12 \mathrm{H}), 1.21-1.09(\mathrm{~m}, 4 \mathrm{H}), 0.97$ (s, 3H), $0.94-0.91(\mathrm{~m}, 3 \mathrm{H}), 0.90-0.84(\mathrm{~m}, 6 \mathrm{H}) .{ }^{13} \mathrm{C}$ NMR (151 MHz, CDCl3) ठ 159.2, 83.3, 75.9, 46.2, $41.0,36.5,26.3,25.1,25.0,24.9,24.3,23.7,21.5,18.3$.

\section{Minor Diastereomer}

${ }^{1} \mathbf{H}$ NMR $\left(600 \mathrm{MHz}, \mathrm{CDCl}_{3}\right) \delta 6.55$ - 6.51 (d, $\left.18.7 \mathrm{~Hz}, 1 \mathrm{H}\right), 5.49$ - 5.42 (d, $\left.18.7 \mathrm{~Hz}, 1 \mathrm{H}\right), 3.44-3.39$ $(\mathrm{d}, 8.4 \mathrm{~Hz}, 1 \mathrm{H}), 1.82-1.73(\mathrm{~m}, 1 \mathrm{H}), 1.47-1.32(\mathrm{~m}, 4 \mathrm{H}), 1.27(\mathrm{~s}, 12 \mathrm{H}), 1.21-1.09(\mathrm{~m}, 4 \mathrm{H}), 0.97$ (s,

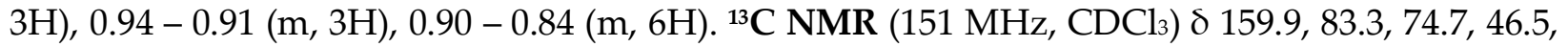
$40.0,37.0,26.4,25.1,25.0,25.0,24.3,23.7,21.4,16.9$.

HRMS (m/z) calc. for [M-H $\left.\mathrm{H}^{+}\right] \mathrm{C}_{19} \mathrm{H}_{36} \mathrm{BO}_{3}: 323.2763$ found: 323.2770. IR (cm-1) 3432 (s), $2956(\mathrm{~m})$, $2932(\mathrm{~m}), 1633(\mathrm{~m}), 1351(\mathrm{~m}), 1147(\mathrm{~m}), 970(\mathrm{w}), 850(\mathrm{w}) .[\alpha]_{\mathrm{D}^{23}}=-13.2\left(\mathrm{c}=0.81, \mathrm{CH}_{2} \mathrm{Cl}_{2}, 1=1 \mathrm{dm}\right)$.

Phenomenex lux Amylose 1-AD Column: 96:4 $\mathrm{CO}_{2}: \mathrm{iPrOH} ; 1.0 \mathrm{~mL} / \mathrm{min} ; 210 \mathrm{~nm}$. Racemic material: 

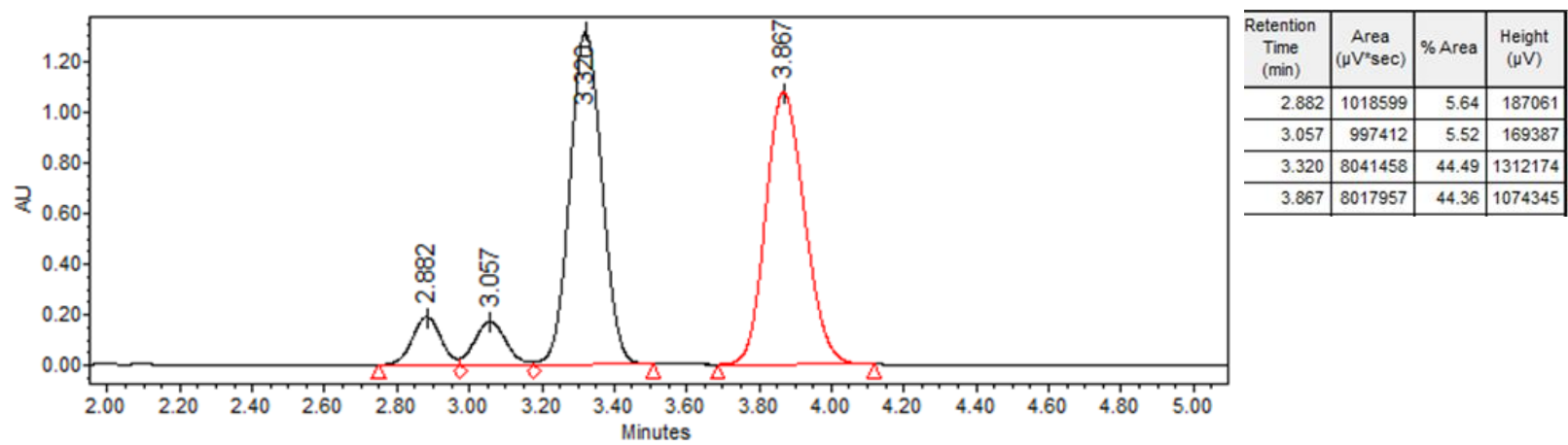

\section{Enantioenriched material:}

Major: >99:1 er

Minor: $97: 3 \mathrm{er}$

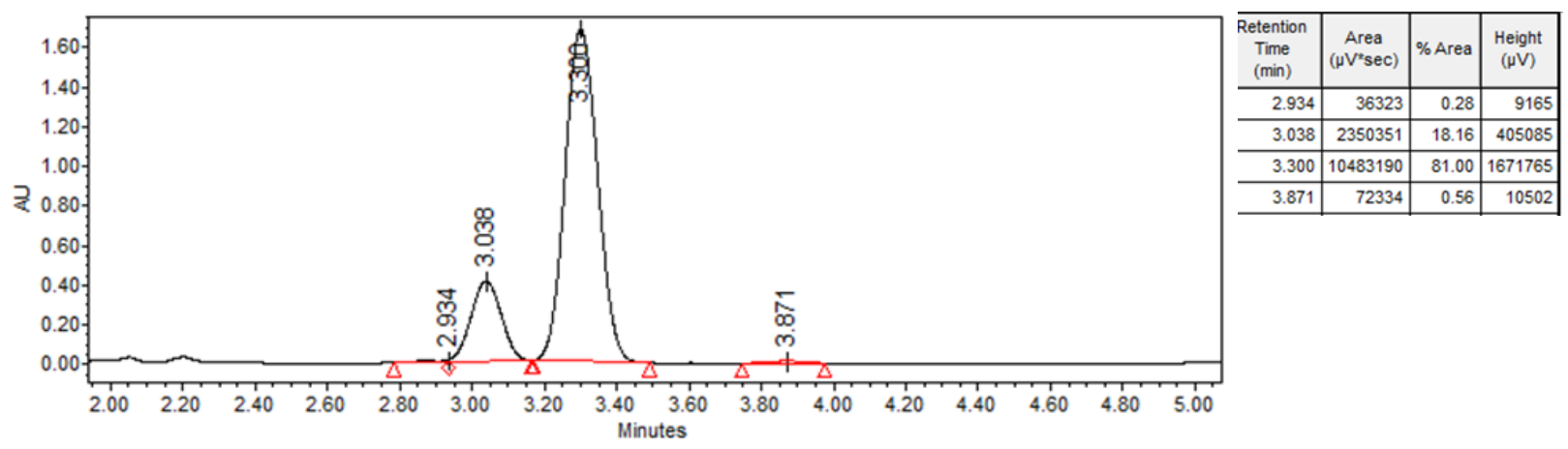

(5R,6S)-6-methyl-6-((E)-2-(4,4,5,5-tetramethyl-1,3,2-dioxaborolan-2-yl)vinyl)dec-1-en-5-ol (5k)

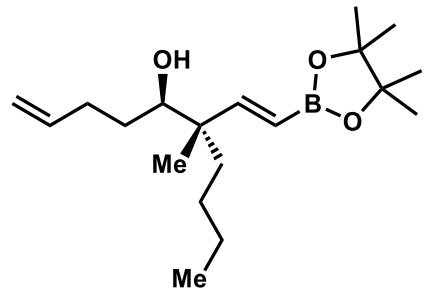

$(5 k)$

Prepared according to General Method III. Purified by silica gel column chromatography (Hexanes/Ethyl Acetate 25:1, 20:1, 15:1, 10:1) to give the product (15.8 mg, 99\% yield, 98.5:1.5 er, 6:1 dr) as a colorless oil. ${ }^{1} \mathrm{H}$ NMR $\left(600 \mathrm{MHz}, \mathrm{CDCl}_{3}\right) \delta 6.52-6.45(\mathrm{~d}, 18.5 \mathrm{~Hz}, 1 \mathrm{H}), 5.88-5.76(\mathrm{~m}$, $1 \mathrm{H}), 5.47-5.39(\mathrm{~d}, 18.5 \mathrm{~Hz}, 1 \mathrm{H}), 5.17-5.00(\mathrm{dd}, 17.2,1.8 \mathrm{~Hz}, 1 \mathrm{H}), 4.99-4.93(\mathrm{dd}, 10.2,1.0 \mathrm{~Hz}, 1 \mathrm{H})$, $3.40-3.31(\mathrm{~d}, 9.4 \mathrm{~Hz}, 1 \mathrm{H}), 2.38-2.24(\mathrm{~m}, 1 \mathrm{H}), 2.16-2.03(\mathrm{~m}, 1 \mathrm{H}), 1.67-1.51(\mathrm{~m}, 2 \mathrm{H}), 1.47-1.37$ $(\mathrm{m}, 4 \mathrm{H}), 1.27(\mathrm{~s}, 12 \mathrm{H}), 1.20-1.11(\mathrm{~m}, 2 \mathrm{H}), 0.98(\mathrm{~s}, 3 \mathrm{H}), 0.91-0.81(\mathrm{t}, 7.2 \mathrm{~Hz}, 3 \mathrm{H}) .{ }^{13} \mathrm{C}$ NMR $(151$ $\left.\mathrm{MHz}_{\mathrm{CDCl}}\right) \delta$ d 159.0, 138.9, 114.9, 83.3, 77.5, 46.3, 36.8, 31.4, 31.1, 26.3, 25.0, 24.9, 23.7, 17.9, 14.2. HRMS (m/z) calc. for [M- $\left.\mathrm{H}_{2} \mathrm{O}^{+}\right] \mathrm{C}_{19} \mathrm{H}_{34} \mathrm{BO}_{2}{ }^{+}: 305.2646$ found: 305.2650. IR (cm-1) $2957(\mathrm{~m}), 2931(\mathrm{~m})$, $2861(\mathrm{w}), 1633(\mathrm{w}), 1379(\mathrm{~m}), 1351(\mathrm{~s}), 1146(\mathrm{~m}), 911(\mathrm{w}), 851(\mathrm{w}) .[\alpha]_{\mathrm{D}^{23}}^{23}-2.5$ (c = 0.79, $\mathrm{CH}_{2} \mathrm{Cl}_{2}, 1$ $=1 \mathrm{dm})$. 
Phenomenex lux Amylose 1-AD Column: 95:5 $\mathrm{CO}_{2}: \mathrm{iPrOH} ; 1.0 \mathrm{~mL} / \mathrm{min} ; 210 \mathrm{~nm}$.

Racemic material:

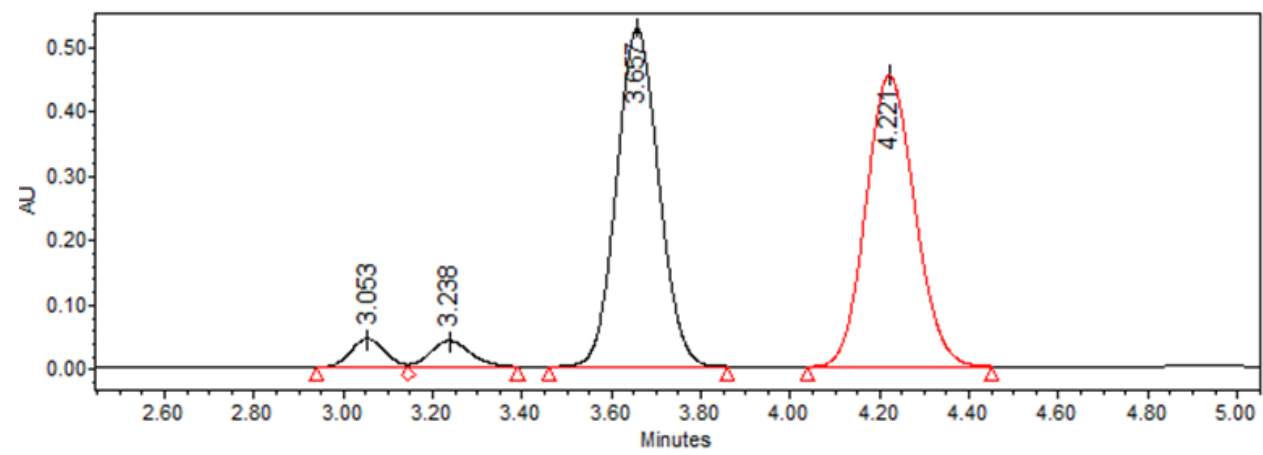

\begin{tabular}{|r|r|r|r|}
\hline $\begin{array}{c}\text { Retention } \\
\text { Time } \\
(\mathrm{min})\end{array}$ & $\begin{array}{c}\text { Area } \\
\left(\mu \mathrm{V}^{2} \mathrm{sec}\right)\end{array}$ & $\%$ Area & $\begin{array}{c}\text { Height } \\
(\mu \mathrm{V})\end{array}$ \\
\hline 3.053 & 231333 & 3.14 & 43829 \\
\hline 3.238 & 253397 & 3.44 & 40538 \\
\hline 3.657 & 3445657 & 46.78 & 524611 \\
\hline 4.221 & 3435380 & 46.64 & 452140 \\
\hline
\end{tabular}

Enantioenriched material: 98.5:1.5 er

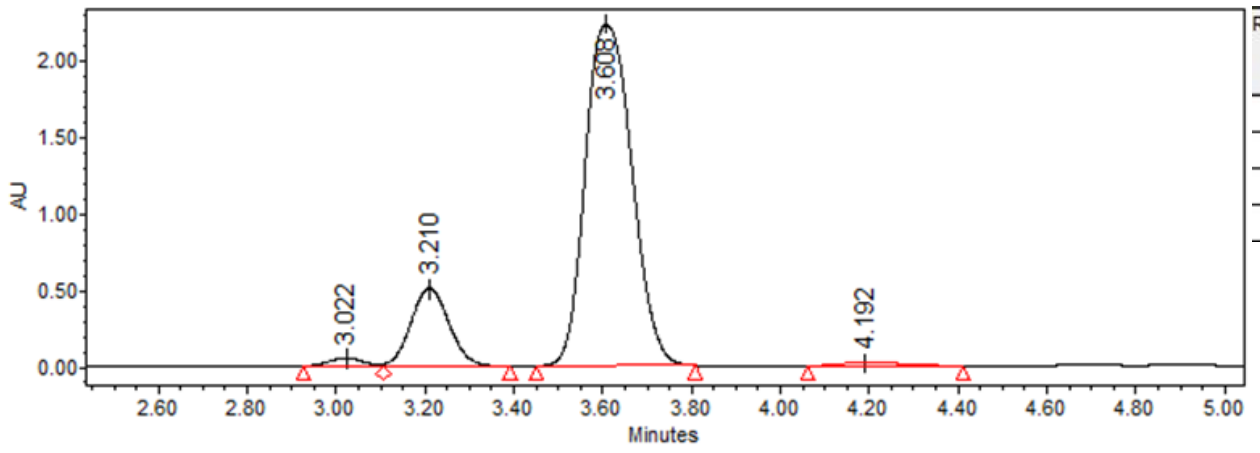

\begin{tabular}{|r|r|r|r|}
$\begin{array}{c}\text { Retention } \\
\text { Time } \\
(\mathrm{min})\end{array}$ & $\begin{array}{c}\text { Area } \\
\left(\mu \mathrm{V}^{*} \mathrm{sec}\right)\end{array}$ & $\%$ Area & $\begin{array}{c}\text { Height } \\
(\mu \mathrm{V})\end{array}$ \\
\hline 3.022 & 288153 & 1.45 & 52545 \\
\hline 3.210 & 3037200 & 15.27 & 511542 \\
\hline 3.608 & 16335593 & 82.11 & 2232551 \\
\hline 4.192 & 233461 & 1.17 & 21830 \\
\hline
\end{tabular}

(5S,6R,8S)-5,8,12-trimethyl-5-((E)-2-(4,4,5,5-tetramethyl-1,3,2-dioxaborolan-2-yl)vinyl)tridec-11-en-6-ol (5I)

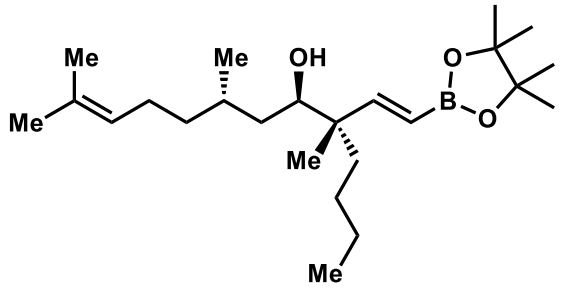

(5I)

Prepared according to General Method III. Purified by silica gel column chromatography (Hexanes/Ethyl Acetate 25:1, 20:1, 15:1, 10:1) to give the product (18.1 mg, 92\% yield, 3:1 dr) as a colorless oil.

\section{Major Diastereomer}

${ }^{1} \mathrm{H}$ NMR $\left(600 \mathrm{MHz}, \mathrm{CDCl}_{3}\right) \delta 6.54-6.46(\mathrm{~d}, 18.5 \mathrm{~Hz}, 1 \mathrm{H}), 5.47-5.42(\mathrm{~d}, 18.6 \mathrm{~Hz}, 1 \mathrm{H}), 5.15-5.19$ (m, 1H), $3.48-3.43(\mathrm{~m}, 1 \mathrm{H}), 2.07-1.99(\mathrm{~m}, 1 \mathrm{H}), 1.96-1.86(\mathrm{~m}, 1 \mathrm{H}), 1.70(\mathrm{~s}, 3 \mathrm{H}), 1.66(\mathrm{~s}, 1 \mathrm{H}), 1.62$ $(\mathrm{s}, 3 \mathrm{H}), 1.50-1.32(\mathrm{~m}, 5 \mathrm{H}), 1.30(\mathrm{~s}, 12 \mathrm{H}), 1.25-1.14(\mathrm{~m}, 5 \mathrm{H}), 1.09-1.03(\mathrm{~m}, 1 \mathrm{H}), 0.99(\mathrm{~s}, 3 \mathrm{H}), 0.96$ - $0.93(\mathrm{t}, 6.6 \mathrm{~Hz}, 3 \mathrm{H}), 0.92-0.83(\mathrm{~m}, 3 \mathrm{H}) .{ }^{13} \mathrm{C}$ NMR $\left(151 \mathrm{MHz}, \mathrm{CDCl}_{3}\right) \delta$ 159.3, 125.0, 83.3, 76.0, $46.4,39.5,36.5,35.7,29.9,26.3,25.9,25.0,24.9,23.7,21.0,18.1,17.8,17.0,14.2$. 


\section{Minor Diastereomer}

${ }^{1}$ H NMR (600 MHz, CDCl $)$ d 6.58 - 6.54 (d, 18.4 Hz, 1H), 5.50 - 5.45 (d, $\left.19.1 \mathrm{~Hz}, 1 \mathrm{H}\right)$, ), 5.15 - 5.19 $(\mathrm{m}, 1 \mathrm{H}), 3.48-3.43(\mathrm{~m}, 1 \mathrm{H}), 2.07-1.99(\mathrm{~m}, 1 \mathrm{H}), 1.96-1.86(\mathrm{~m}, 1 \mathrm{H}), 1.70(\mathrm{~s}, 3 \mathrm{H}), 1.66(\mathrm{~s}, 1 \mathrm{H}), 1.62$ $(\mathrm{s}, 3 \mathrm{H}), 1.50-1.32(\mathrm{~m}, 5 \mathrm{H}), 1.29(\mathrm{~s}, 12 \mathrm{H}), 1.25-1.14(\mathrm{~m}, 5 \mathrm{H}), 1.09-1.03(\mathrm{~m}, 1 \mathrm{H}), 0.99(\mathrm{~s}, 3 \mathrm{H}), 0.96$ - $0.93(\mathrm{t}, 6.6 \mathrm{~Hz}, 3 \mathrm{H}), 0.92-0.83(\mathrm{~m}, 3 \mathrm{H}) .{ }^{13} \mathrm{C}$ NMR $\left(151 \mathrm{MHz}, \mathrm{CDCl}_{3}\right) \delta$ 159.9, 131.2, 125.0, 83.3, 74.4, 46.5, 38.5, 38.3, 37.0, 29.3, 26.4, 25.7, 25.4, 24.9, 23.7, 18.8, 17.8, 15.4.

HRMS (m/z) calc. for [M+Na+] $\mathrm{C}_{24} \mathrm{H}_{45} \mathrm{BO}_{3} \mathrm{Na}^{+}: 415.3360$ found: $415.3348 . \mathbf{I R}\left(\mathrm{cm}^{-1}\right) 3432$ (s), 2959 (m), $2930(\mathrm{~m}), 1632(\mathrm{~s}), 1350(\mathrm{~s}), 1147(\mathrm{~s}), 850(\mathrm{w}) .[\alpha]_{\mathrm{D}^{23}}=-3.5\left(\mathrm{c}=0.84, \mathrm{CH}_{2} \mathrm{Cl}_{2}, 1=1 \mathrm{dm}\right)$.

(1S,2S,E)-1-(4-bromophenyl)-2-ethyl-2-methyl-4-(4,4,5,5-tetramethyl-1,3,2-dioxaborolan-2-yl)but-3-en-1ol (6b)

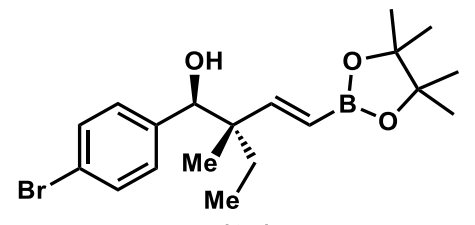

(6b)

Prepared according to General Method III using diboron 2b. Purified by silica gel column chromatography (Hexanes/Ethyl Acetate 25:1, 20:1, 15:1, 10:1) to give the product $(19.0 \mathrm{mg}, 96 \%$ yield, 98:2 er, 7:1 dr) as a colorless oil. ${ }^{1} \mathbf{H}$ NMR $\left(600 \mathrm{MHz}, \mathrm{CDCl}_{3}\right) \delta 7.43-7.40(\mathrm{~d}, 8.3 \mathrm{~Hz}, 2 \mathrm{H})$, $7.18-7.14(\mathrm{~d}, 8.3 \mathrm{~Hz}, 2 \mathrm{H}), 6.63-6.56(\mathrm{~d}, 18.5 \mathrm{~Hz}, 1 \mathrm{H}), 5.55-5.48(\mathrm{~d}, 18.5 \mathrm{~Hz}, 1 \mathrm{H}), 4.44(\mathrm{~s}, 1 \mathrm{H})$, $2.01(\mathrm{~s}, 1 \mathrm{H}), 1.50-1.43(\mathrm{~m}, 1 \mathrm{H}), 1.28(\mathrm{~s}, 12 \mathrm{H}), 1.24-1.18(\mathrm{~m}, 1 \mathrm{H}), 0.86(\mathrm{~s}, 3 \mathrm{H}), 0.76-0.70(\mathrm{t}, 7.5 \mathrm{~Hz}$, 3H). ${ }^{13} \mathrm{C}$ NMR $\left(151 \mathrm{MHz}, \mathrm{CDCl}_{3}\right) \delta 158.6,139.5,130.7,129.9,121.5,83.4,79.3,47.8,29.9,24.9,15.3$, 8.6. HRMS (m/z) calc. for $\left[\mathrm{M}-\mathrm{H}_{2} \mathrm{O}^{+}\right] \mathrm{C}_{19} \mathrm{H}_{27} \mathrm{BBrO}_{2}{ }^{+}: 377.1282$ found: 377.1283 . IR $\left(\mathrm{cm}^{-1}\right) 3466(\mathrm{~m})$, $2976(\mathrm{~m}), 1633(\mathrm{~m}), 1483(\mathrm{w}), 1380(\mathrm{~m}), 1266(\mathrm{~m}), 1145(\mathrm{~s}), 1010(\mathrm{~m}), 851(\mathrm{~m}), 739(\mathrm{~m}) .[\alpha]_{\mathrm{D}^{23}}=-$ $33.4\left(\mathrm{c}=0.95, \mathrm{CH}_{2} \mathrm{Cl}_{2}, 1=1 \mathrm{dm}\right)$.

Diacel CHIRALPAK IE Column: 98:2 hexanes:iPrOH; $0.35 \mathrm{~mL} / \mathrm{min} ; 210 \mathrm{~nm}$.

\section{Racemic material:}

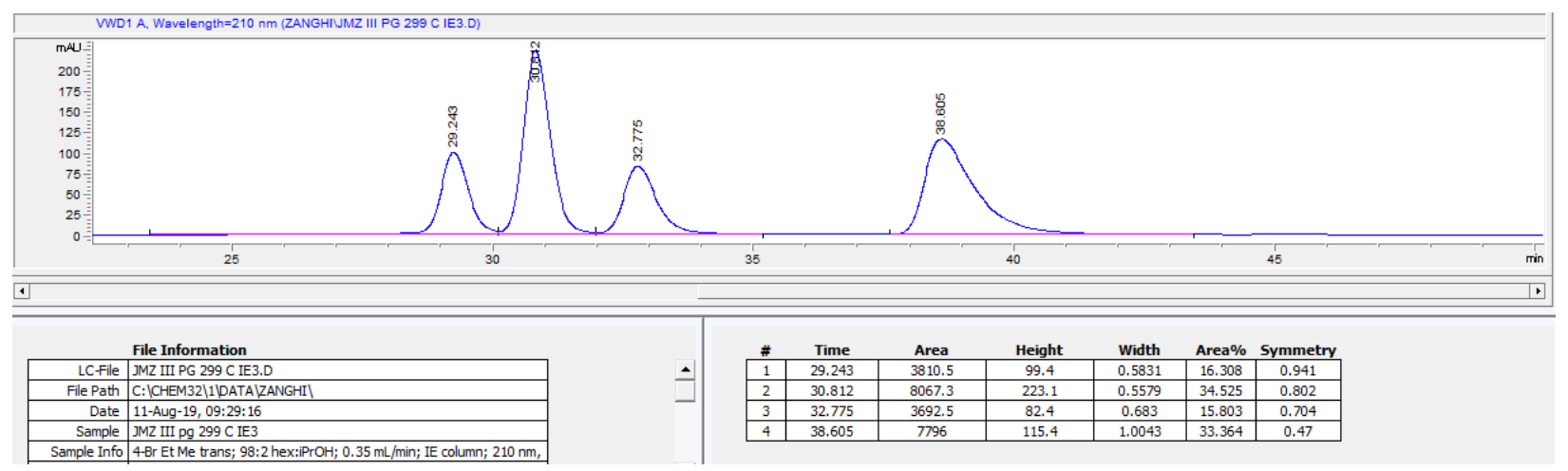

Enantioenriched material: 98:2 er 


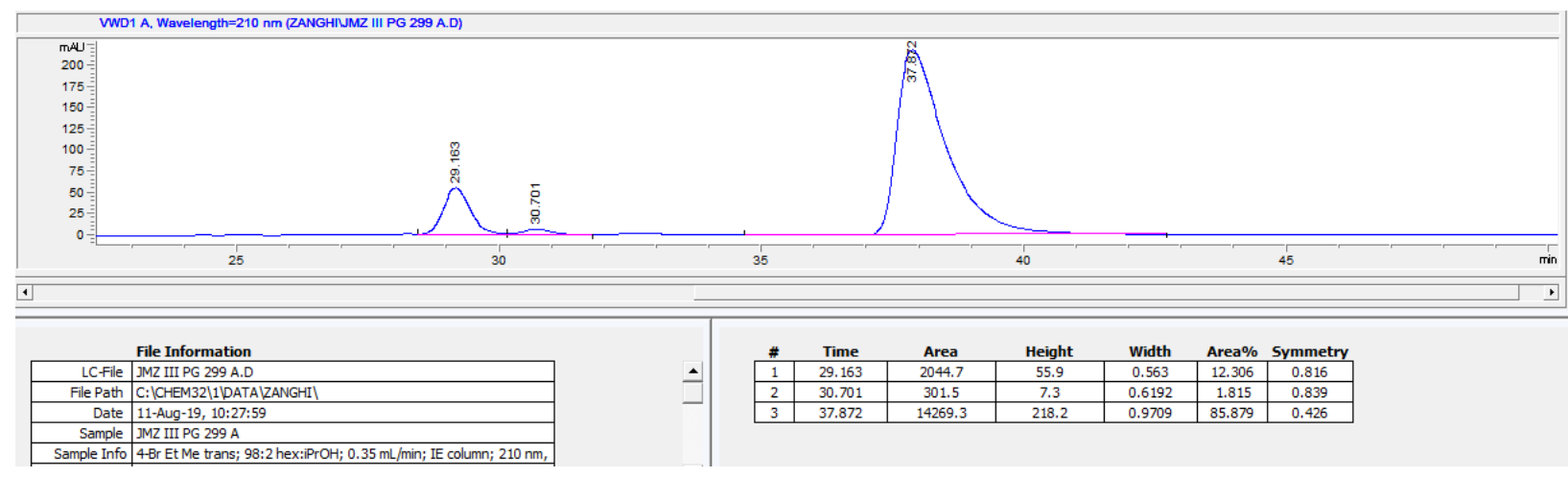

(1S,2R,E)-1-(4-bromophenyl)-2-ethyl-2-methyl-4-(4,4,5,5-tetramethyl-1,3,2-dioxaborolan-2-yl)but-3-en-1ol $(6 c)$

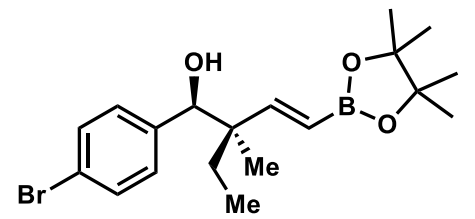

(6c)

Prepared according to General Method III using diboron 2c. Purified by silica gel column chromatography (Hexanes/Ethyl Acetate 25:1, 20:1, 15:1, 10:1) to give the product (19.8 $\mathrm{mg}, 99 \%$ yield, 98.5:1.5 er, 6:1 dr) as a colorless oil. ${ }^{1} \mathbf{H}$ NMR $\left(600 \mathrm{MHz}, \mathrm{CDCl}_{3}\right) \delta 7.44-7.39(\mathrm{~d}, 8.4 \mathrm{~Hz}, 2 \mathrm{H})$, $7.18-7.12(\mathrm{~d}, 8.4 \mathrm{~Hz}, 2 \mathrm{H}), 6.59-651(\mathrm{~d}, 18.5 \mathrm{~Hz}, 1 \mathrm{H}), 5.45-5.37(\mathrm{~d}, 18.5 \mathrm{~Hz}, 1 \mathrm{H}), 4.48(\mathrm{~s}, 1 \mathrm{H}), 2.09$ (s, 1H), $1.59-1.51(\mathrm{~m}, 1 \mathrm{H}), 1.43-1.37(\mathrm{~m}, 1 \mathrm{H}), 1.29(\mathrm{~s}, 12 \mathrm{H}), 0.98(\mathrm{~s}, 3 \mathrm{H}), 0.82-0.75(\mathrm{t}, 7.5 \mathrm{~Hz}, 3 \mathrm{H})$. ${ }^{13} \mathrm{C}$ NMR (151 MHz, $\left.\mathrm{CDCl}_{3}\right) \delta$ 157.6, 140.2, 130.6, 129.6, 121.3, 83.3, 80.0, 47.0, 28.1, 25.0, 24.8, 18.0, 8.6. HRMS (m/z) calc. for $\left[\mathrm{M}-\mathrm{H}_{2} \mathrm{O}^{+}\right] \mathrm{C}_{19} \mathrm{H}_{27} \mathrm{BBrO}_{2}{ }^{+}: 377.1282$ found: 377.1283 . IR $\left(\mathrm{cm}^{-1}\right) 3466(\mathrm{~m})$, $2976(\mathrm{~m}), 1633(\mathrm{~m}), 1372(\mathrm{~m}), 1352(\mathrm{~s}), 1260(\mathrm{~m}), 1145(\mathrm{~s}), 1010(\mathrm{~m}), 739(\mathrm{~m}) .[\alpha]_{\mathrm{D}^{23}}=-2.4(\mathrm{c}=0.99$, $\left.\mathrm{CH}_{2} \mathrm{Cl}_{2}, \mathrm{l}=1 \mathrm{dm}\right)$.

Diacel CHIRALPAK IE Column: $98: 2$ hexanes:iPrOH; $0.35 \mathrm{~mL} / \mathrm{min} ; 210 \mathrm{~nm}$.

\section{Racemic material:}

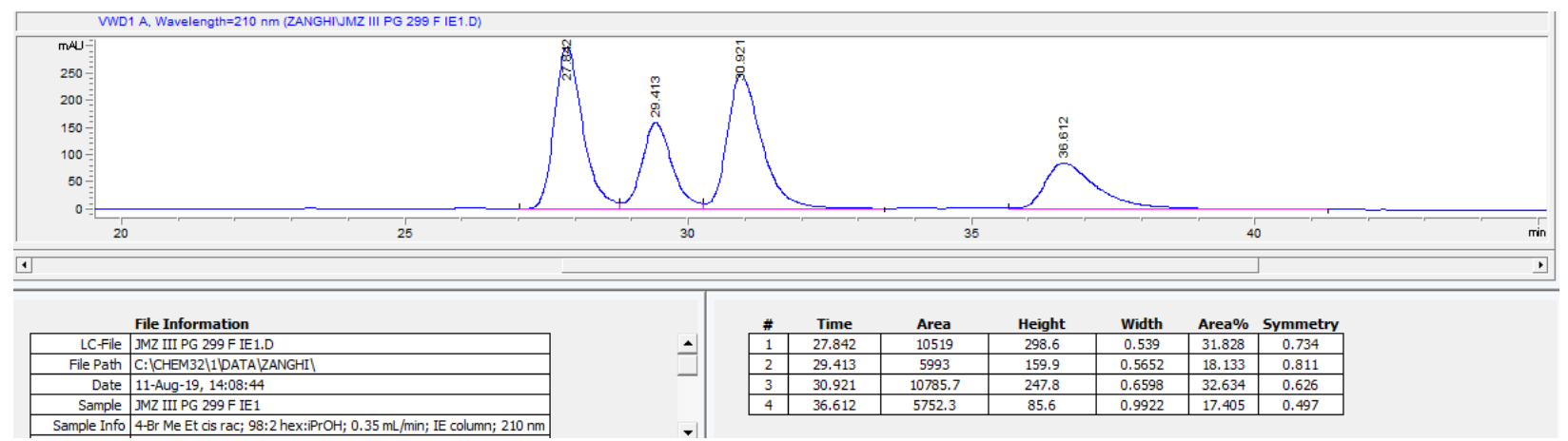

Enantioenriched material: >99:1 er 


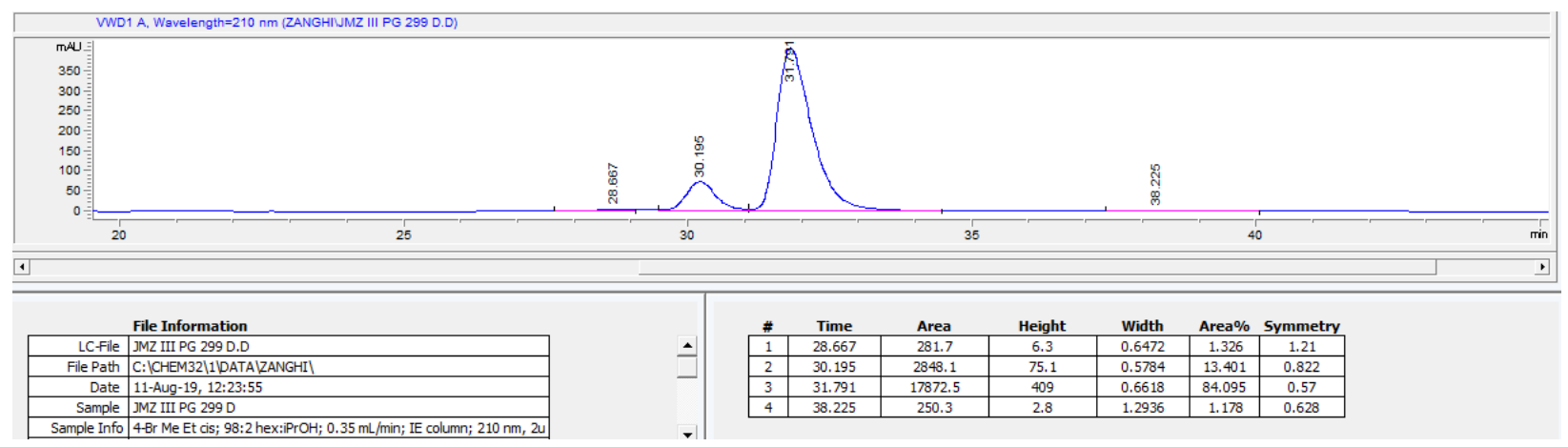

Methyl-4-((1R,2S,E)-2-cyclohexyl-1-hydroxy-2-methyl-4-(4,4,5,5-tetramethyl-1,3,2-dioxaborolan-2-yl)but3-en-1-yl)benzoate (6d)

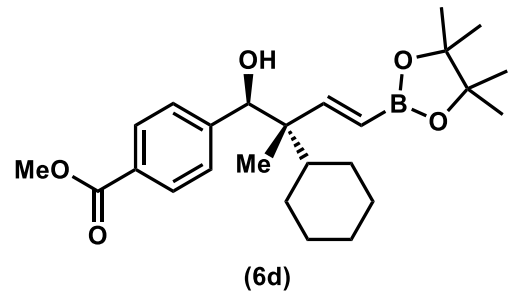

Prepared according to General Method III using diboron 2d. Purified by silica gel column chromatography (Hexanes/Ethyl Acetate 25:1, 20:1, 15:1, 10:1) to give the product (21.2 mg, 99\% yield, 98:2 er, 11:1 dr) as a colorless oil. ${ }^{1} \mathbf{H}$ NMR $\left(600 \mathrm{MHz}, \mathrm{CDCl}_{3}\right) \delta 7.94-7.87(\mathrm{~d}, 8.1 \mathrm{~Hz}, 2 \mathrm{H})$, $7.34-7.28(\mathrm{~d}, 8.1 \mathrm{~Hz}, 2 \mathrm{H}), 6.84-6.76(\mathrm{~d}, 18.6 \mathrm{~Hz}, 1 \mathrm{H}), 5.24-5.16(\mathrm{~d}, 18.6 \mathrm{~Hz}, 1 \mathrm{H}), 4.84(\mathrm{~s}, 1 \mathrm{H})$, $3.89(\mathrm{~s}, 3 \mathrm{H}), 2.00(\mathrm{~s}, 1 \mathrm{H}), 1.84-1.74(\mathrm{~m}, 3 \mathrm{H}), 1.74-1.68(\mathrm{~m}, 2 \mathrm{H}), 1.68-1.58(\mathrm{~m}, 2 \mathrm{H}), 1.27(\mathrm{~s}, 12 \mathrm{H})$, $1.23-1.18(\mathrm{~m}, 2 \mathrm{H}), 1.15-1.08(\mathrm{~m}, 1 \mathrm{H}), 1.05-0.95(\mathrm{~m}, 3 \mathrm{H}), 0.73(\mathrm{~s}, 3 \mathrm{H}) .{ }^{13} \mathrm{C} \mathrm{NMR}\left(151 \mathrm{MHz}, \mathrm{CDCl}_{3}\right)$ ठ 167.2, 157.1, 146.9, 129.2, 128.6, 128.3, 83.3, 52.2, 49.8, 41.8, 28.6, 27.2, 27.1, 27.1, 26.8, 25.0, 24.8, 14.8. HRMS (m/z) calc. for [M- $\left.\mathrm{H}_{2} \mathrm{O}^{+}\right] \mathrm{C}_{25} \mathrm{H}_{36} \mathrm{BO}_{4}{ }^{+}: 411.2701$ found: 411.2718 . IR (cm $\left.{ }^{-1}\right) 3446(\mathrm{~s}), 2976$ (m), $2931(\mathrm{~m}), 1633(\mathrm{~m}), 1351(\mathrm{~s}), 1146(\mathrm{~s}), 850(\mathrm{w}), 700(\mathrm{w}) .[\alpha]_{\mathrm{D}^{23}}=-48.4\left(\mathrm{c}=1.07, \mathrm{CH}_{2} \mathrm{Cl}_{2}, \mathrm{l}=1\right.$ $\mathrm{dm})$.

Diacel CHIRALPAK IA Column: 94:5 hex:iPrOH; $0.5 \mathrm{~mL} / \mathrm{min} ; 210 \mathrm{~nm}$.

\section{Racemic material:}

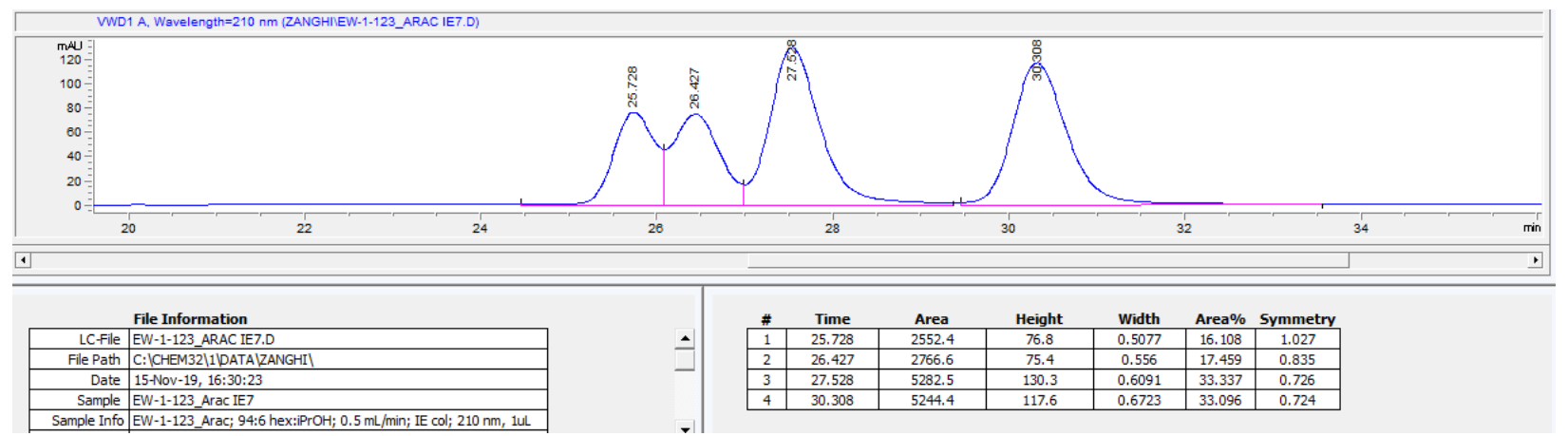


Enantioenriched material: 98:2 er

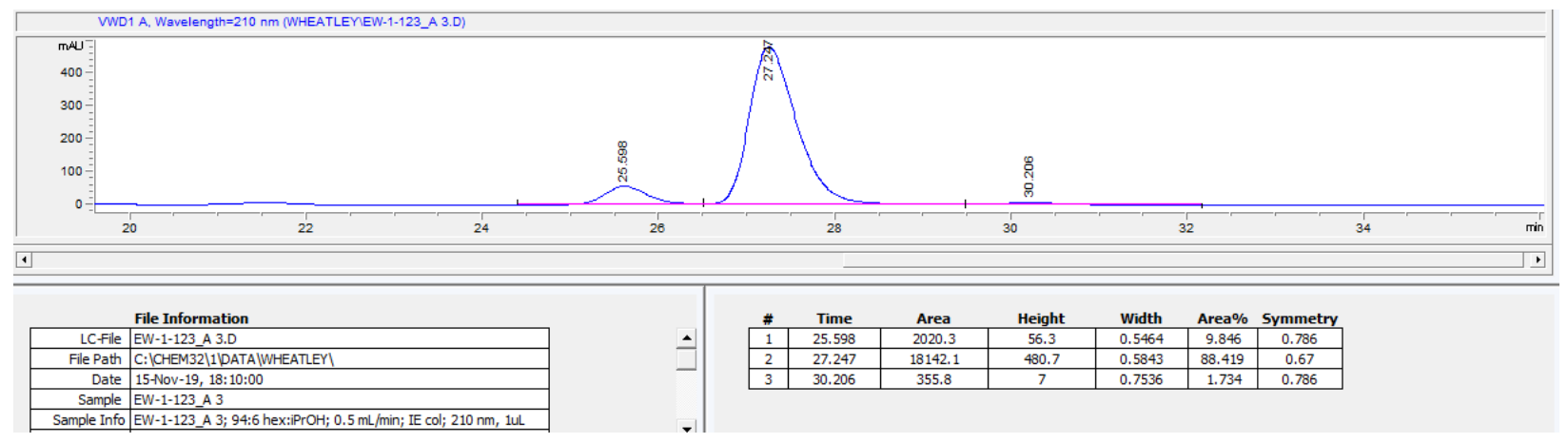

(1S,2S,E)-1-(benzo[b]thiophen-2-yl)-2-cyclopropyl-2-methyl-4-(4,4,5,5-tetramethyl-1,3,2-dioxaborolan-2yl)but-3-en-1-ol (6e)

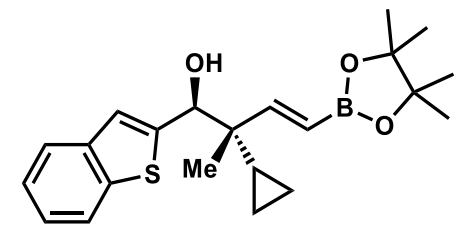

(6e)

Prepared according to General Method III using diboron 2e. Purified by silica gel column chromatography (Hexanes/Ethyl Acetate 25:1, 20:1, 15:1, 10:1, 8:1, 5:1) to give the product (17.6 mg, 92\% yield, 99:1 er, 7:1 dr) as a white solid (melting point $=182-185{ }^{\circ} \mathrm{C}$ ). Product could be recrystallized to 20:1 dr from EtOAc/hexanes. ${ }^{1} \mathbf{H}$ NMR $\left(600 \mathrm{MHz}, \mathrm{CDCl}_{3}\right) \delta 7.84-7.80(\mathrm{~d}, 8.0 \mathrm{~Hz}$, $1 \mathrm{H}), 7.76-7.71(\mathrm{~d}, 7.9 \mathrm{~Hz}, 1 \mathrm{H}), 7.37-7.27(\mathrm{~m}, 3 \mathrm{H}), 6.67-6.60(\mathrm{~d}, 18.4 \mathrm{~Hz}, 1 \mathrm{H}), 5.66-5.60(\mathrm{~d}, 18.3$ $\mathrm{Hz}, 1 \mathrm{H}), 5.02(\mathrm{~s}, 1 \mathrm{H}), 2.41(\mathrm{~s}, 1 \mathrm{H}), 1.31(\mathrm{~s}, 12 \mathrm{H}), 0.99(\mathrm{~s}, 3 \mathrm{H}), 0.49-0.42(\mathrm{~m}, 1 \mathrm{H}), 0.40-0.31(\mathrm{~m}, 2 \mathrm{H})$, $0.20-0.12(\mathrm{~m}, 1 \mathrm{H}) .{ }^{13} \mathrm{C}$ NMR $\left(151 \mathrm{MHz}, \mathrm{CDCl}_{3}\right) \delta$ 155.5, 145.3, 139.6, 139.3, 124.1, 124.0, 123.4, 122.2, $122.2,83.4,77.8,46.4,24.9,24.9,17.6,16.7,0.7,0.5$. HRMS (m/z) calc. for $\left[\mathrm{M}+\mathrm{Na}^{+}\right] \mathrm{C}_{22} \mathrm{H}_{29} \mathrm{BO}_{3} \mathrm{SNa}^{+}$: 407.1829 found: 407.1818. IR ( $\left.\mathrm{cm}^{-1}\right) 3447$ (m), 2978 (m), 1633 (m), 1349 (s), 1145 (s), 850 (w), 746 $(\mathrm{w}) \cdot[\alpha]_{\mathrm{D}}^{23}=-13.8\left(\mathrm{c}=0.88, \mathrm{CH}_{2} \mathrm{Cl}_{2}, \mathrm{l}=1 \mathrm{dm}\right)$.

6e was recrystallized from hexanes/EtOAc by vapor diffusion to $>20: 1 \mathrm{dr}$ and single crystal X-ray crystallography was used to determine the relative and absolute stereochemistry.

Diacel CHIRALPAK IC Column: 90:10 CO $2: \mathrm{PrOH} ; 1.0 \mathrm{~mL} / \mathrm{min} ; 210 \mathrm{~nm}$.

Racemic material: 


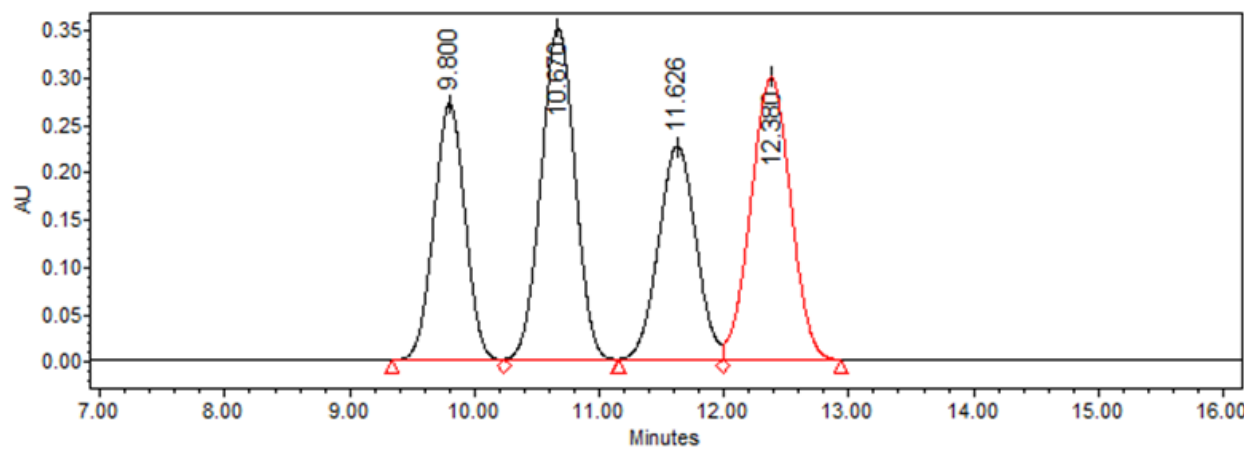

\begin{tabular}{|r|c|c|c|}
\hline $\begin{array}{c}\text { Retention } \\
\text { Time } \\
(\mathrm{min})\end{array}$ & $\begin{array}{c}\text { Area } \\
\left(\mu \mathrm{V}^{*} \mathrm{sec}\right)\end{array}$ & $\%$ Area & $\begin{array}{c}\text { Height } \\
(\mu \mathrm{V})\end{array}$ \\
\hline 9.800 & 4708677 & 20.82 & 270690 \\
\hline 10.670 & 6602301 & 29.19 & 349173 \\
\hline 11.626 & 4690324 & 20.74 & 225176 \\
\hline 12.380 & 6617031 & 29.26 & 298231 \\
\hline
\end{tabular}

Enantioenriched material: 99:1 er

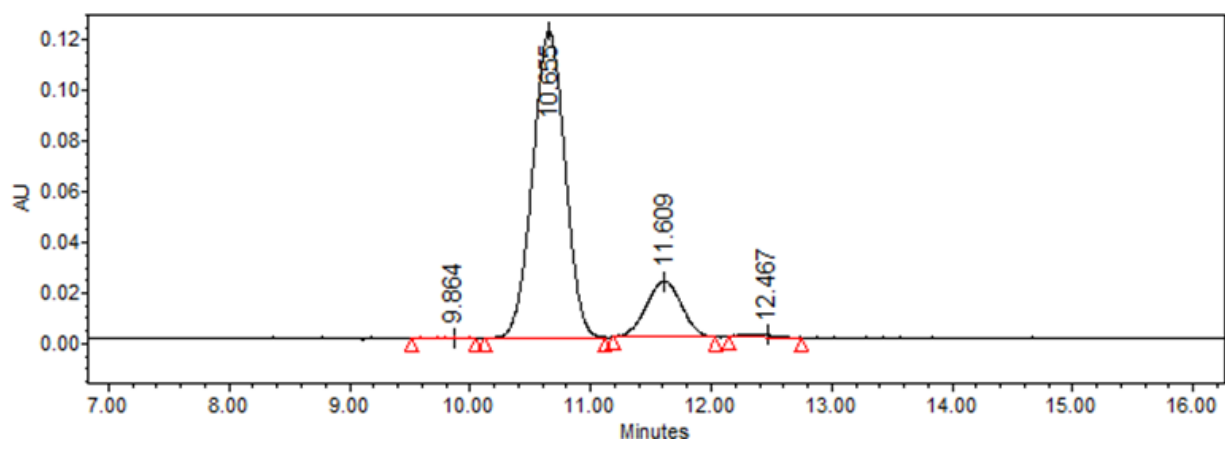

\begin{tabular}{|r|r|r|r|}
\hline $\begin{array}{c}\text { Retention } \\
\text { Time } \\
(\mathrm{min})\end{array}$ & $\begin{array}{c}\text { Area } \\
\left(\mu \mathrm{V}^{*} \mathrm{sec}\right)\end{array}$ & $\%$ Area & $\begin{array}{c}\text { Height } \\
(\mu \mathrm{V})\end{array}$ \\
\hline 9.864 & 3397 & 0.12 & 554 \\
\hline 10.655 & 2261340 & 82.92 & 120932 \\
\hline 11.609 & 444514 & 16.30 & 22127 \\
\hline 12.467 & 17832 & 0.65 & 1174 \\
\hline
\end{tabular}

tert-butyl-(S,E)-3-(1-hydroxy-2,2-dimethyl-4-(4,4,5,5-tetramethyl-1,3,2-dioxaborolan-2-yl)but-3-en-1-yl)$1 \mathrm{H}$-indole-1-carboxylate (6f)

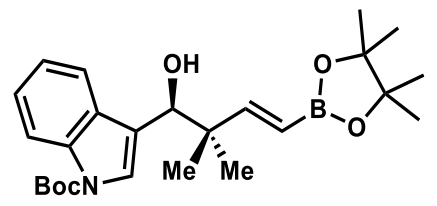

(6f)

Prepared according to General Method III using diboron 2f. Purified by silica gel column chromatography (Hexanes/Ethyl Acetate 25:1, 20:1, 15:1, 10:1) to give the product (18.6 mg, $84 \%$ yield, 96.5:3.5 er) as a colorless oil. ${ }^{1} \mathbf{H}$ NMR $\left(600 \mathrm{MHz}, \mathrm{CDCl}_{3}\right) \delta 8.18-8.07$ (d, $\left.8.4 \mathrm{~Hz}, 1 \mathrm{H}\right), 7.64-$ $7.58(\mathrm{~d}, 7.8 \mathrm{~Hz}, 1 \mathrm{H}), 7.52(\mathrm{~s}, 1 \mathrm{H}), 7.32-7.27(\mathrm{~m}, 1 \mathrm{H}), 7.24-7.15(\mathrm{t}, 7.5 \mathrm{~Hz}, 1 \mathrm{H}), 6.84-6.75(\mathrm{~d}, 18.4$ $\mathrm{Hz}, 1 \mathrm{H}), 5.61-5.51(\mathrm{~d}, 18.4 \mathrm{~Hz}, 1 \mathrm{H}), 4.84(\mathrm{~s}, 1 \mathrm{H}), 1.97$ (s, 1H), 1.67 (s, 9H), $1.30-1.25$ (d, $2.1 \mathrm{~Hz}$, 12H), 1.11 (s, 3H), 1.07 (s, 3H). ${ }^{13} \mathrm{C}$ NMR (151 MHz, $\left.\mathrm{CDCl}_{3}\right) \delta$ 160.2, 130.1, 124.3, 124.2, 122.5, 121.0, $120.8,115.2,83.8,83.3,77.4,74.4,44.3,28.3,25.0,24.9,24.3,21.1$. HRMS (m/z) calc. for $\left[{\left.\mathrm{M}-\mathrm{H}_{2} \mathrm{O}^{+}\right]}^{2}\right.$ $\mathrm{C}_{25} \mathrm{H}_{35} \mathrm{BNO}_{4}^{+}$: 424.2654 found: 424.2653. IR $\left(\mathrm{cm}^{-1}\right) 3451(\mathrm{w}), 2978(\mathrm{w}), 1734(\mathrm{~m}), 1454(\mathrm{~m}), 1350(\mathrm{~m})$, $1265(\mathrm{~s}), 1158(\mathrm{~m}), 740(\mathrm{~s}) .[\alpha]_{\mathrm{D}^{23}}=+0.5\left(\mathrm{c}=0.93, \mathrm{CH}_{2} \mathrm{Cl}_{2}, \mathrm{l}=1 \mathrm{dm}\right)$.

Phenomenex lux Cellulose 1-OD Column: 95:5 $\mathrm{CO}_{2}: \mathrm{iPrOH} ; 1.0 \mathrm{~mL} / \mathrm{min} ; 220 \mathrm{~nm}$. Racemic material: 


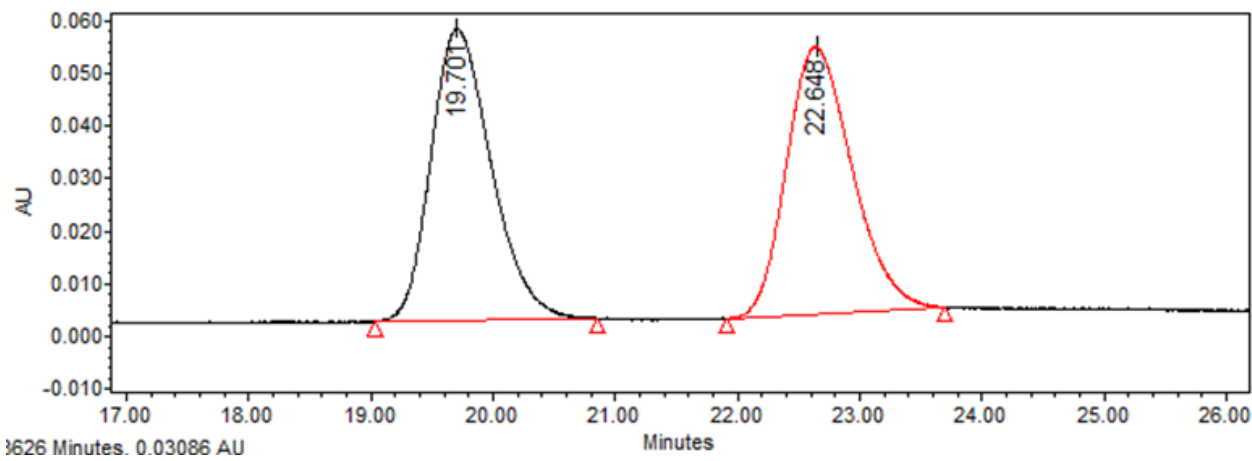

\begin{tabular}{c|c|c|c|}
\hline $\begin{array}{c}\text { Retention } \\
\text { Time } \\
(\mathrm{min})\end{array}$ & $\begin{array}{c}\text { Area } \\
\left(\mu \mathrm{V}^{*} \mathrm{sec}\right)\end{array}$ & $\%$ Area & $\begin{array}{c}\text { Height } \\
(\mu \mathrm{V})\end{array}$ \\
\hline 19.701 & 1904260 & 50.05 & 55436 \\
\hline 22.648 & 1900229 & 49.95 & 50884 \\
\hline
\end{tabular}

Enantioenriched material: 96.5:3.5 er

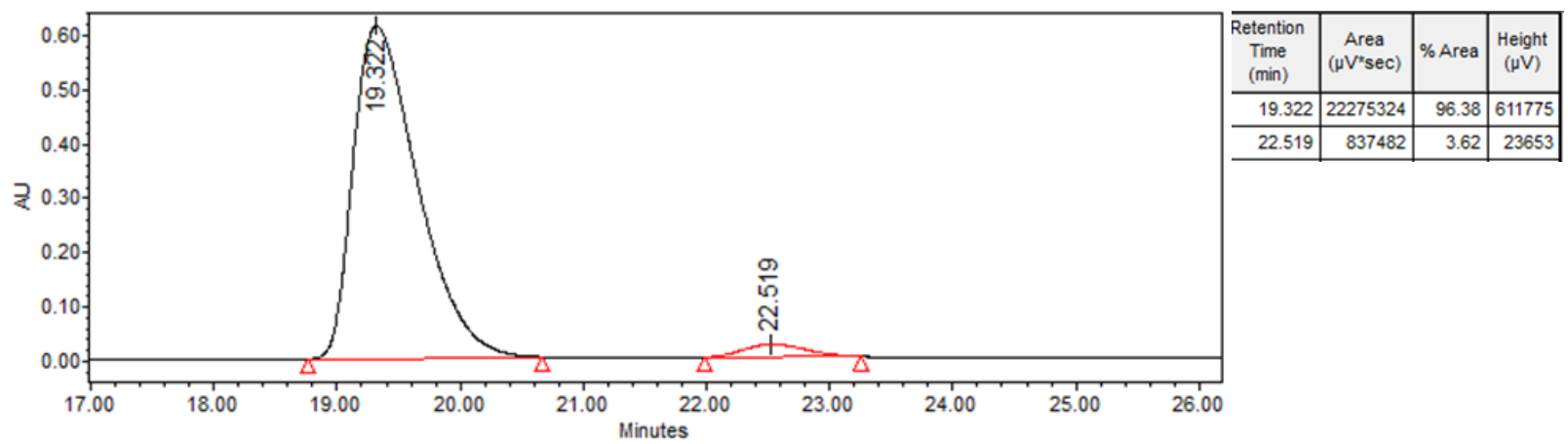

(1R,2S,E)-1-(cyclohex-1-en-1-yl)-2-methyl-2-phenethyl-4-(4,4,5,5-tetramethyl-1,3,2-dioxaborolan-2yl)but-3-en-1-ol (6g)<smiles>CC1(C)OB(/C=C/[C@@](C)(CCc2ccccc2)[C@H](O)C2=CCCCC2)OC1(C)C</smiles>

(6g)

Prepared according to General Method III using diboron 2g. Purified by silica gel column chromatography (Hexanes/Ethyl Acetate 25:1, 20:1, 15:1, 10:1) to give the product (16.6 mg, $84 \%$ yield, 96.5:3.5 er, 4:1 dr) as a colorless oil.

\section{Major Diastereomer}

${ }^{1} \mathrm{H}$ NMR $\left(600 \mathrm{MHz}, \mathrm{CDCl}_{3}\right) \delta 7.29$ - $7.24(\mathrm{~m}, 2 \mathrm{H}), 7.19-714(\mathrm{~m}, 3 \mathrm{H}), 6.70-6.65(\mathrm{~d}, 18.5 \mathrm{~Hz}, 1 \mathrm{H})$, $5.63(\mathrm{~s}, 1 \mathrm{H}), 5.52-5.48(\mathrm{~d}, 18.6 \mathrm{~Hz}, 1 \mathrm{H}), 3.81(\mathrm{~s}, 1 \mathrm{H}), 2.58-2.41(\mathrm{~m}, 2 \mathrm{H}), 2.07-2.01(\mathrm{~m}, 2 \mathrm{H}), 1.89$ (br s, 1H), $1.85-1.77(\mathrm{~m}, 1 \mathrm{H}), 1.77-1.70(\mathrm{~m}, 1 \mathrm{H}), 1.69-1.56(\mathrm{~m}, 4 \mathrm{H}), 1.55-1.46(\mathrm{~m}, 2 \mathrm{H}), 1.29(\mathrm{~s}$, 12H), 1.13 (s, 3H). ${ }^{13} \mathrm{C}$ NMR $\left(151 \mathrm{MHz}, \mathrm{CDCl}_{3}\right) \delta$ 159.0, 143.3, 138.5, 128.5, 128.4, 125.7, 125.6, 83.3, $82.5,46.8,39.4,30.9,27.3,25.2,25.0,24.8,22.9,22.7,18.8$.

\section{Minor Diastereomer}


${ }^{1} \mathrm{H}$ NMR $\left(600 \mathrm{MHz}, \mathrm{CDCl}_{3}\right) \delta 7.29$ - $7.24(\mathrm{~m}, 2 \mathrm{H}), 7.19-7.14(\mathrm{~m}, 3 \mathrm{H}), 6.75-6.70(\mathrm{~d}, 18.5 \mathrm{~Hz}, 1 \mathrm{H})$, $5.63(\mathrm{~s}, 1 \mathrm{H}), 5.56-5.51(\mathrm{~d}, 18.4 \mathrm{~Hz}, 1 \mathrm{H}), 3.81(\mathrm{~s}, 1 \mathrm{H}), 2.58-2.41(\mathrm{~m}, 2 \mathrm{H}), 2.01-1.98(\mathrm{~m}, 2 \mathrm{H}), 1.87$ (br s, 1H), $1.85-1.77(\mathrm{~m}, 1 \mathrm{H}), 1.77-1.70(\mathrm{~m}, 1 \mathrm{H}), 1.69-1.56(\mathrm{~m}, 4 \mathrm{H}), 1.55-1.46(\mathrm{~m}, 2 \mathrm{H}), 1.28(\mathrm{~s}$, 12H), 1.08 (s, 3H). ${ }^{13} \mathrm{C}$ NMR (151 MHz, $\left.\mathrm{CDCl}_{3}\right) \delta$ 159.6, 143.2, 137.8, 83.3, 47.1, 39.7, 30.8, 26.6, 25.2, $24.9,23.0,22.7,18.0$ (missing peaks overlap with other diastereomer).

HRMS (m/z) calc. for [M- $\left.\mathrm{H}_{2} \mathrm{O}^{+}\right] \mathrm{C}_{25} \mathrm{H}_{36} \mathrm{BO}_{2}{ }^{+}: 379.2803$ found: 379.2803. IR ( $\left.\mathrm{cm}^{-1}\right) 3487(\mathrm{w}), 2977(\mathrm{~m})$, 2930 (m), 1632 (m), $1389(\mathrm{~m}), 1351$ (s), 1146 (s), $970(\mathrm{w}), 851(\mathrm{w}) .[\alpha]_{\mathrm{D}^{23}}=-33.9$ (c = 0.83, $\mathrm{CH}_{2} \mathrm{Cl}_{2}, 1$ $=1 \mathrm{dm})$.

Diacel CHIRALPAK IE Column: $98: 2$ hex: $\mathrm{iPrOH} ; 0.35 \mathrm{~mL} / \mathrm{min} ; 210 \mathrm{~nm}$.

Racemic material:

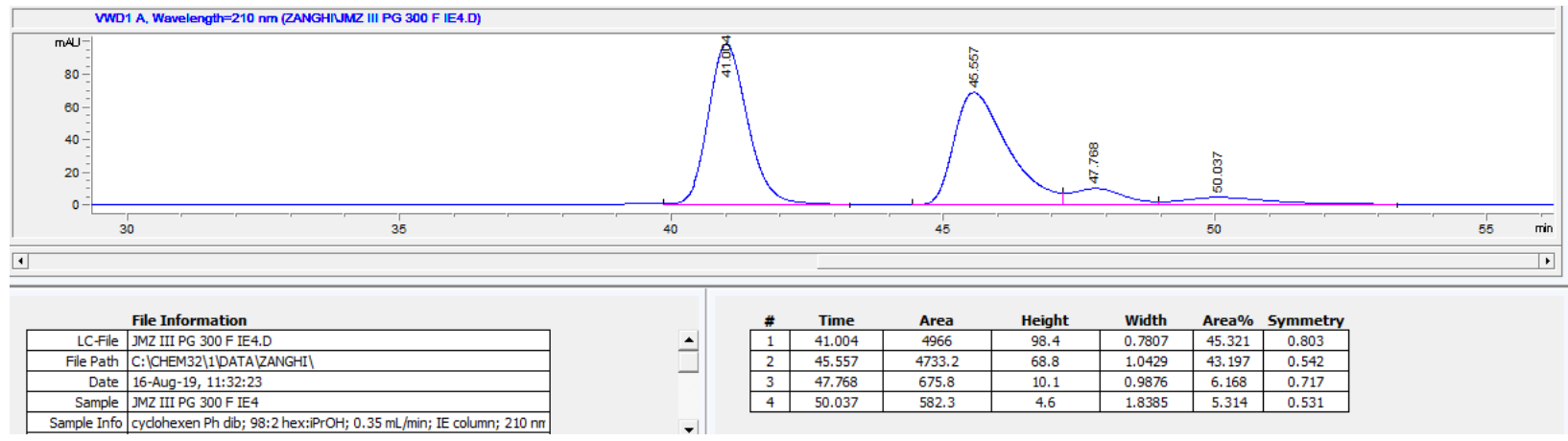

\section{Enantioenriched material:}

Major: 96.5:3.5 er

Minor: 90:10 er

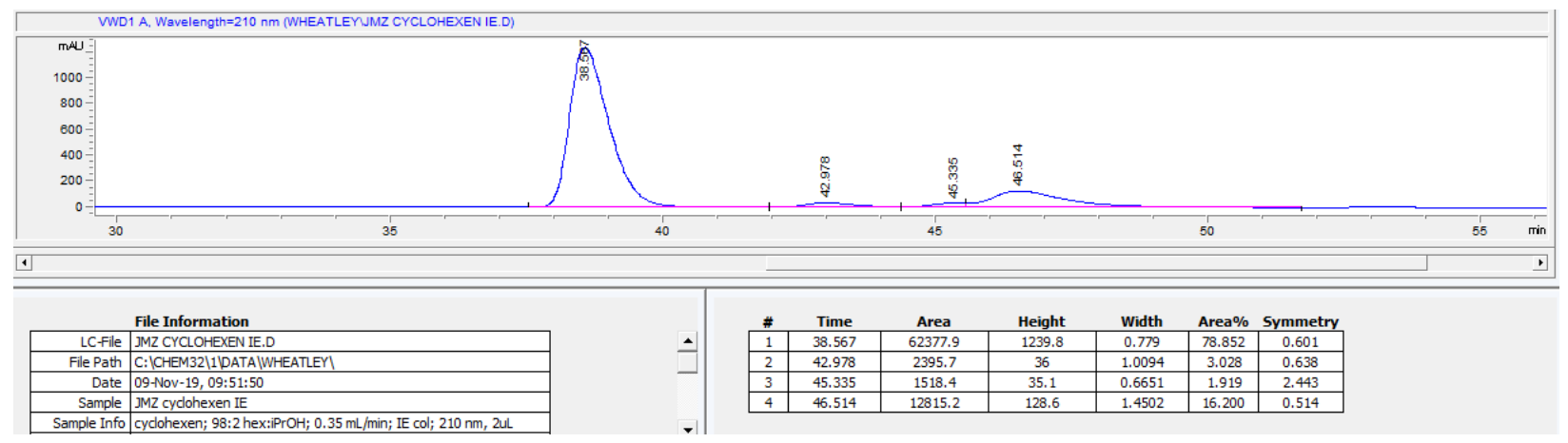

tert-butyl 4-((1R,2S,E)-2-(2-((tert-butyldimethylsilyl)oxy)ethyl)-1-hydroxy-2-methyl-4-(4,4,5,5-tetramethyl1,3,2-dioxaborolan-2-yl)but-3-en-1-yl)piperidine-1-carboxylate (6h)<smiles>CC(C)(C)OC(=O)N1CCC([C@H](O)[C@@](C)(/C=C/B2OC(C)(C)C(C)(C)O2)CC[Sb](C)(=O)=O)CC1</smiles>

(6h) 
Prepared according to General Method III using diboron $\mathbf{2 h}$. Purified by silica gel column chromatography (Hexanes/Ethyl Acetate 25:1, 20:1, 15:1, 10:1) to give the product $(19.2 \mathrm{mg}, 69 \%$ yield, 98.5:1.5 er, 6:1 dr) as a colorless oil (yield was adjusted to take aldehyde impurity into account). ${ }^{1} \mathbf{H}$ NMR (600 MHz, $\left.\mathrm{CDCl}_{3}\right) \delta 6.70-6.62(\mathrm{~d}, 18.6 \mathrm{~Hz}, 1 \mathrm{H}), 5.47$ - 5.39 (d, $\left.18.6 \mathrm{~Hz}\right), 4.20$ 3.89 (br s, 2H), $3.75-3.67(\mathrm{~m}, 1 \mathrm{H}), 3.66-3.57(\mathrm{~m}, 1 \mathrm{H}), 3.48-3.38(\mathrm{~m}, 1 \mathrm{H}), 2.73-2.51$ (br s, 2H), $1.96-1.82(\mathrm{~m}, 2 \mathrm{H}), 1.72(\mathrm{~s}, 1 \mathrm{H}), 1.58-1.49(\mathrm{~m}, 2 \mathrm{H}), 1.43(\mathrm{~s}, 9 \mathrm{H}), 1.40-1.35(\mathrm{~m}, 1 \mathrm{H}), 1.28-1.25(\mathrm{~d}$, $1.8 \mathrm{~Hz}, 12 \mathrm{H}), 1.05(\mathrm{~s}, 3 \mathrm{H}), 0.88(\mathrm{~s}, 9 \mathrm{H}), 0.07(\mathrm{~s}, 3 \mathrm{H}), 0.06(\mathrm{~s}, 3 \mathrm{H}) .{ }^{13} \mathrm{C}$ NMR $\left(151 \mathrm{MHz}, \mathrm{CDCl}_{3}\right) \delta$ 160.2, 154.9, 83.3, 79.2, 59.9, 46.2, 38.7, 28.6, 25.9, 25.0, 24.8, 19.4, 18.2, -5.4, -5.5. HRMS (m/z) calc. for $\left[\mathrm{M}+\mathrm{H}^{+}\right] \mathrm{C}_{29} \mathrm{H}_{57} \mathrm{BNO}_{6} \mathrm{Si}^{+}: 554.4043$ found: 554.4044. IR $\left(\mathrm{cm}^{-1}\right) 2930$ (m), 1696 (s), 1351 (m), 1169 (m), $1092(\mathrm{~m}), 851(\mathrm{w}), 776(\mathrm{w}) .[\alpha]_{\mathrm{D}^{23}}=-7.6\left(\mathrm{c}=0.99, \mathrm{CH}_{2} \mathrm{Cl}_{2}, \mathrm{l}=1 \mathrm{dm}\right)$.

Phenomenex lux Amylose 1-AD Column: $93: 7 \mathrm{CO}_{2}: \mathrm{PrOH} ; 1.0 \mathrm{~mL} / \mathrm{min} ; 210 \mathrm{~nm}$.

\section{Racemic material:}

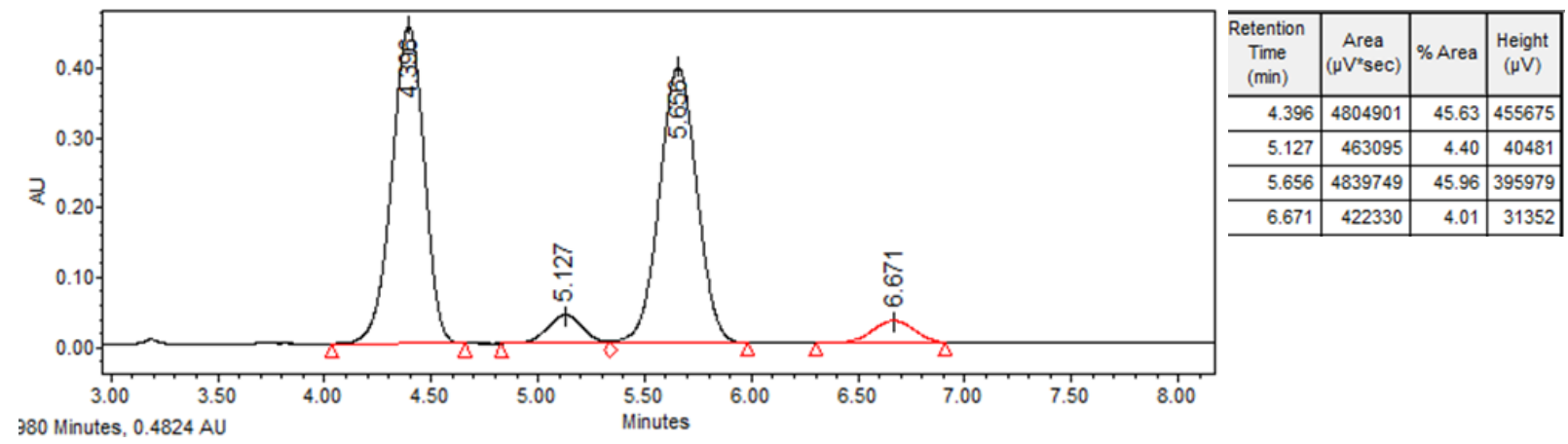

Enantioenriched material: 98.5:1.5 er

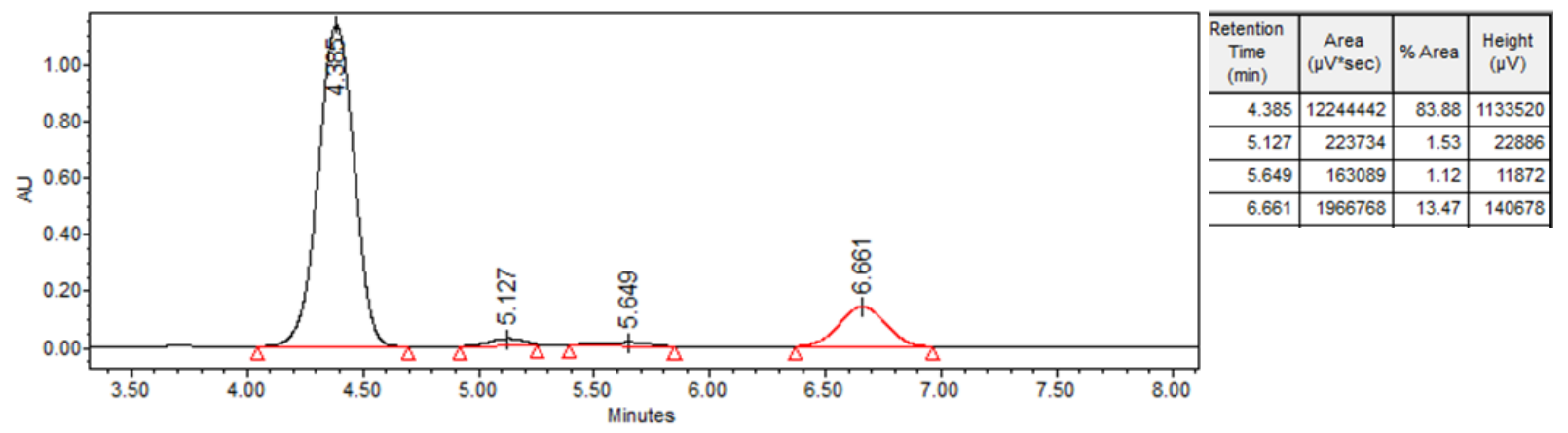

(1S,2S)-2-methyl-1-phenyl-2-vinylhexan-1-ol (8) 


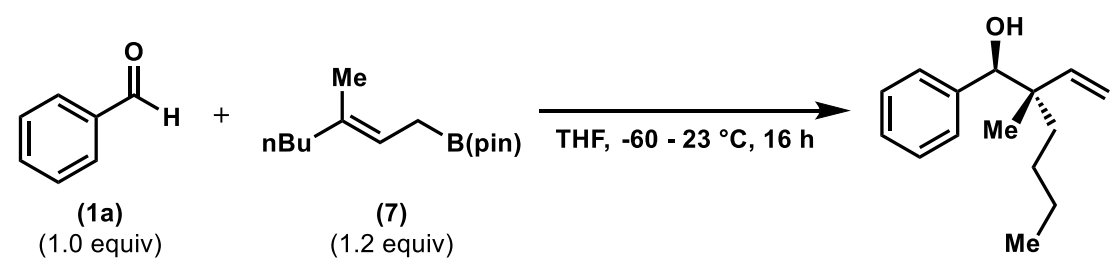

(8)

In a $\mathrm{N}_{2}$-filled glovebox, boronate 7 (14 mg, $0.06 \mathrm{mmol}, 1.2$ eq.) was weighed into an $8 \mathrm{~mL}$ vial and THF $(300 \mu \mathrm{L})$ and magnetic stirrer bar were added. In a second $8 \mathrm{~mL}$ vial, a stock solution of benzaldehyde 1a (0.50 M in THF) was prepared. Both vials were capped with a Teflon-lined lid, sealed with electrical tape, and removed from the glovebox. Both vials were cooled to $-78{ }^{\circ} \mathrm{C}$ (dry ice/acetone bath) and $50 \mu \mathrm{L}$ aldehyde stock solution $(0.050 \mathrm{mmol}, 1.0$ equiv) was added to the boronate. The reaction was taped over with Teflon and electrical tape and allowed to sit in a -60 ${ }^{\circ} \mathrm{C}$ cryobath for 12 hours.

Upon completion, the reaction was quenched at $-60{ }^{\circ} \mathrm{C}$ with $1 \mathrm{~mL}$ saturated $\mathrm{NH}_{4} \mathrm{Cl}$ (aq) and stirred vigorously at $22^{\circ} \mathrm{C}$ for 30 minutes. Diethyl ether was added and the layers separated. The aqueous layer was extracted three times with diethyl ether $(3 \times 1 \mathrm{~mL})$ and the combined organic extracts were dried over $\mathrm{MgSO}_{4}$, filtered and concentrated in vacuo.

Homoallylic alcohol 8 was purified by silica gel column chromatography (Hexanes/Ethyl Acetate 25:1, 20:1, 15:1, 10:1) to give the product (7.2 mg, 66\% yield, 9:1 dr, 50:50 er) as a colorless

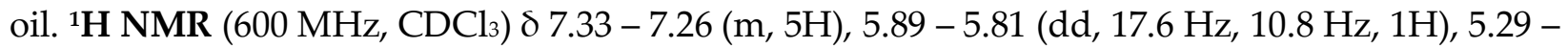
$5.24(\mathrm{dd}, 10.8,1.3 \mathrm{~Hz}, 1 \mathrm{H}), 5.08(\mathrm{dd}, 17.6,1.4 \mathrm{~Hz}, 1 \mathrm{H}), 4.43(\mathrm{~d}, 2.0 \mathrm{~Hz}, 1 \mathrm{H}), 2.03(\mathrm{~d}, 2.4 \mathrm{~Hz}, 1 \mathrm{H})$, $1.43-1.35(\mathrm{~m}, 1 \mathrm{H}), 1.28-1.23(\mathrm{~m}, 3 \mathrm{H}), 1.19-1.13(\mathrm{~m}, 2 \mathrm{H}), 0.89(\mathrm{~s}, 3 \mathrm{H}), 0.88-0.84(\mathrm{t}, 7.3 \mathrm{~Hz}, 3 \mathrm{H})$. ${ }^{13} \mathrm{C}$ NMR $\left(151 \mathrm{MHz}, \mathrm{CDCl}_{3}\right) \delta 144.3,140.7,128.2,127.6,127.6,115.9,80.2,46.0,37.5,26.4,23.6,16.4$, 14.3. HRMS (m/z) calc. for [M-H2O $\left.\mathrm{O}^{+}\right] \mathrm{C}_{15} \mathrm{H}_{21}{ }^{+}: 201.1638$ found: 201.1633. IR (cm-1) 3445 (br), 2956 (s), 2930 (s), 2860 (m), 1455 (w), 1025 (m), 912 (m), 702 (s).

Diacel CHIRALPAK IC Column: 95:5 $\mathrm{CO}_{2}: \mathrm{iPrOH} ; 1.0 \mathrm{~mL} / \mathrm{min} ; 220 \mathrm{~nm}$.

Racemic material:

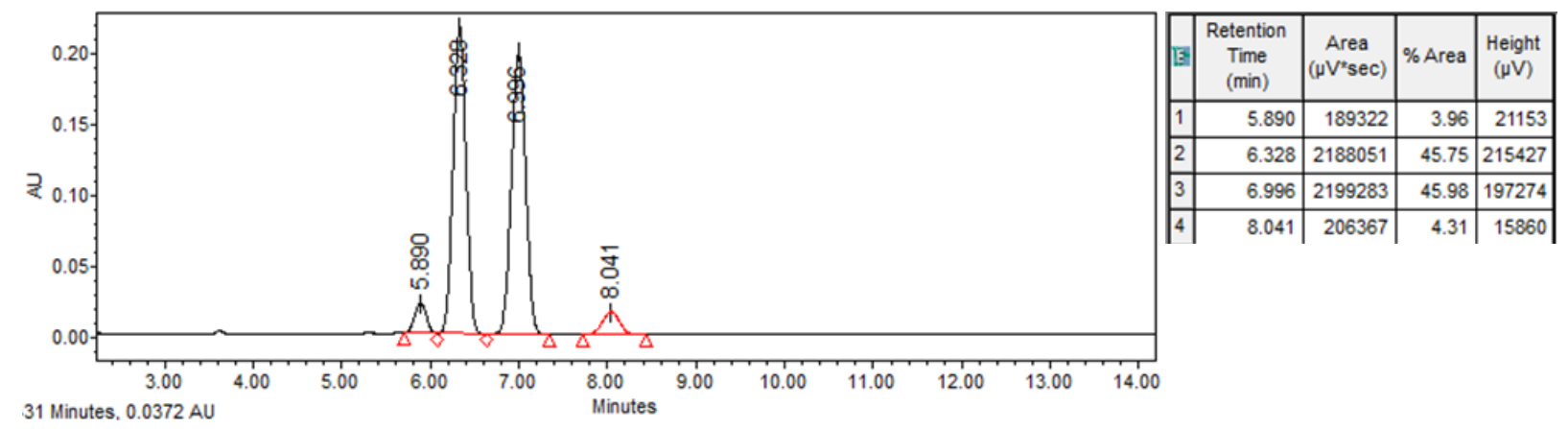

\section{Enantioenriched material:}




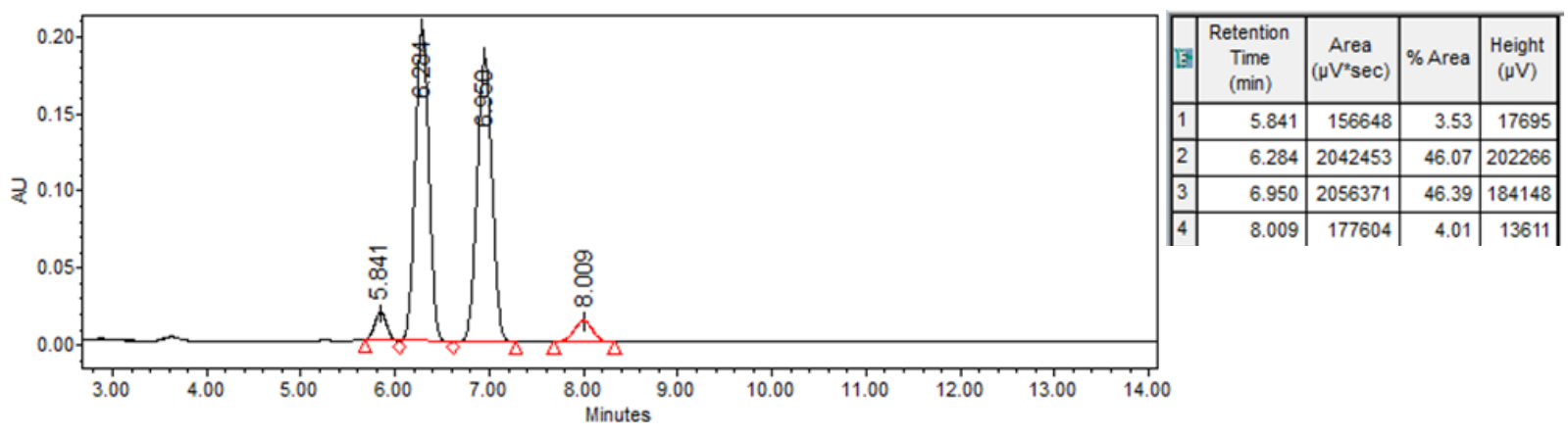

(S,E)-2-methyl-1-phenyl-2-(2-(4,4,5,5-tetramethyl-1,3,2-dioxaborolan-2-yl)vinyl)hexan-1-one (9)

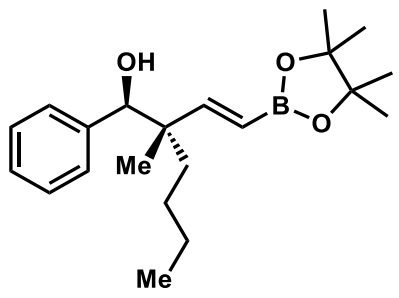

(3a)

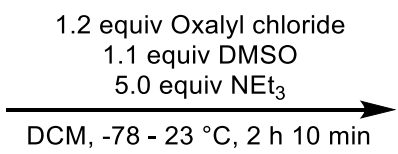

DCM, $-78-23^{\circ} \mathrm{C}, 2 \mathrm{~h} 10 \mathrm{~min}$

To an oven-dried $8 \mathrm{~mL}$ vial, oxalyl chloride $(10 \mu \mathrm{L}, 0.12 \mathrm{mmol}, 1.2 \mathrm{eq}$. $)$ and DCM $(0.5 \mathrm{~mL})$ were added and cooled to $-78{ }^{\circ} \mathrm{C}$ in a dry ice/acetone bath. DMSO $(7.5 \mu \mathrm{L}, 0.11 \mathrm{mmol}, 1.1$ eq.) was added and the reaction mixture was allowed to stir at $-78{ }^{\circ} \mathrm{C}$ for 10 minutes. Substrate (3a) (32.9 $\mathrm{mg}, 0.10 \mathrm{mmol}, 1.0$ eq.) in DCM $(0.5 \mathrm{~mL})$ was added and the reaction allowed to stir at $-78{ }^{\circ} \mathrm{C}$ for an additional 30 minutes. Triethylamine $\left(66 \mu \mathrm{L}, 0.50 \mathrm{mmol}, 5.0\right.$ eq.) was added at $-78{ }^{\circ} \mathrm{C}$ and stirred for 30 minutes before allowing the reaction to warm to room temperature and stir for another hour. Upon completion, the reaction was diluted with DCM $(1 \mathrm{~mL})$, washed with 5\% $\mathrm{HCl}$, saturated $\mathrm{NaHCO}_{3}$ and brine. The organic layer was dried with $\mathrm{MgSO}_{4}$, filtered through cotton, concentrated under vacuum and purified by silica gel column chromatography (Hexanes/Ethyl Acetate 25:1, 20:1, 15:1, 10:1) to give ketone (9) (17.4 mg, 53 \% yield, 97.5:2.5 er) as a colorless oil.

${ }^{1} \mathbf{H}$ NMR $\left(600 \mathrm{MHz}, \mathrm{CDCl}_{3}\right) \delta 7.82-7.77(\mathrm{~m}, 2 \mathrm{H}), 7.47$ - $7.41(\mathrm{~m}, 1 \mathrm{H}), 7.38-7.32(\mathrm{~m}, 2 \mathrm{H}), 6.95$ $6.88(\mathrm{~d}, 18.4 \mathrm{~Hz}, 1 \mathrm{H}), 5.65-5.58(\mathrm{~d}, 18.4 \mathrm{~Hz}, 1 \mathrm{H}), 1.97-1.87(\mathrm{~m}, 1 \mathrm{H}), 1.78-1.69(\mathrm{~m}, 1 \mathrm{H}), 1.35(\mathrm{~s}$, $3 \mathrm{H}), 1.27(\mathrm{~s}, 12 \mathrm{H}), 1.24-1.17(\mathrm{~m}, 4 \mathrm{H}), 0.82-0.77$ (t, $7.0 \mathrm{~Hz}, 3 \mathrm{H}) .{ }^{13} \mathrm{C}$ NMR $\left(151 \mathrm{MHz}, \mathrm{CDCl}_{3}\right) \delta$ 204.3, 157.5, 137.6, 131.7, 129.1, 128.0, 83.5, 55.2, 38.5, 26.5, 24.9, 24.9, 23.4, 22.9, 14.0. HRMS (m/z) calc. for $\left[\mathrm{M}+\mathrm{H}^{+}\right] \mathrm{C}_{21} \mathrm{H}_{32} \mathrm{BO}_{3}^{+}$: 343.2439 found: 343.2442. IR $\left(\mathrm{cm}^{-1}\right) 2977(\mathrm{~m}), 2931(\mathrm{~m}), 2861(\mathrm{w}), 1681$ $(\mathrm{m}), 1625(\mathrm{~m}), 1372(\mathrm{~m}), 1351(\mathrm{~s}), 1213(\mathrm{~m}), 1145(\mathrm{~s}), 970(\mathrm{~m}), 850(\mathrm{w}) .[\alpha]_{\mathrm{D}^{23}}=-3.4\left(\mathrm{c}=0.87, \mathrm{CH}_{2} \mathrm{Cl}_{2}\right.$, $1=1 \mathrm{dm})$.

Diacel CHIRALPAK IA Column: 97:3 hexanes:iPrOH; $0.35 \mathrm{~mL} / \mathrm{min} ; 210 \mathrm{~nm}$.

Racemic material: 


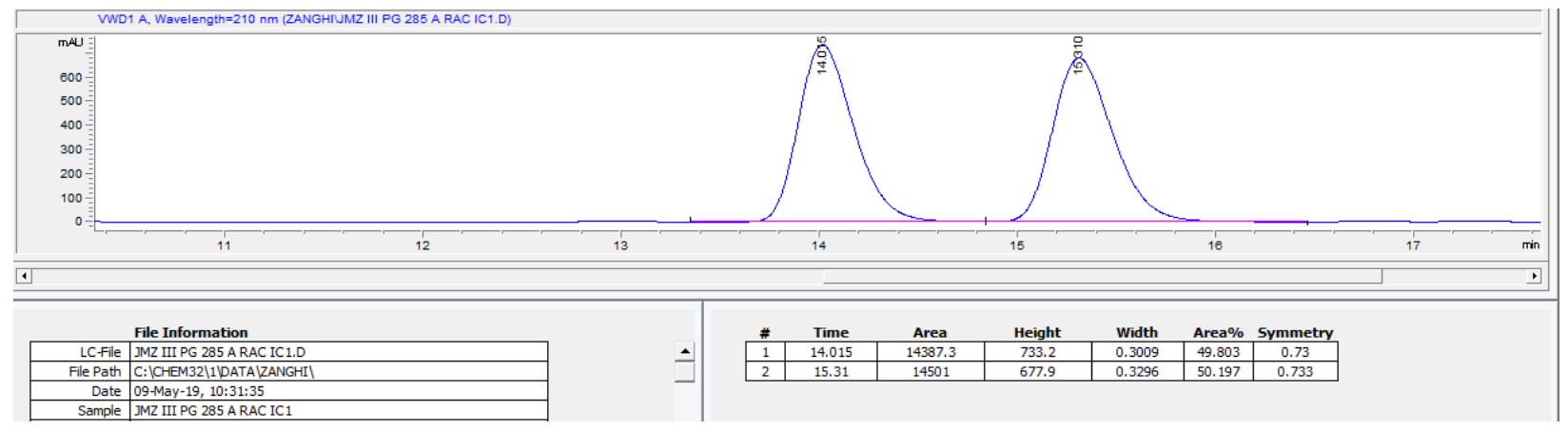

Enantioenriched material: 97.5:2.5 er

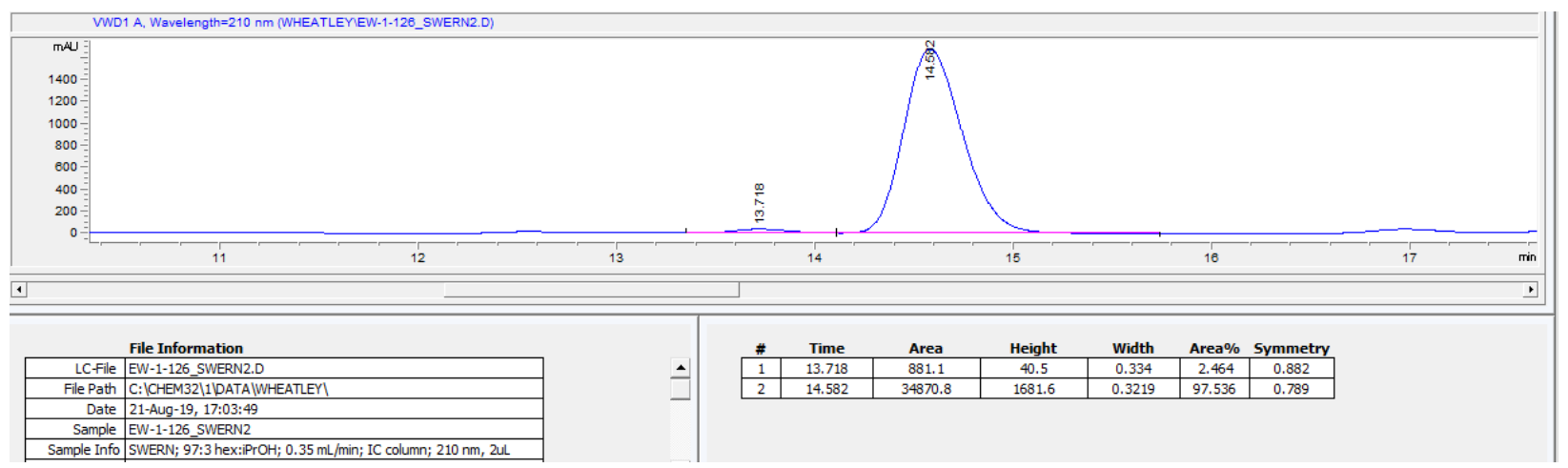

tert-butyl-((2S,3S,E)-3-hydroxy-4,4-dimethyl-6-(4,4,5,5-tetramethyl-1,3,2-dioxaborolan-2-yl)hex-5-en-2yl)carbamate (11)

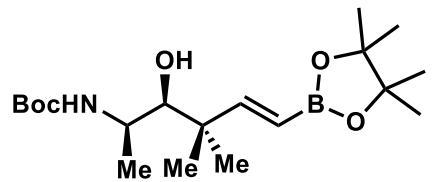

(11)

Prepared according to General Method III. Purified by silica gel column chromatography (Hexanes/Ethyl Acetate 25:1, 20:1, 15:1, 10:1) to give the product (16.8 mg, 91\% yield, 3.2:1 dr) as a colorless oil.

\section{Major Diastereomer}

${ }^{1} \mathbf{H}$ NMR $\left(600 \mathrm{MHz}, \mathrm{CDCl}_{3}\right) \delta 6.72-6.67(\mathrm{~d}, 18.4 \mathrm{~Hz}, 1 \mathrm{H}), 5.48-5.43(\mathrm{~d}, 18.4 \mathrm{~Hz}, 1 \mathrm{H}), 4.88-4.81$ (d, $8.3 \mathrm{~Hz}, 1 \mathrm{H}), 3.83-3.75(\mathrm{~m}, 1 \mathrm{H}), 2.13-2.08$ (br s, 1H), $1.71-1.68$ (br s, 1H), $1.43(\mathrm{~s}, 9 \mathrm{H}), 1.26(\mathrm{~s}$, 12H), 1.23 (s, 3H), 1.10 (s, 3H), 1.09 (s, 3H). $\left.{ }^{13} \mathrm{C} \mathrm{NMR} \mathrm{(151} \mathrm{MHz,} \mathrm{CDCl}\right) \delta$ 159.8, 155.4, 83.3, 80.8, 75.2, 48.1, 42.5, 28.6, 24.94, 24.92, 24.7, 23.3, 15.9.

\section{Minor Diastereomer}

${ }^{1} \mathrm{H}$ NMR $\left(600 \mathrm{MHz}, \mathrm{CDCl}_{3}\right) \delta 6.61-6.57$ (d, $\left.18.4 \mathrm{~Hz}, 1 \mathrm{H}\right), 5.49-5.45(\mathrm{~d}, 18.4 \mathrm{~Hz}, 1 \mathrm{H}), 4.92-4.89$ $(\mathrm{d}, 10.3 \mathrm{~Hz}, 1 \mathrm{H}), 3.94-3.88(\mathrm{~m}, 1 \mathrm{H}), 2.07-2.05$ (br s, 1H), $2.00-1.97$ (br s, 1H), 1.43 (s, 9H), 1.26 (s, 12H), 123 (s, 3H), 1.06 (s, 3H), 1.059 (s, 3H). $\left.{ }^{13} \mathrm{C} \mathrm{NMR} \mathrm{(151} \mathrm{MHz,} \mathrm{CDCl}_{3}\right) \delta$ 160.1, 155.5, 83.4, $79.9,79.4,45.8,43.2,28.6,25.0,24.97,23.8,21.6,15.4$. 
HRMS (m/z) calc. for $\left[\mathrm{M}+\mathrm{H}^{+}\right] \mathrm{C}_{19} \mathrm{H}_{37} \mathrm{BNO}_{5}{ }^{+}: 370.2759$ found: 370.2755. IR (cm $\left.{ }^{-1}\right) 3445$ (br), 2978 (s), $2931(\mathrm{~m}), 1688(\mathrm{~s}), 1633(\mathrm{~s}), 1350(\mathrm{~s}), 1052(\mathrm{w}) .[\alpha]_{\mathrm{D}^{23}}=+9.2\left(\mathrm{c}=0.84, \mathrm{CH}_{2} \mathrm{Cl}_{2}, 1=1 \mathrm{dm}\right)$.

tert-butyl-((2R,3S,4S)-3-hydroxy-4-methyl-4-((E)-2-(4,4,5,5-tetramethyl-1,3,2-dioxaborolan-2yl)vinyl)octan-2-yl)carbamate (12)

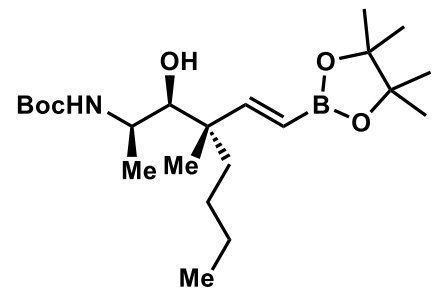

(12)

Prepared according to General Method III. Purified by silica gel column chromatography (Hexanes/Ethyl Acetate 20:1, 15:1, 10:1, 8:1, 5:1) to give the product (17.9 mg, 87\% yield, $3.8: 1 \mathrm{dr}$ ) as a colorless oil.

\section{Major Diastereomer}

${ }^{1} \mathbf{H}$ NMR $\left(600 \mathrm{MHz}, \mathrm{CDCl}_{3}\right) \delta 6.68$ - 6.58 (d, $\left.18.6 \mathrm{~Hz}, 1 \mathrm{H}\right), 5.46$ - 5.36 (d, $\left.18.5 \mathrm{~Hz}, 1 \mathrm{H}\right), 4.96$ - 4.90 $(\mathrm{d}, 8.7 \mathrm{~Hz}, 1 \mathrm{H}), 3.90-3.80(\mathrm{~m}, 1 \mathrm{H}), 3.49(\mathrm{~s}, 1 \mathrm{H}), 1.94(\mathrm{~s}, 1 \mathrm{H}), 1.42(\mathrm{~s}, 9 \mathrm{H}), 1.26(\mathrm{~s}, 15 \mathrm{H}), 1.22-1.07$ $(\mathrm{m}, 4 \mathrm{H}), 1.07-1.00(\mathrm{~m}, 5 \mathrm{H}), 0.89-0.82$ (t, $7.3 \mathrm{~Hz}, 3 \mathrm{H}) .{ }^{13} \mathrm{C} \mathrm{NMR}\left(151 \mathrm{MHz}, \mathrm{CDCl}_{3}\right) \delta$ 159.2, 155.3, 83.3, 79.8, 47.7, 45.6, 37.9, 28.6, 26.2, 24.9, 24.9, 23.5, 18.2, 15.8, 14.2.

\section{Minor Diastereomer}

${ }^{1} \mathrm{H}$ NMR $\left(600 \mathrm{MHz}, \mathrm{CDCl}_{3}\right) \delta 6.55$ - $6.46(\mathrm{~d}, 18.6 \mathrm{~Hz}, 1 \mathrm{H}), 5.46-5.37$ (d, $\left.18.5 \mathrm{~Hz}, 1 \mathrm{H}\right), 4.89$ - 4.85 $(\mathrm{d}, 9.4 \mathrm{~Hz}, 1 \mathrm{H}), 3.78-3.70(\mathrm{~m}, 1 \mathrm{H}), 3.25(\mathrm{~s}, 1 \mathrm{H}), 2.02(\mathrm{~s}, 1 \mathrm{H}), 1.42(\mathrm{~s}, 9 \mathrm{H}), 1.26(\mathrm{~s}, 15 \mathrm{H}), 1.22-1.07$ $(\mathrm{m}, 4 \mathrm{H}), 1.07-1.00(\mathrm{~m}, 5 \mathrm{H}), 0.89-0.82$ (t, $7.3 \mathrm{~Hz}, 3 \mathrm{H}) .{ }^{13} \mathrm{C}$ NMR $\left(151 \mathrm{MHz}, \mathrm{CDCl}_{3}\right) \delta$ 83.3, 79.3, $51.3,46.4,37.2,28.6,26.2,25.0,23.6,18.1$ (missing peaks overlap with other diastereomer).

HRMS (m/z) calc. for [M+H' $\mathrm{C}_{22} \mathrm{H}_{43} \mathrm{BNO}_{5}^{+}: 412.3229$ found: 412.3241. IR ( $\left.\mathrm{cm}^{-1}\right) 3445$ (br), $2978(\mathrm{~s})$, $2933(\mathrm{~m}), 1689(\mathrm{~s}), 1632(\mathrm{~m}), 1350(\mathrm{~s}), 1168(\mathrm{~m}), 1147(\mathrm{~m}), 977(\mathrm{w}), 851(\mathrm{w}) .[\alpha]_{\mathrm{D}^{23}}=+10.7(\mathrm{c}=0.90$, $\left.\mathrm{CH}_{2} \mathrm{Cl}_{2}, \mathrm{l}=1 \mathrm{dm}\right)$.

tert-butyl-((2S,3S,4S)-3-hydroxy-4-methyl-4-((E)-2-(4,4,5,5-tetramethyl-1,3,2-dioxaborolan-2yl)vinyl)octan-2-yl)carbamate (13)

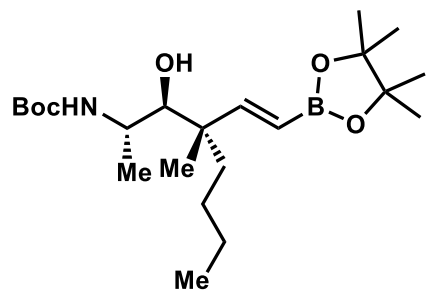

(13)

Prepared according to General Method III. Purified by silica gel column chromatography (Hexanes/Ethyl Acetate 20:1, 15:1, 10:1, 8:1, 5:1) to give the product (17.7 mg, 86\% yield, 1:1 dr) as 
a colorless oil. ${ }^{1} \mathrm{H}$ NMR $\left(600 \mathrm{MHz}, \mathrm{CDCl}_{3}\right) \delta 6.61-6.54(\mathrm{~d}, 18.3 \mathrm{~Hz}, 1 \mathrm{H}), 5.49-5.42(\mathrm{~d}, 18.4 \mathrm{~Hz}$, $1 \mathrm{H}), 4.93-4.85(\mathrm{~d}, 8.8 \mathrm{~Hz}, 1 \mathrm{H}), 3.86-3.68(\mathrm{~d}, 7.8 \mathrm{~Hz}, 1 \mathrm{H}), 3.48$ (br s, $1 \mathrm{H}), 2.18$ (br s, $1 \mathrm{H}), 1.42$ (s, $9 \mathrm{H}), 1.36-1.31(\mathrm{~m}, 2 \mathrm{H}), 1.27(\mathrm{~s}, 12 \mathrm{H}), 1.26-1.22(\mathrm{~m}, 2 \mathrm{H}), 1.22-1.17(\mathrm{~m}, 2 \mathrm{H}), 1.05(\mathrm{~s}, 3 \mathrm{H}), 0.89-$ $0.85(\mathrm{t}, 6.9 \mathrm{~Hz}, 3 \mathrm{H}) .{ }^{13} \mathrm{C} \mathrm{NMR}\left(151 \mathrm{MHz}, \mathrm{CDCl}_{3}\right) \delta$ 159.5, 158.6, 83.4, 80.5, 78.7, 48.3, 45.9, 37.5, 28.6, 26.3, 25.0, 23.6, 22.0, 16.4, 14.2.

Visible peaks for other diastereomer

${ }^{1}$ H NMR $\left(600 \mathrm{MHz}, \mathrm{CDCl}_{3}\right) \delta 6.53$ - 6.46 (d, $\left.18.5 \mathrm{~Hz}, 1 \mathrm{H}\right), 5.49$ - 5.42 (d, $\left.18.4 \mathrm{~Hz}, 1 \mathrm{H}\right), 5.03$ - 4.94 (d, $9.0 \mathrm{~Hz}, 1 \mathrm{H}), 4.08-3.92$ (d, $7.2 \mathrm{~Hz}, 1 \mathrm{H}), 3.24$ (br s, 1H), 1.85 (br s, 1H), 1.42 (s, 9H), 1.36 - 1.31 $(\mathrm{m}, 2 \mathrm{H}), 1.27(\mathrm{~s}, 12 \mathrm{H}), 1.26-1.22(\mathrm{~m}, 2 \mathrm{H}), 1.22-1.17(\mathrm{~m}, 2 \mathrm{H}), 0.98(\mathrm{~s}, 3 \mathrm{H}), 0.89-0.85(\mathrm{t}, 6.9 \mathrm{~Hz}$, 3H). ${ }^{13} \mathrm{C}$ NMR $\left(151 \mathrm{MHz}, \mathrm{CDCl}_{3}\right) \delta 83.3,46.6,45.1,37.8,28.6,26.3,25.0,23.6,19.5$ (missing peaks overlap with other diastereomer).

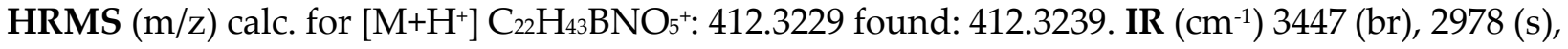
$2873(\mathrm{~m}), 1688(\mathrm{~s}), 1351$ (s), $1168(\mathrm{~m}), 970(\mathrm{w}), 851(\mathrm{w}) .[\alpha]_{\mathrm{D}^{23}}=-10.3\left(\mathrm{c}=1.01, \mathrm{CH}_{2} \mathrm{Cl}_{2}, \mathrm{l}=1 \mathrm{dm}\right)$.

(R,E)-2-(3-(4-methoxybenzyl)-3-methylhept-1-en-1-yl)-4,4,5,5-tetramethyl-1,3,2-dioxaborolane (14)

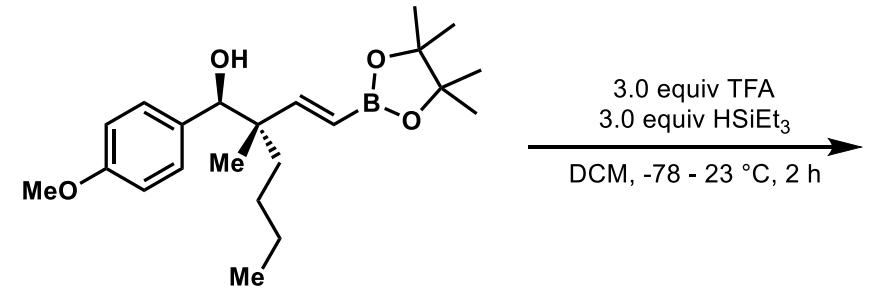

(3b)

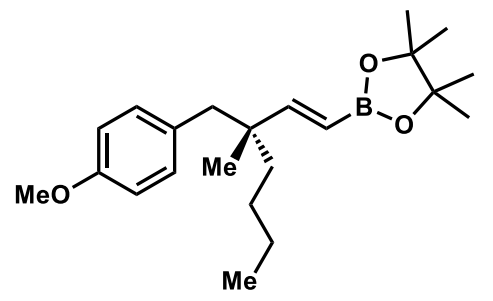

(14)

Based on a literature procedure ${ }^{6}$, to an $8 \mathrm{~mL}$ vial charged with magnetic stir bar, $3 \mathbf{b}(20.9 \mathrm{mg}$, $0.0558 \mathrm{mmol})$ was weighed and dry DCM $(500 \mu \mathrm{L})$ was added. The solution was then cooled to $78{ }^{\circ} \mathrm{C}$ and trifluoroacetic acid $(12.9 \mu \mathrm{L}, 0.167 \mathrm{mmol}, 3.0$ equiv) was added via microsyringe followed immediately by addition of triethylsilane $(27 \mu \mathrm{L}, 0.167 \mathrm{mmol}, 3.0$ equiv). After 10 minutes, the solution was allowed to warm to room temperature and stirred for 2 hours. Upon completion, the reaction was quenched with saturated $\mathrm{NaHCO}_{3}(1 \mathrm{~mL})$ and the aqueous layer extracted with $\mathrm{Et}_{2} \mathrm{O}(3 \times 1 \mathrm{~mL})$. The organic layers were combined, dried over $\mathrm{MgSO}_{4}$, filtered and concentrated by rotary evaporation. The crude material was purified by silica gel column chromatography (Hexanes/Ethyl Acetate 25:1, 20:1, 15:1, 10:1) to give the product (16.3 $\mathrm{mg}$, $82 \%$ yield, 98:2 er) as a colorless oil.

Diacel CHIRALPAK IC Column: 98.5:1.5 hexanes:iPrOH; $0.15 \mathrm{~mL} / \mathrm{min} ; 210 \mathrm{~nm}$.

Racemic material: 


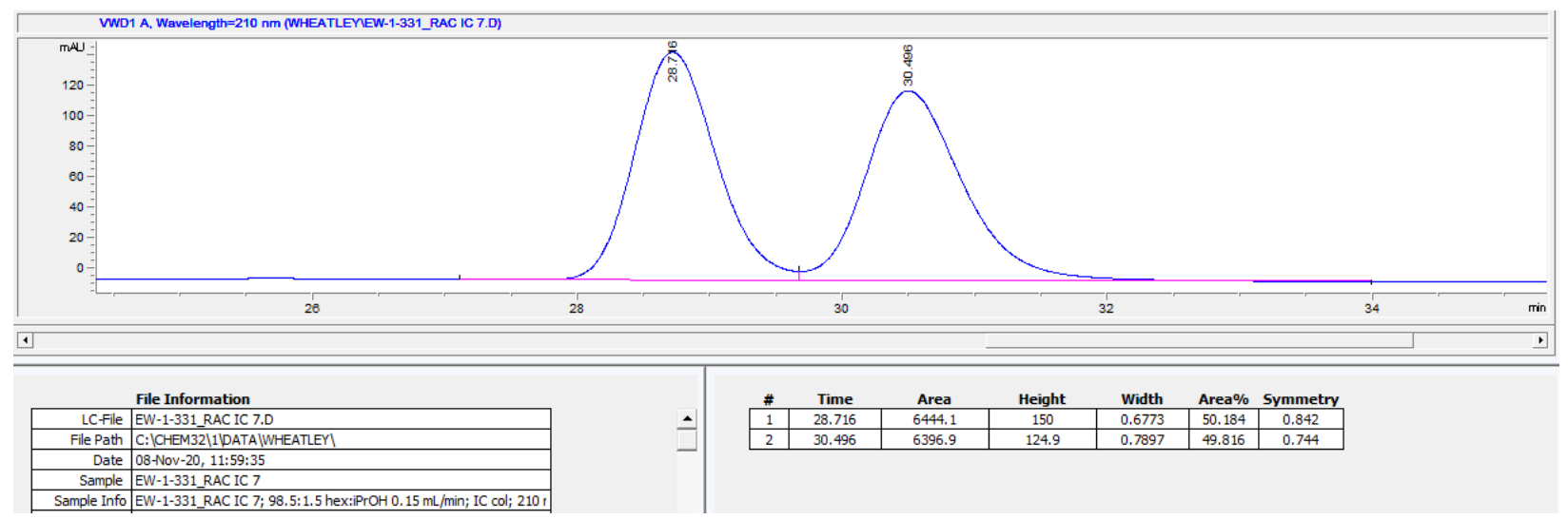

Enantioenriched material:

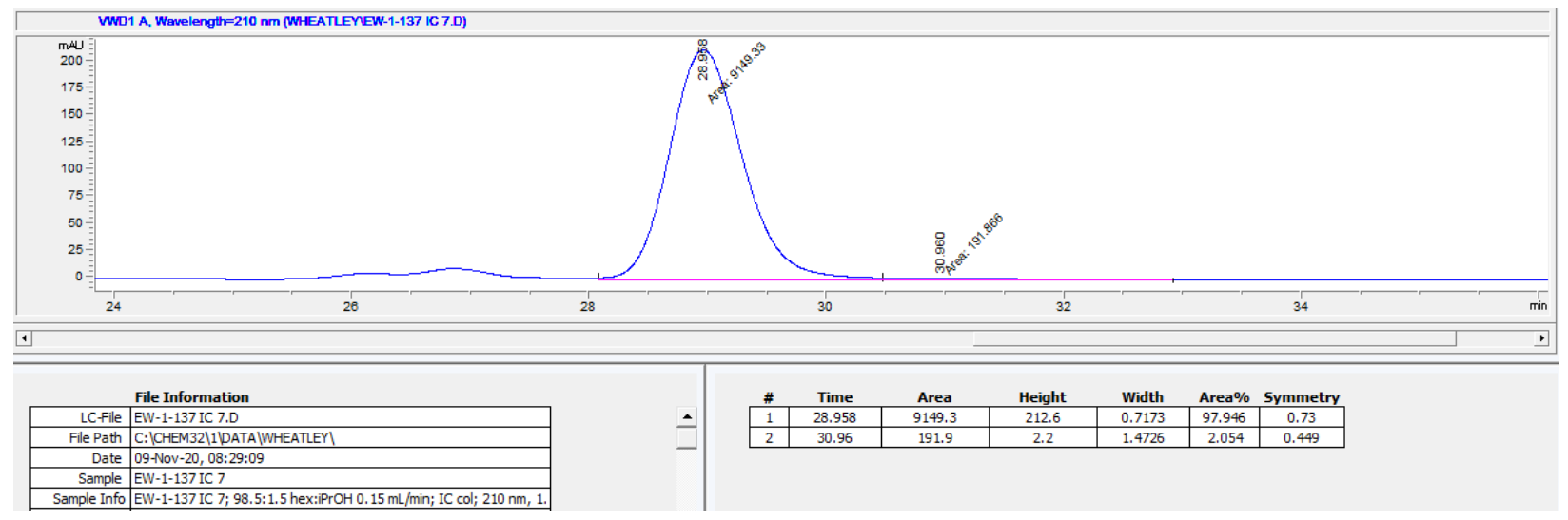

${ }^{1} \mathrm{H}$ NMR $\left(600 \mathrm{MHz}, \mathrm{CDCl}_{3}\right) \delta 7.01-6.97(\mathrm{~d}, 8.5 \mathrm{~Hz}, 2 \mathrm{H}), 6.78-6.74(\mathrm{~d}, 8.5 \mathrm{~Hz}, 2 \mathrm{H}), 6.61-6.55(\mathrm{~d}$, $18.4 \mathrm{~Hz}, 1 \mathrm{H}), 5.27-5.20(\mathrm{~d}, 18.4 \mathrm{~Hz}, 1 \mathrm{H}), 3.78(\mathrm{~s}, 3 \mathrm{H}), 2.55(\mathrm{~s}, 2 \mathrm{H}), 1.36-1.31(\mathrm{~m}, 1 \mathrm{H}), 1.28(\mathrm{~s}, 12 \mathrm{H})$, $1.26-1.21(\mathrm{~m}, 3 \mathrm{H}), 1.20-1.16(\mathrm{~m}, 2 \mathrm{H}), 0.90(\mathrm{~s}, 3 \mathrm{H}), 0.88-0.84(\mathrm{t}, 7.2 \mathrm{~Hz}, 1 \mathrm{H}) .{ }^{13} \mathrm{C}$ NMR $(151 \mathrm{MHz}$, $\left.\mathrm{CDCl}_{3}\right) \delta 162.5,157.9,131.7,130.7,113.0,83.1,55.3,46.6,42.4,39.7,26.7,25.0,24.8,23.6,22.1,14.3$. HRMS (m/z) calc. for $\left[\mathrm{M}+\mathrm{H}^{+}\right] \mathrm{C}_{22} \mathrm{H}_{36} \mathrm{BO}_{3}{ }^{+}: 359.2752$ found: 359.2752. IR (cm $\left.{ }^{-1}\right) 2958$ (s), 2930 (s), 1633 (s), 1512 (s), 1351 (s), 1248 (s), 1178 (s), 851 (w), 738 (w). [ $\alpha]_{\mathrm{D}^{23}}=-14.6$ (c = 0.82, $\mathrm{CH}_{2} \mathrm{Cl}_{2}, 1=$ $1 \mathrm{dm})$.

4-((2S,3S)-3-butyl-3-methyl-5-oxotetrahydrofuran-2-yl)benzonitrile (15)

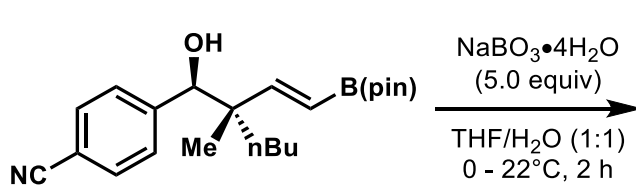

(3f)

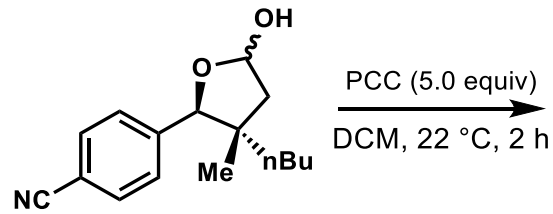

(S5)

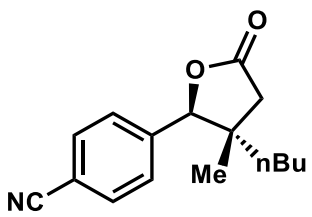

(15)

To an $8 \mathrm{~mL}$ vial charged with magnetic stirrer bar, boronic ester (3f) (17.4 mg, $0.0471 \mathrm{mmol})$ was weighed. THF $(200 \mu \mathrm{L})$ and deionized $\mathrm{H}_{2} \mathrm{O}(200 \mu \mathrm{L})$ were added. The solution was cooled to 
$0{ }^{\circ} \mathrm{C}$ and sodium perborate tetrahydrate $\left(\mathrm{NaBO}_{3} \bullet 4 \mathrm{H}_{2} \mathrm{O}\right)(36.2 \mathrm{mg}, 0.236 \mathrm{mmol}, 5.0$ equiv) was added at once as a solid. The reaction covered with a loose-fitting cap and allowed to stir $30 \mathrm{~min}$ at $0{ }^{\circ} \mathrm{C}$. The reaction was then warmed to room temperature and allowed to stir for $2 \mathrm{~h}$. Upon completion, the reaction was quenched with saturated aqueous sodium thiosulfate $(1 \mathrm{~mL})$ and the aqueous layer extracted with EtOAc $(3 \times 1 \mathrm{~mL})$. The organic layers were combined, dried over $\mathrm{MgSO}_{4}$, filtered and concentrated by rotary evaporation providing crude hemiacetal (S5) which was used without further purification.

An $8 \mathrm{~mL}$ vial with a stir bar was charged with crude hemiacetal (S5) and dry DCM (500 $\mu \mathrm{L})$. Silica gel (100 mg) was added, followed by pyridinium chlorochromate (PCC) (37.0 mg, 0.170 mmol, 5.0 equiv) which was added in one portion. The mixture was stirred vigorously for 2 hours, after which the suspension was filtered through Celite and washed with DCM. The filtrate was concentrated by rotary evaporation. The crude material was purified by silica gel column chromatography (Hexanes/Ethyl Acetate 25:1, 20:1, 15:1, 10:1) to give the product (8.5 mg, 95\% yield, >20:1 dr) as a colorless oil.

${ }^{1} \mathrm{H}$ NMR $\left(600 \mathrm{MHz}, \mathrm{CDCl}_{3}\right) \delta 7.75-7.67(\mathrm{~d}, 8.0 \mathrm{~Hz}, 2 \mathrm{H}), 7.45-7.38(\mathrm{~d}, 8.0 \mathrm{~Hz}, 2 \mathrm{H}), 5.24(\mathrm{~s}, 1 \mathrm{H})$, $2.69-2.61(\mathrm{~d}, 16.9 \mathrm{~Hz}, 1 \mathrm{H}), 2.49-2.41(\mathrm{~d}, 16.9 \mathrm{~Hz}, 1 \mathrm{H}), 1.61-1.55(\mathrm{~m}, 2 \mathrm{H}), 1.43-1.36(\mathrm{~m}, 2 \mathrm{H})$, $1.33-1.25(\mathrm{~m}, 2 \mathrm{H}), 1.00-0.94(\mathrm{t}, 6.9 \mathrm{~Hz}, 3 \mathrm{H}), 0.70(\mathrm{~s}, 3 \mathrm{H}) .{ }^{13} \mathrm{C}$ NMR (151 MHz, CDCl $) \delta 175.5$, $140.9,132.3,126.8,118.5,112.4,87.8,44.9,42.2,38.5,27.1,23.3,20.5,14.1$. HRMS (m/z) calc. for [M$\left.\mathrm{H}^{+}\right] \mathrm{C}_{16} \mathrm{H}_{20} \mathrm{NO}_{2}{ }^{+}: 258.1489$ found: 258.1490. IR $\left(\mathrm{cm}^{-1}\right) 2959(\mathrm{~m}), 2929(\mathrm{~m}), 2858(\mathrm{w}), 2360(\mathrm{w}), 2229$ (m), $1787(\mathrm{~s}), 1458(\mathrm{w}), 1197(\mathrm{~m}), 1029(\mathrm{~m}), 842(\mathrm{w}) .[\alpha]_{\mathrm{D}^{23}}=+2.5\left(\mathrm{c}=0.43, \mathrm{CH}_{2} \mathrm{Cl}_{2}, \mathrm{l}=1 \mathrm{dm}\right)$.

(2S,3S)-3-butyl-3-methyl-2-(3-nitrophenyl)tetrahydrofuran (16)

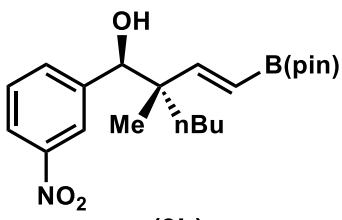

$(3 \mathrm{~h})$

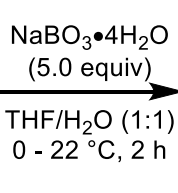

$0-22^{\circ} \mathrm{C}, 2 \mathrm{~h}$

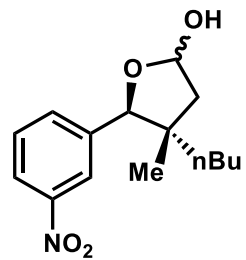

(S6)
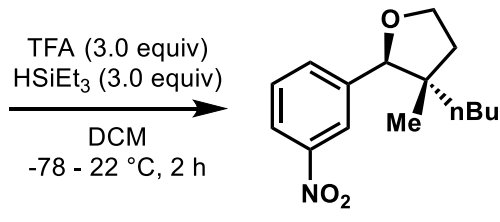

(16)

Under ambient conditions, substrate $3 \mathrm{~h}(17.0 \mathrm{mg}, 0.044 \mathrm{mmol}, 1.0$ equiv) was weighed into an $8 \mathrm{~mL}$ vial charged with magnetic stir bar. THF $(200 \mu \mathrm{L})$ was added followed by water $(200 \mu \mathrm{L})$ and the reaction mixture was cooled to $0{ }^{\circ} \mathrm{C}$ before adding $\mathrm{NaBO}_{3} \bullet 4 \mathrm{H}_{2} \mathrm{O}(33.6 \mathrm{mg}, 0.218 \mathrm{mmol})$. A cap was placed lightly on top and the reaction was allowed to stir for 10 minutes at $0{ }^{\circ} \mathrm{C}$ and then warmed to room temperature and stirred for 2 hours. Upon completion, the reaction mixture was cooled to $0{ }^{\circ} \mathrm{C}$ and quenched with $1 \mathrm{~mL}$ sat. $\mathrm{NaS}_{2} \mathrm{O}_{3}$ (aq). The organic layer was extracted with EtOAc $(3 \times 1 \mathrm{~mL})$, dried with $\mathrm{MgSO}_{4}$, filtered and concentrated by rotary evaporation. 
Based on a literature procedure ${ }^{6}$, an $8 \mathrm{~mL}$ vial was charged with the crude hemiacetal (S6) and dry DCM $(500 \mu \mathrm{L})$. The mixture was cooled to $-78^{\circ} \mathrm{C}$ and TFA (14.9 $\mathrm{mg}, 0.131 \mathrm{mmol}, 3.0$ equiv) was added followed by $\mathrm{Et}_{3} \mathrm{SiH}(15.2 \mathrm{mg}, 0.131 \mathrm{mmol}, 3.0$ equiv). After 10 minutes, the solution was allowed to warm to room temperature and stirred for 2 hours. The reaction was quenched was saturated $\mathrm{NaHCO}_{3}(1 \mathrm{~mL})$ and the aqueous phase extracted with $\mathrm{Et}_{2} \mathrm{O}(3 \times 1 \mathrm{~mL})$. The organic layers were combined, dried over $\mathrm{MgSO}_{4}$, filtered, and concentrated by rotary evaporation. The crude material was purified by silica gel column chromatography (Hexanes/Ethyl Acetate 25:1, 20:1, 15:1, 10:1) to give tetrahydrofuran (16) (5.6 mg, 50\% yield, >20:1 dr) as a colorless oil.

${ }^{1} \mathrm{H}$ NMR $\left(600 \mathrm{MHz}, \mathrm{CDCl}_{3}\right) \delta 8.21(\mathrm{~s}, 1 \mathrm{H}), 8.17$ - $8.12(\mathrm{~d}, 8.2 \mathrm{~Hz}, 1 \mathrm{H}), 7.70-7.63(\mathrm{~d}, 7.6 \mathrm{~Hz}, 1 \mathrm{H})$, $7.57-7.48(\mathrm{t}, 7.9 \mathrm{~Hz}, 1 \mathrm{H}), 5.81-5.73(\mathrm{~m}, 1 \mathrm{H}), 4.87(\mathrm{~s}, 1 \mathrm{H}), 2.20-2.11(\mathrm{dd}, 13.4,6.0 \mathrm{~Hz}, 1 \mathrm{H}), 1.99-$ $1.93(\mathrm{dd}, 13.4,4.5 \mathrm{~Hz}, 1 \mathrm{H}), 1.54-1.50(\mathrm{dd}, 12.2,4.6 \mathrm{~Hz}, 1 \mathrm{H}), 1.42$ - $1.33(\mathrm{~m}, 4 \mathrm{H}), 1.30$ - $1.22(\mathrm{~m}$, 2H), $0.99-0.88(\mathrm{t}, 6.9 \mathrm{~Hz}, 3 \mathrm{H}), 0.58(\mathrm{~s}, 3 \mathrm{H}) .{ }^{13} \mathrm{C} \mathbf{N M R}\left(151 \mathrm{MHz}, \mathrm{CDCl}_{3}\right) \delta$ 148.2, 140.8, 132.8, 129.0, 122.6, 121.7, 99.6, 86.1, 46.7, 46.0, 38.6, 27.5, 23.6, 21.0, 14.2. HRMS (m/z) calc. for $\left[{\left.\mathrm{M}+\mathrm{H}^{+}\right]}^{+}\right.$ $\mathrm{C}_{15} \mathrm{H}_{22} \mathrm{NO}_{3}{ }^{+}: 264.1594$ found: 264.1592. IR (cm-1) $2930(\mathrm{w}), 1532(\mathrm{~s}), 1466(\mathrm{w}), 1349$ (s), $981(\mathrm{~s}) .[\alpha]_{\mathrm{D}^{23}}$ $=+31.5\left(\mathrm{c}=0.80, \mathrm{CH}_{2} \mathrm{Cl}_{2}, \mathrm{l}=1 \mathrm{dm}\right)$.

tert-butyl-3-((1S,2S)-1-hydroxy-2-methyl-2-((E)-2-(pyridin-3-yl)vinyl)hexyl)-1H-indole-1-carboxylate (17)

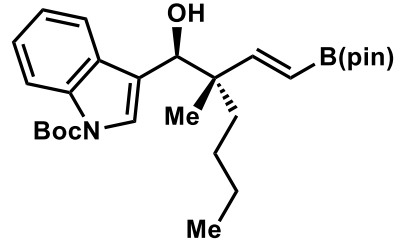

$(3 n)$

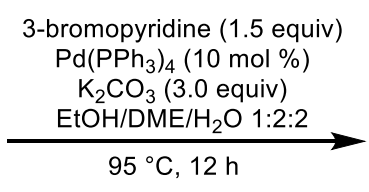

$95^{\circ} \mathrm{C}, 12 \mathrm{~h}$

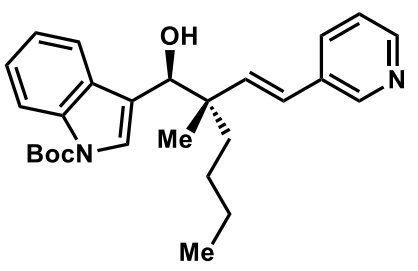

(17)

In a $\mathrm{N}_{2}$ filled glove box, vinyl boronic ester (3n) $(50.0 \mathrm{mg}, 0.10 \mathrm{mmol})$ was weighed into an 8 $\mathrm{mL}$ vial charged with magnetic stirrer bar. Into the same vial, $\mathrm{Pd}\left(\mathrm{PPh}_{3}\right)_{4}(12.0 \mathrm{mg}, 10 \mathrm{~mol} \%)$ and DME $(300 \mu \mathrm{L})$ were added. The vial was sealed with a septa cap and electrical tape before removing from the glove box. Ethanol $(150 \mu \mathrm{L})$, 3-bromopyridine $(25 \mathrm{mg}, 0.16 \mathrm{mmol})$ and sat. $\mathrm{K}_{2} \mathrm{CO}_{3}$ solution $(300 \mu \mathrm{L})$ were added sequentially. The reaction mixture was heated to $95{ }^{\circ} \mathrm{C}$ (mineral oil bath or aluminum reaction block) and allowed to stir for 12 hours. Upon completion, the reaction was cooled to room temperature and $1 \mathrm{~mL}$ saturated aqueous $\mathrm{NH}_{4} \mathrm{Cl}$ was added. The crude mixture was extracted with EtOAc $(3 \times 1 \mathrm{~mL})$, dried with $\mathrm{MgSO}_{4}$, filtered and concentrated by rotary evaporation. The crude material was purified by silica gel column chromatography (Hexanes/Ethyl Acetate 25:1, 20:1, 15:1, 10:1) to give the product (17) (19.7 mg, 44\% yield, 13:1 dr) as a colorless oil.

${ }^{1} \mathbf{H}$ NMR (600 MHz, CDCl $) \delta 8.55$ (s, 1H), 8.43 (s, 1H), 8.14 (s, 1H), 7.69 - $7.65(\mathrm{~m}, 1 \mathrm{H}), 7.61$ - 7.57 $(\mathrm{d}, 7.8 \mathrm{~Hz}, 1 \mathrm{H}), 7.51(\mathrm{~s}, 1 \mathrm{H}), 7.31-7.27(\mathrm{t}, 7.7 \mathrm{~Hz}, 1 \mathrm{H}), 7.24-7.20(\mathrm{dd}, 7.9,4.7 \mathrm{~Hz}, 1 \mathrm{H}), 7.20-7.16$ 
$(\mathrm{t}, 7.6 \mathrm{~Hz}, 1 \mathrm{H}), 6.45-6.39(\mathrm{~d}, 16.4 \mathrm{~Hz}, 1 \mathrm{H}), 6.57-6.30(\mathrm{~d}, 16.3 \mathrm{~Hz}, 1 \mathrm{H}), 4.89(\mathrm{~s}, 1 \mathrm{H}), 2.26(\mathrm{~s}, 1 \mathrm{H})$, $1.74-1.66(\mathrm{~m}, 1 \mathrm{H}), 1.60(\mathrm{~s}, 9 \mathrm{H}), 1.31-1.25(\mathrm{~m}, 4 \mathrm{H}), 1.22-1.17(\mathrm{~m}, 1 \mathrm{H}), 1.13(\mathrm{~s}, 3 \mathrm{H}), 0.91-0.83(\mathrm{t}$, $6.9 \mathrm{~Hz}, 3 \mathrm{H}) .{ }^{13} \mathrm{C}$ NMR $\left(151 \mathrm{MHz}, \mathrm{CDCl}_{3}\right) \delta 148.3,148.3,138.6,133.2,132.7,130.1,126.5,124.4,124.3$, 123.5, 122.6, 121.3, 120.4, 115.3, 83.9, 74.6, 46.1, 37.5, 28.2, 26.6, 25.0, 23.6, 18.6, 14.3. HRMS (m/z) calc. for $\left[\mathrm{M}+\mathrm{H}^{+}\right] \mathrm{C}_{27} \mathrm{H}_{35} \mathrm{~N}_{2} \mathrm{O}_{3}{ }^{+}$: 435.2642 found: 435.2639. IR (cm-1) 2931 (w), 1734 (s), 1452 (s), 1371 (s), $1272(\mathrm{w}), 1158(\mathrm{~s}), 1081(\mathrm{~s}) .[\alpha]_{\mathrm{D}^{23}}=-45.5\left(\mathrm{c}=0.99, \mathrm{CH}_{2} \mathrm{Cl}_{2}, \mathrm{l}=1 \mathrm{dm}\right)$.

tert-butyl-3-((1S,2S)-1-((tert-butyldimethylsilyl)oxy)-2-methyl-2-(2-oxoethyl)hexyl)-1H-indole-1carboxylate (S8)

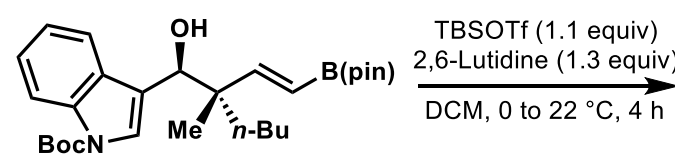

(3n)

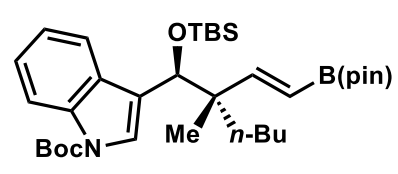

(S7)

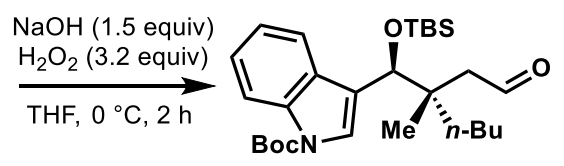

(S8)

Vinyl boronic ester (3n) was weighed into an $8 \mathrm{~mL}$ vial charged with magnetic stir bar, sealed with septa cap and placed under $\mathrm{N}_{2}$. DCM $(500 \mu \mathrm{L})$ was added and the solution was cooled to 0 ${ }^{\circ} \mathrm{C}$ (ice bath) before adding 2,6-lutidine (14 mg, $0.130 \mathrm{mmol}, 1.3$ equiv), followed by TBSOTf (29 $\mathrm{mg}, 0.110 \mathrm{mmol}, 1.1$ equiv). The reaction mixture was allowed to stir at $0{ }^{\circ} \mathrm{C}$ for 1 hour and then allowed to warm to room temperature and stir for an additional 3 hours. Upon completion the reaction was quenched with saturated aqueous $\mathrm{NaHCO}_{3}(1 \mathrm{~mL})$, extracted with EtOAc $(3 \times 1 \mathrm{~mL})$, dried over $\mathrm{MgSO}_{4}$, filtered and concentrated by rotary evaporation. The crude silyl ether (S7) product was used without further purification.

An $8 \mathrm{~mL}$ vial was charged with crude silyl ether (S7) and dissolved in THF (500 $\mu \mathrm{L})$ and cooled to $0{ }^{\circ} \mathrm{C}$ (ice bath) before addition of $\mathrm{NaOH}(3 \mathrm{M}, 49 \mu \mathrm{L})$ and $\mathrm{H}_{2} \mathrm{O}_{2}(30 \%, 32 \mu \mathrm{L})$. The reaction mixture was sealed with a septum lined cap that was pierced with a vent needle and allowed to warm to room temperature and stir for 3 hours. The reaction was quenched with a saturated solution of sodium thiosulphate $(1 \mathrm{~mL})$, extracted with $\mathrm{Et}_{2} \mathrm{O}(3 \times 1 \mathrm{~mL})$, dried over $\mathrm{MgSO}_{4}$, filtered and concentrated by rotary evaporation. The crude aldehyde (S8) product was used without further purification.

tert-butyl-3-((1S,2S,4R,5S,E)-2-butyl-1-((tert-butyldimethylsilyl)oxy)-5-ethyl-4-hydroxy-2,5dimethyl-7-(4,4,5,5-tetramethyl-1,3,2-dioxaborolan-2-yl)hept-6-en-1-yl)-1H-indole-1carboxylate (18)

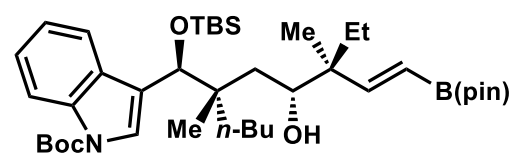

(18) 
Vinyl boronic ester (18) was prepared according to General Procedure III with crude aldehyde (S8) and diboron reagent (2b). Purified by silica gel column chromatography (Hexanes/Ethyl Acetate 25:1, 20:1, 15:1, 10:1) to give the product (18) (13.2 mg, $24 \%$ yield, 9:1 dr) as a colorless oil.

${ }^{1} \mathrm{H}$ NMR $\left(600 \mathrm{MHz}, \mathrm{CDCl}_{3}, 25^{\circ} \mathrm{C}\right.$ and $\left.55^{\circ} \mathrm{C}\right) \delta 8.18$ (br s, 1H), 7.90 (br s, 1H), 7.47 (br s, 1H), 7.32 - $7.27(\mathrm{~m}, 1 \mathrm{H}), 7.25-7.19(\mathrm{t}, 7.4 \mathrm{~Hz}, 1 \mathrm{H}), 6.67-6.56(\mathrm{~d}, 18.5 \mathrm{~Hz}, 1 \mathrm{H}), 5.49-5.40(\mathrm{~d}, 18.5 \mathrm{~Hz}, 1 \mathrm{H})$, $4.90(\mathrm{~s}, 1 \mathrm{H}), 3.61-3.54(\mathrm{~d}, 8.8 \mathrm{~Hz}, 1 \mathrm{H}), 1.68(\mathrm{~s}, 9 \mathrm{H}), 1.42-1.38(\mathrm{~m}, 1 \mathrm{H}), 1.36-1.29(\mathrm{~m}, 4 \mathrm{H}), 1.26(\mathrm{~s}$, $12 \mathrm{H}), 1.24-1.19(\mathrm{~m}, 4 \mathrm{H}), 1.01$ (br s, 2H), $0.95-0.91(\mathrm{t}, 7.0 \mathrm{~Hz}, 3 \mathrm{H}), 0.91-0.89(\mathrm{~m}, 3 \mathrm{H}), 0.88(\mathrm{~s}, 9 \mathrm{H})$, $0.83-0.78(\mathrm{t}, 7.5 \mathrm{~Hz}, 3 \mathrm{H}), 0.77-0.72(\mathrm{~m}, 3 \mathrm{H}), 0.04(\mathrm{~s}, 3 \mathrm{H}),-0.32$ (br m, 3H). ${ }^{13} \mathrm{C}$ NMR $(151 \mathrm{MHz}$, $\left.\mathrm{CDCl}_{3}, 55^{\circ} \mathrm{C}\right) \delta 162.7,160.4,149.9,125.7,124.1,122.5,121.6,115.3,109.0,104.5,83.6,83.1,83.0,82.6$, 73.3, 41.9, 36.9, 36.3, 31.7, 30.6, 29.9, 28.4, 26.1, 25.0, 25.0, 23.8, 18.4, 14.3, 8.8, -4.3, -5.0. HRMS (m/z)

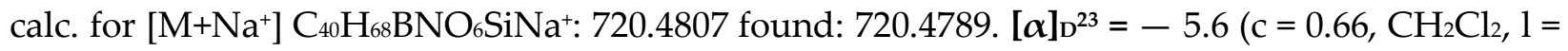
$1 \mathrm{dm}$ ). 


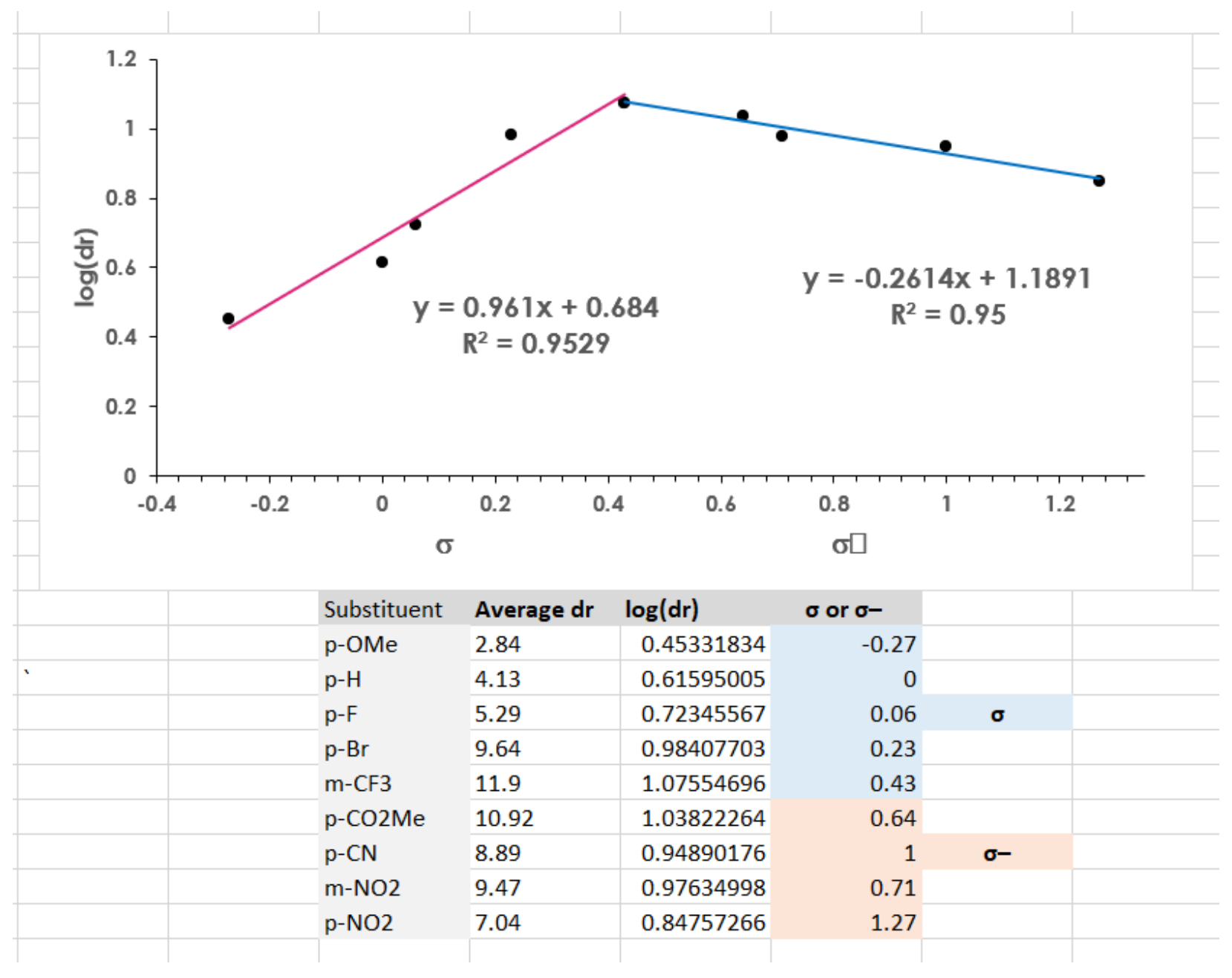




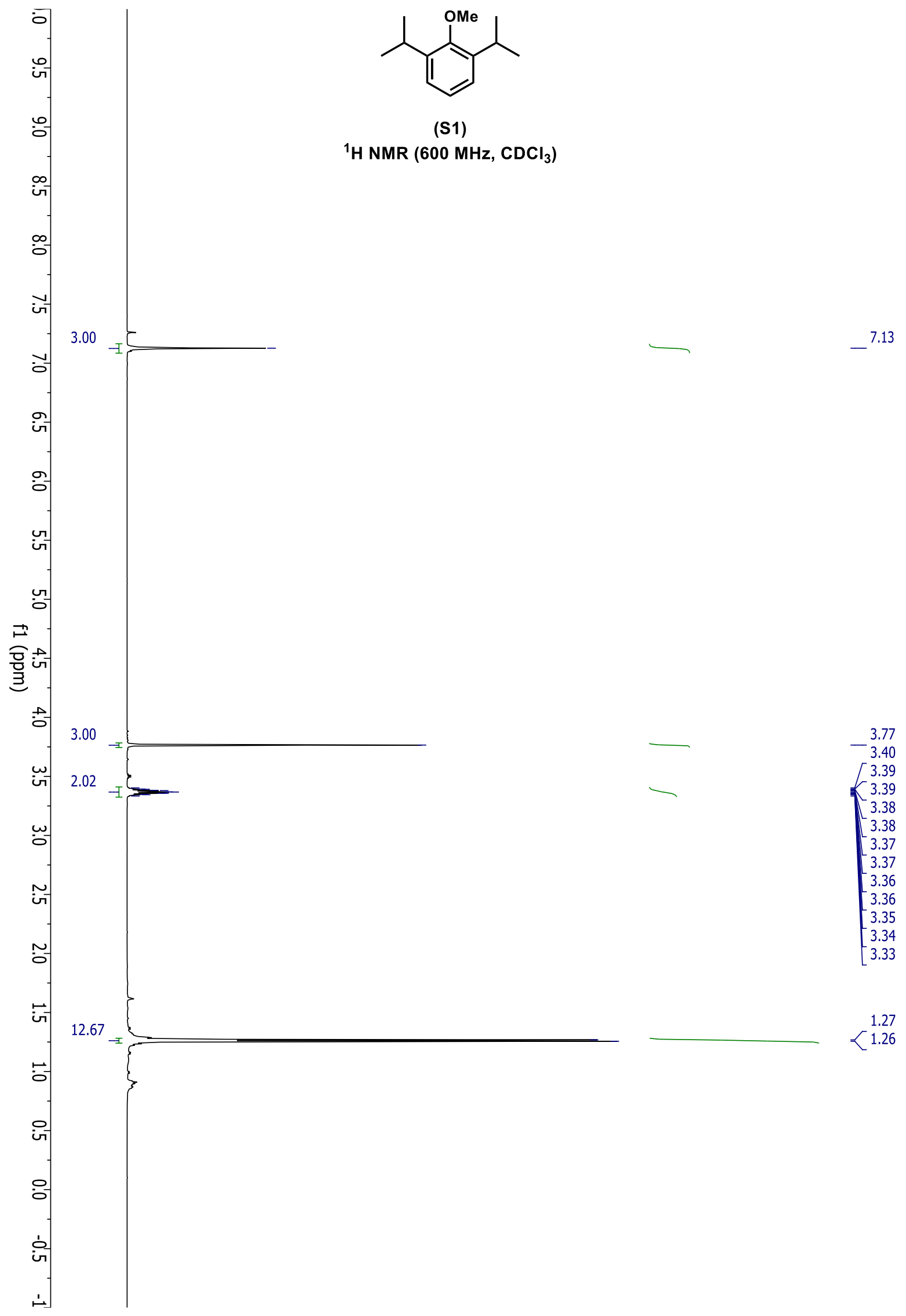




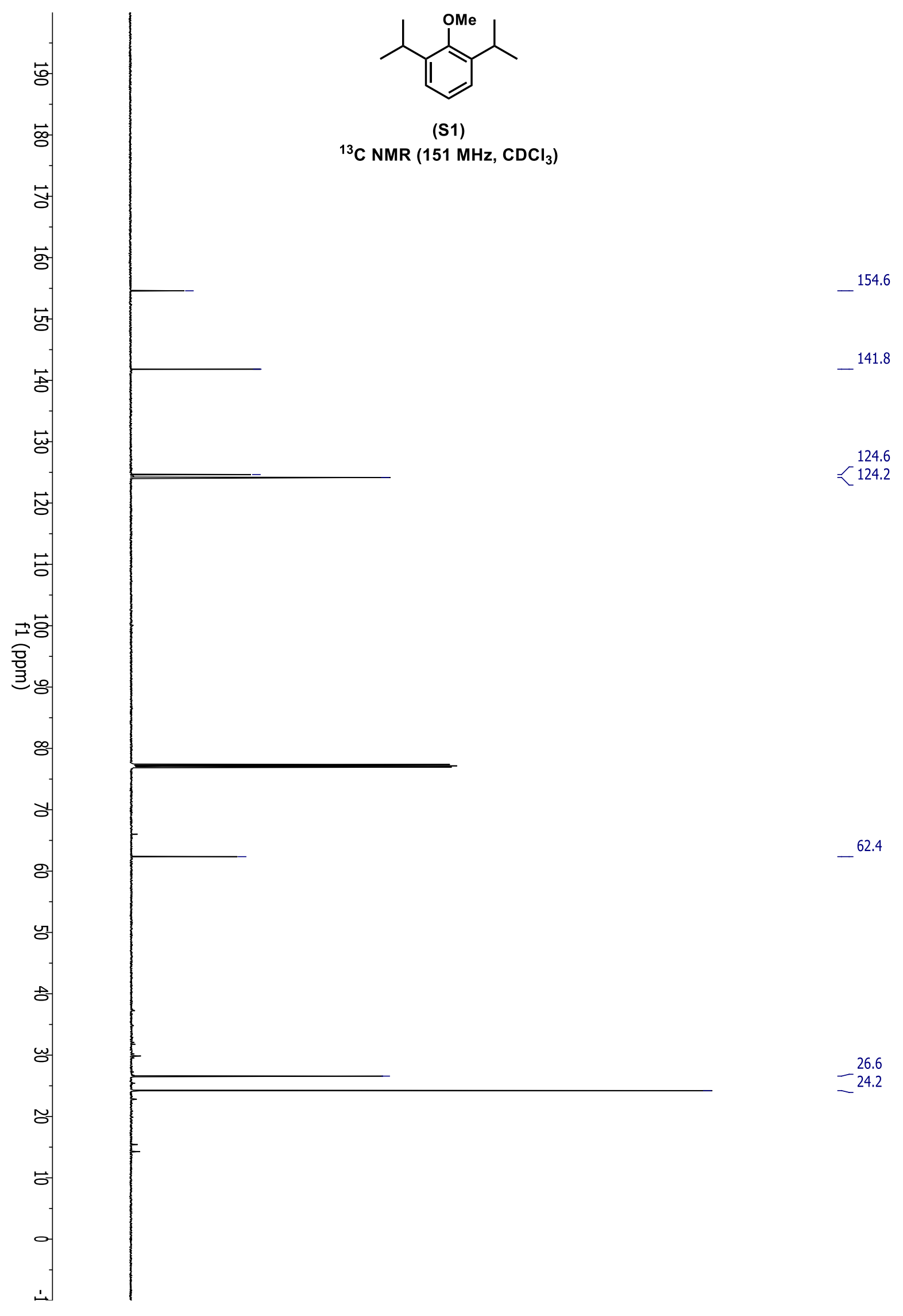




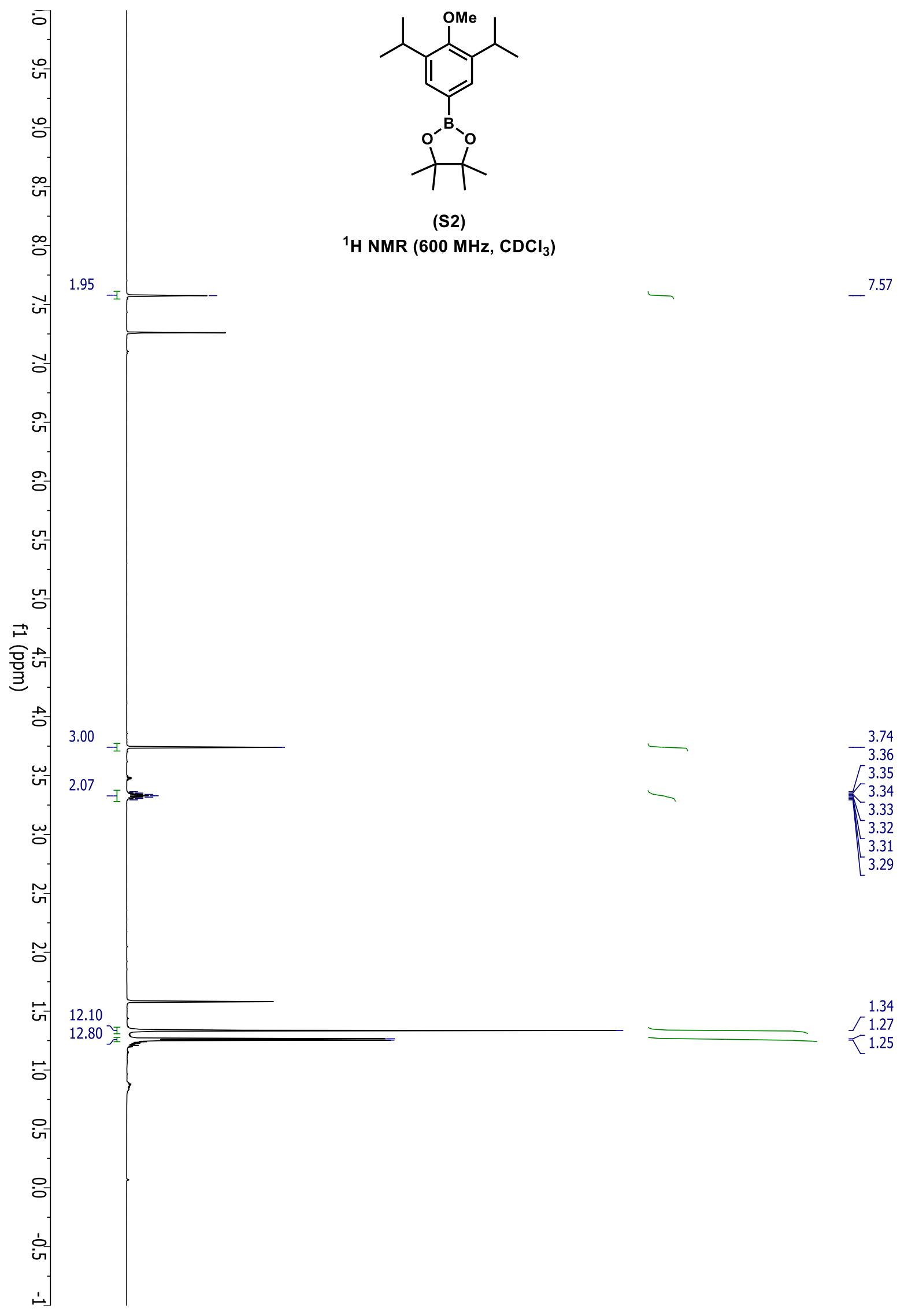




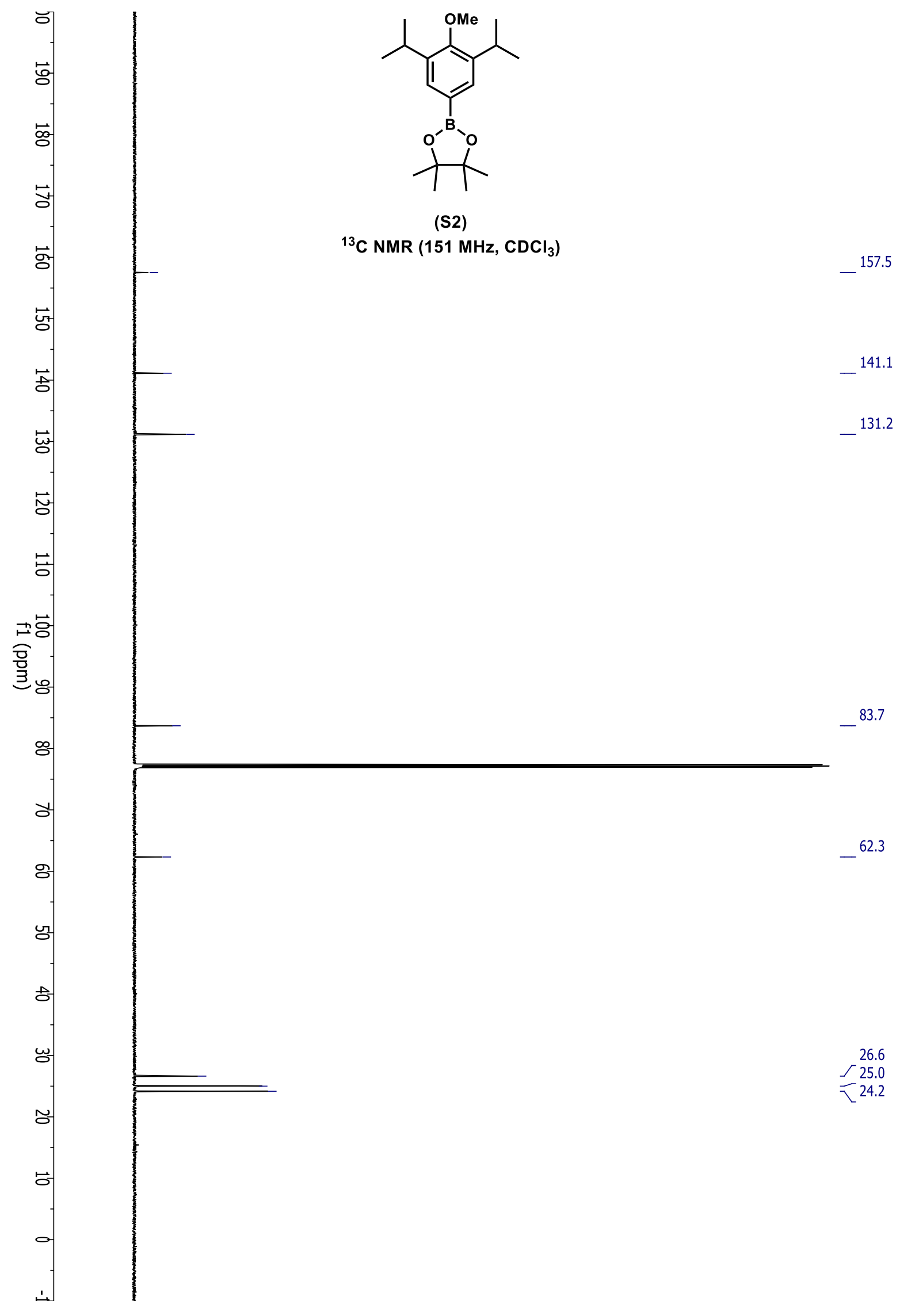




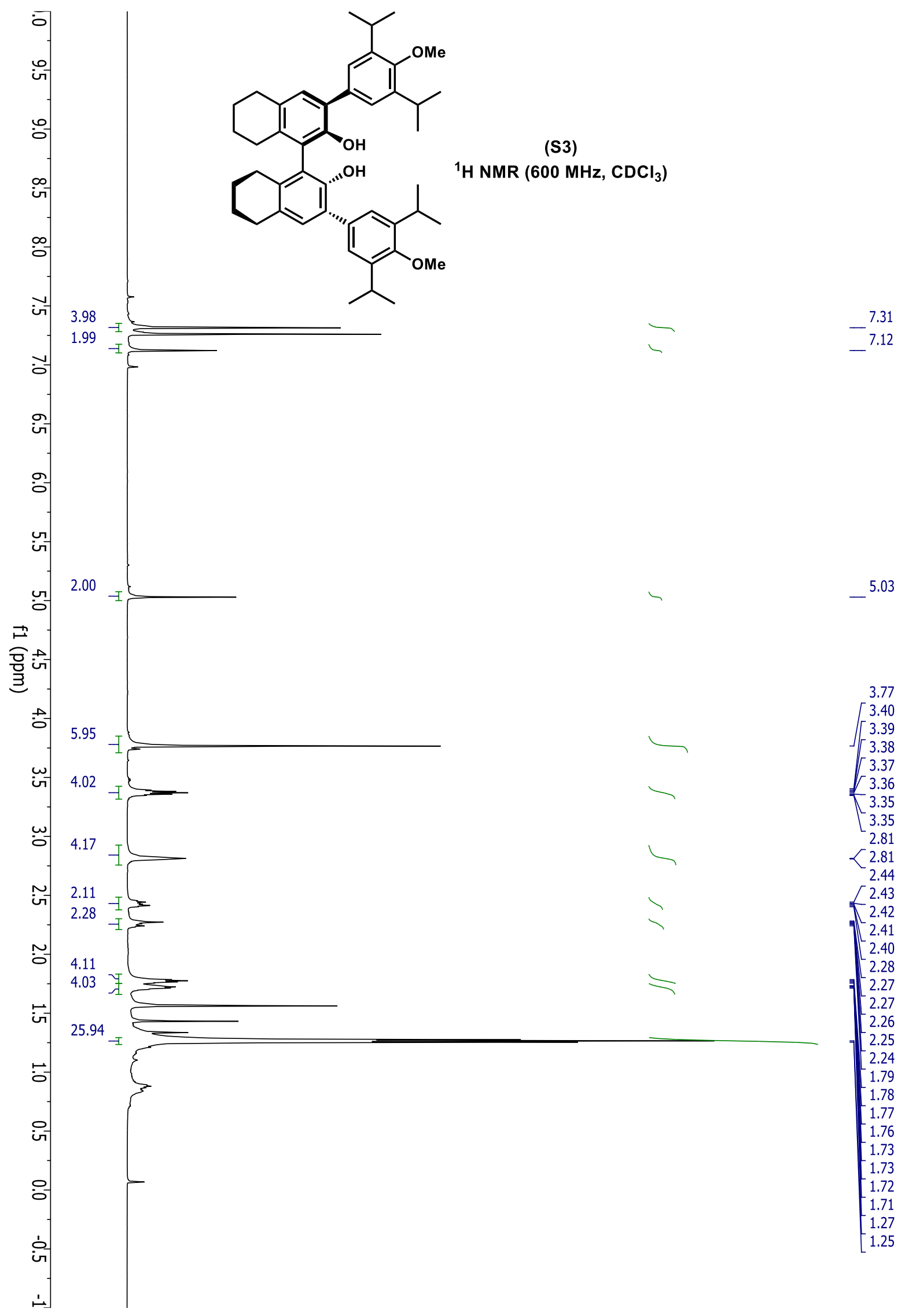




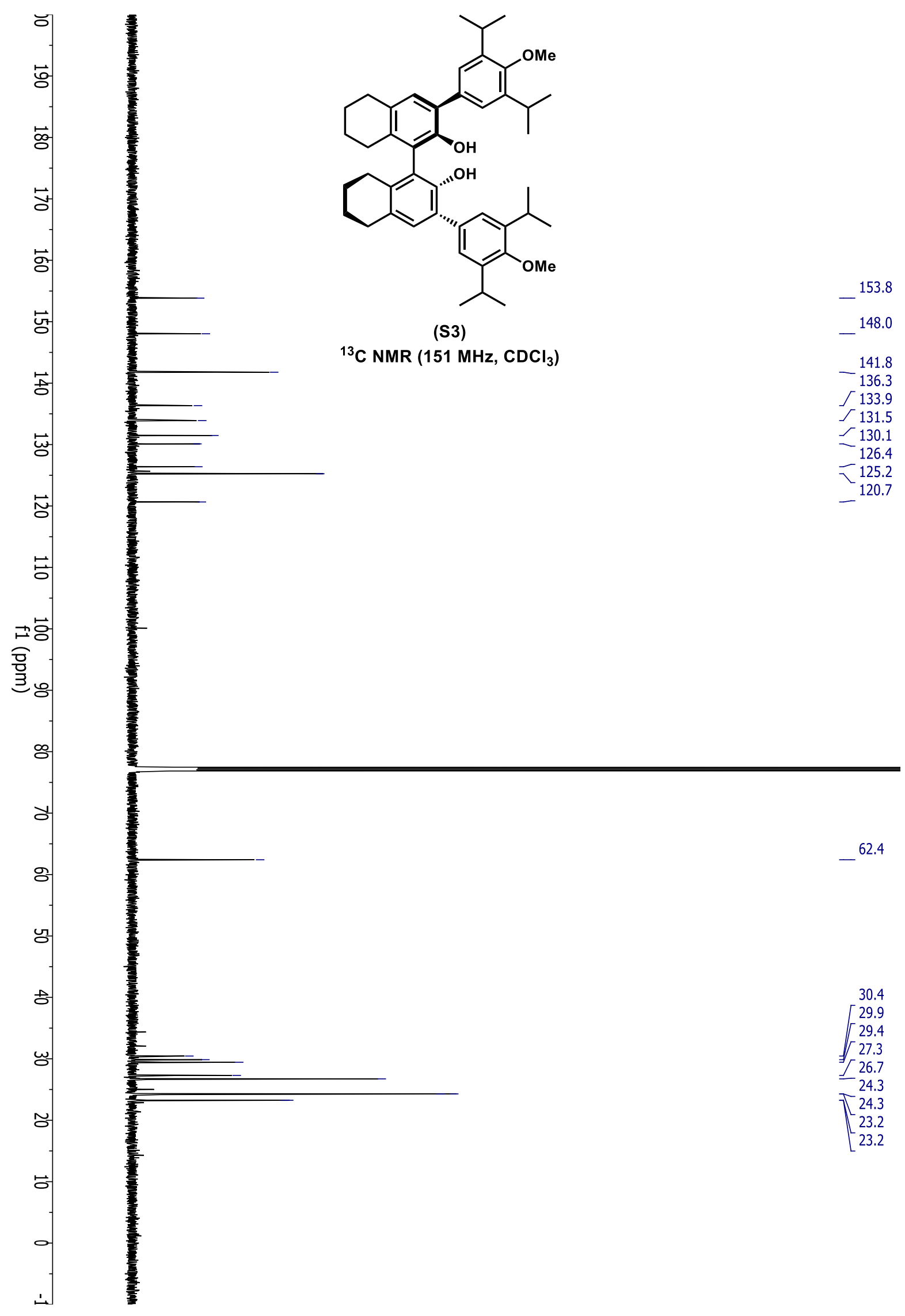




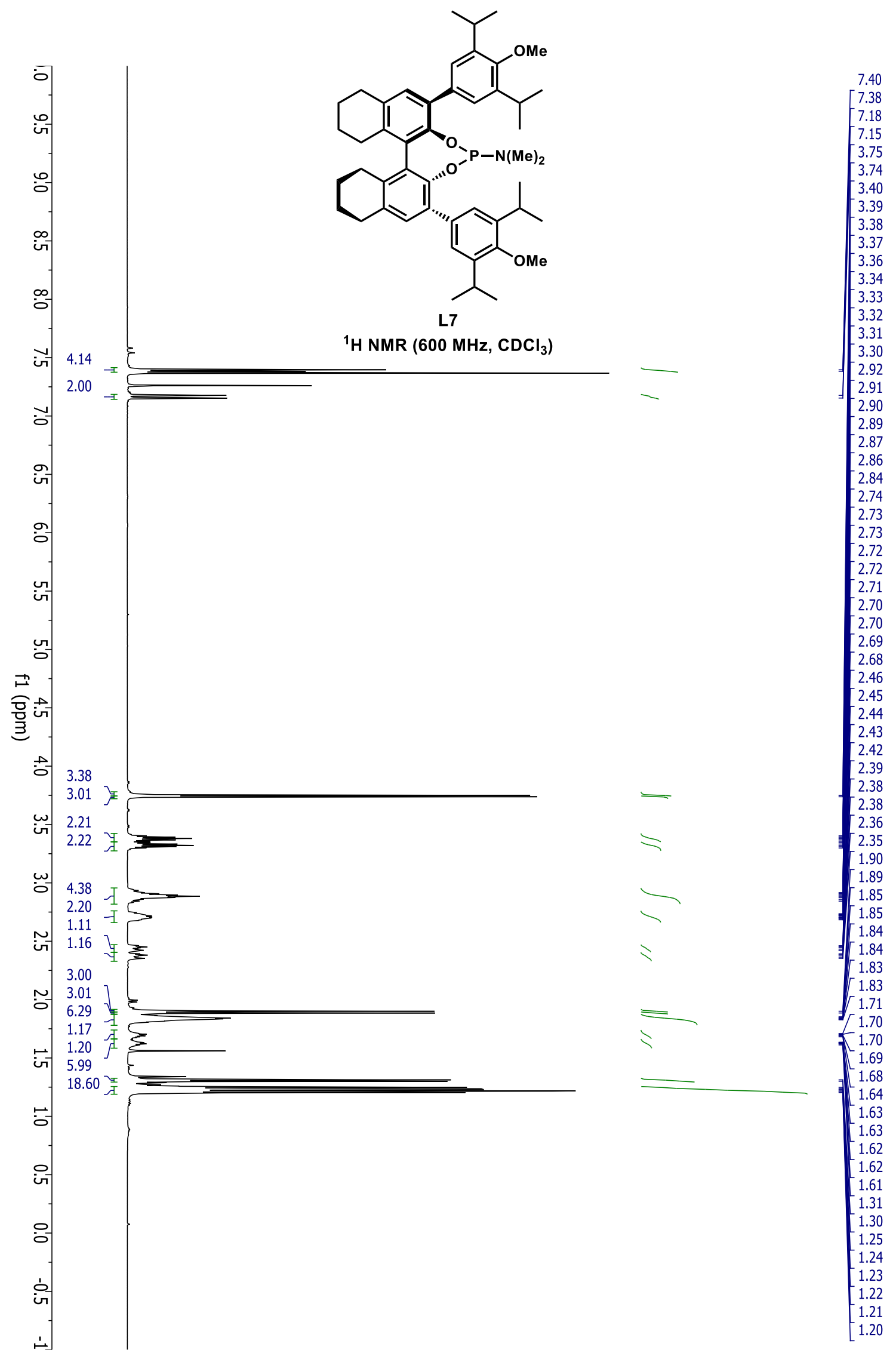




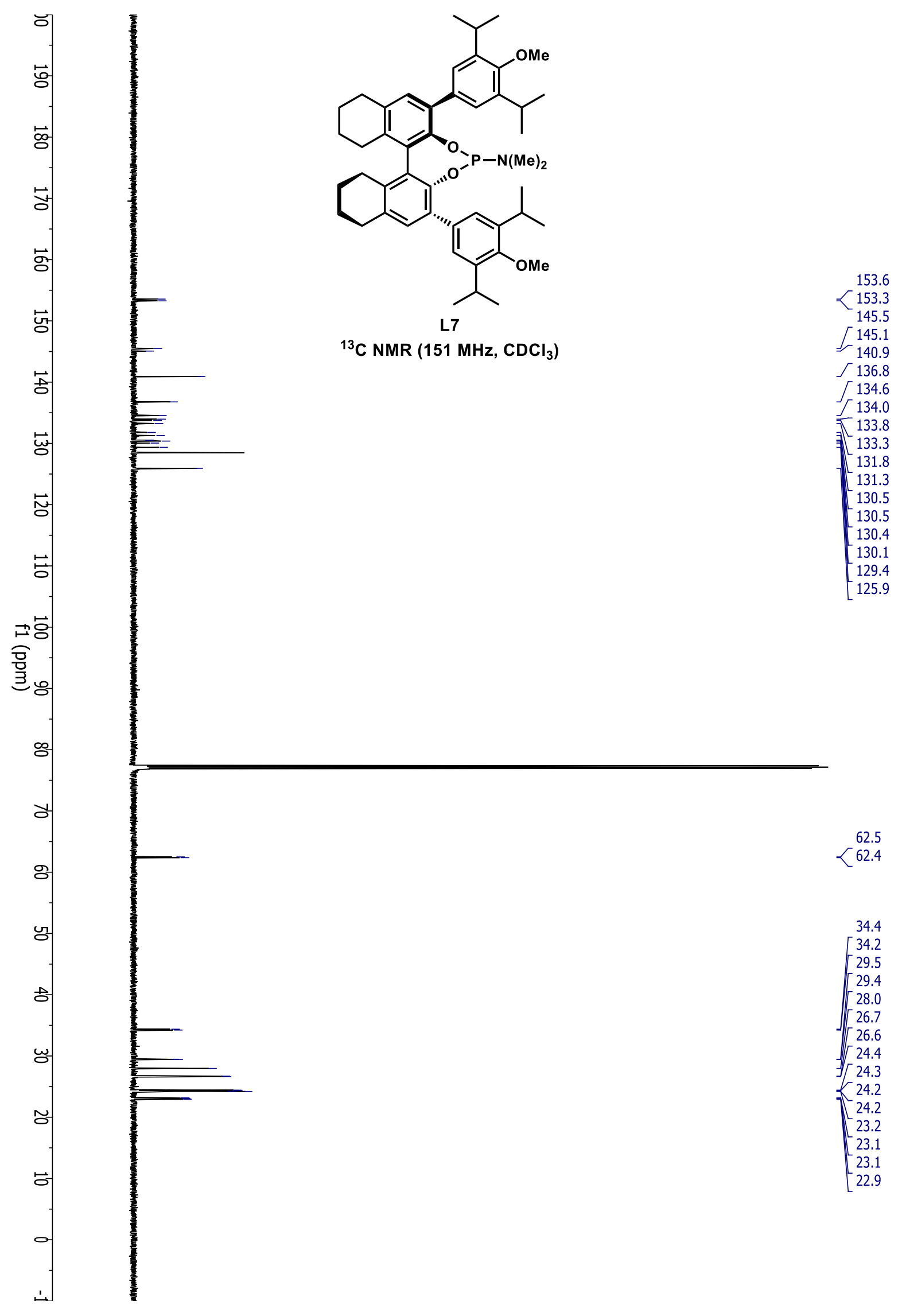




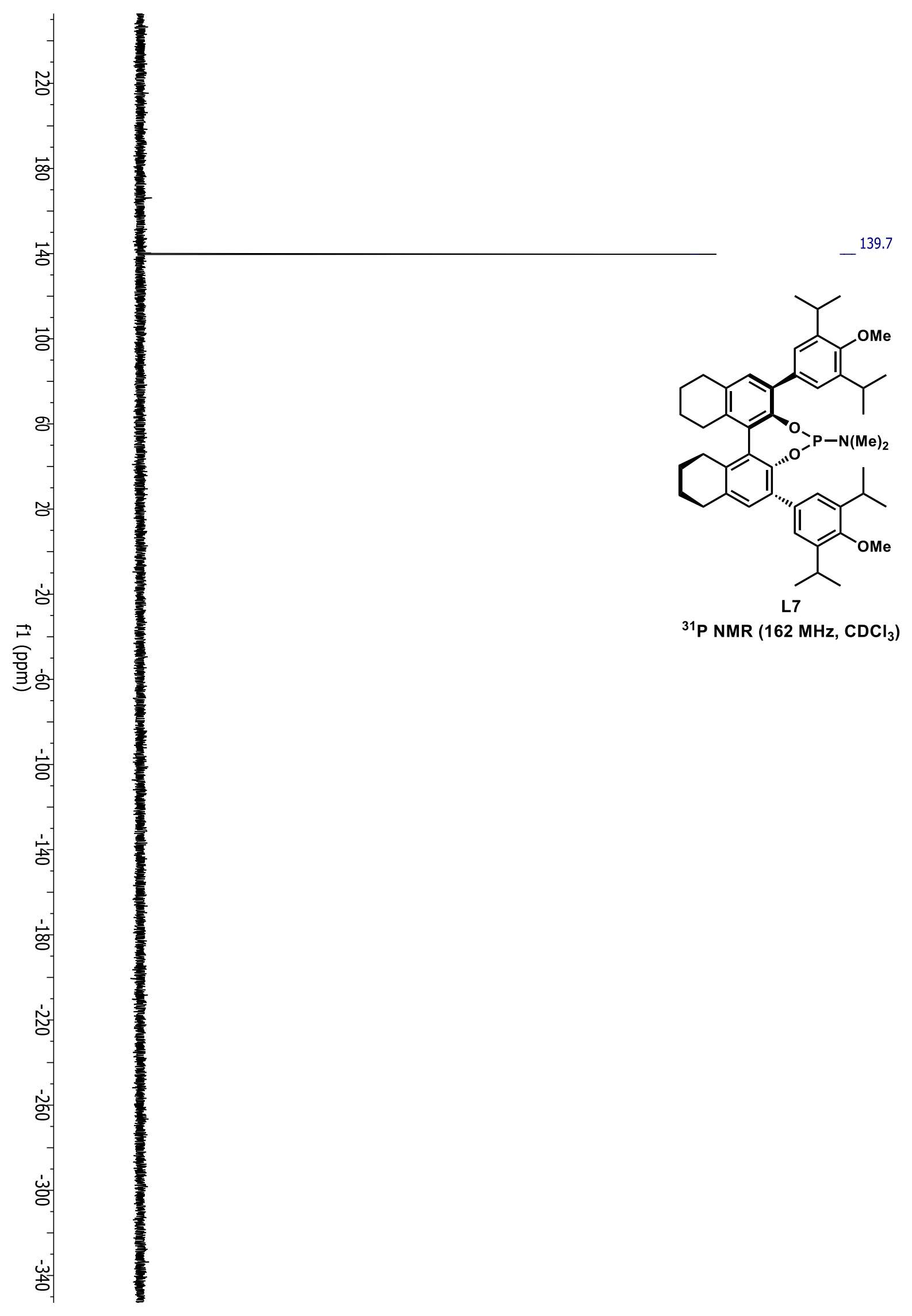




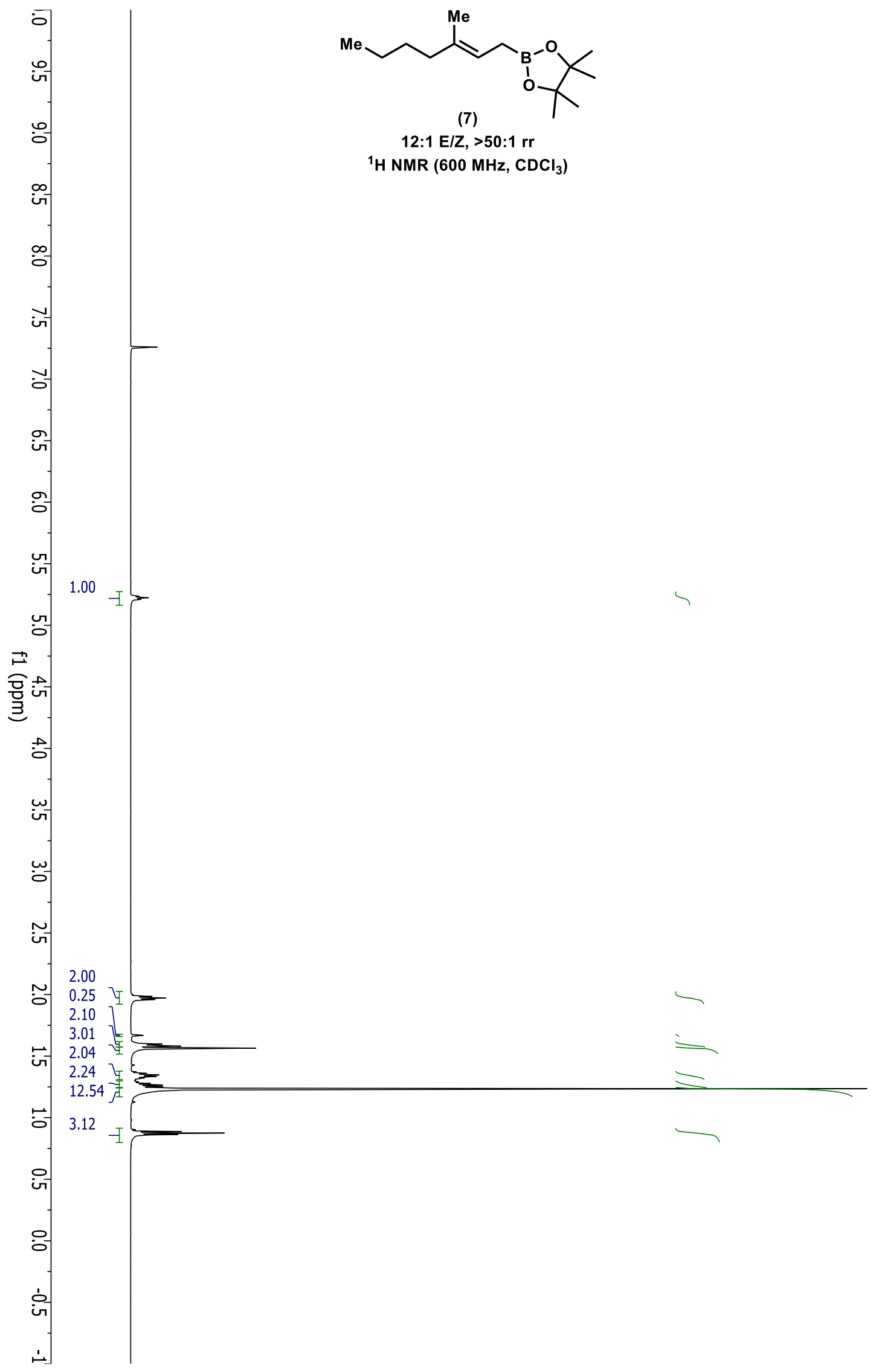




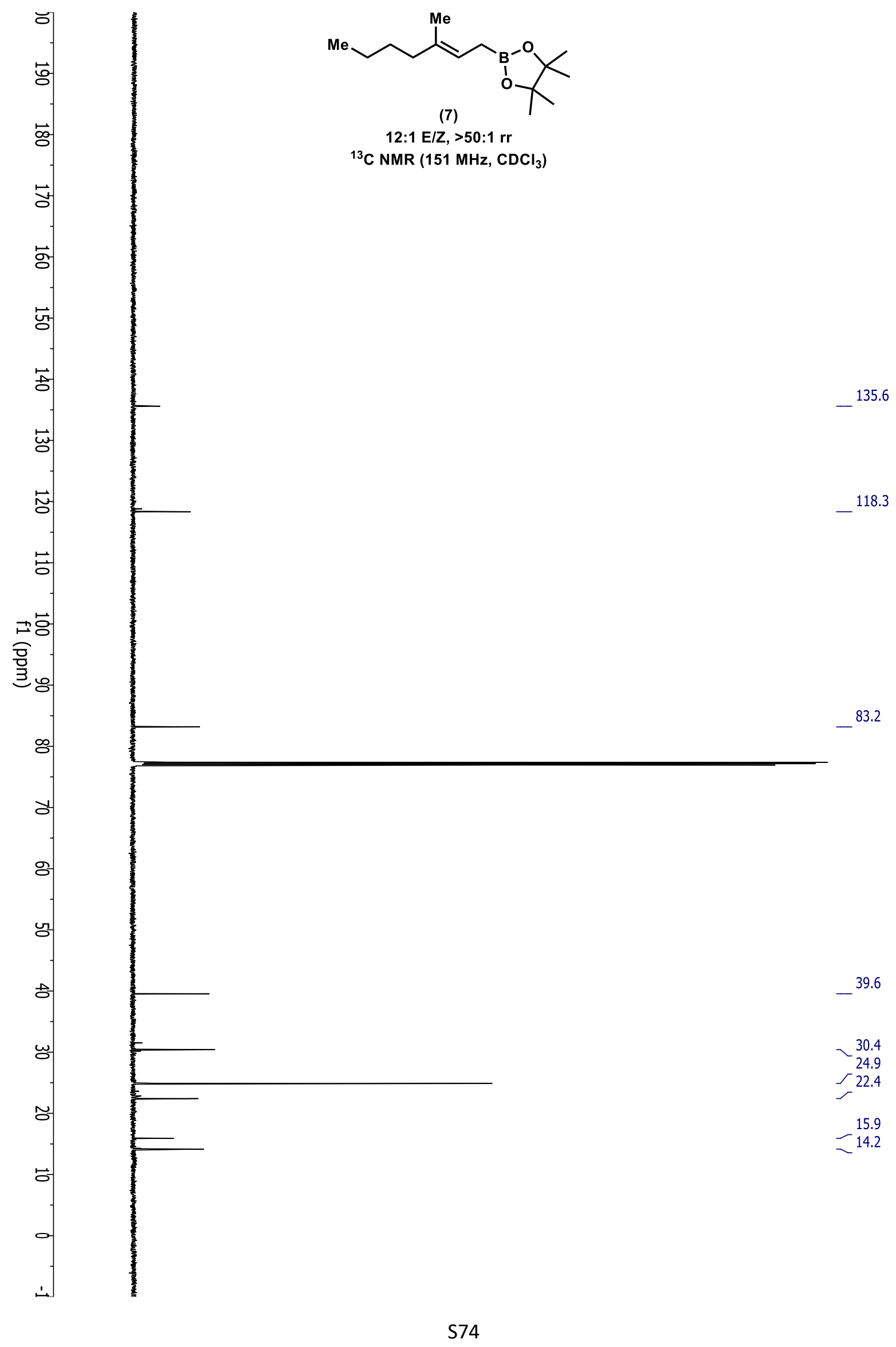



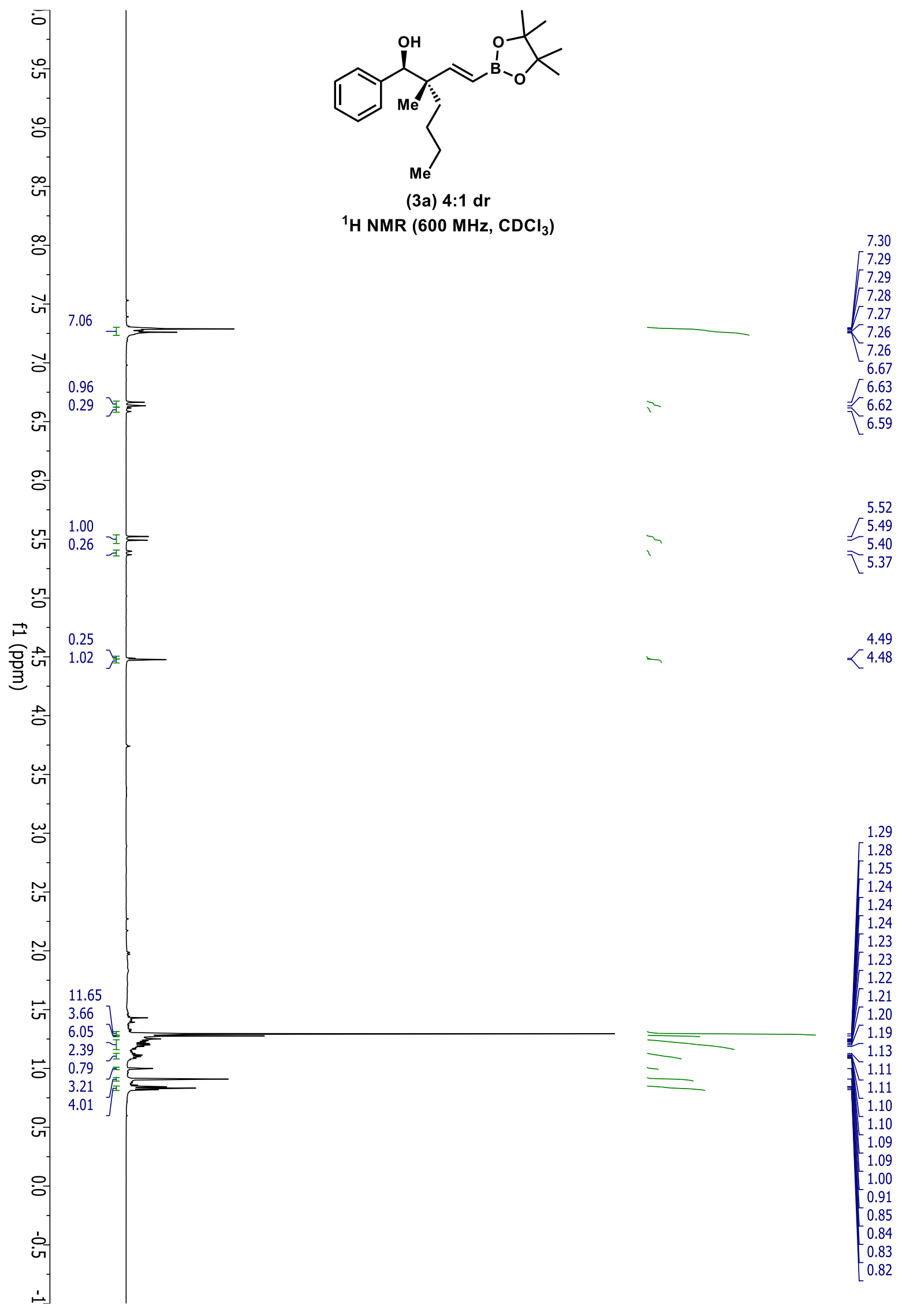


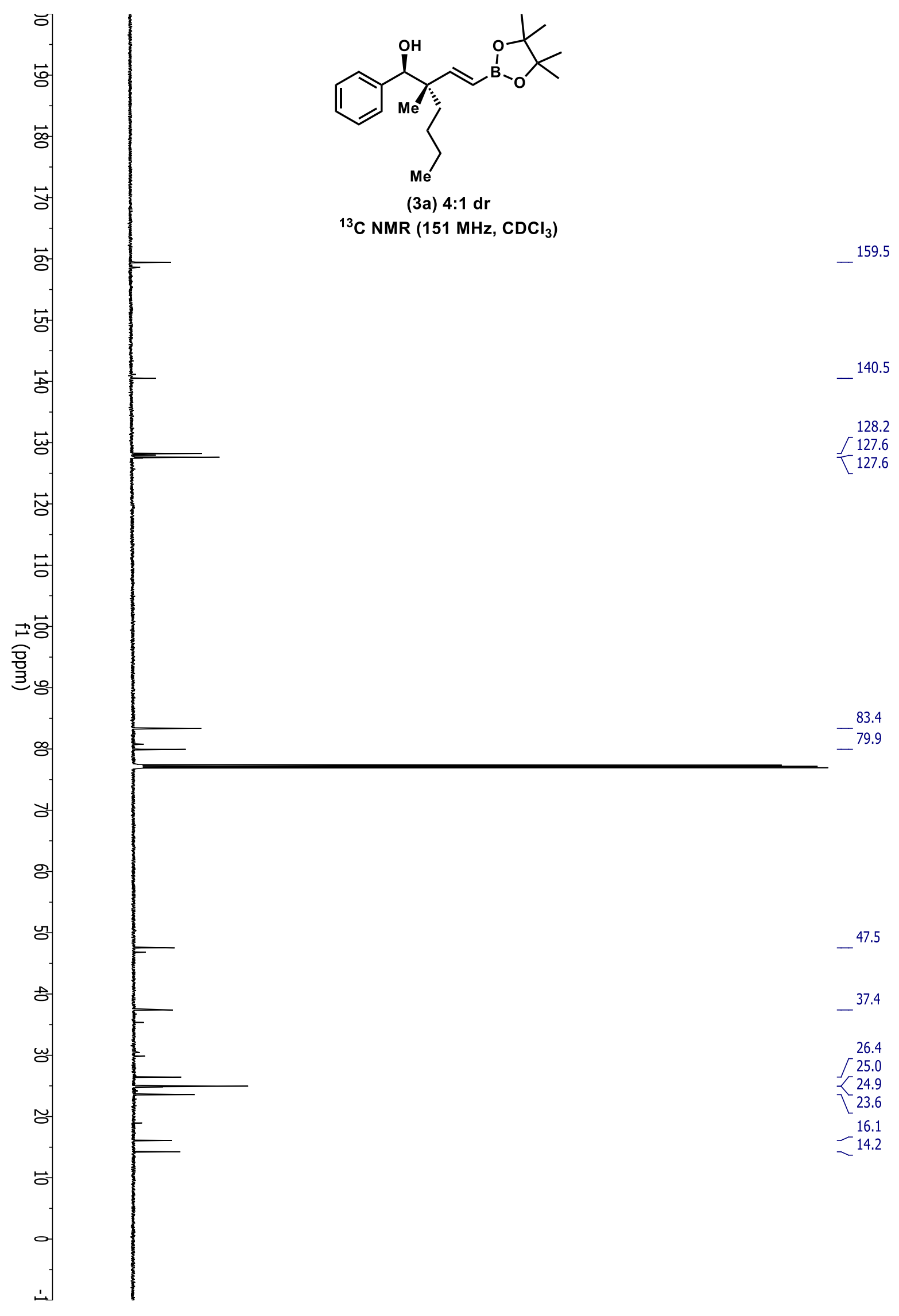




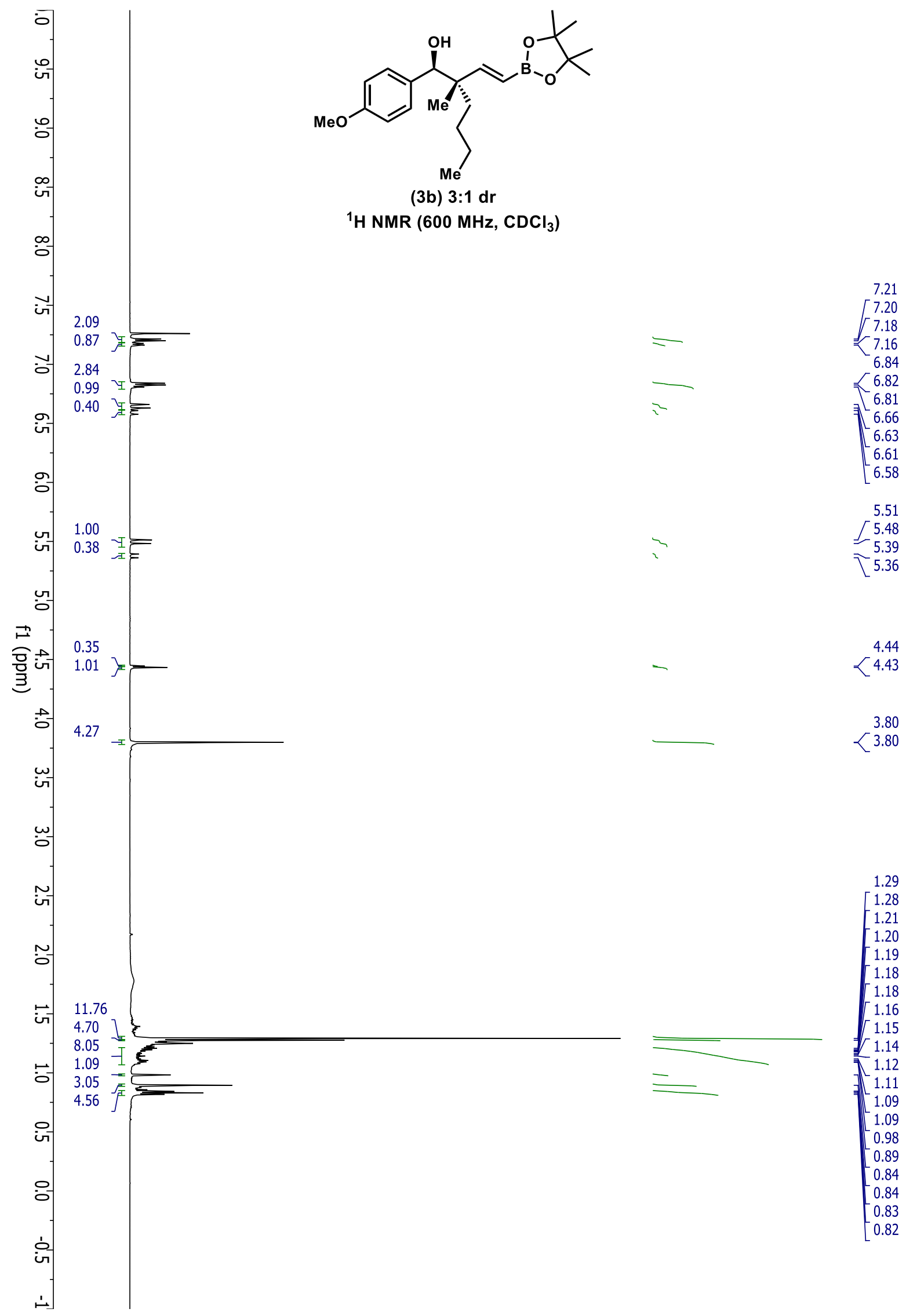




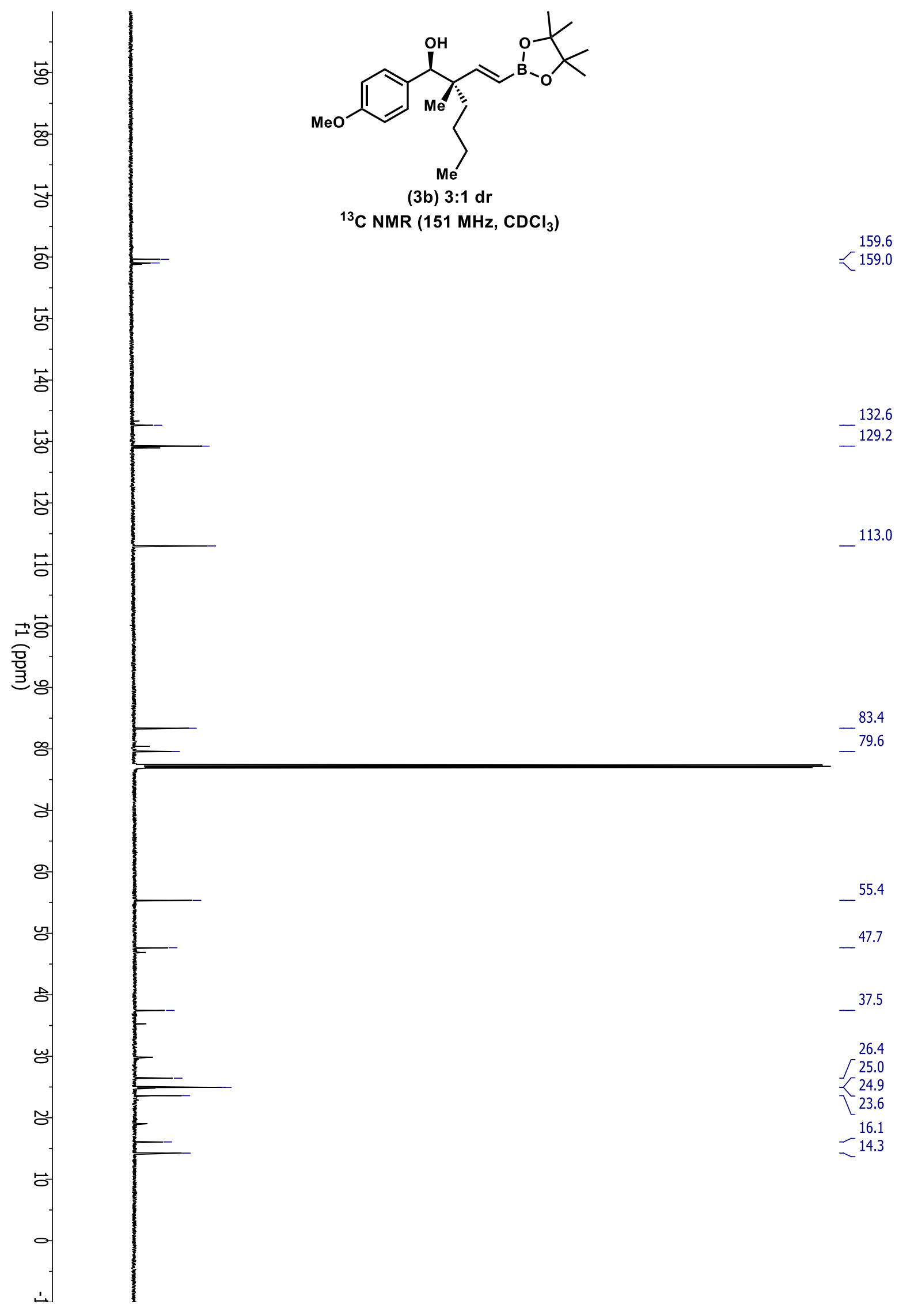




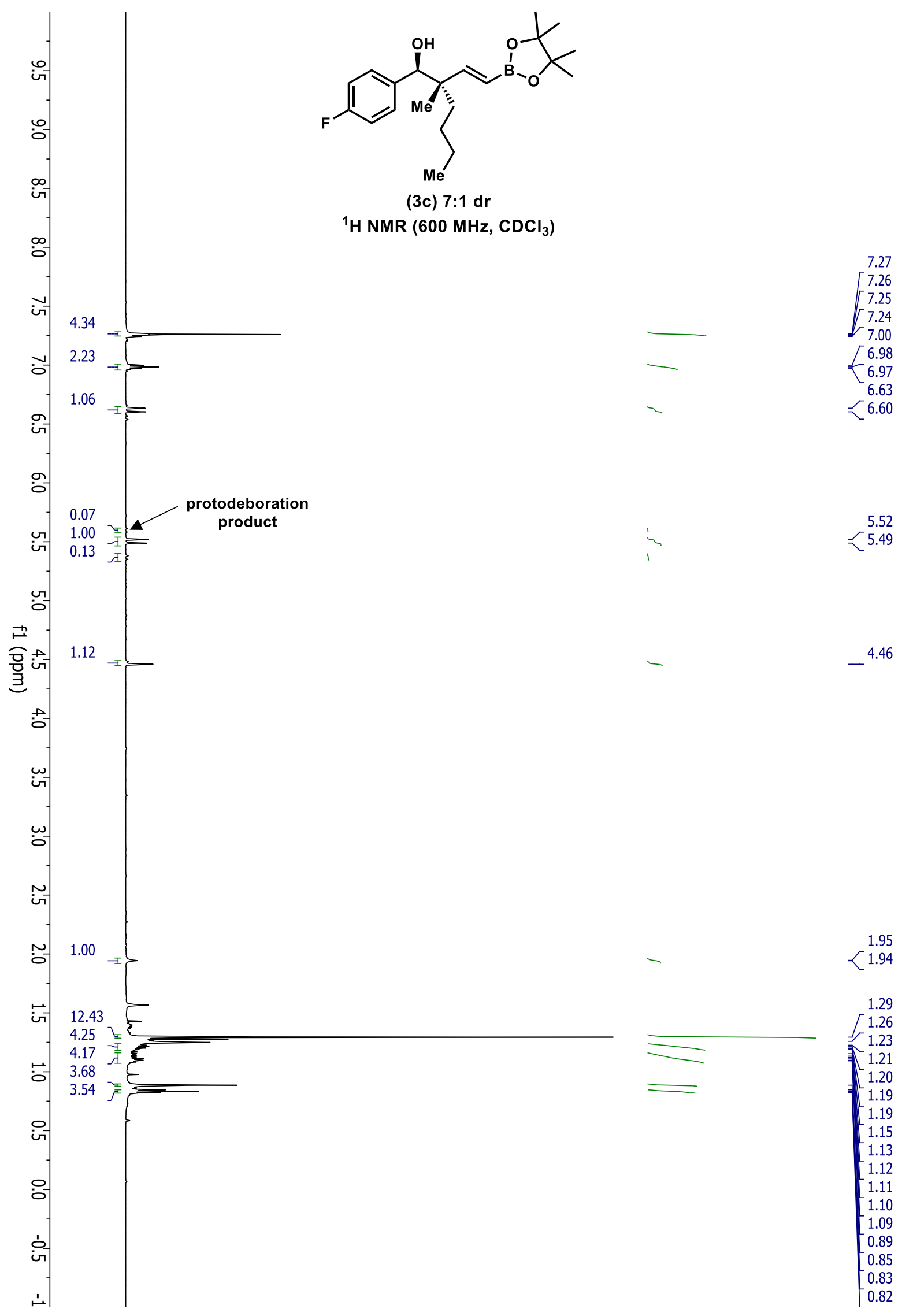




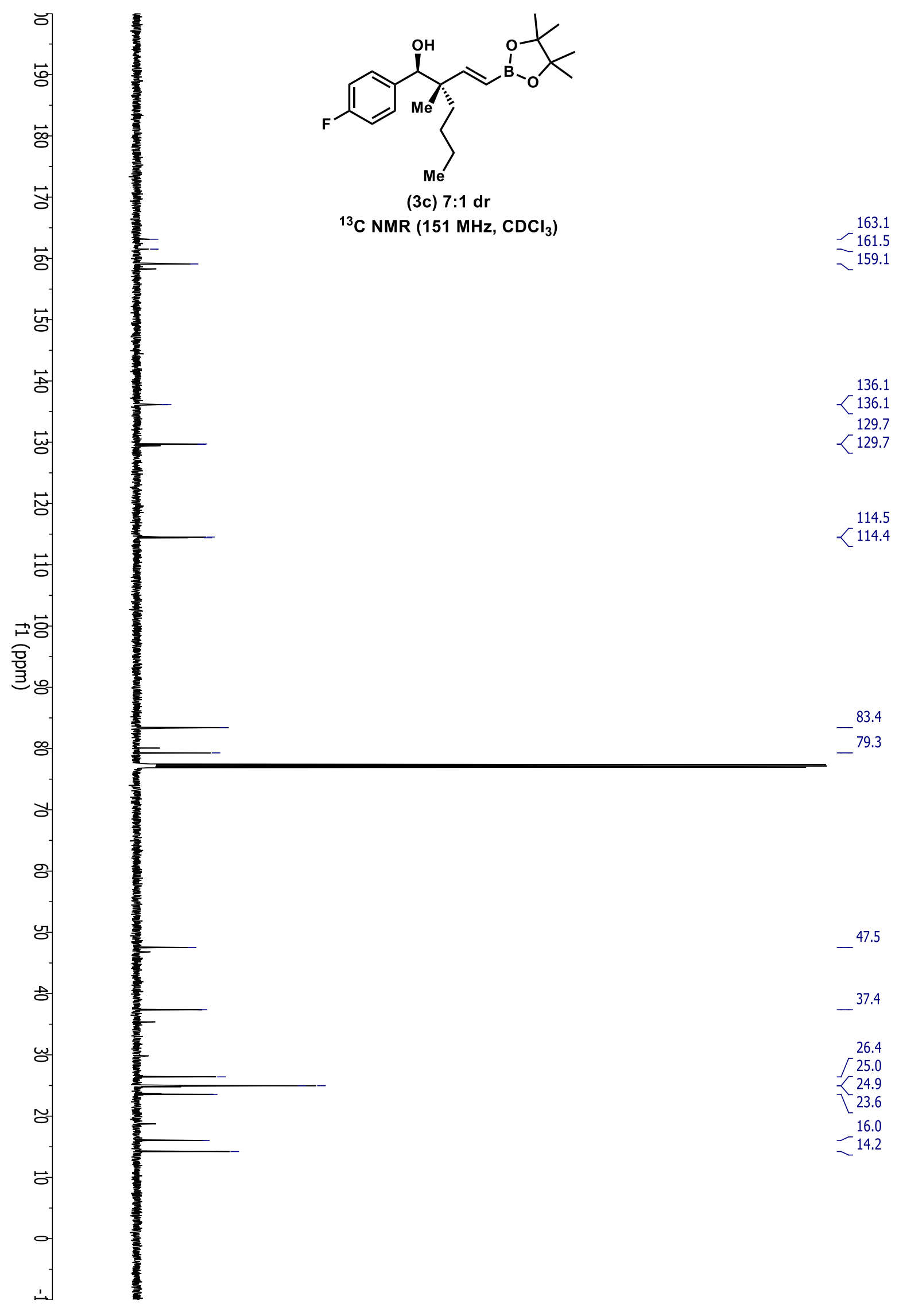




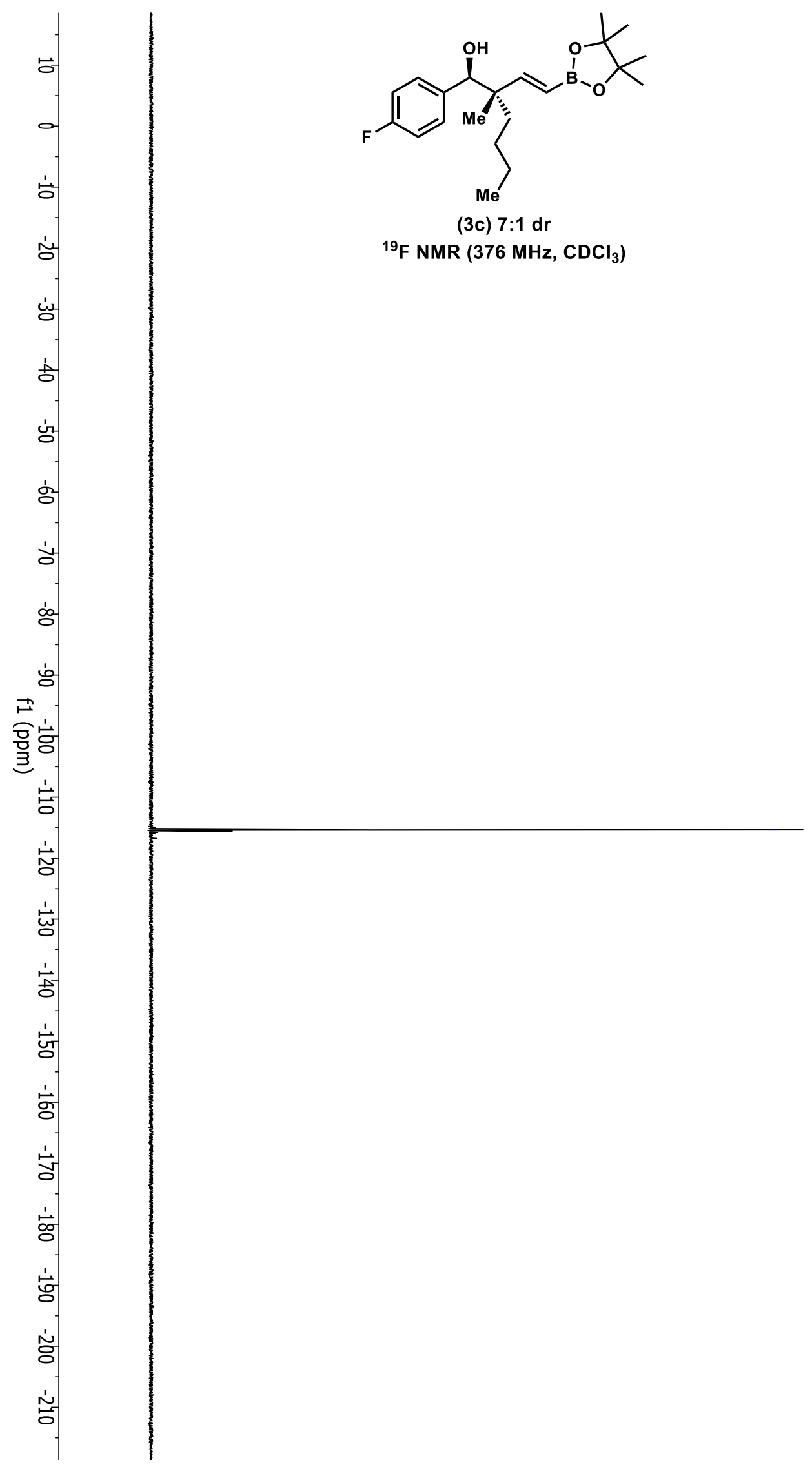

$-115.4$ 


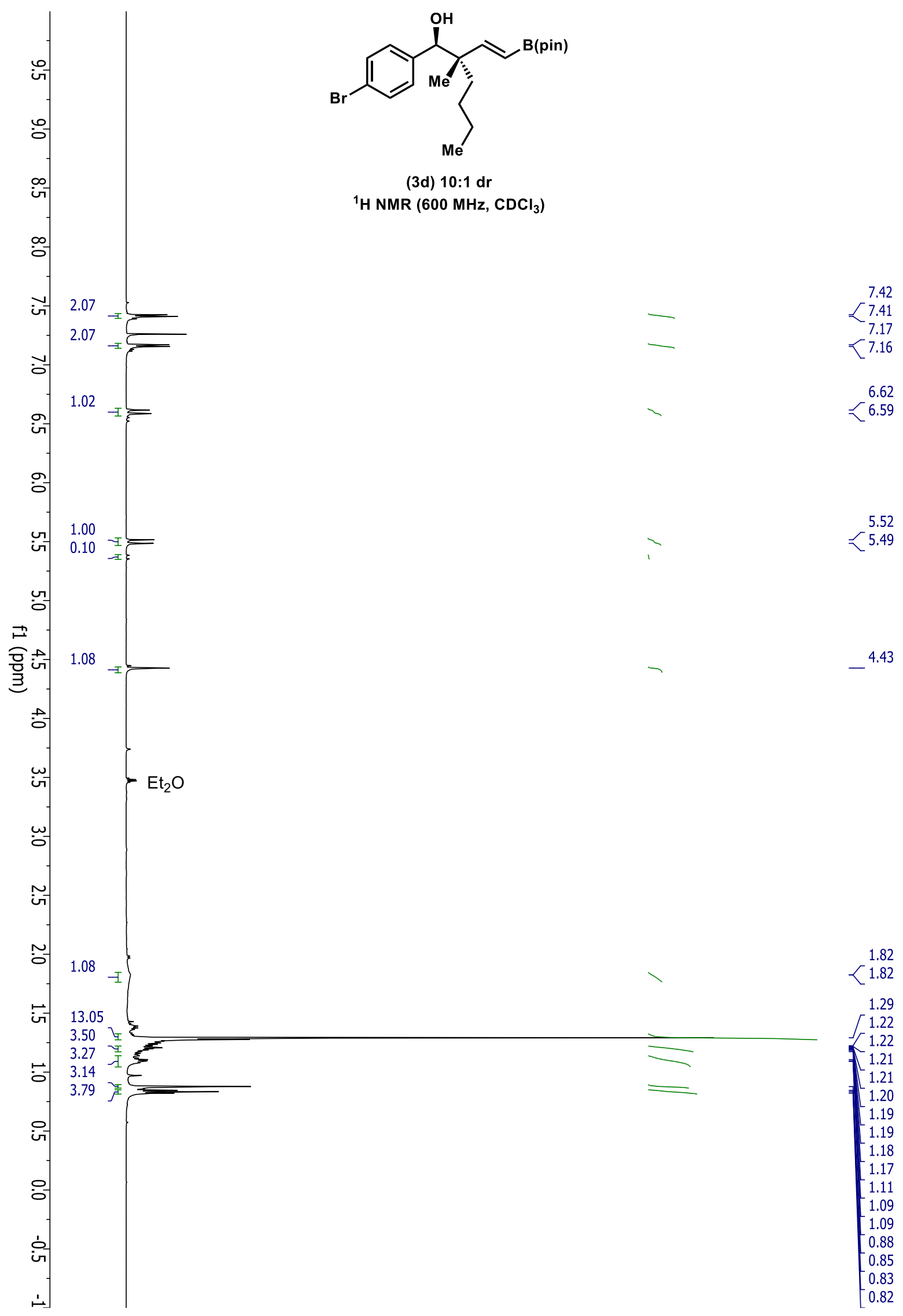




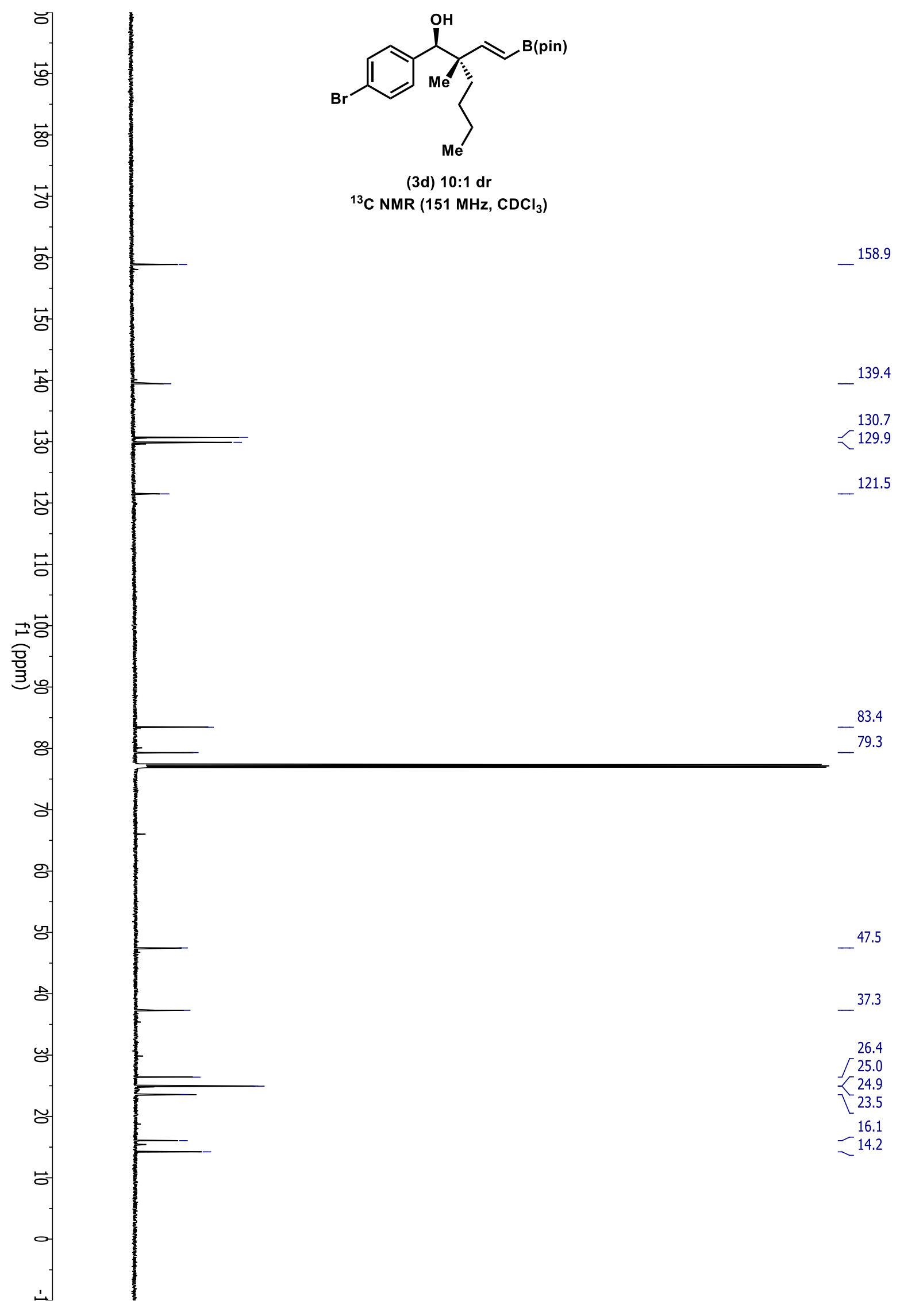



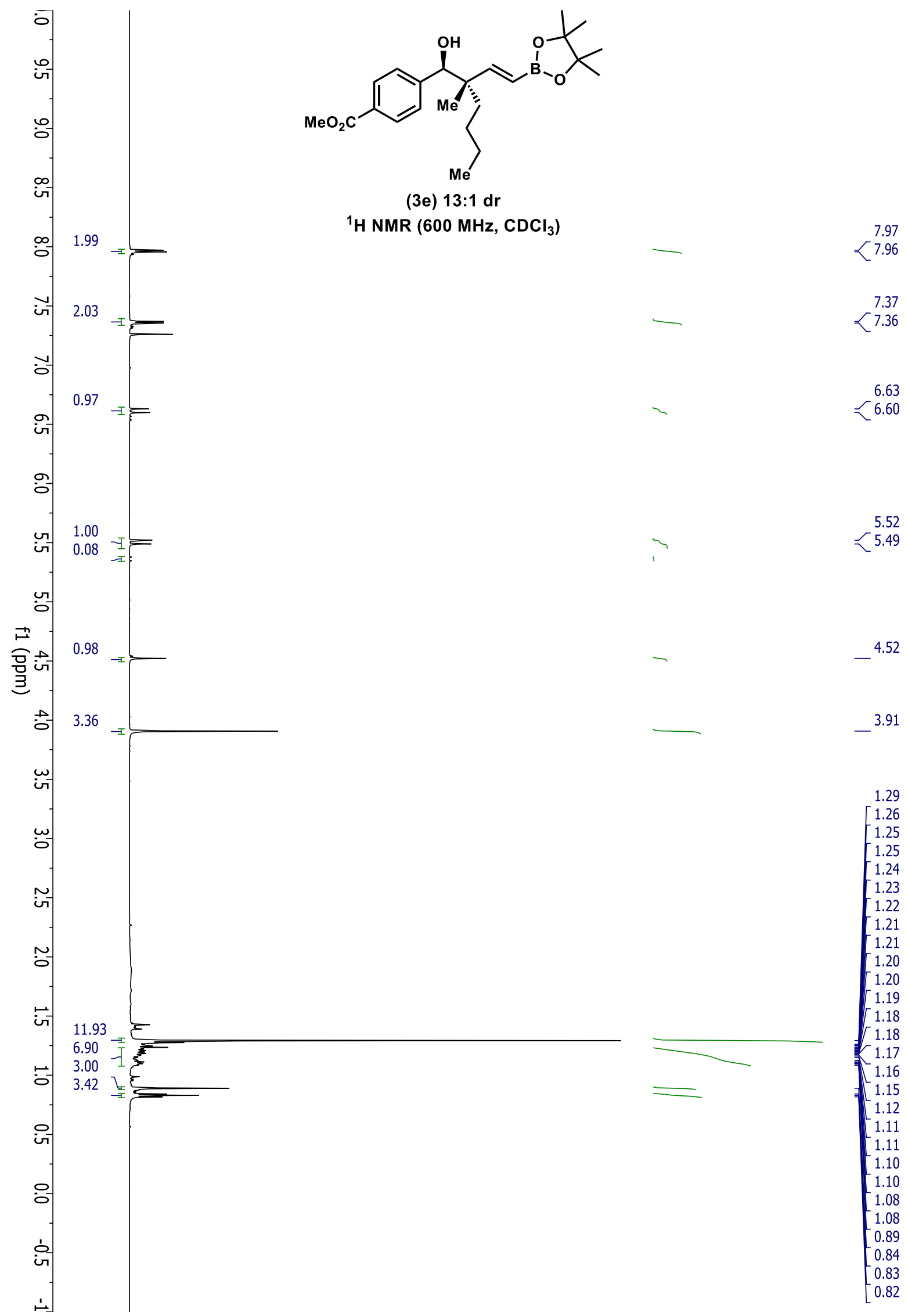


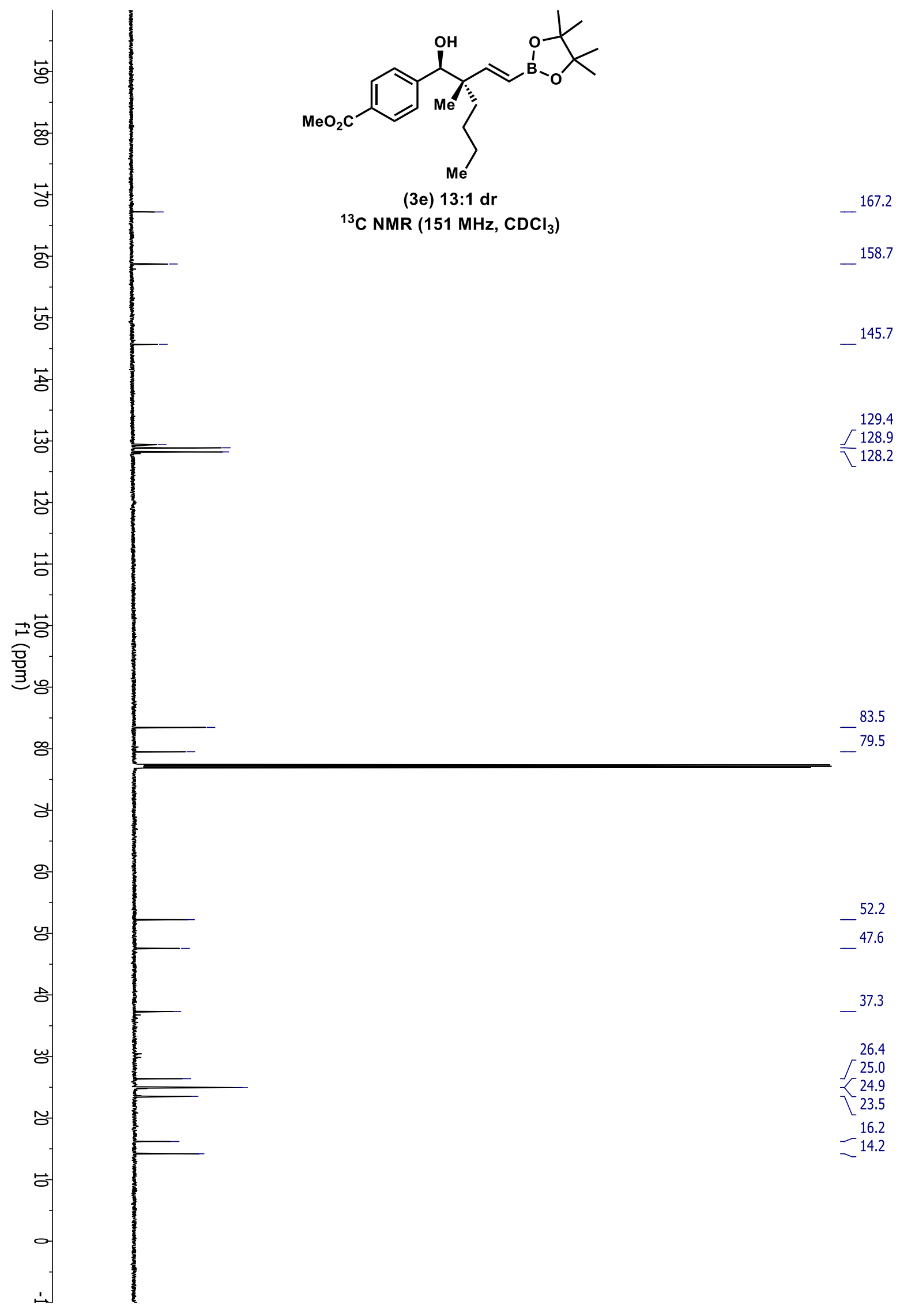




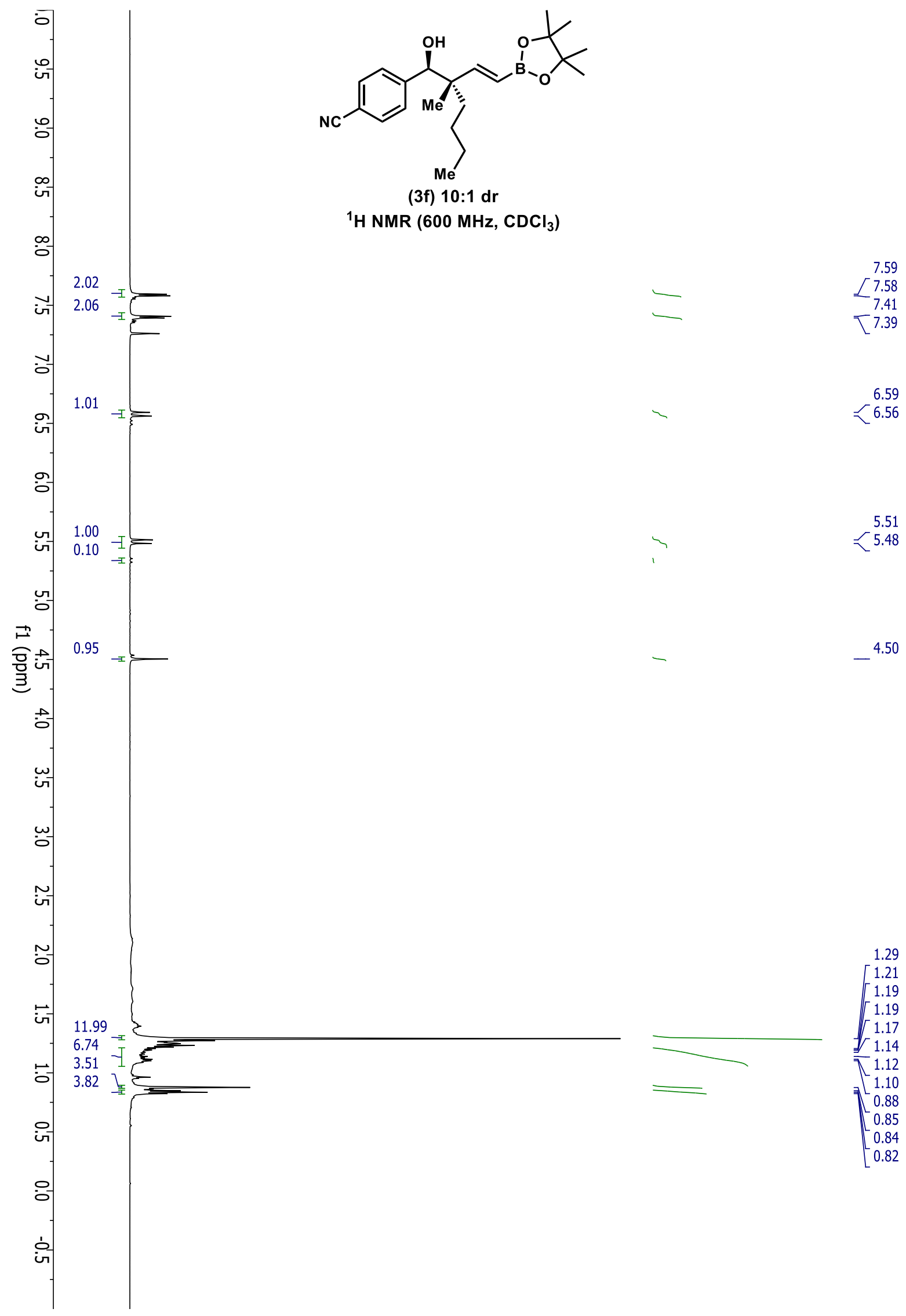




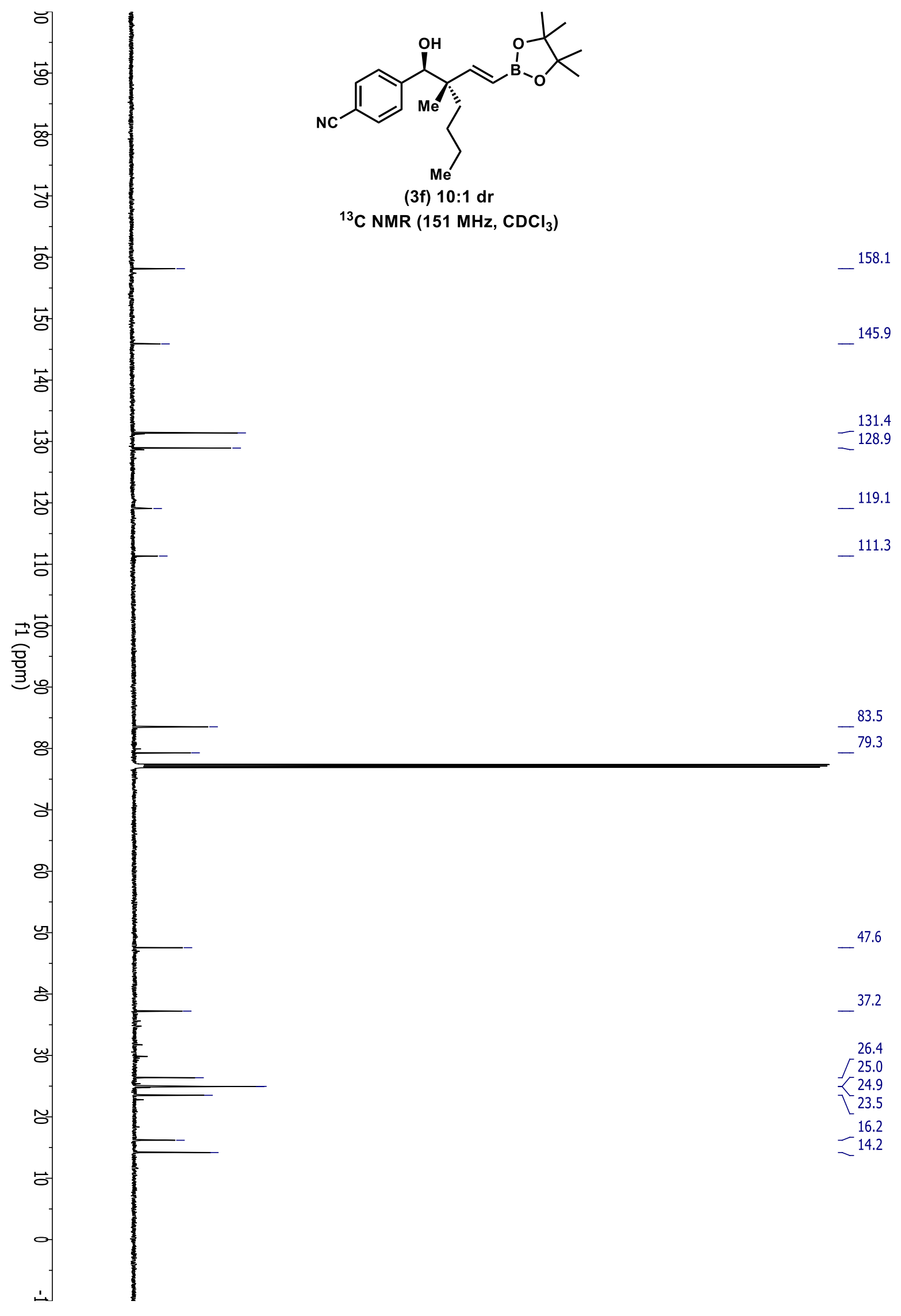




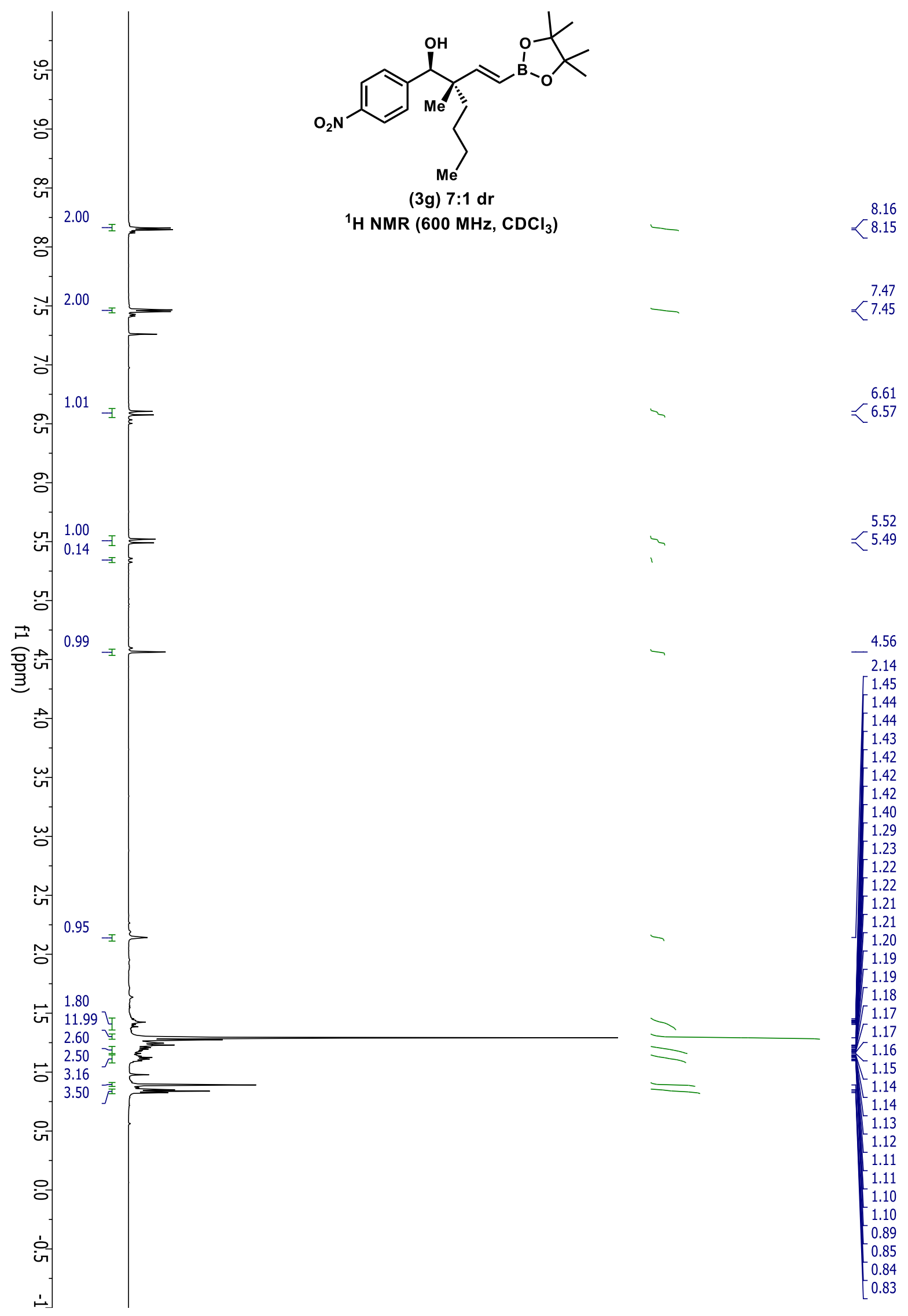




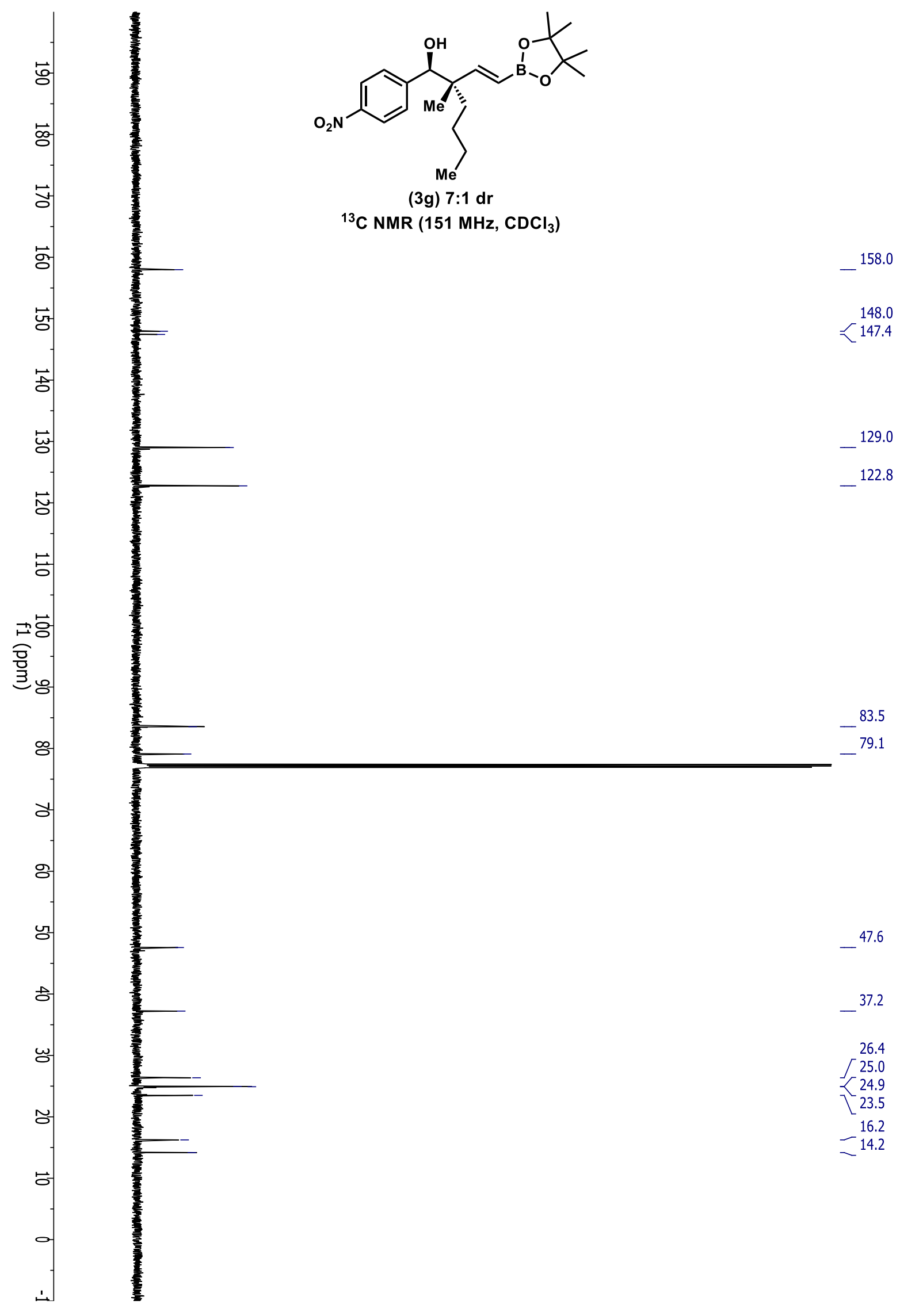




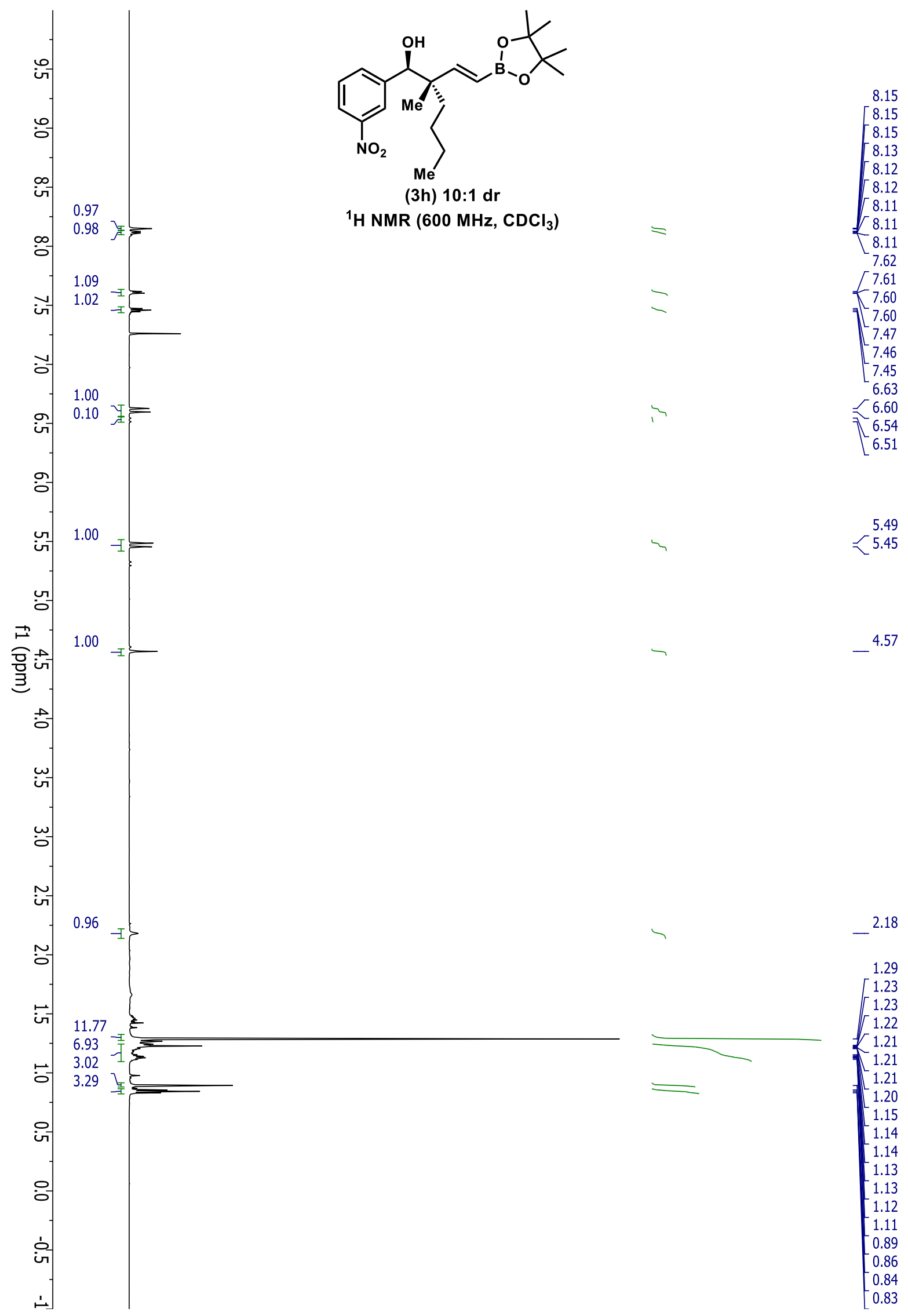




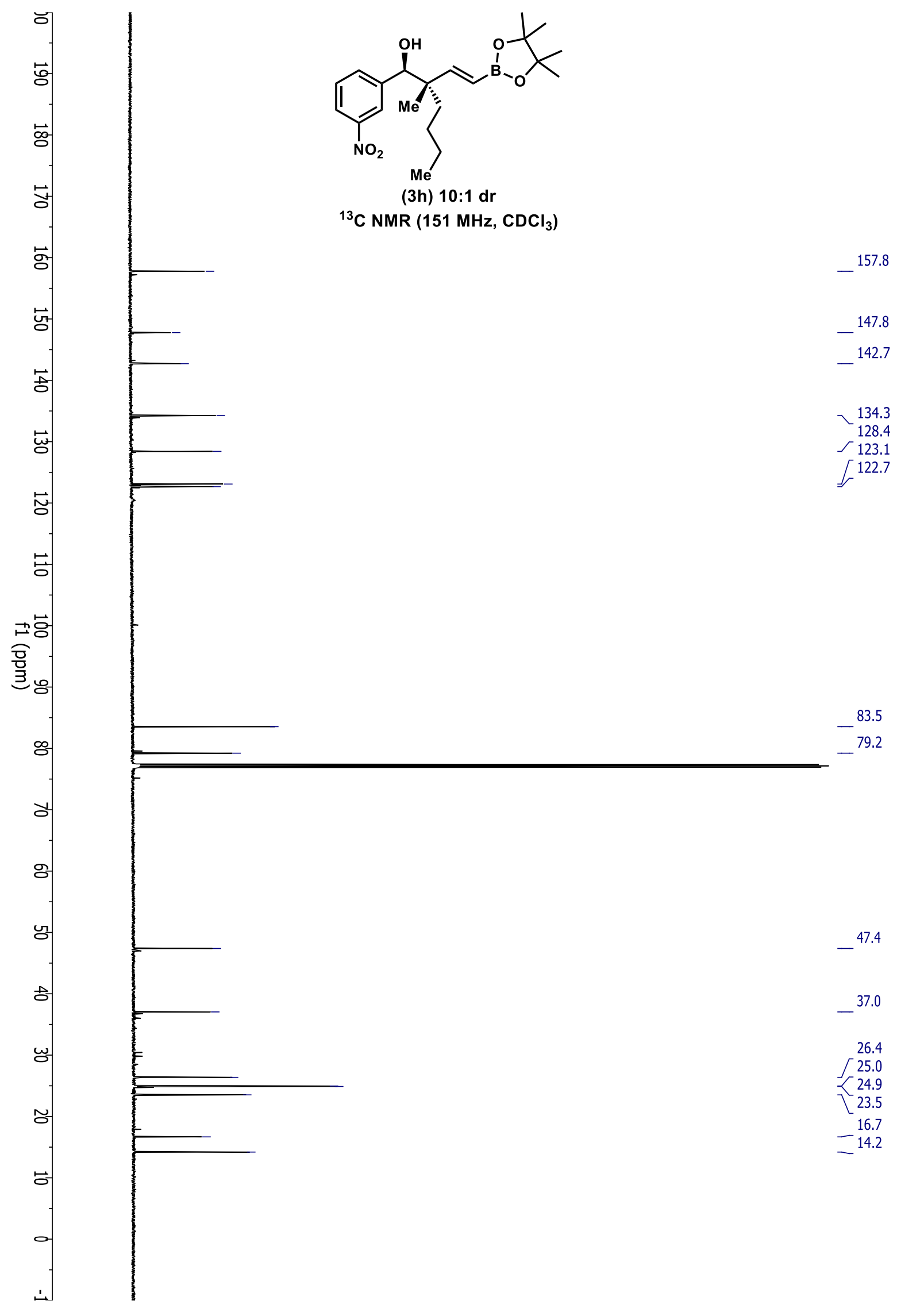




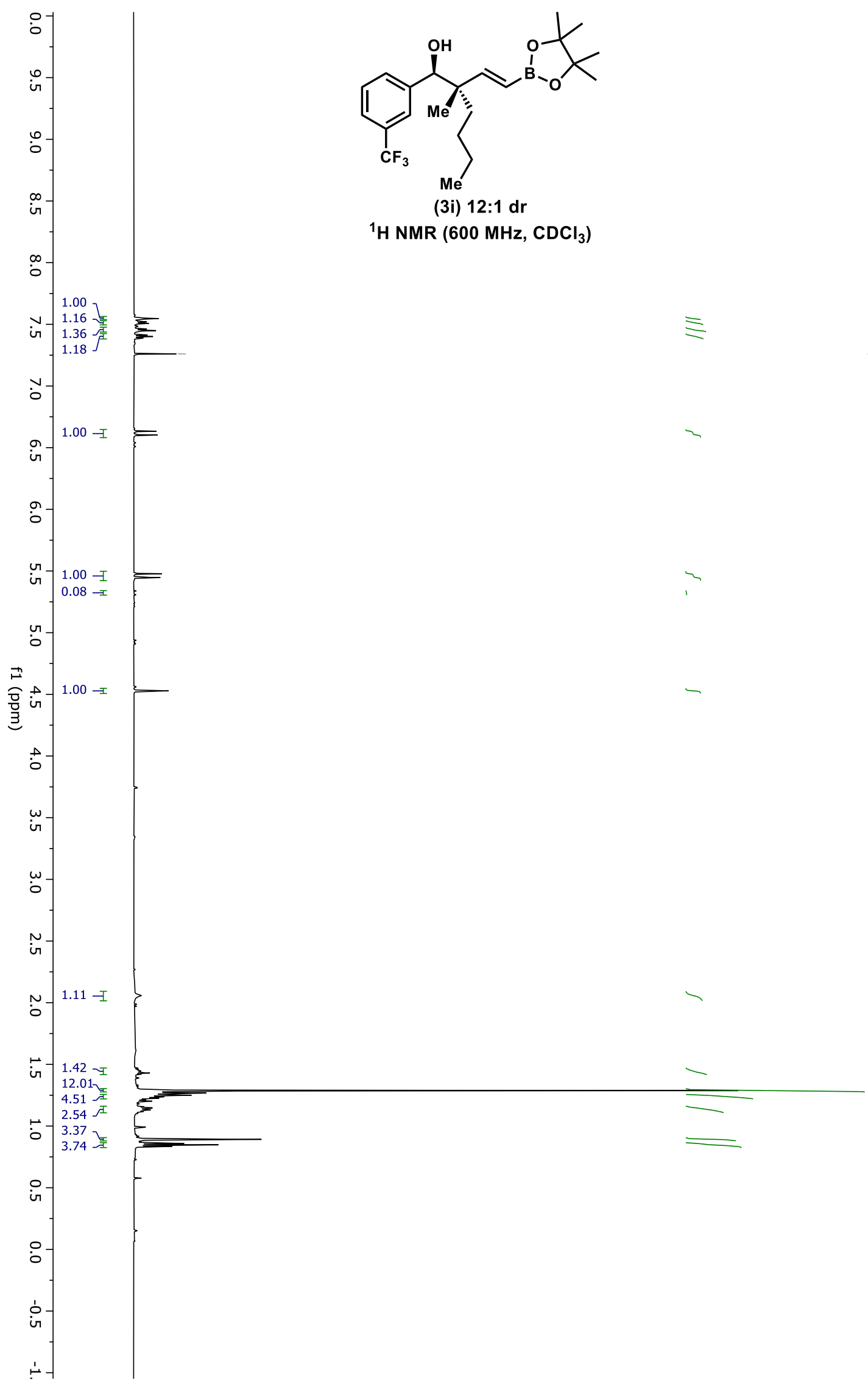




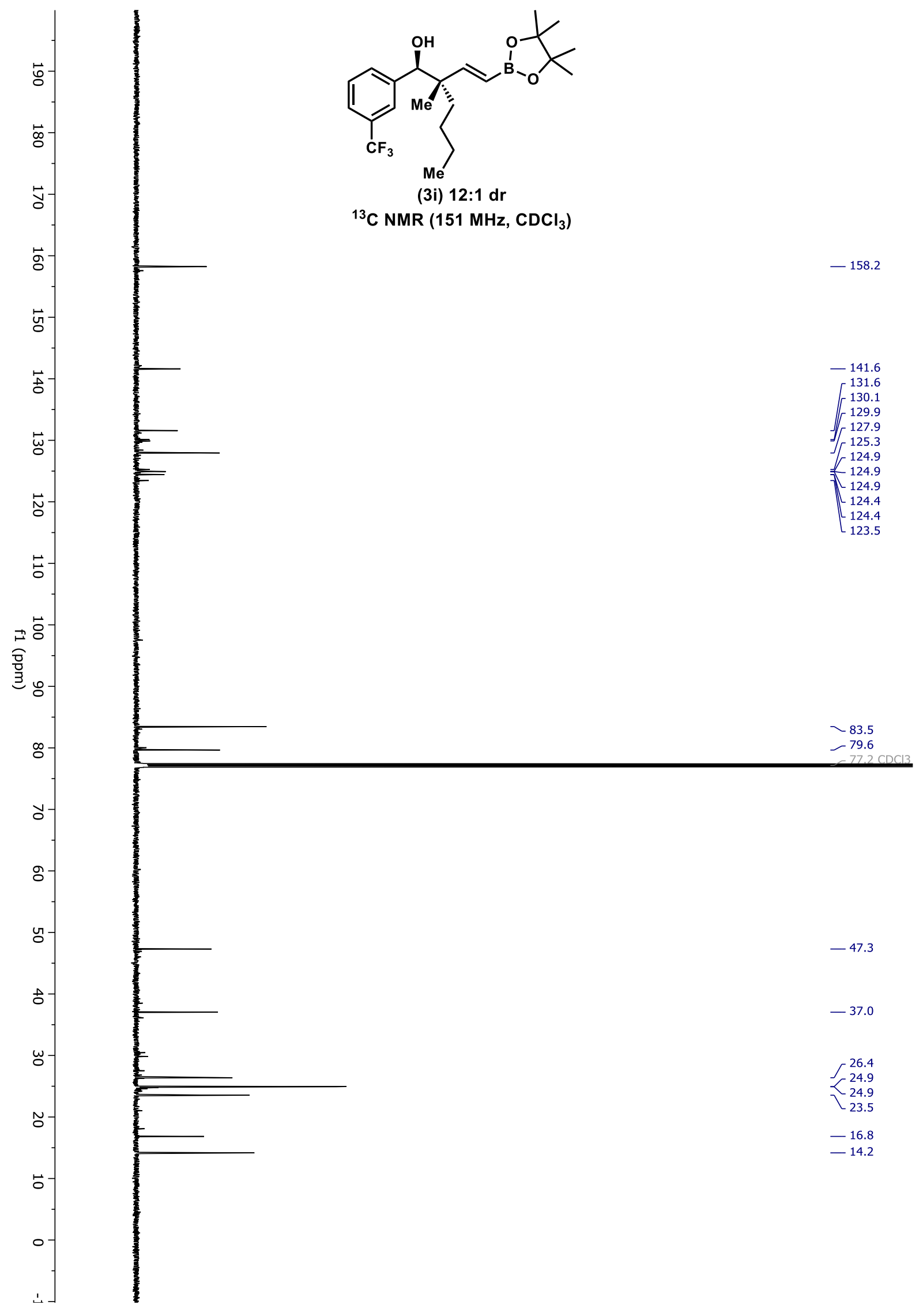



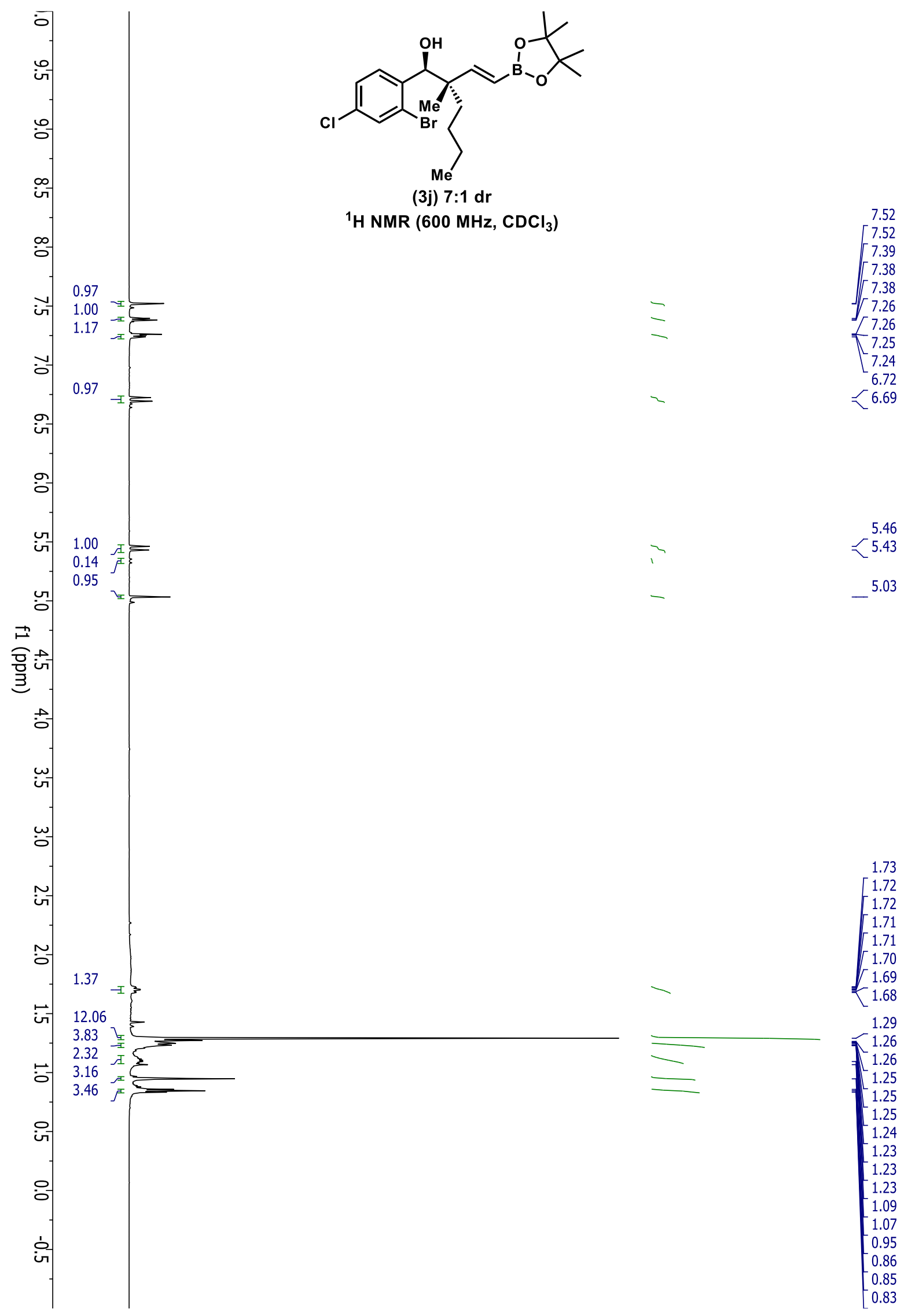


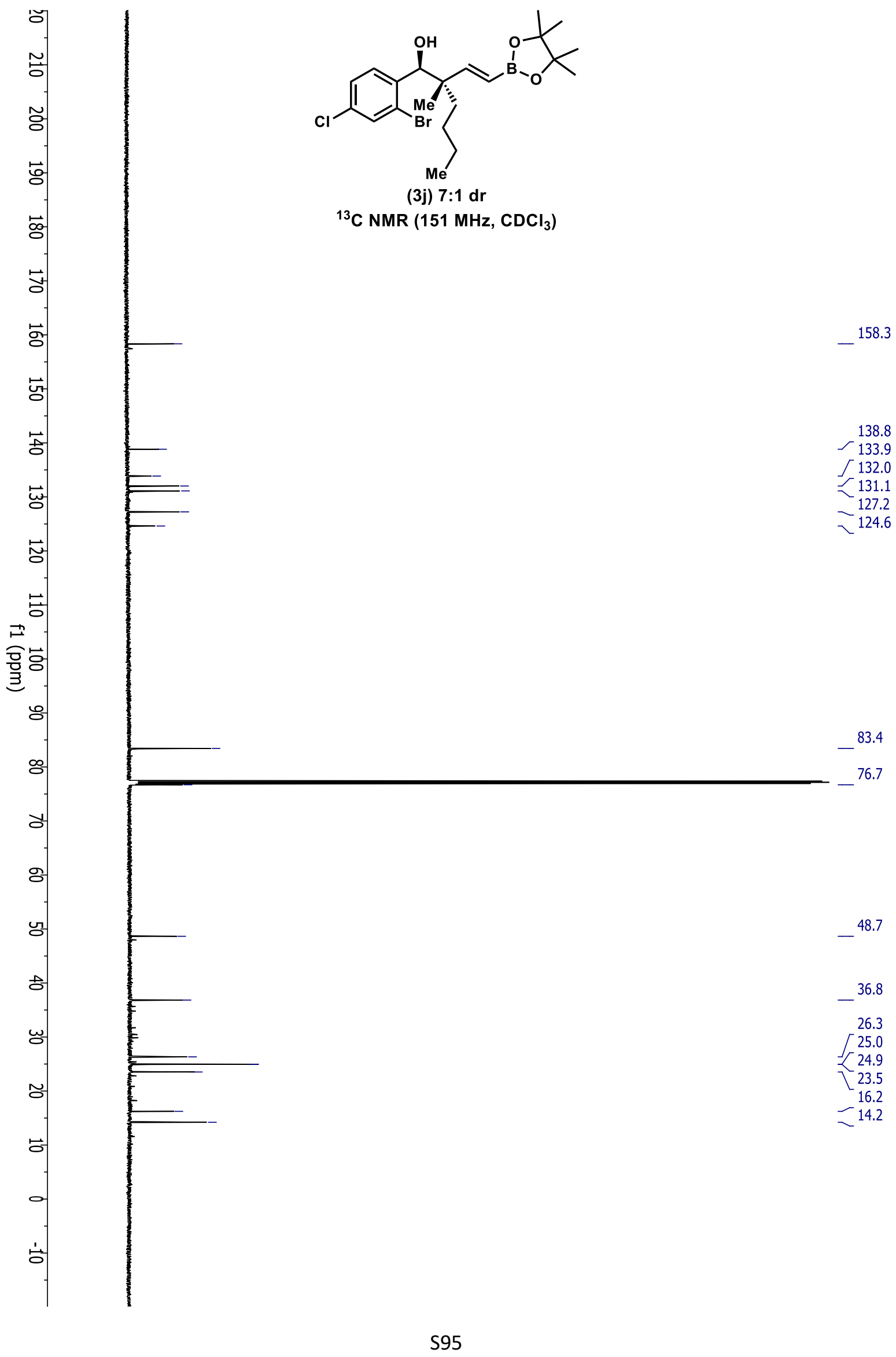



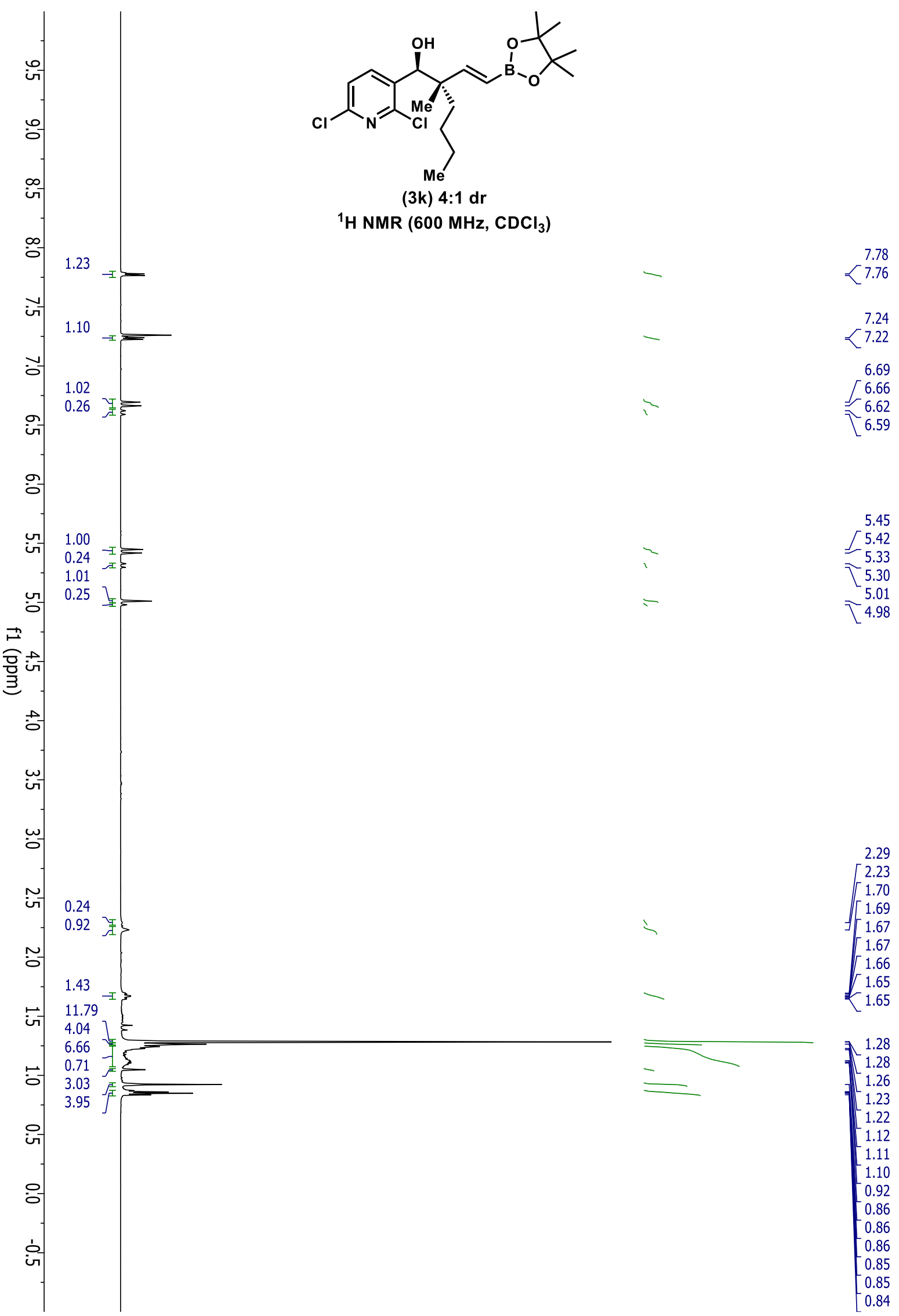


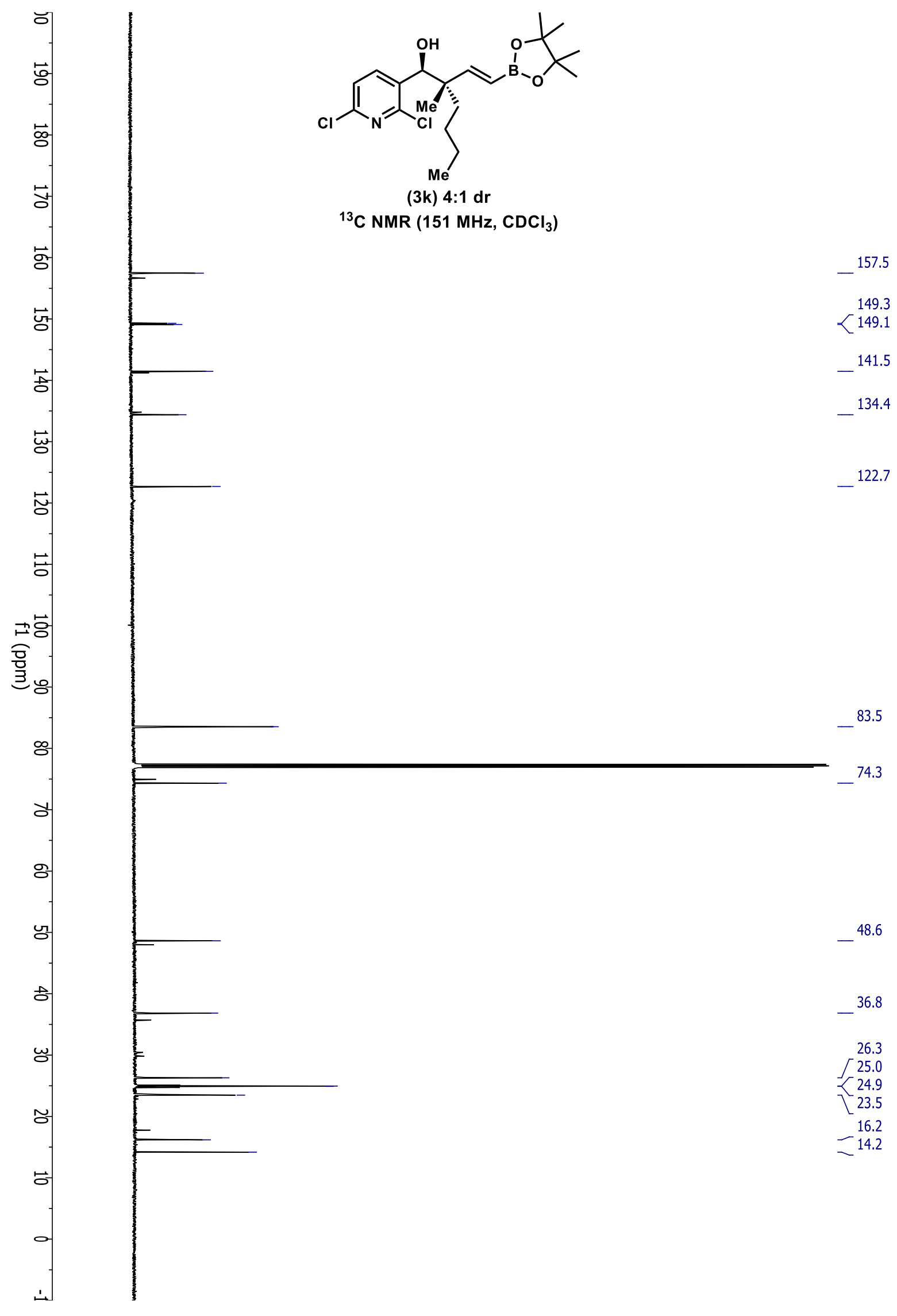




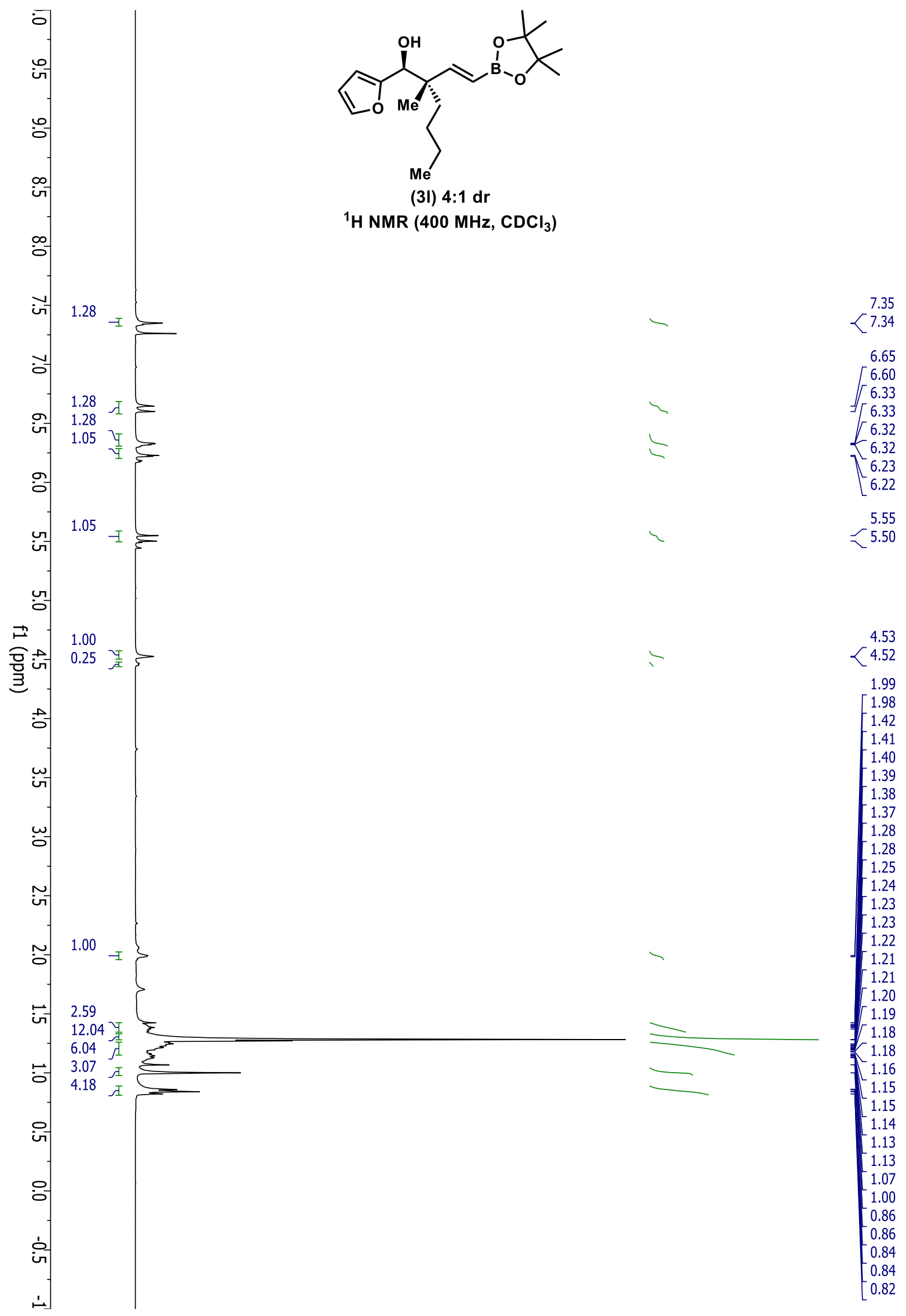




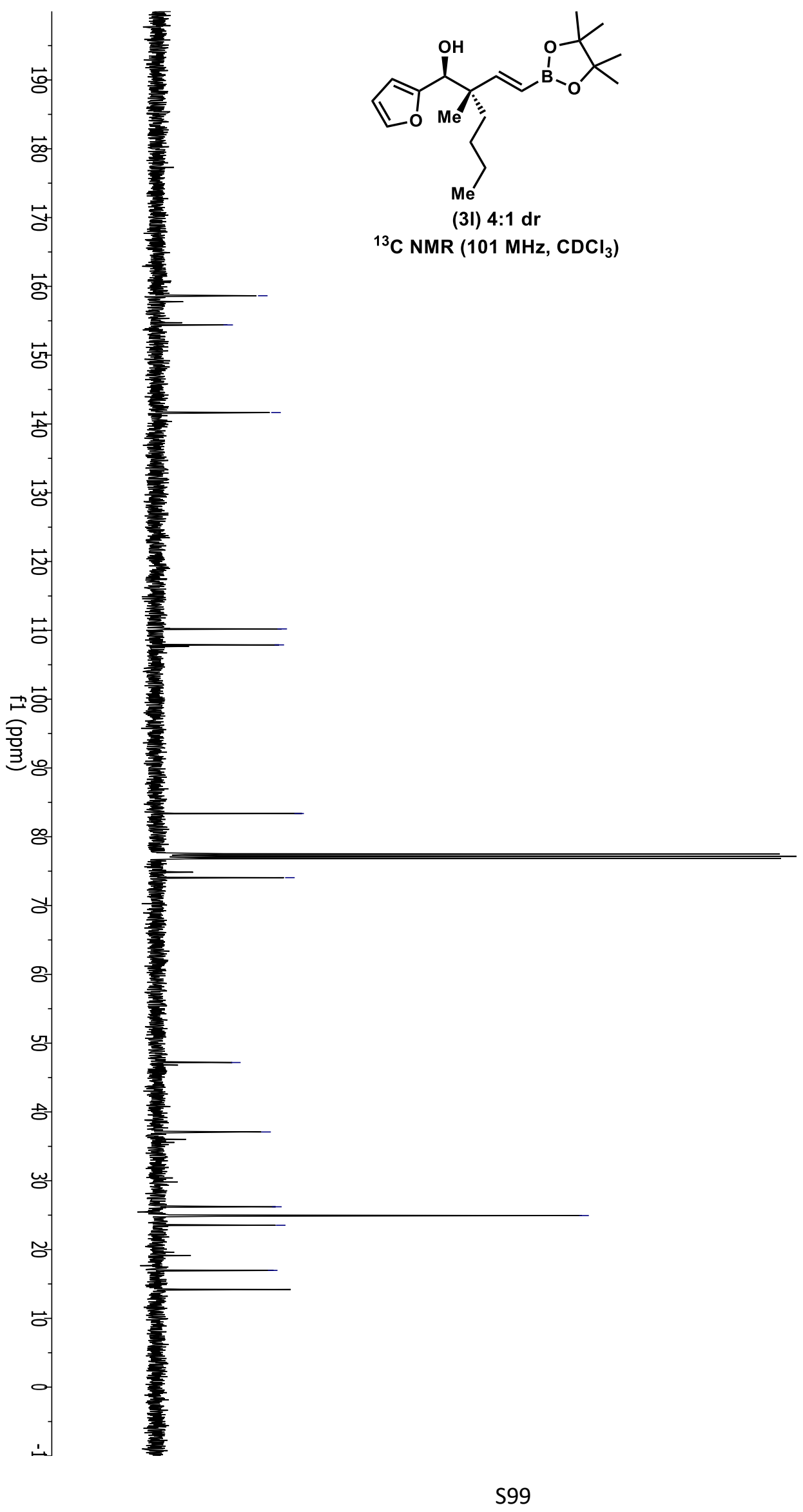

158.6
-154.4

${ }^{-141.7}$

110.2
-107.9

$-83.4$

74.1

$-47.2$

${ }^{37.1}$

26.2
$\Gamma 24.9$
$\nearrow 23.5$
-17.0

S99 


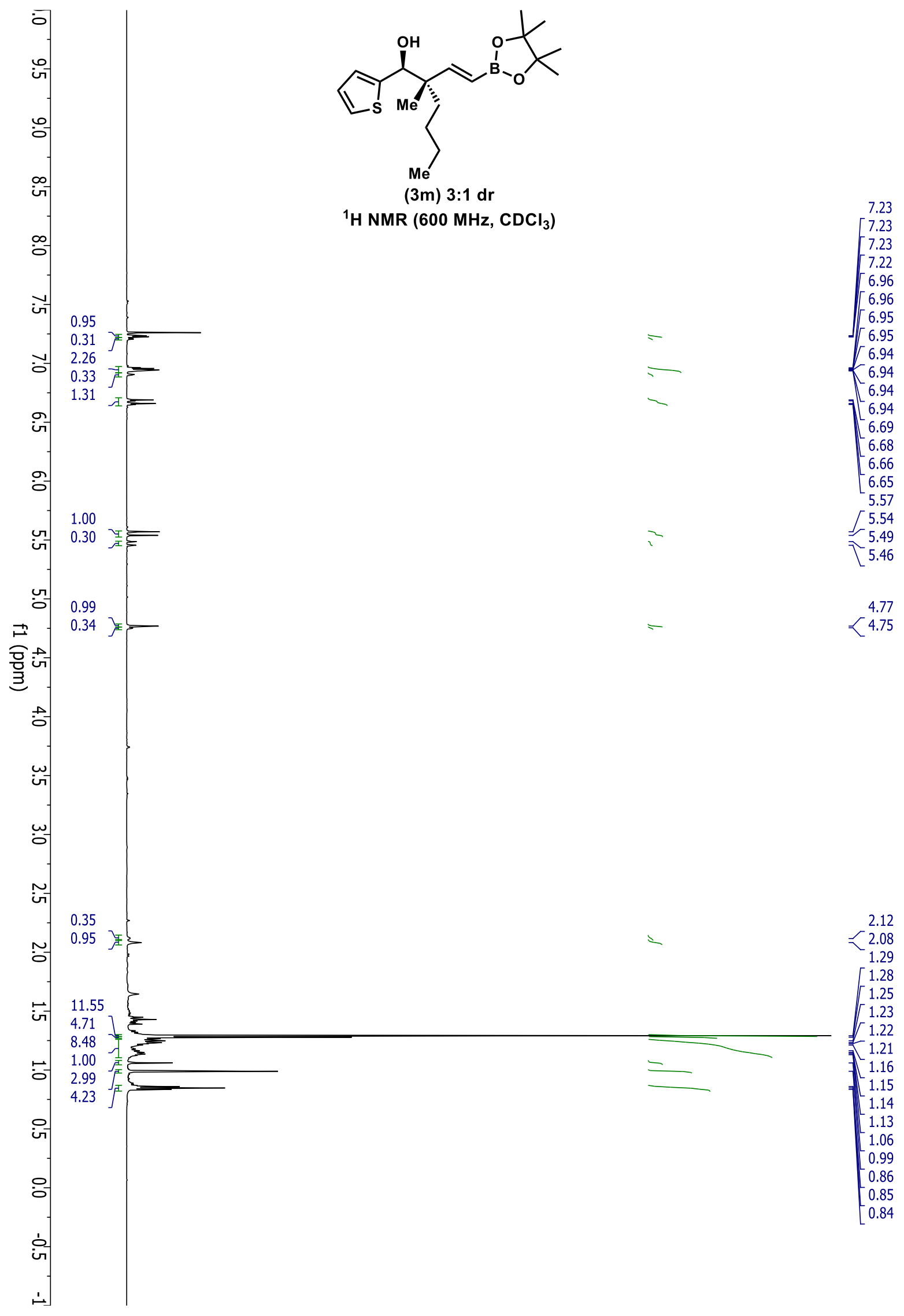




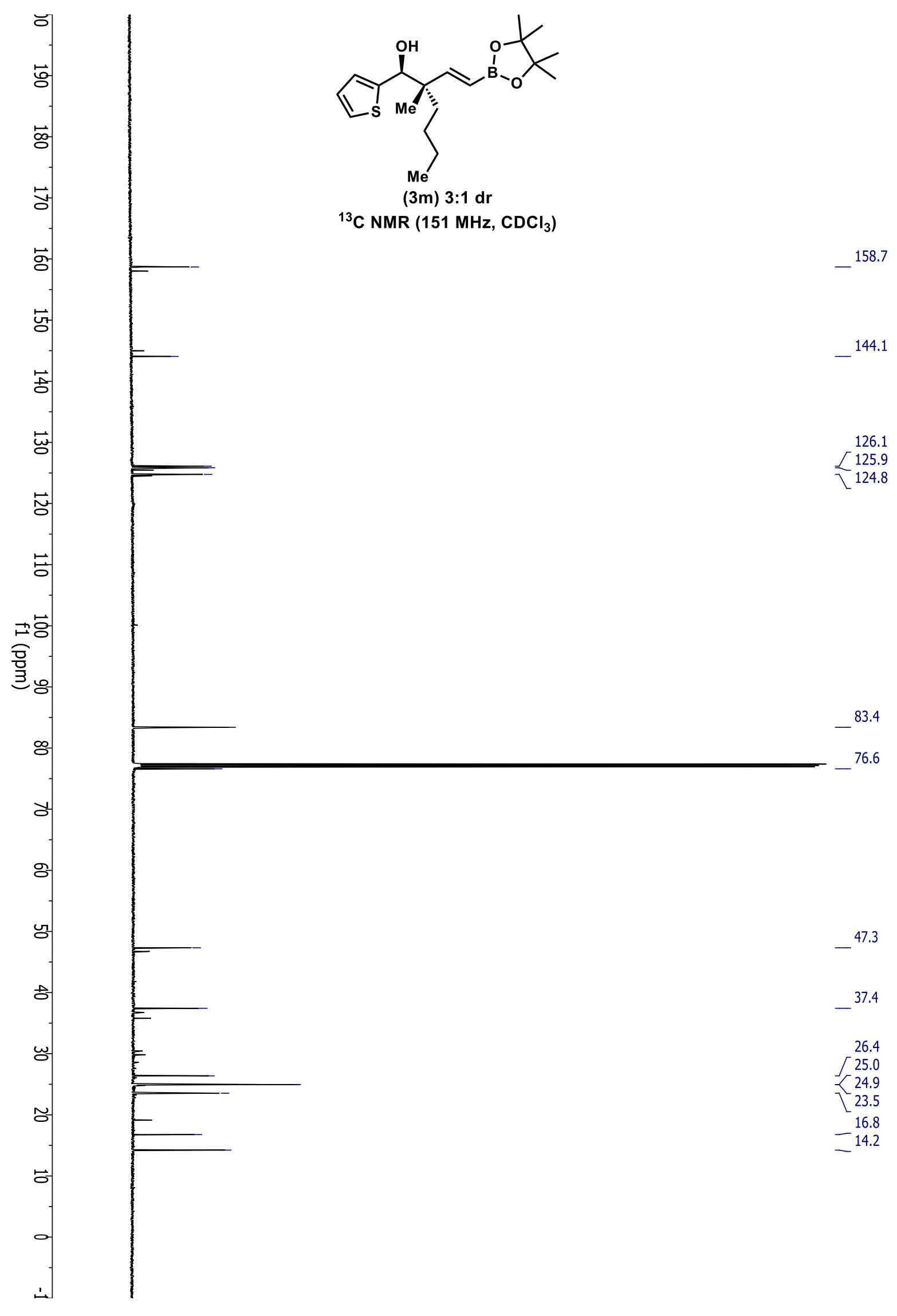




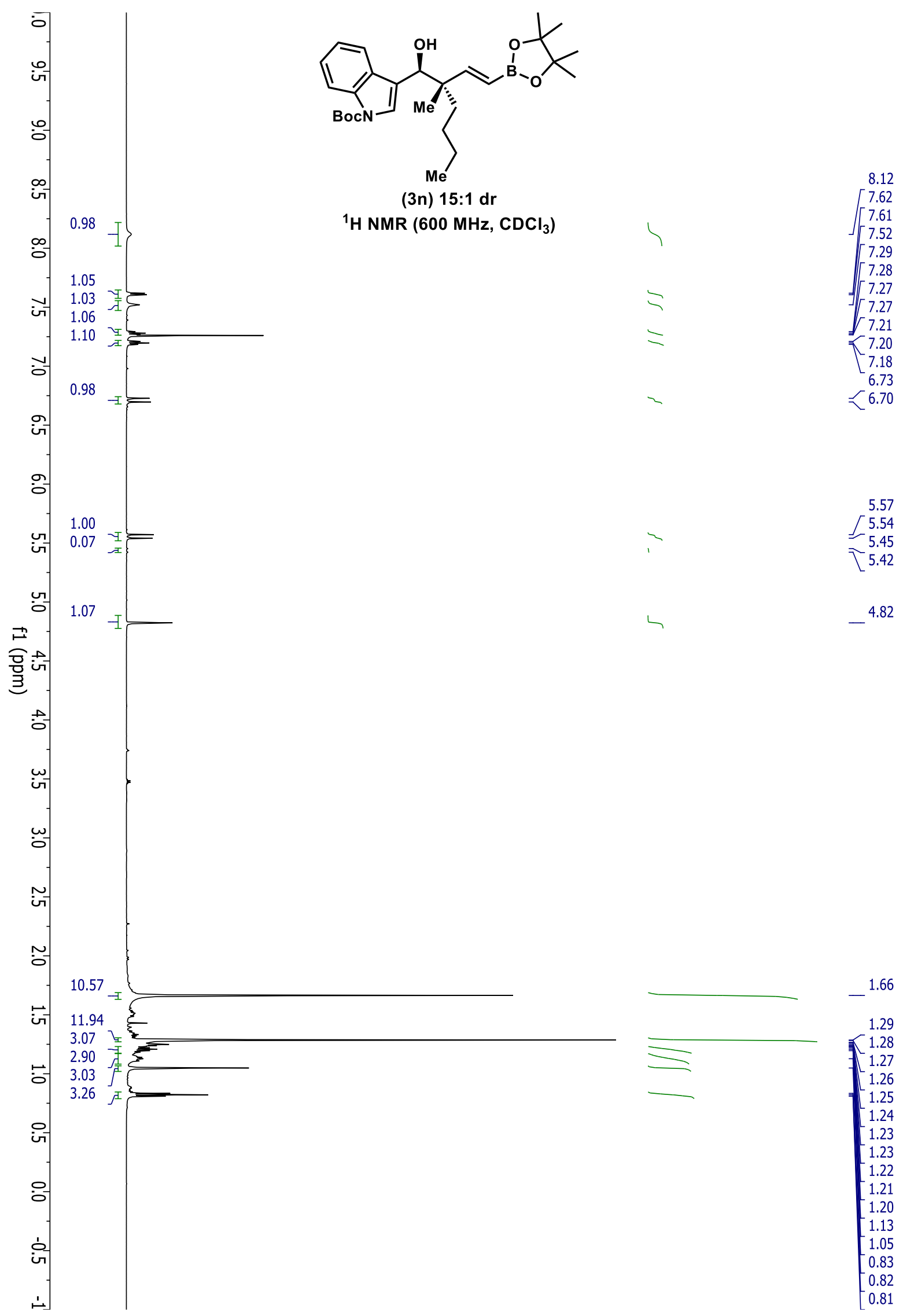




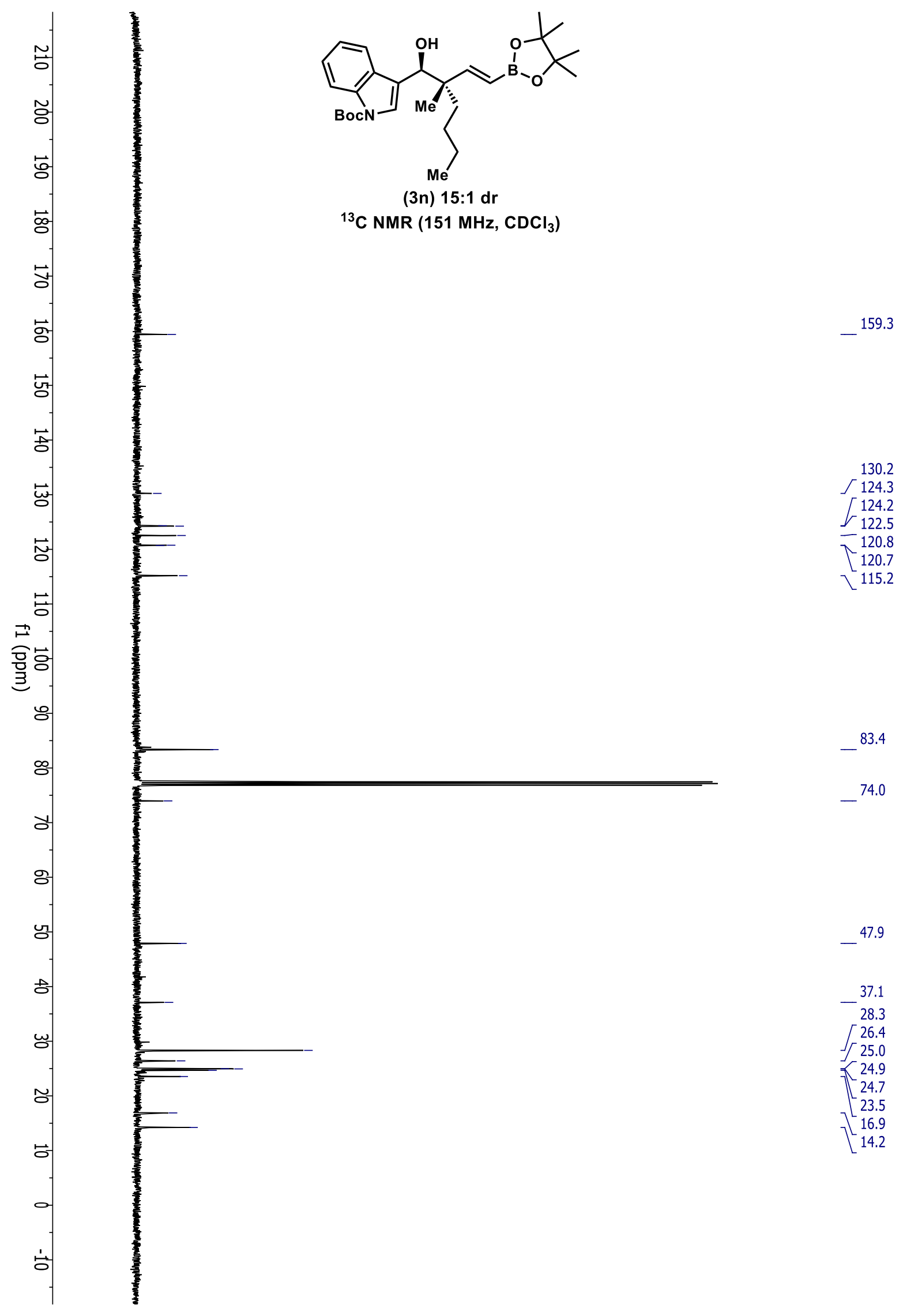




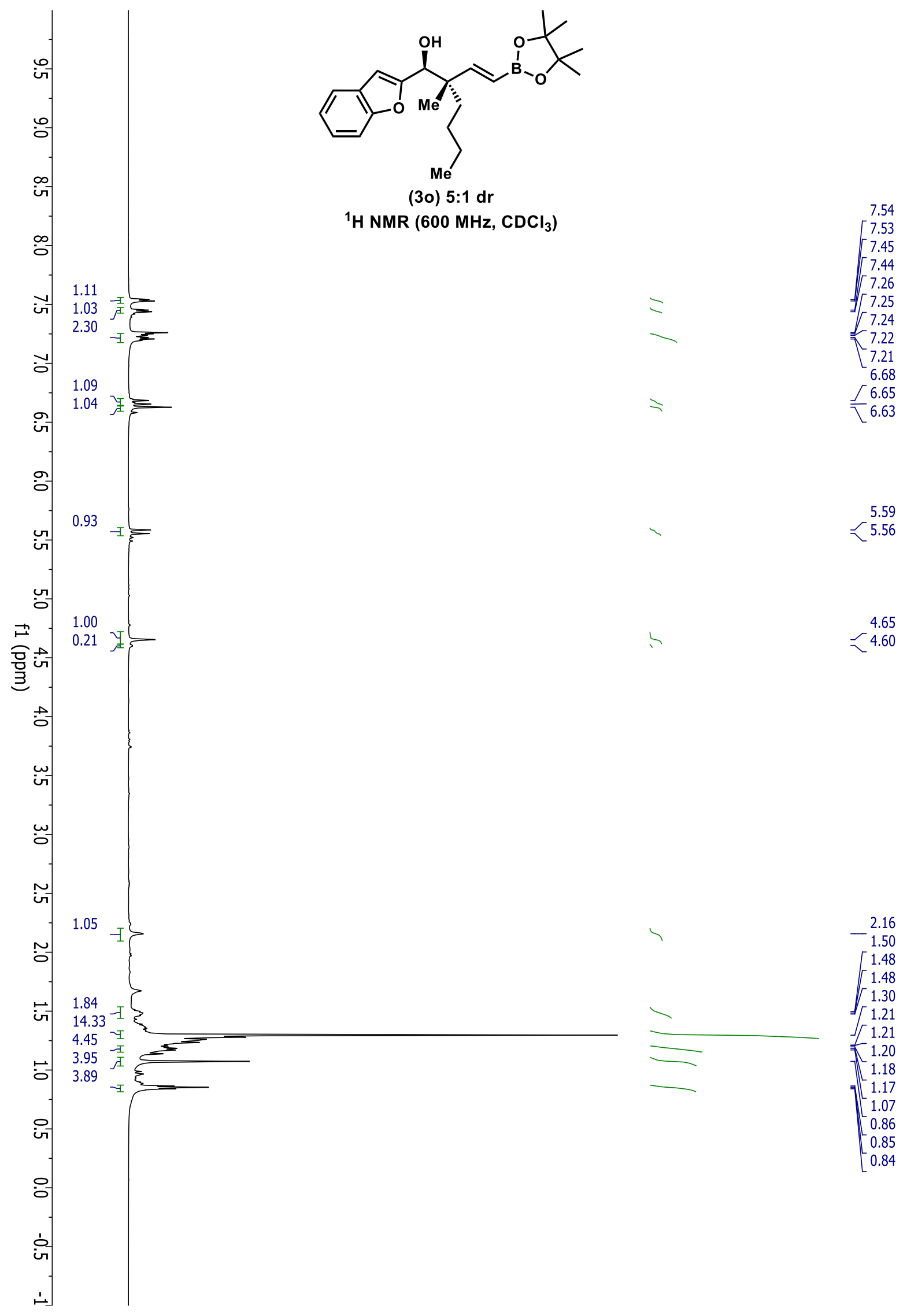




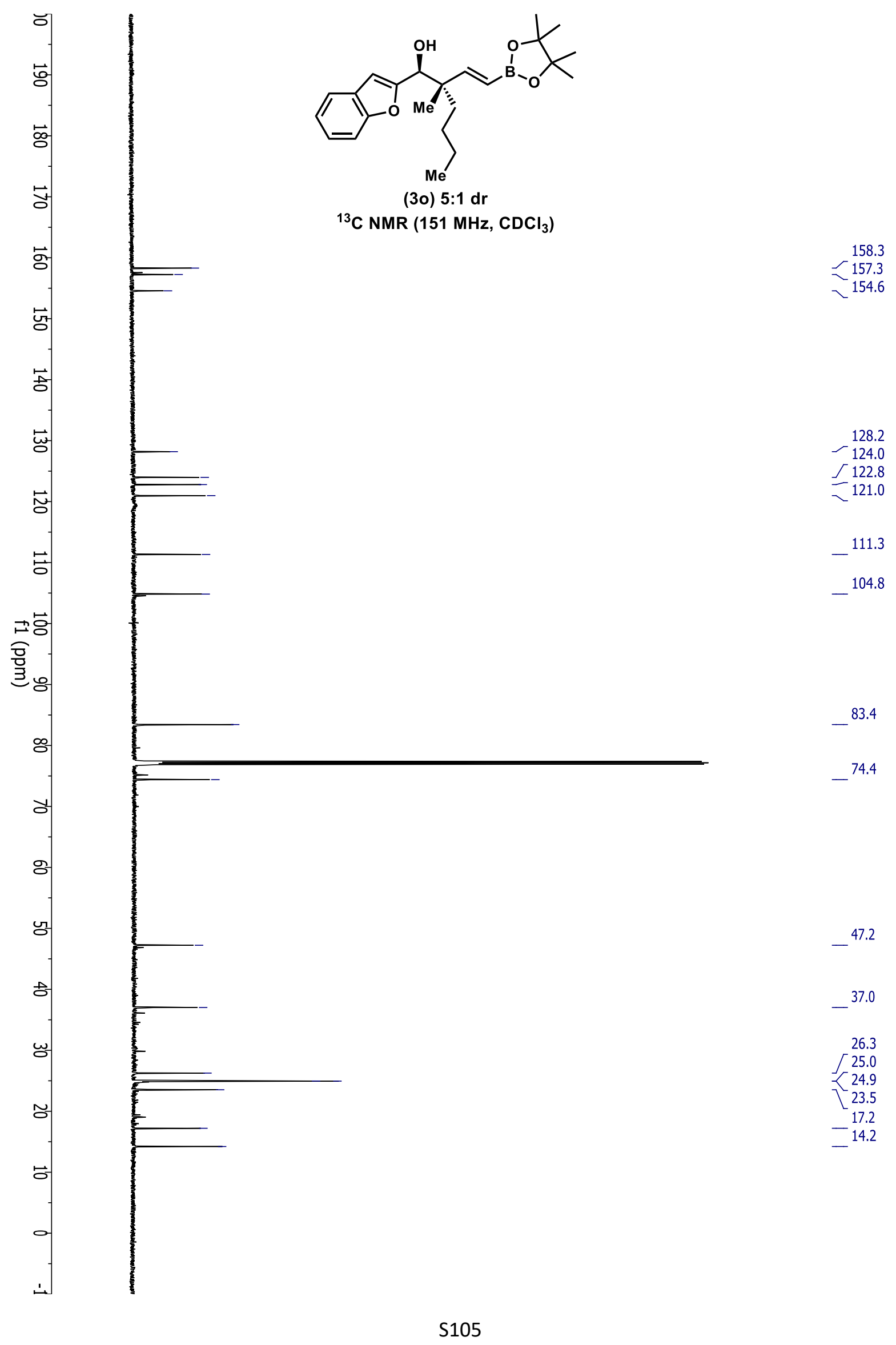




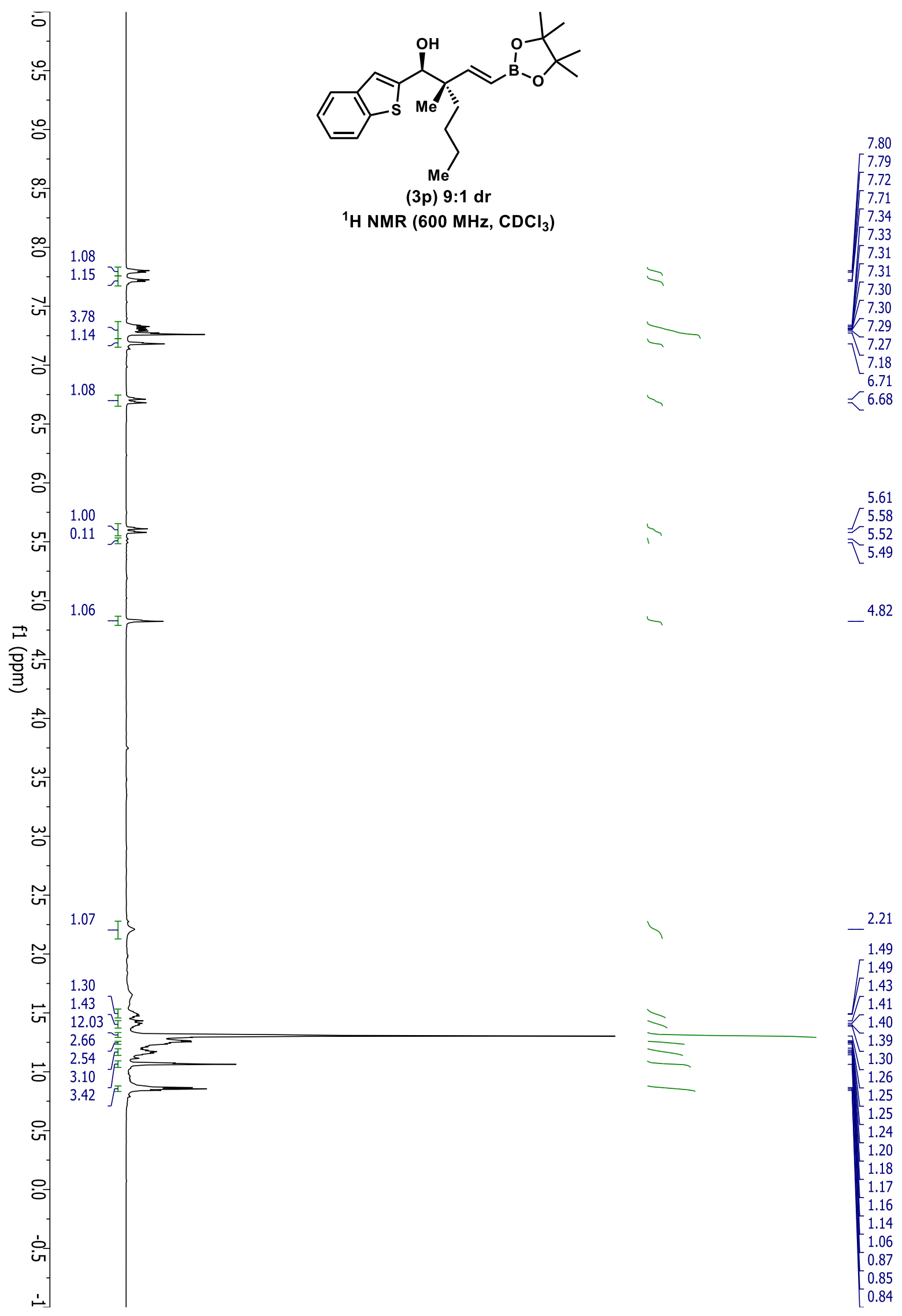




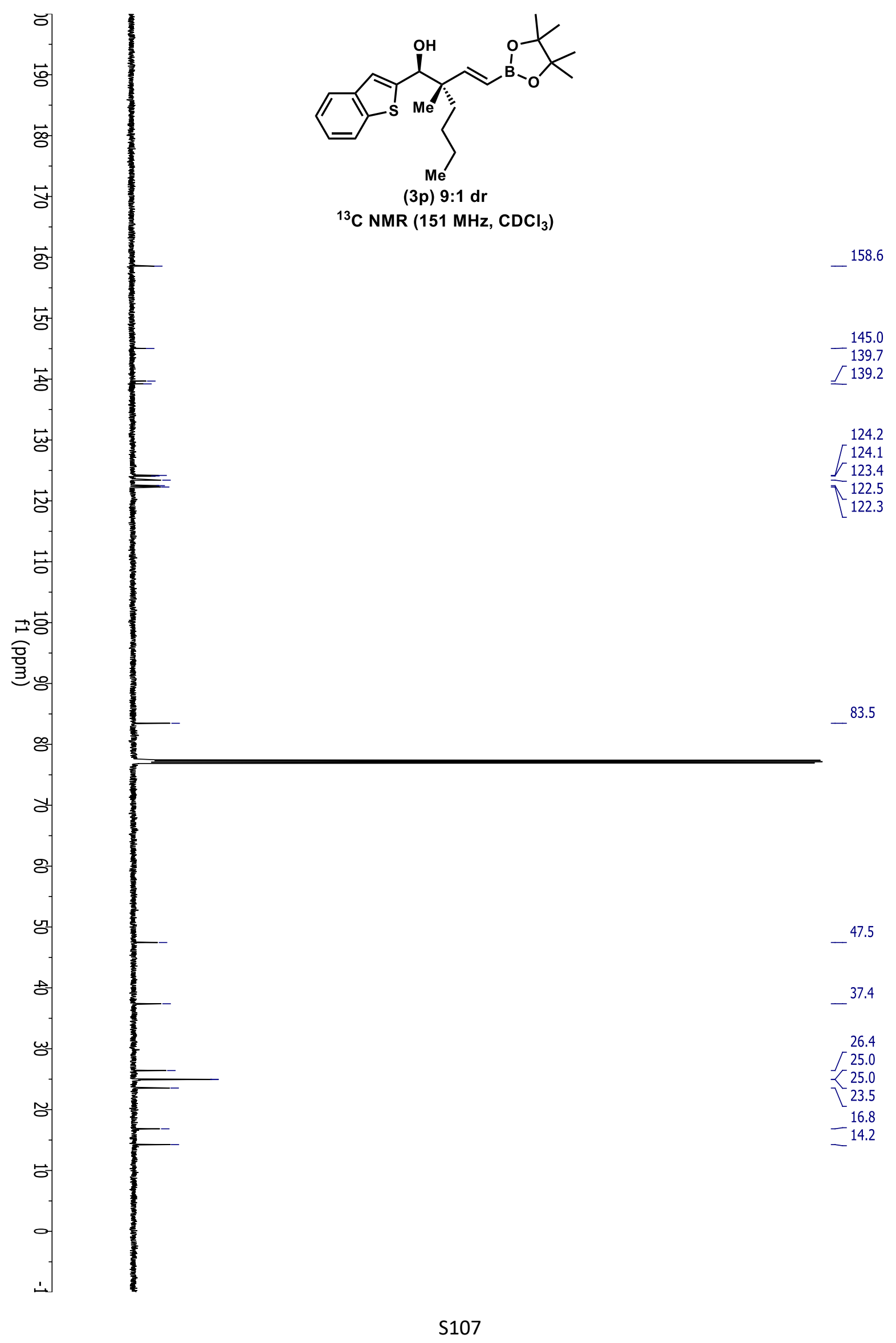




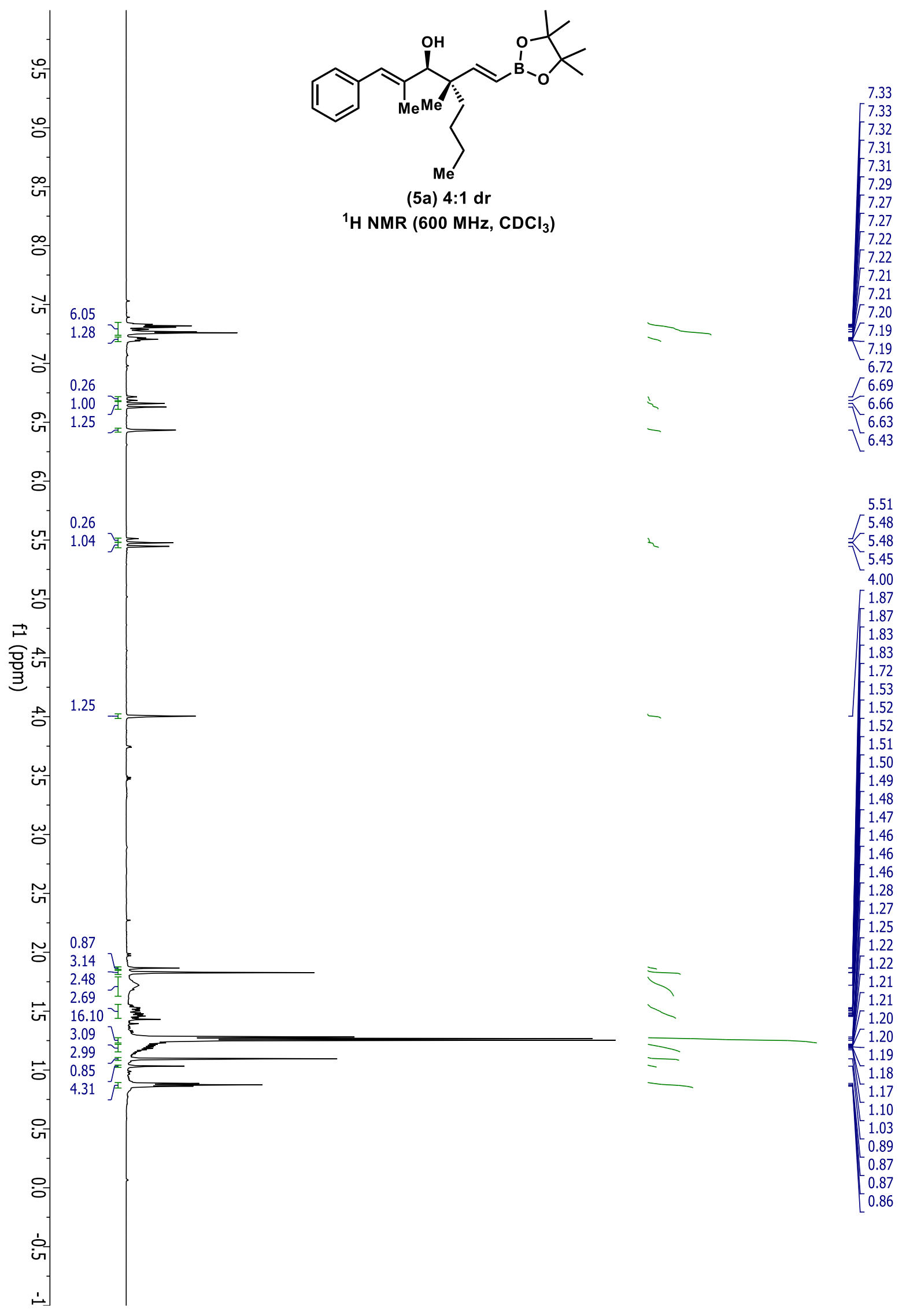




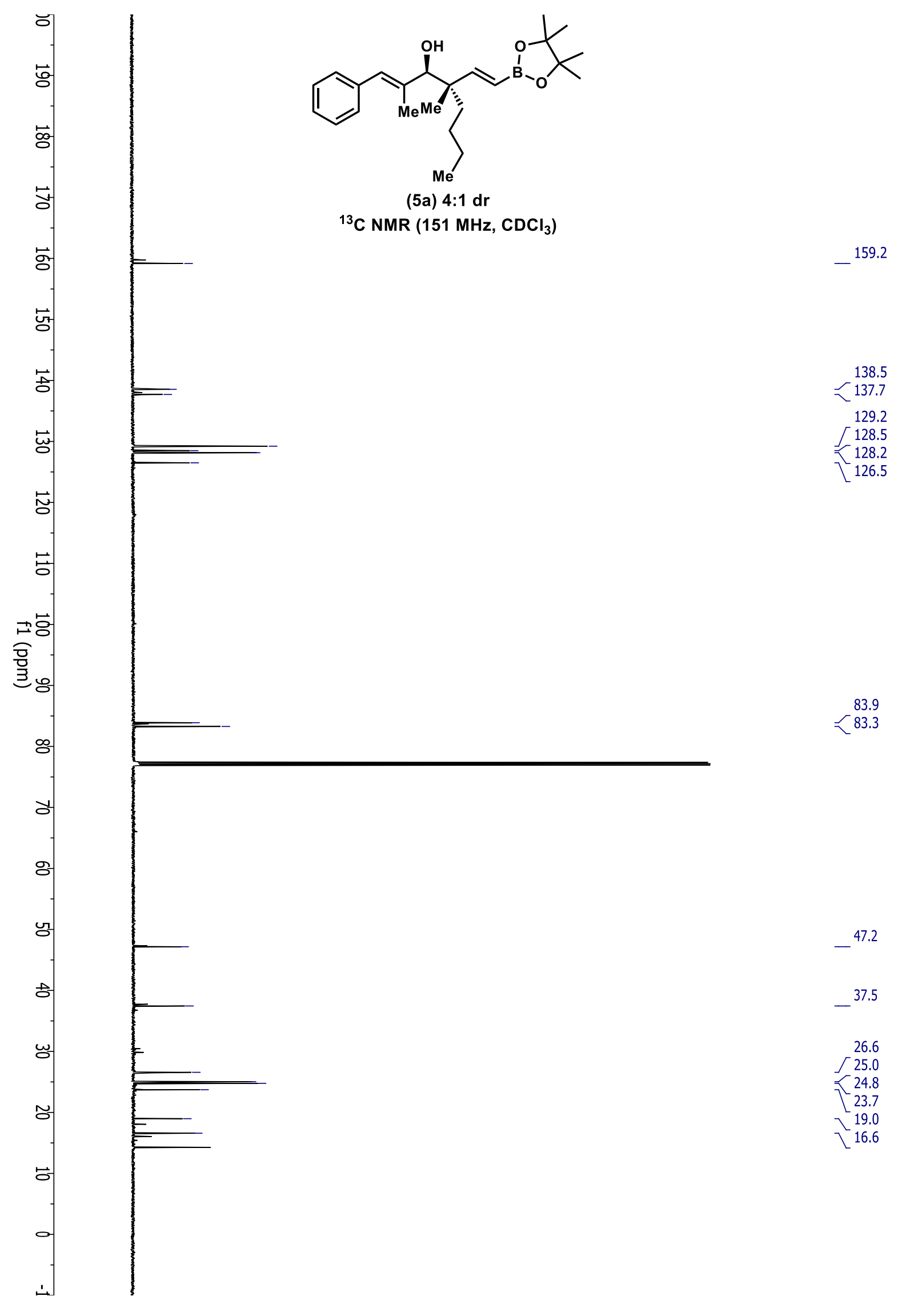




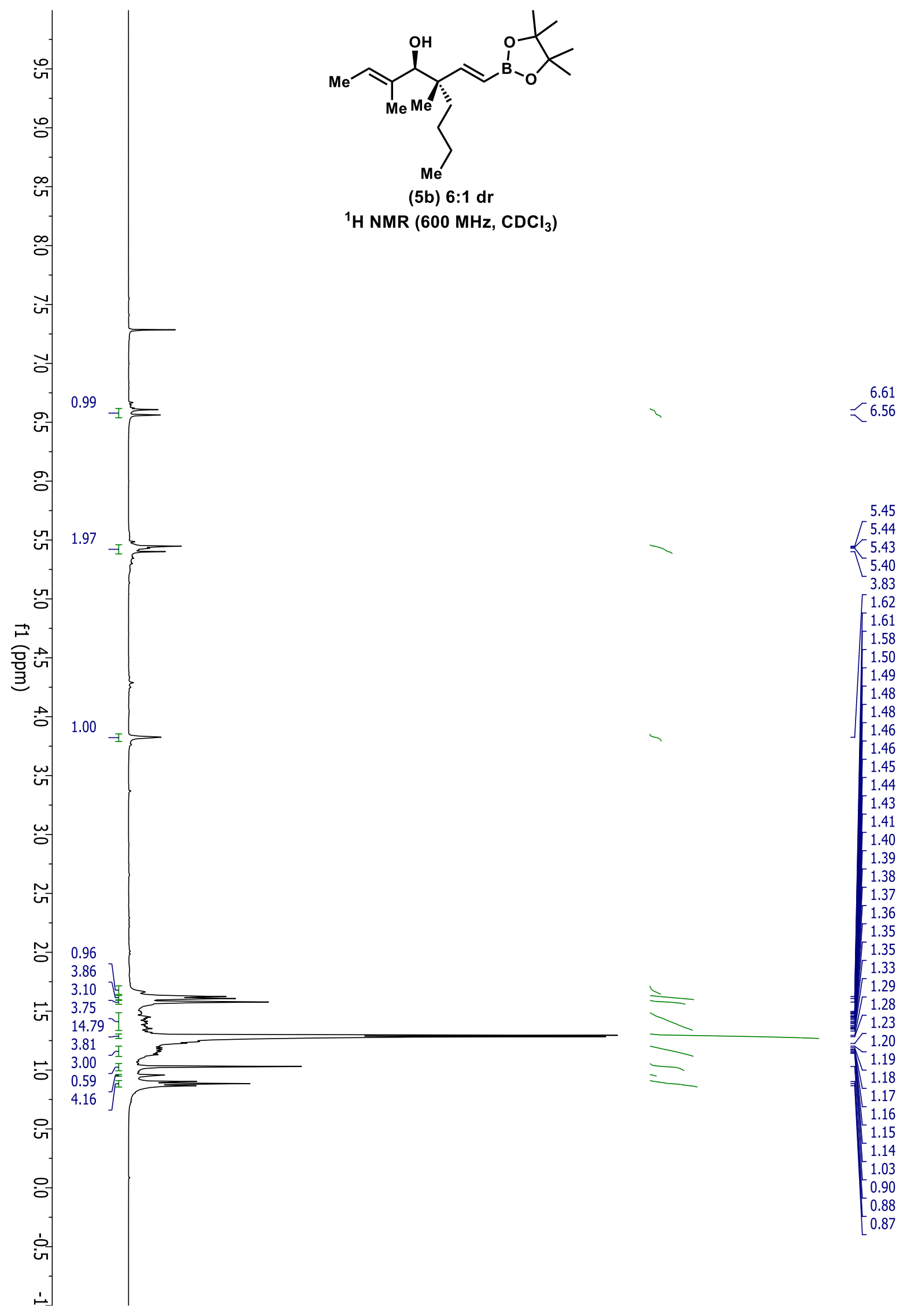




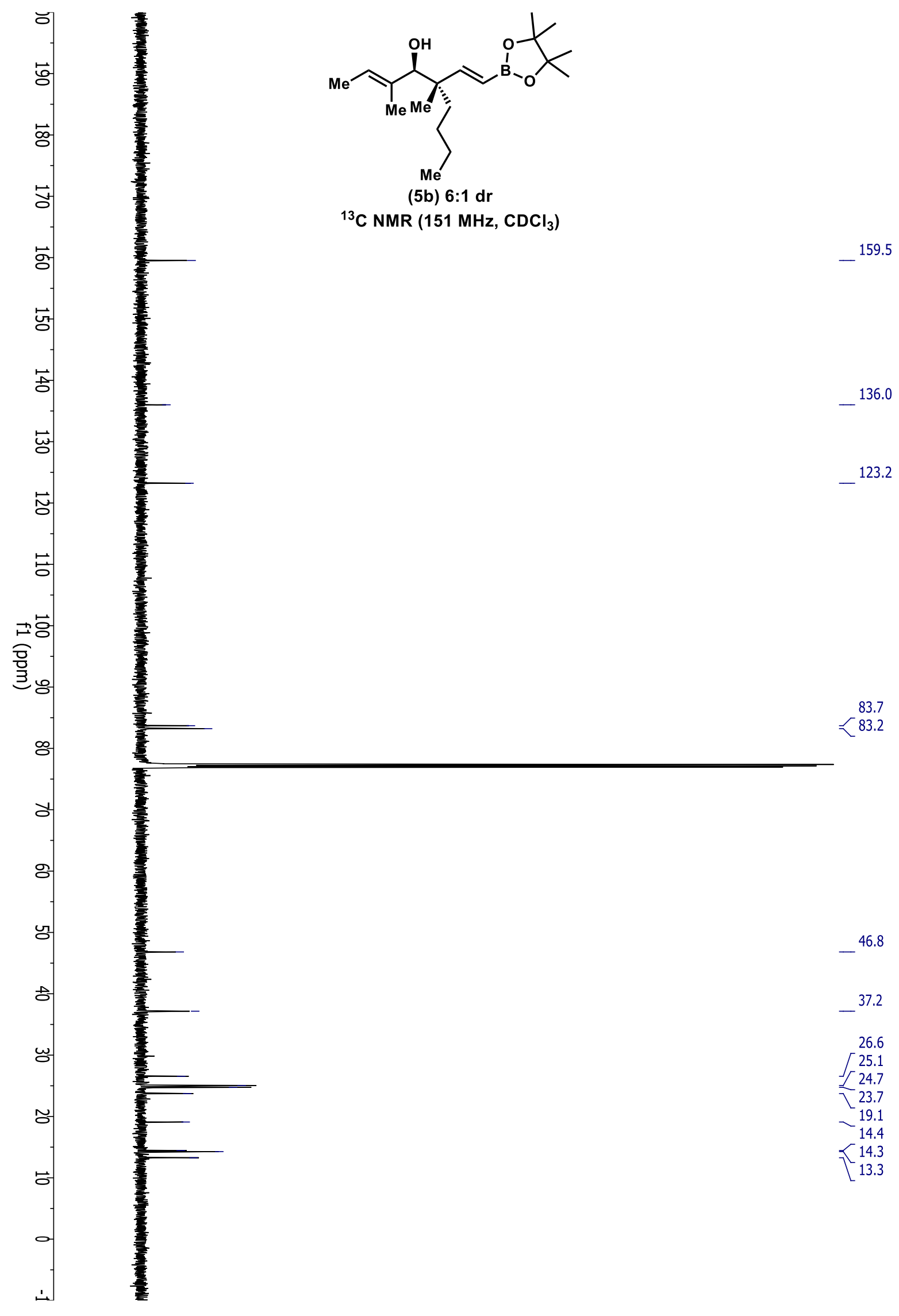




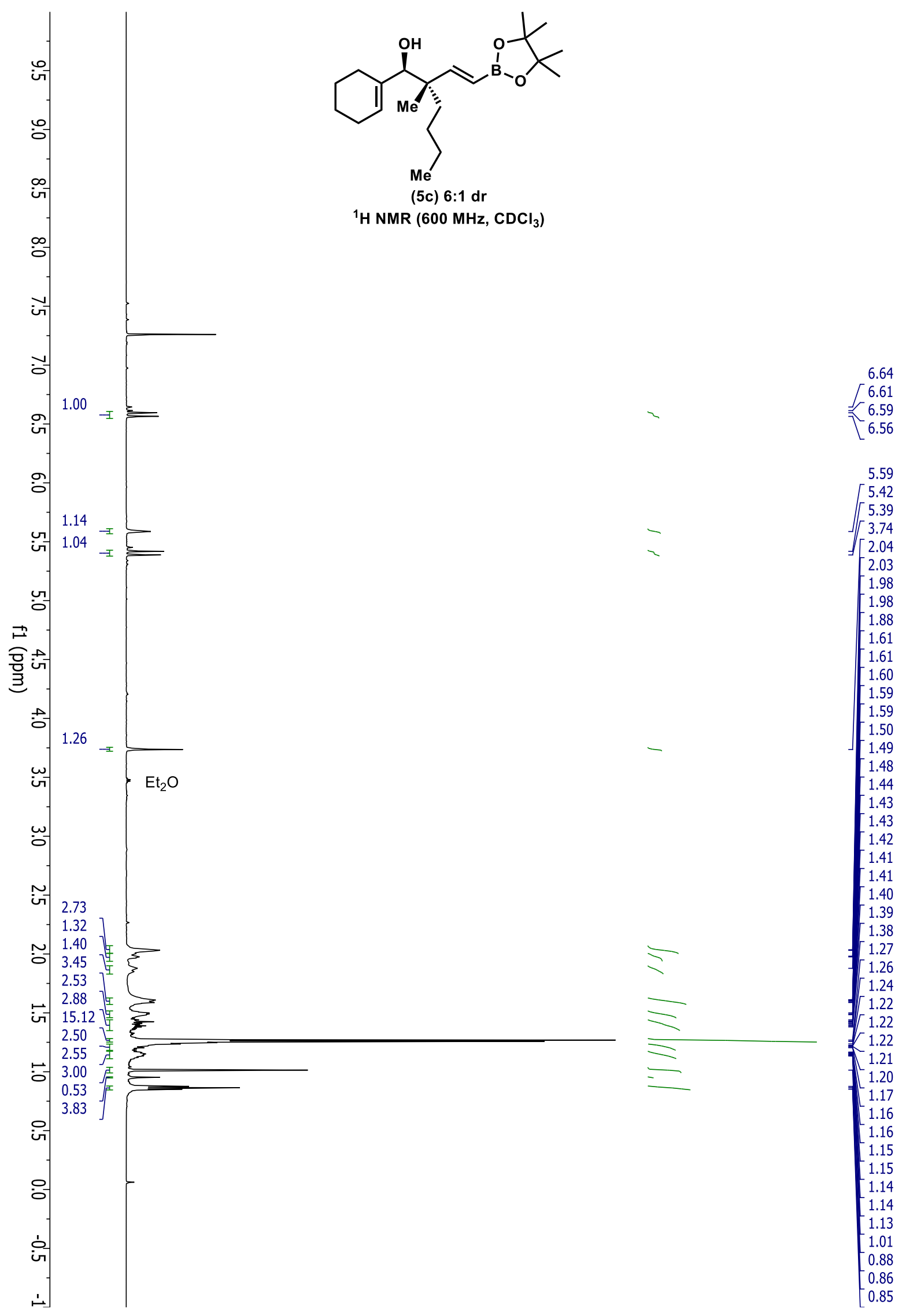




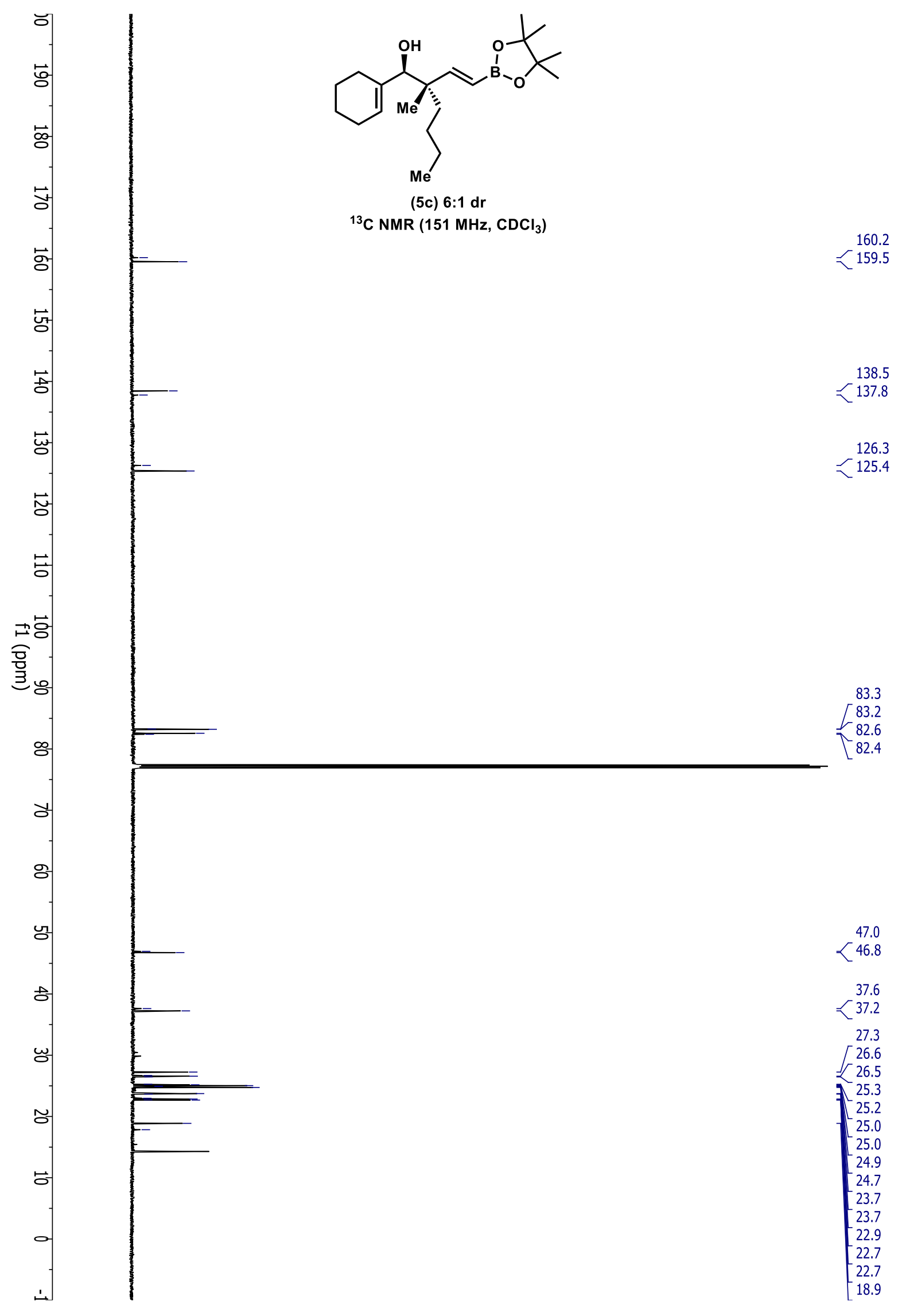



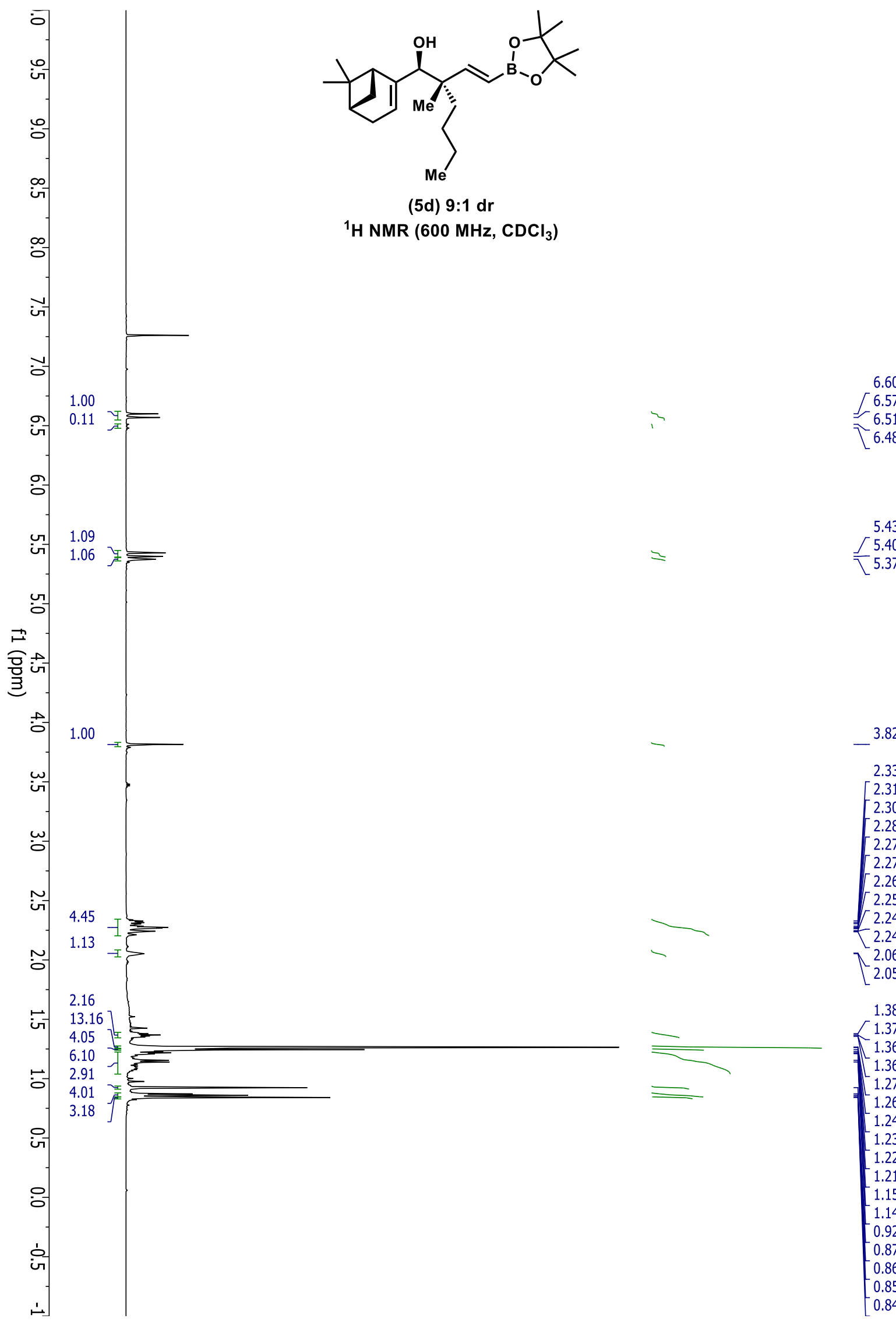

6.60
$\Gamma-57$
-6.51
-6.48

5.43

โ5.37

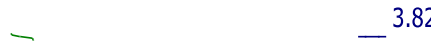

2.33

2.31

2.30

2.28

2.27

2.27

2.26

2.25

$=\quad+\quad \begin{aligned} & 2.24 \\ & 2.24 \\ & 2.06\end{aligned}$

$\checkmark$

2.06
2.05

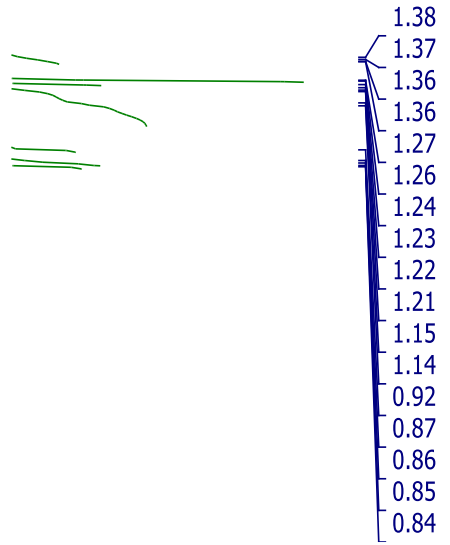




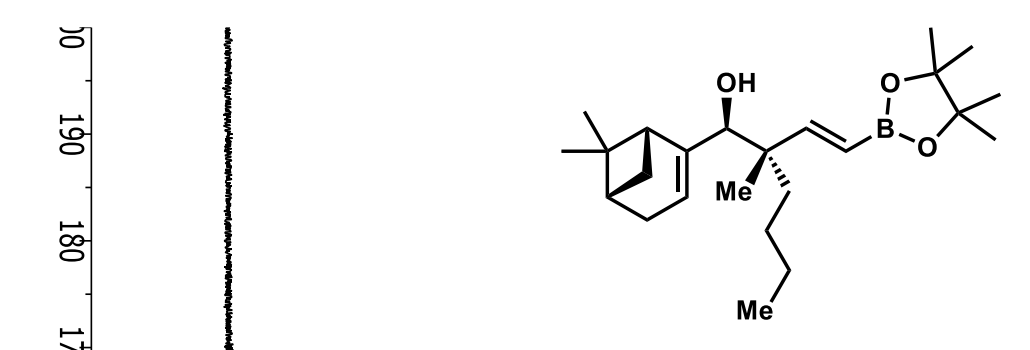

(5d) 9:1 dr

${ }^{13} \mathrm{C}$ NMR (151 MHz, $\mathrm{CDCl}_{3}$ )
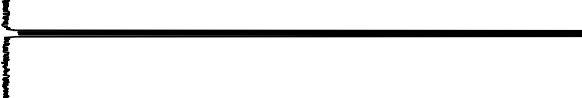


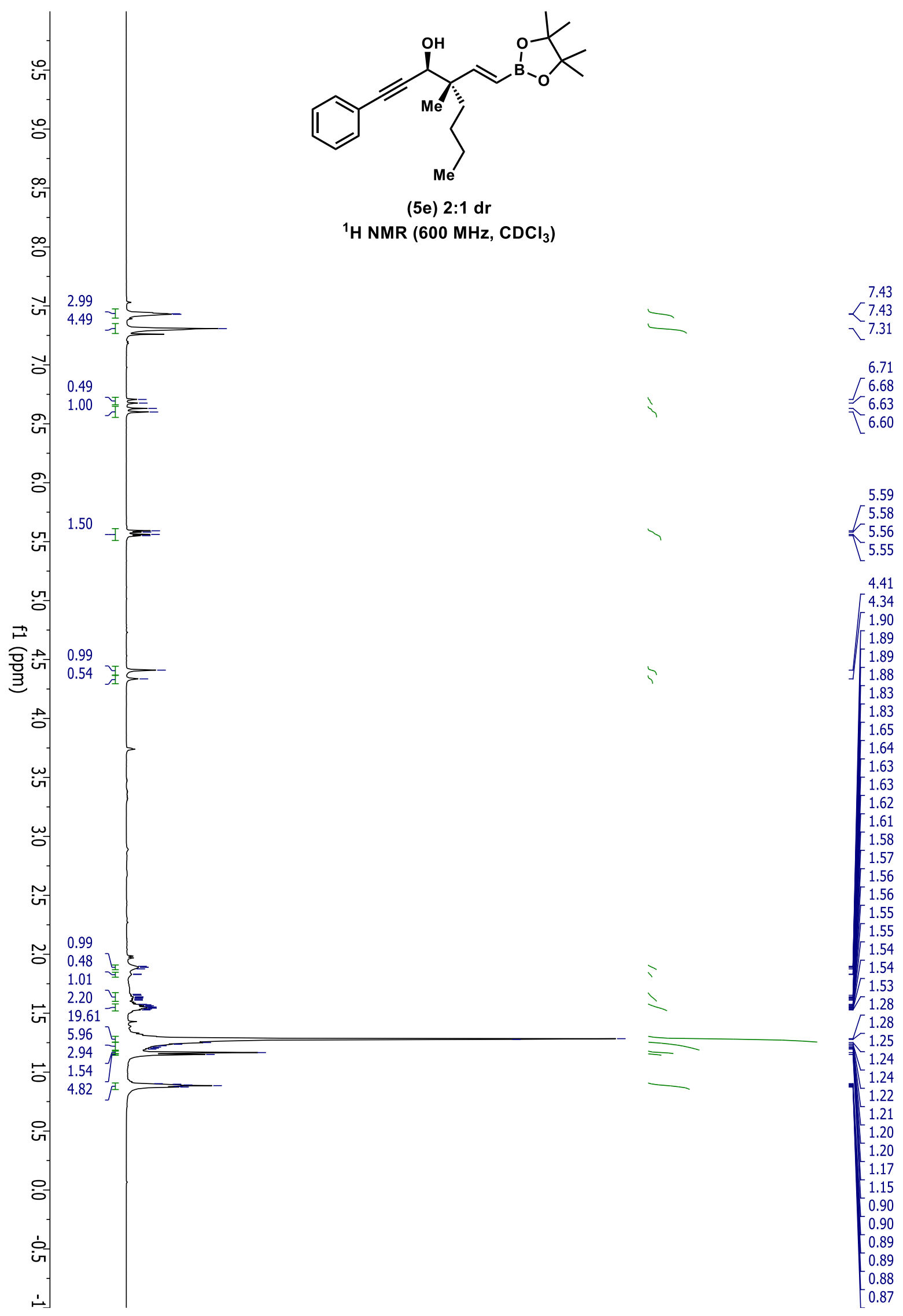




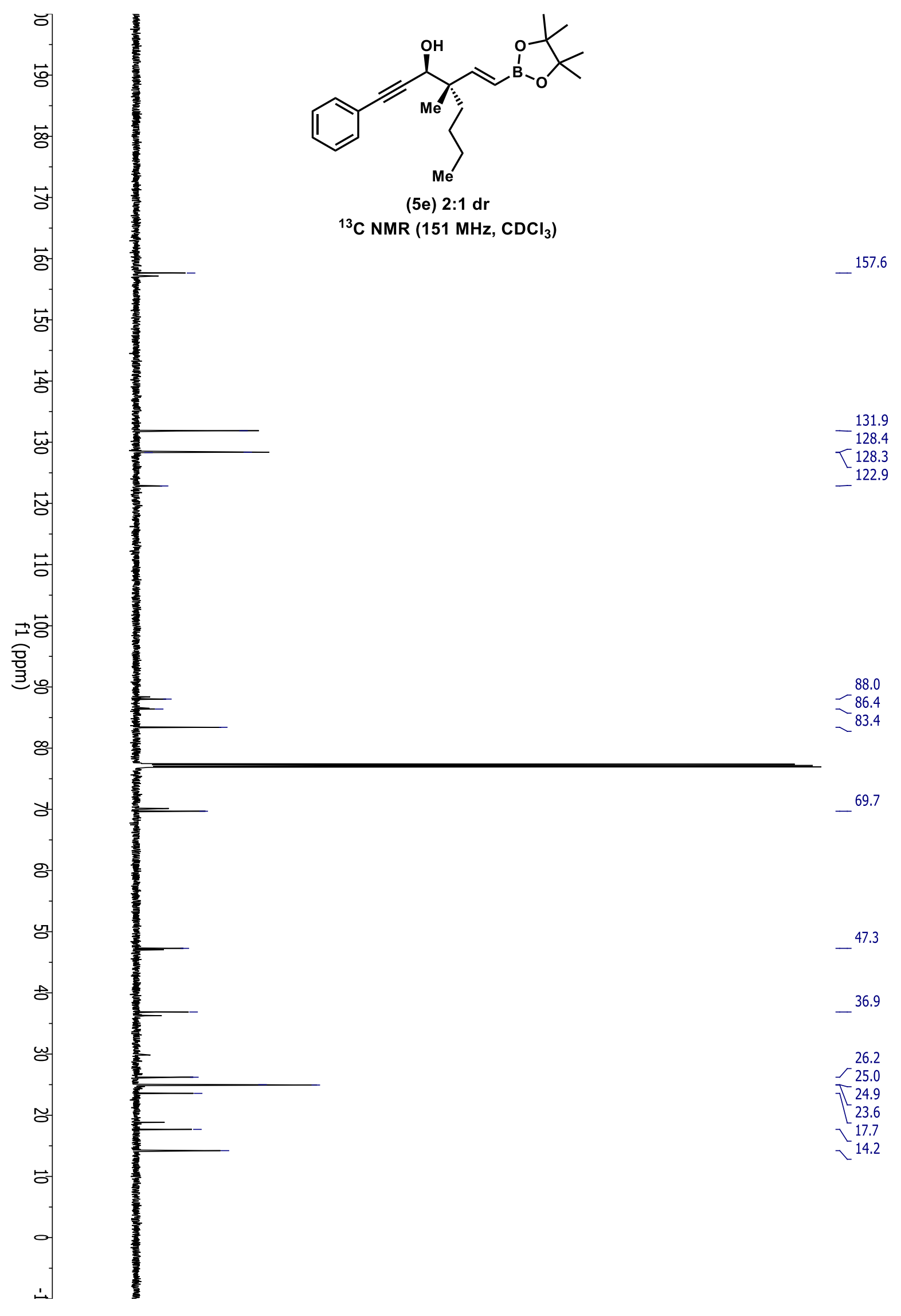




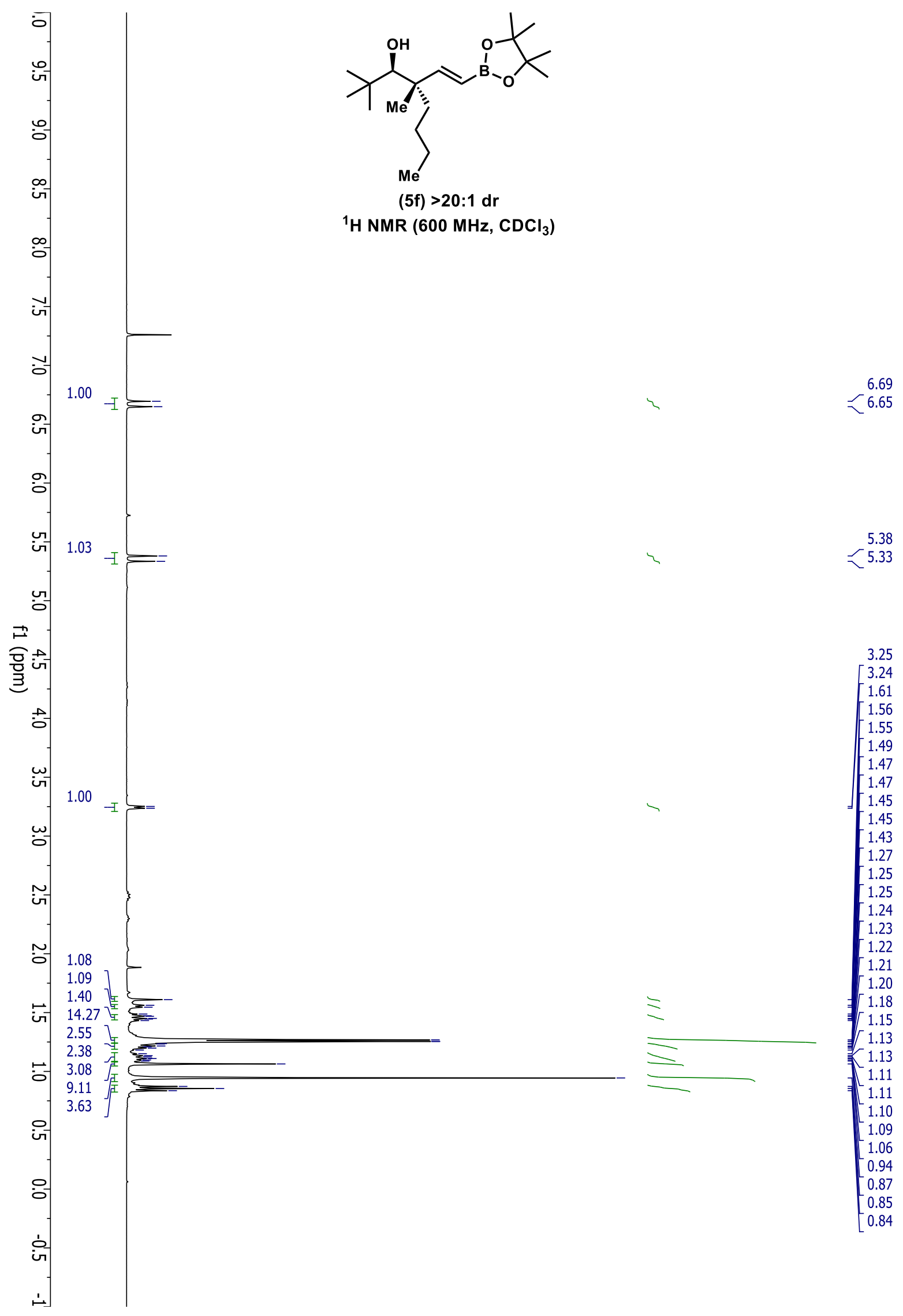




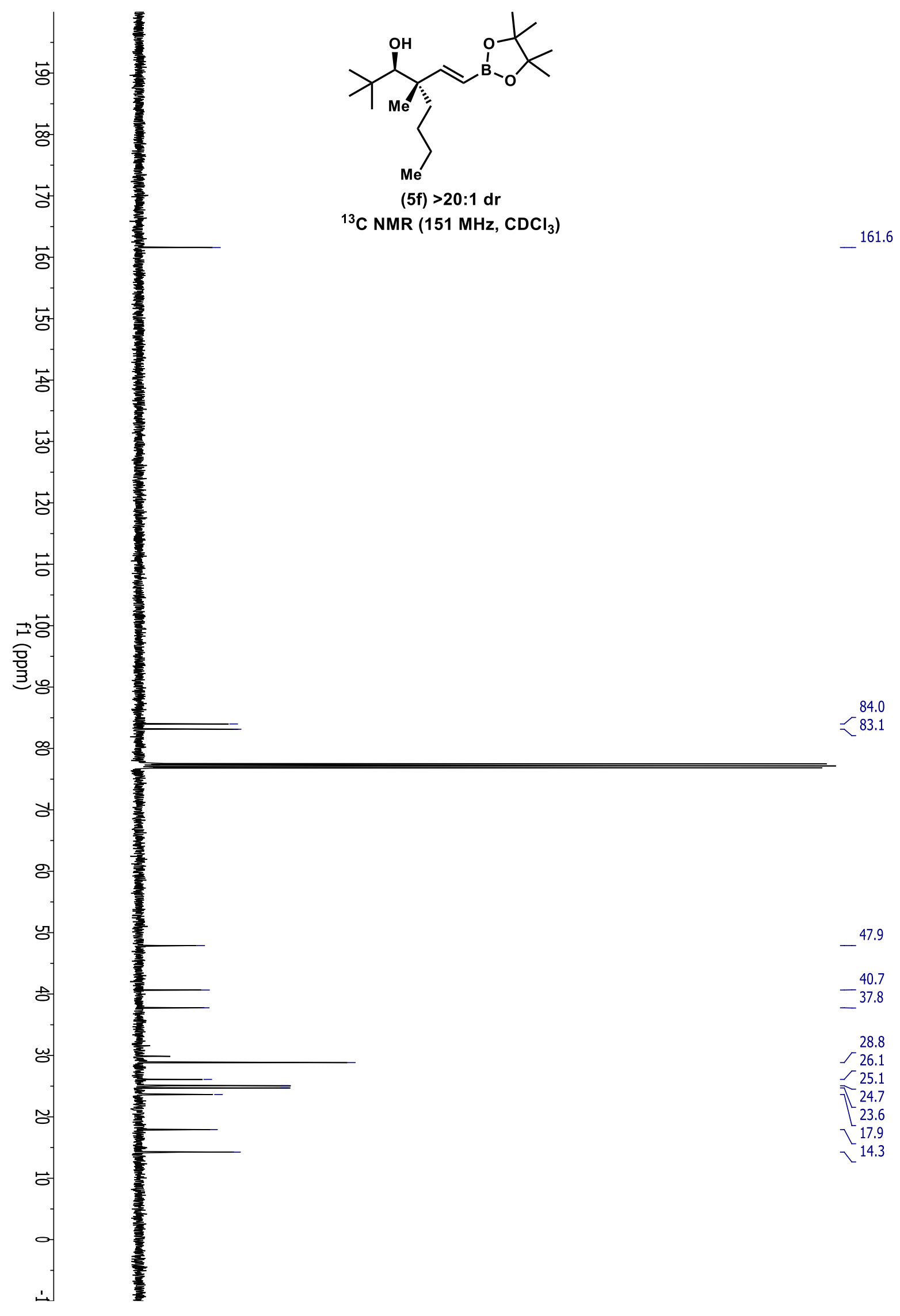




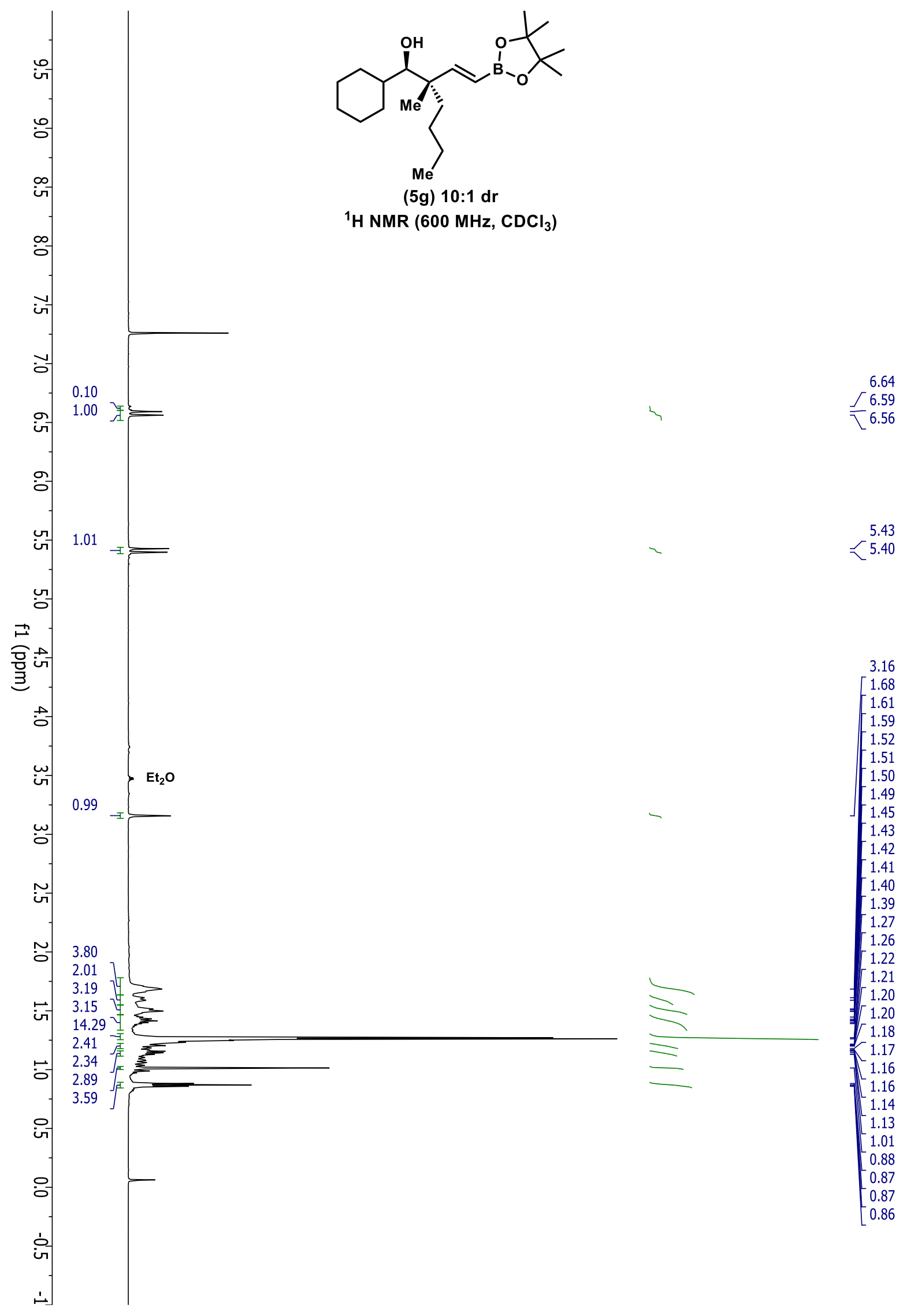




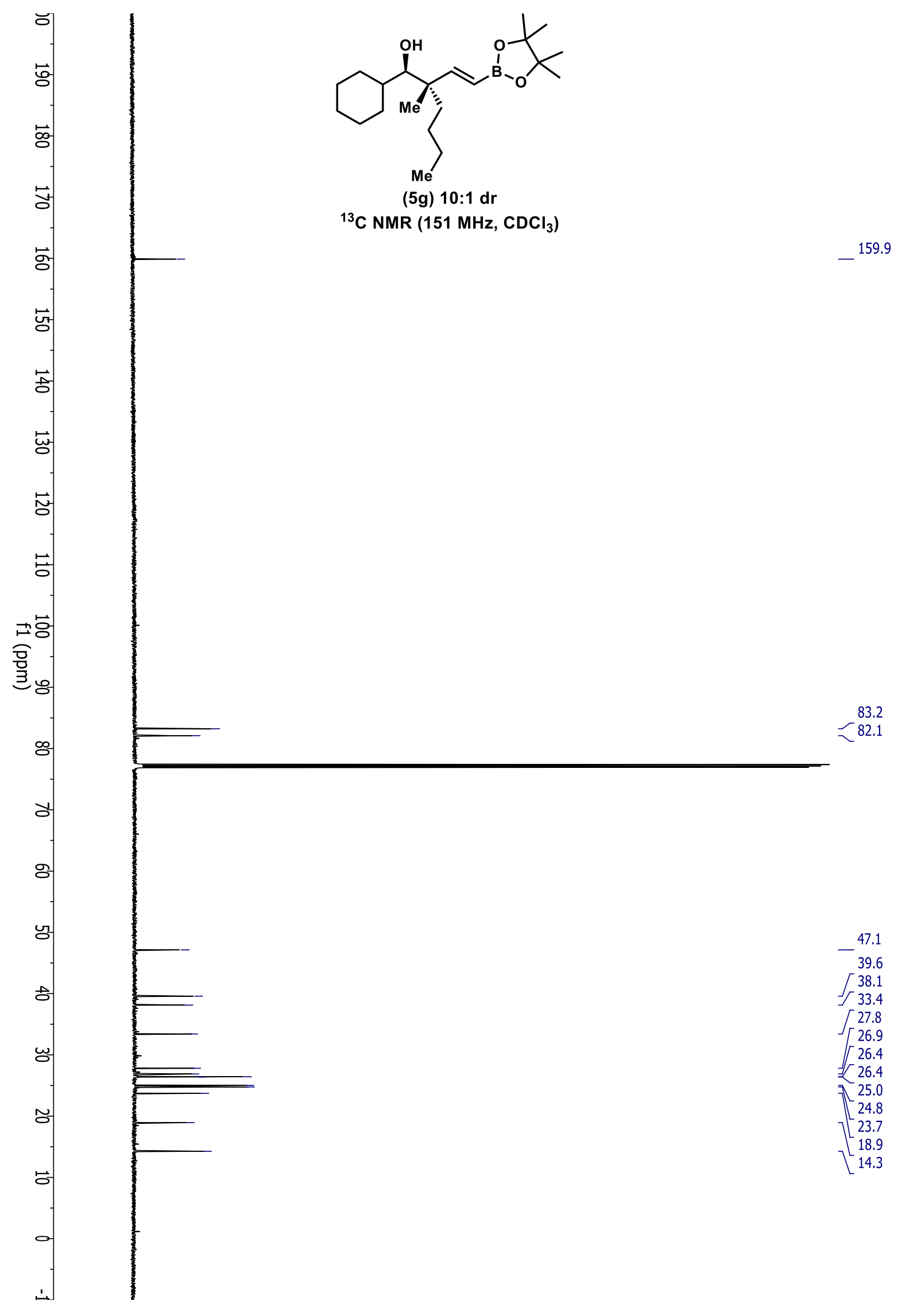




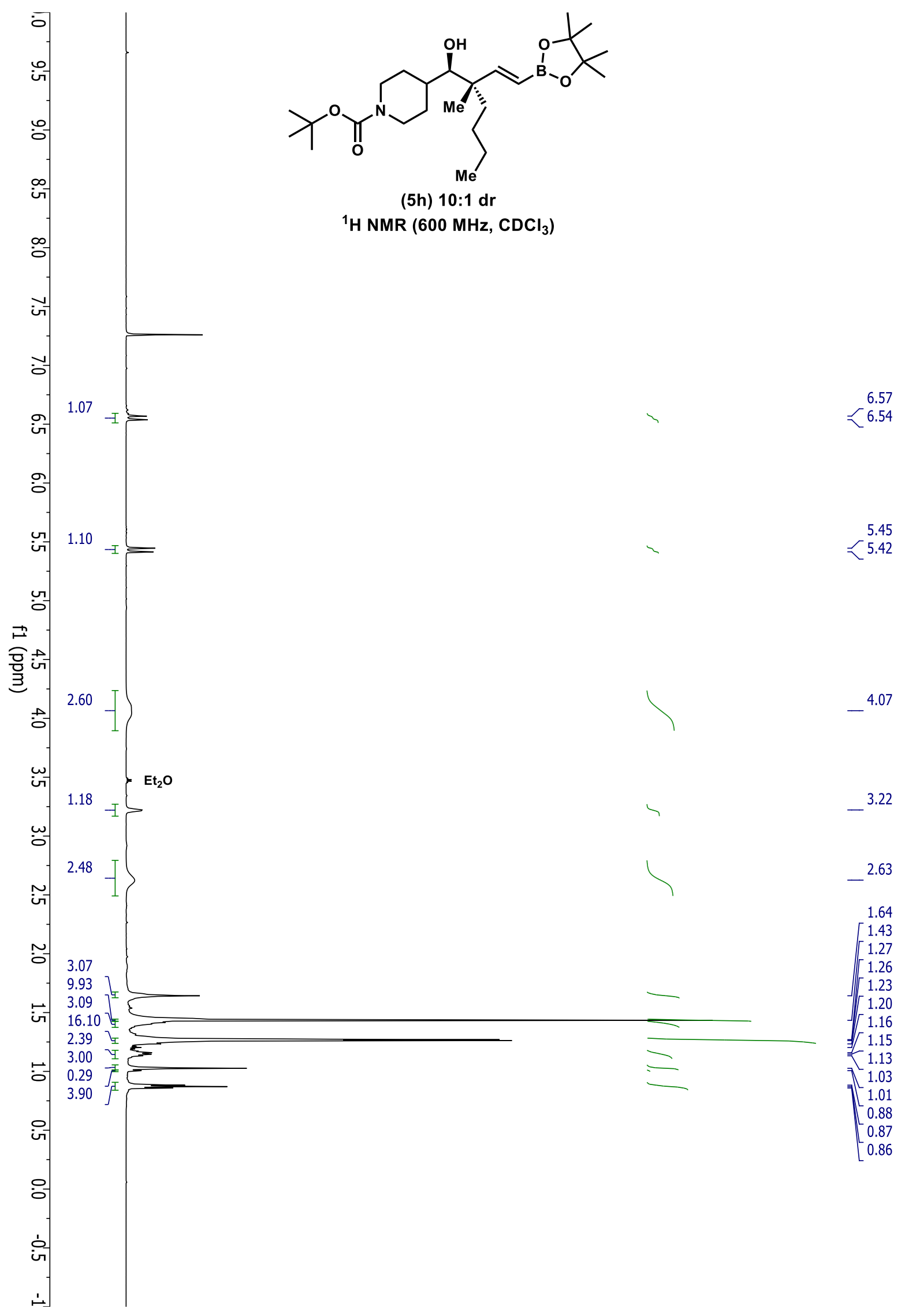




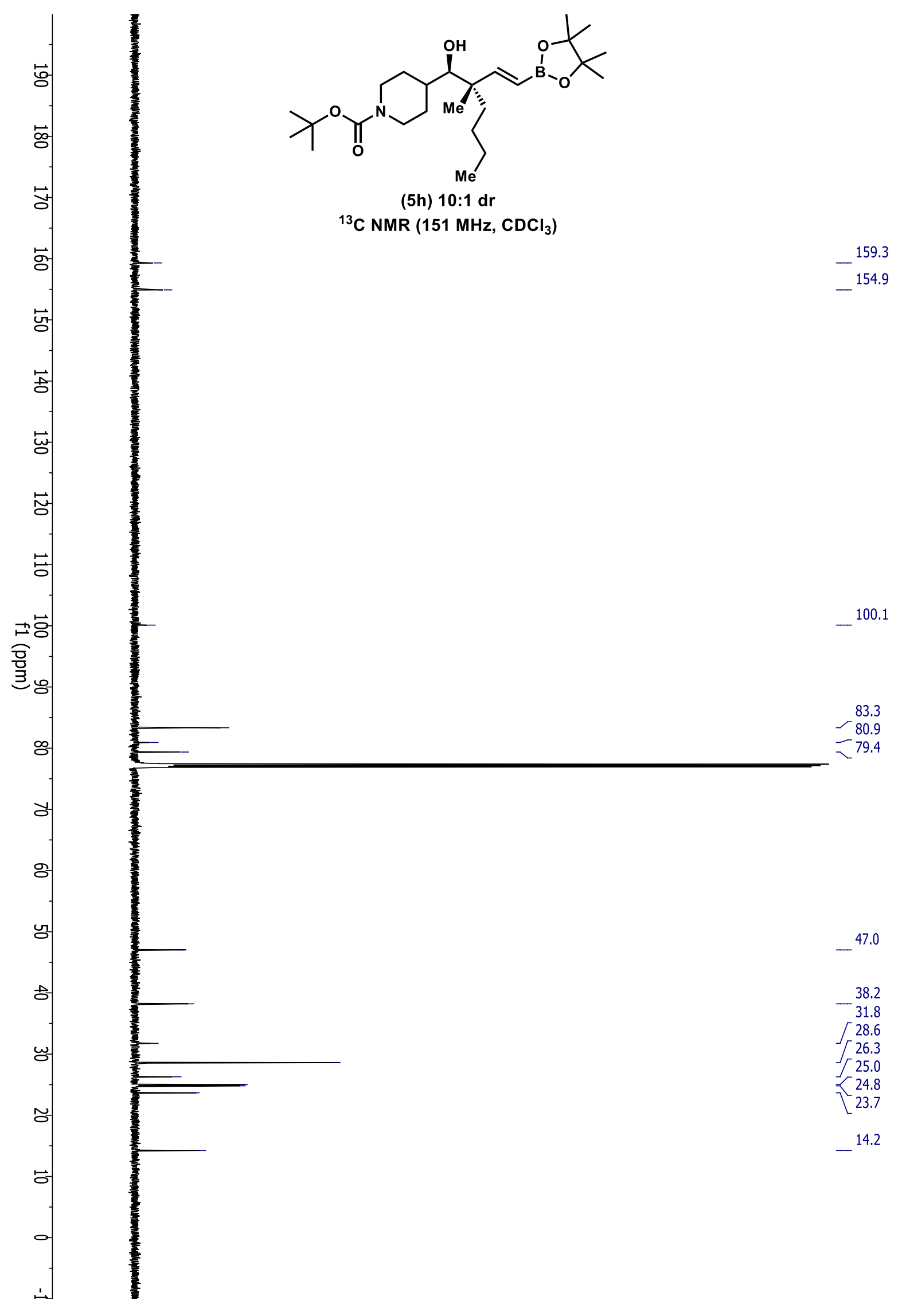



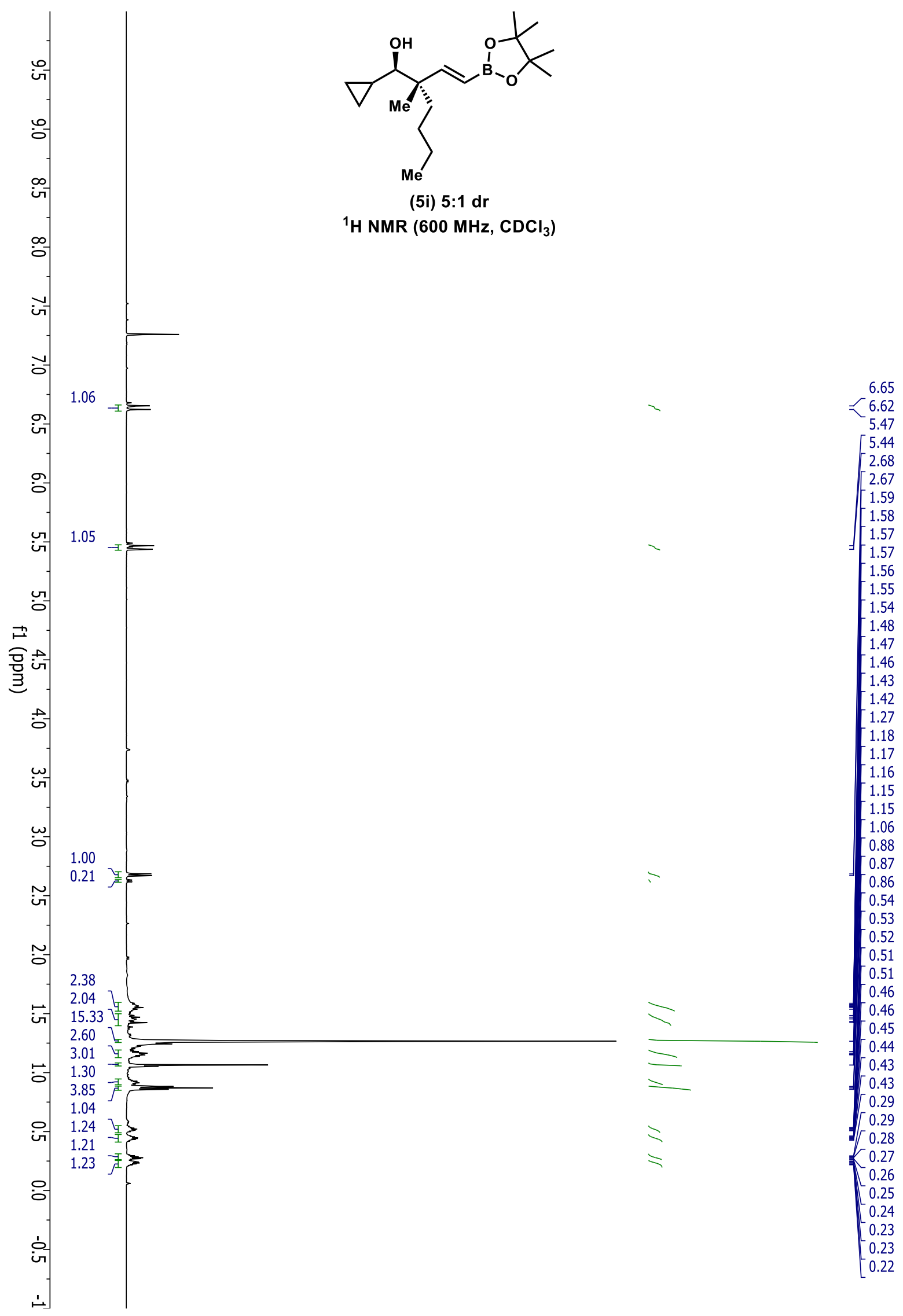


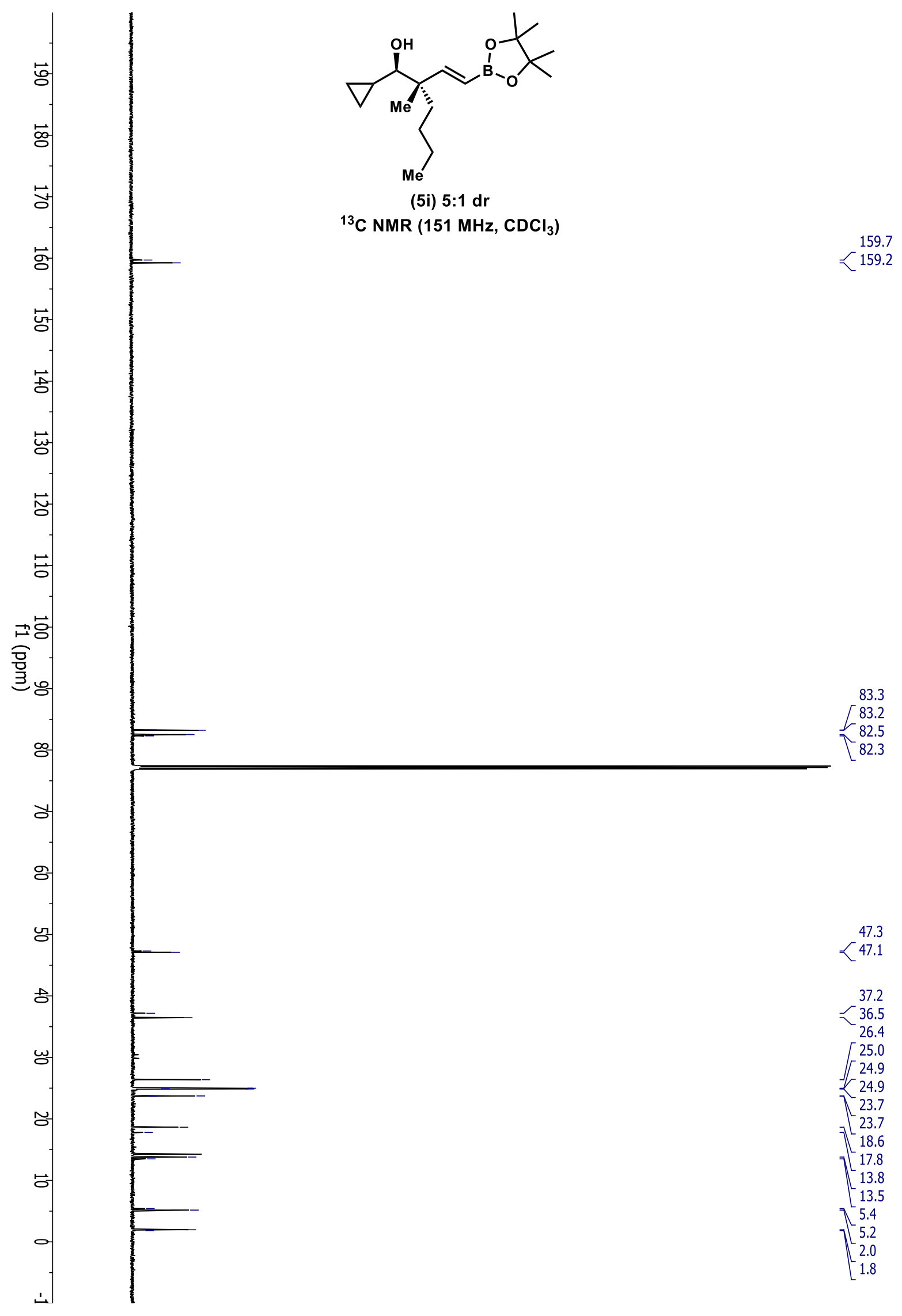



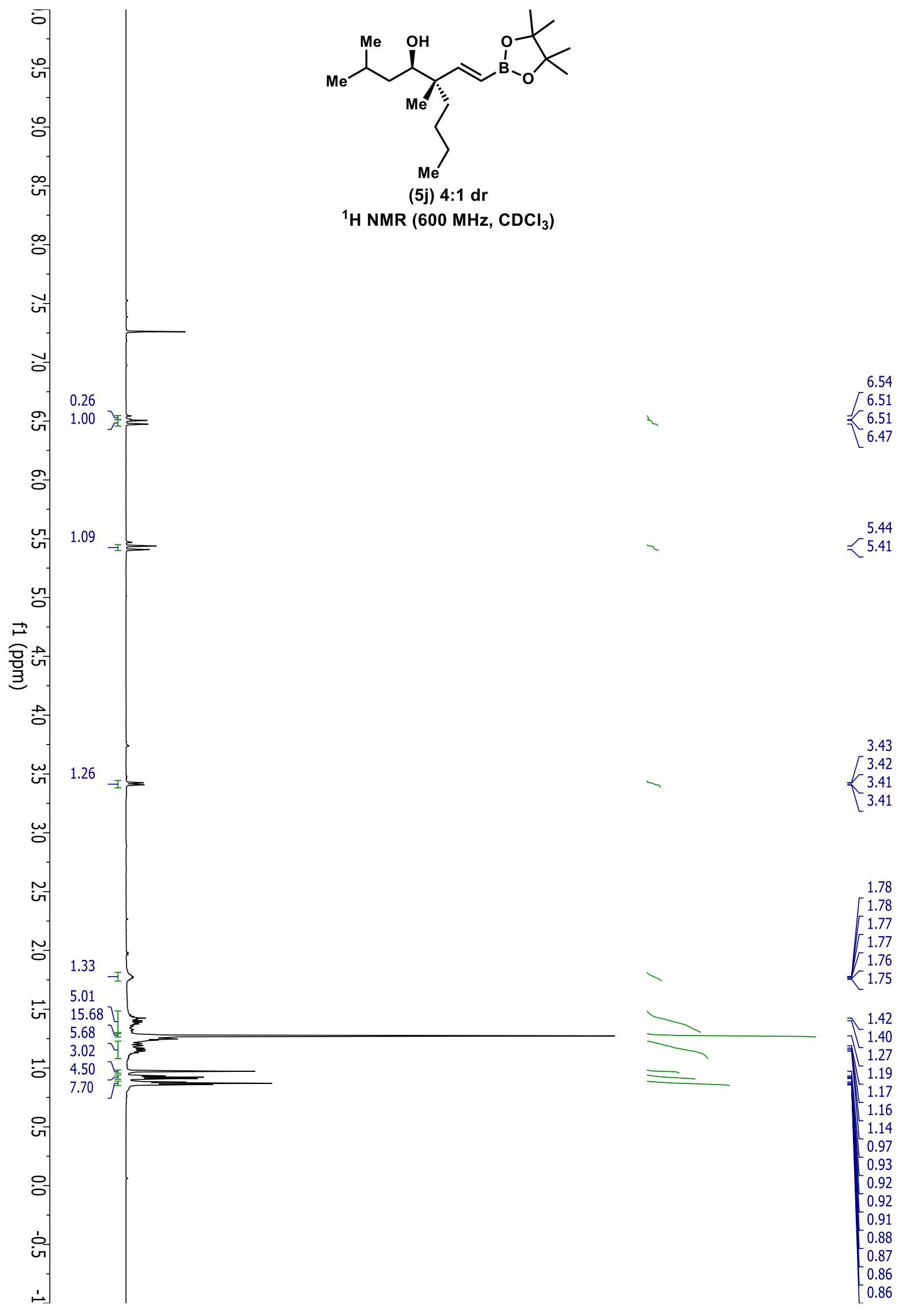


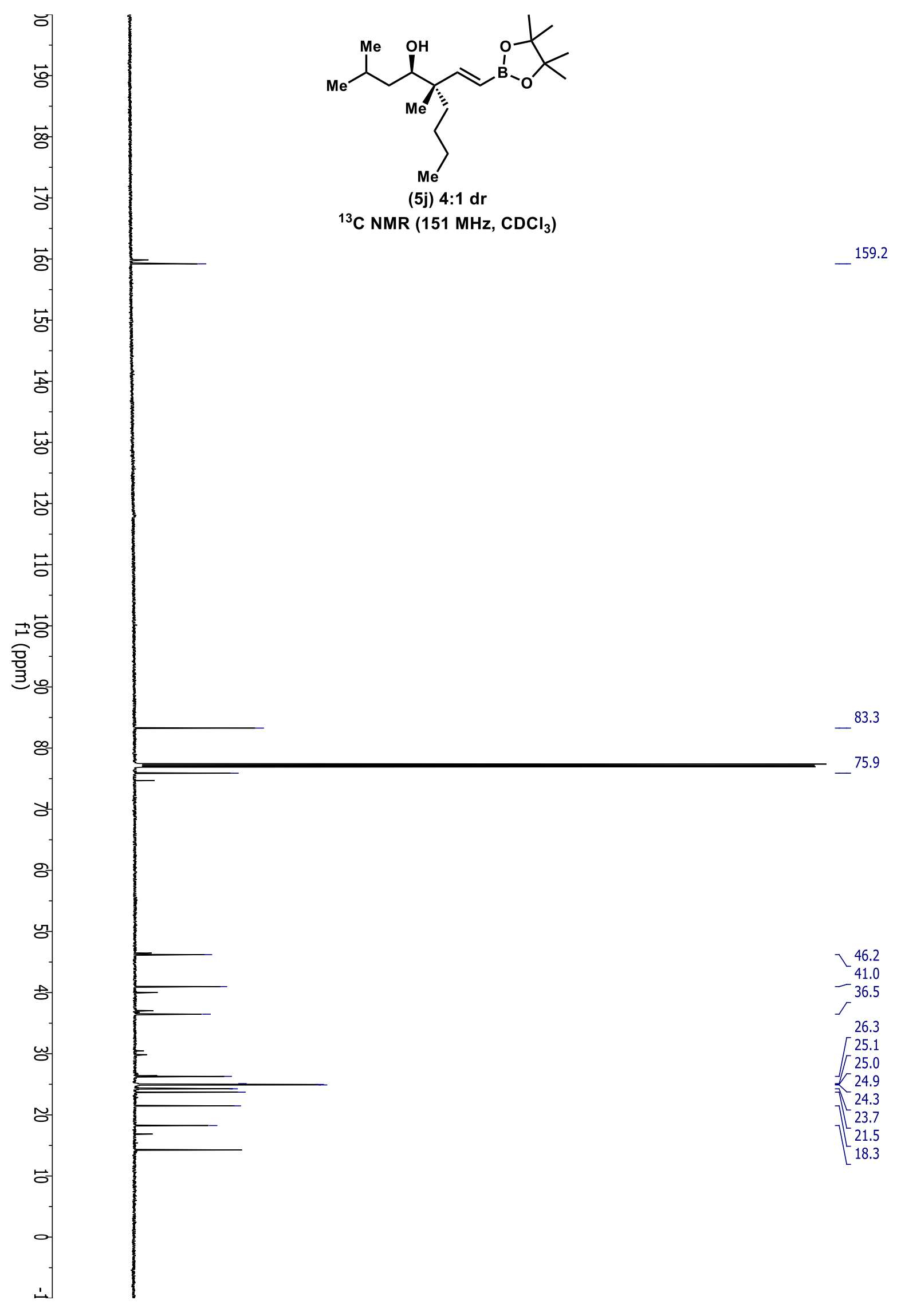




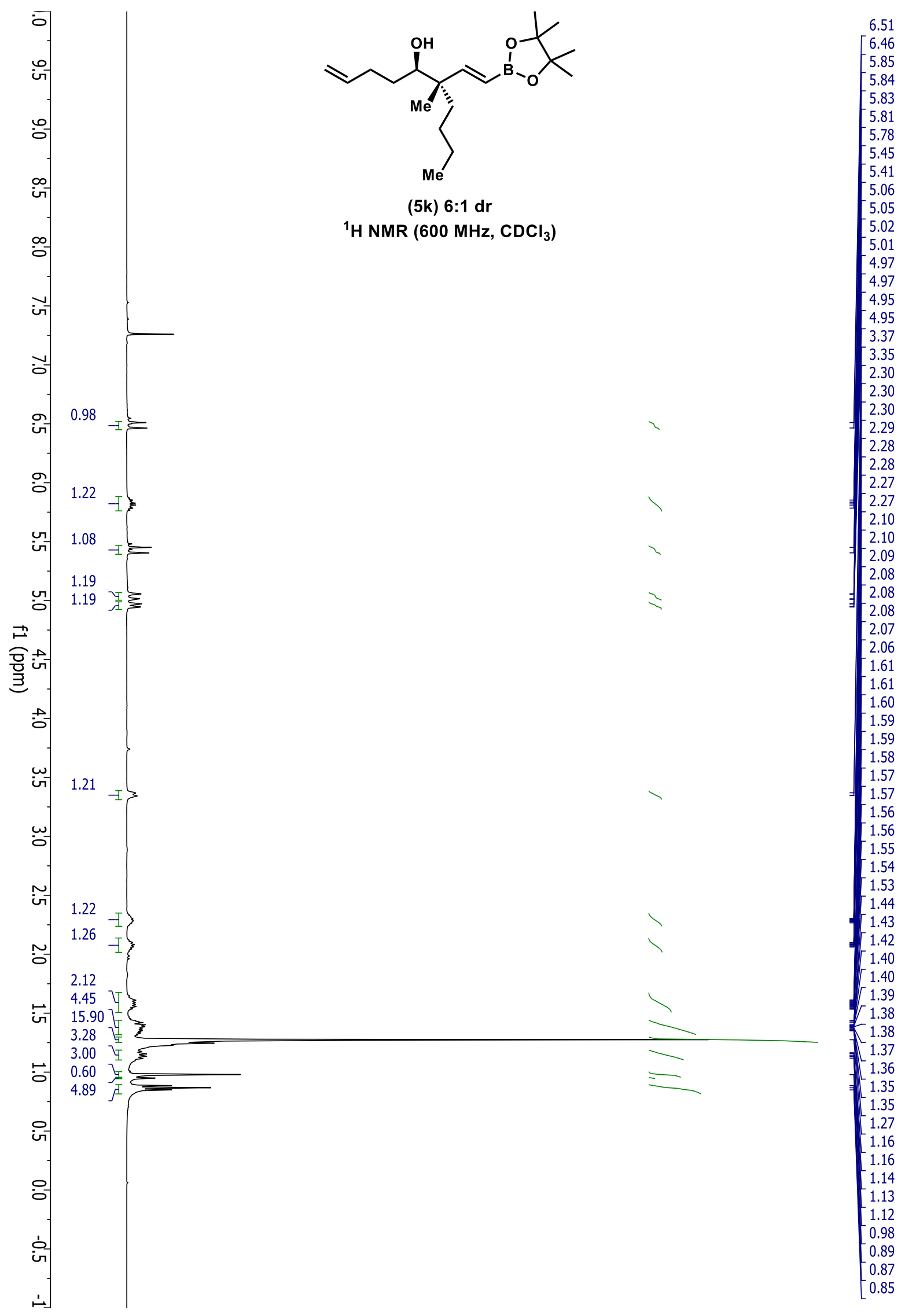




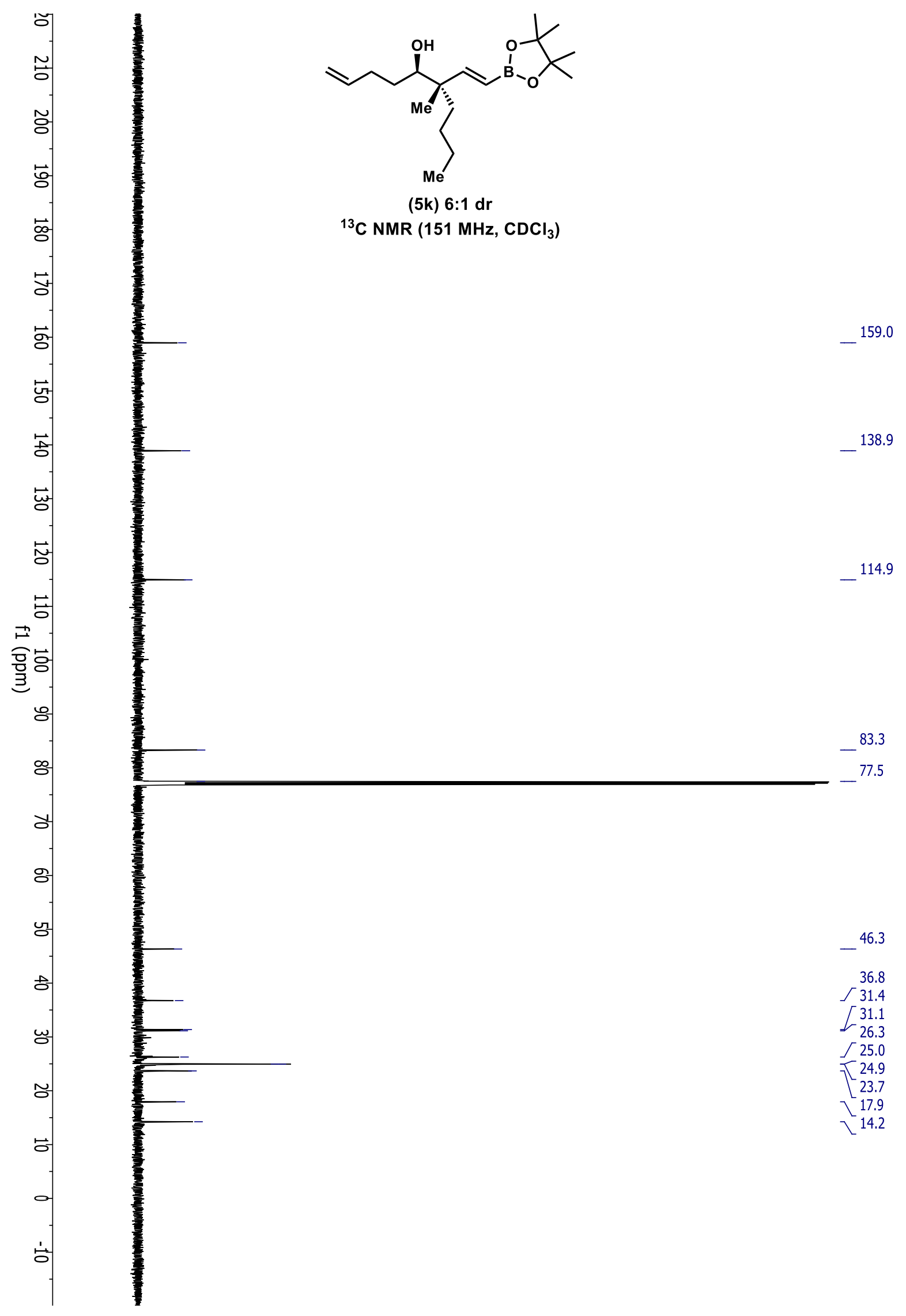




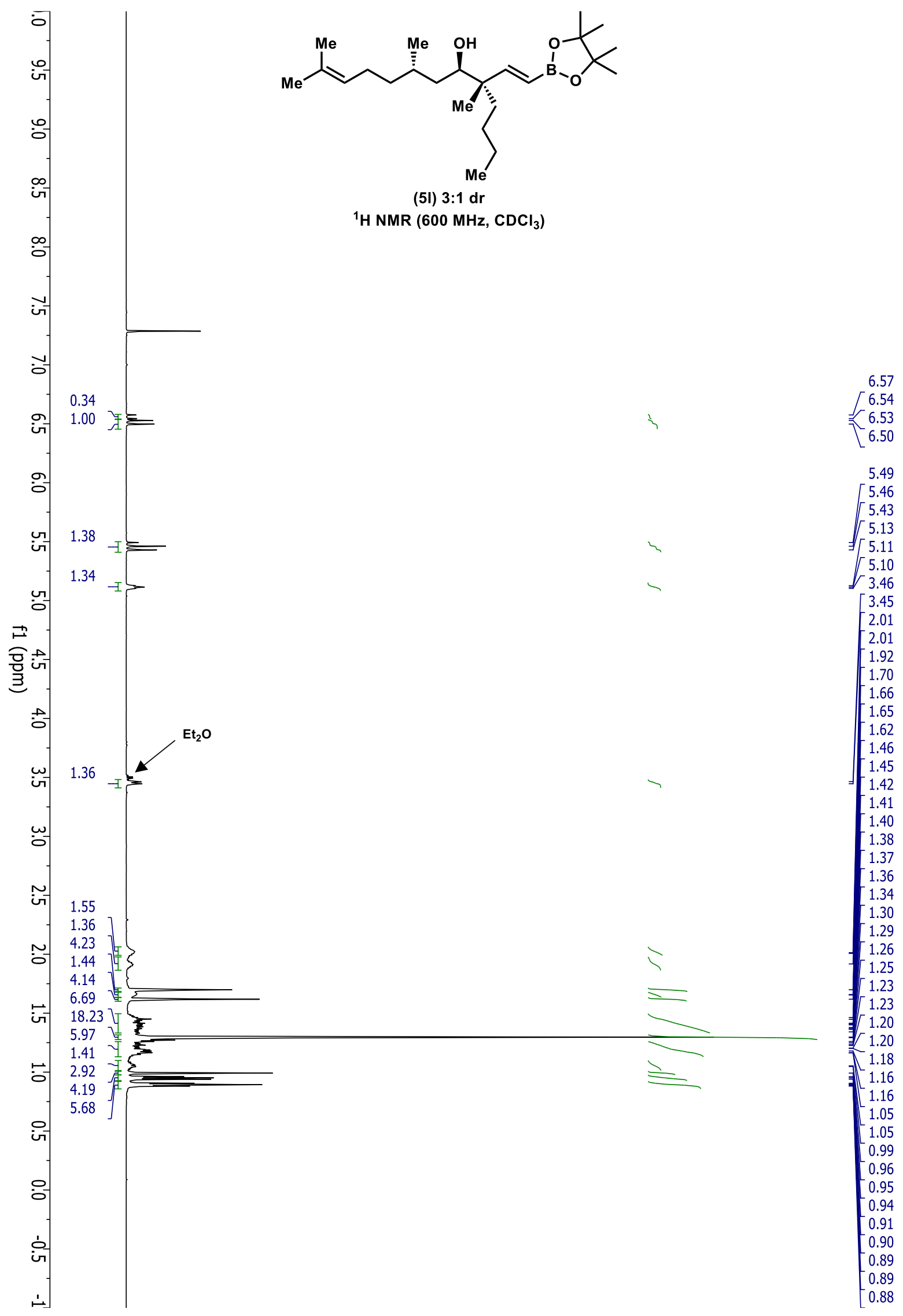




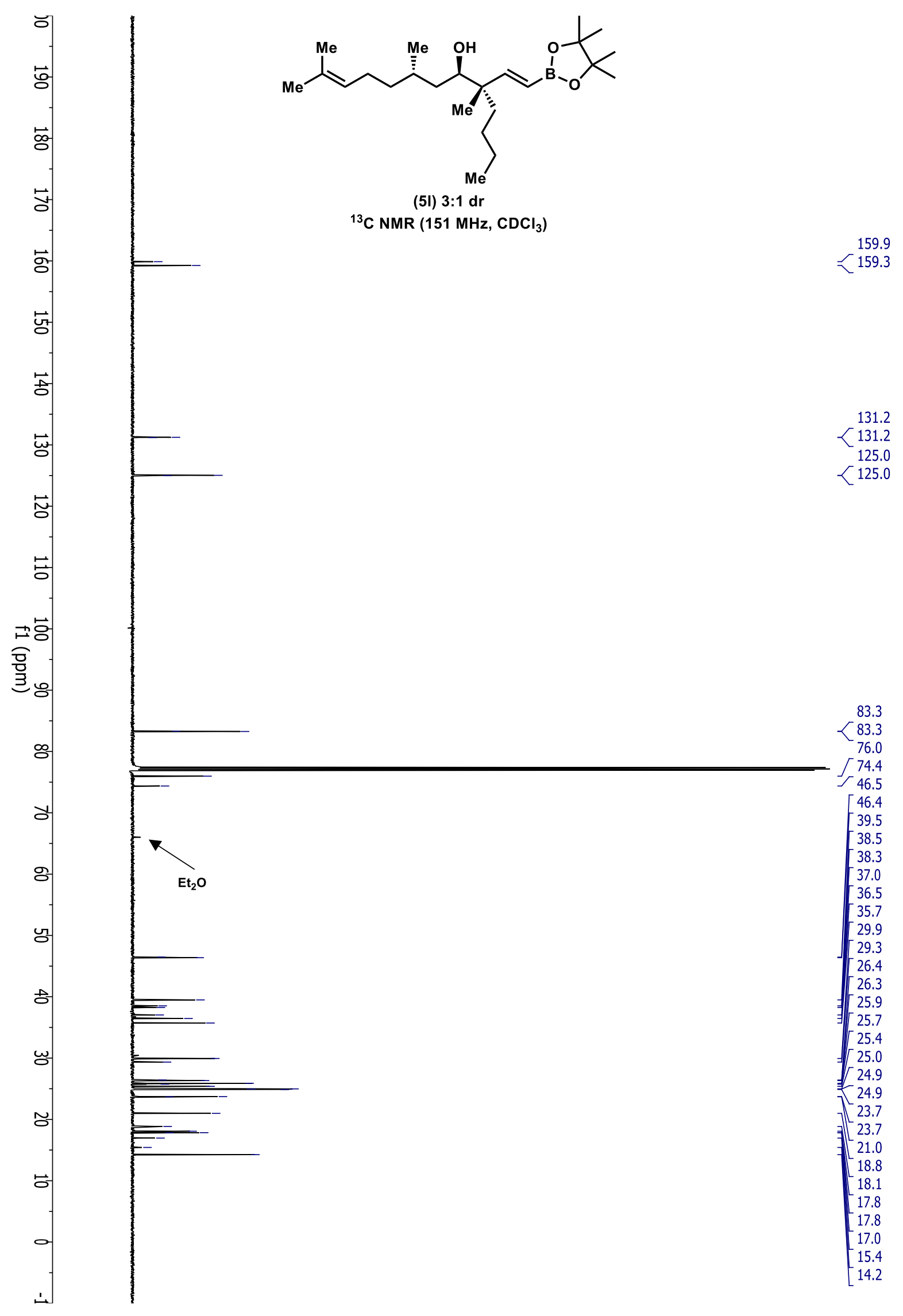




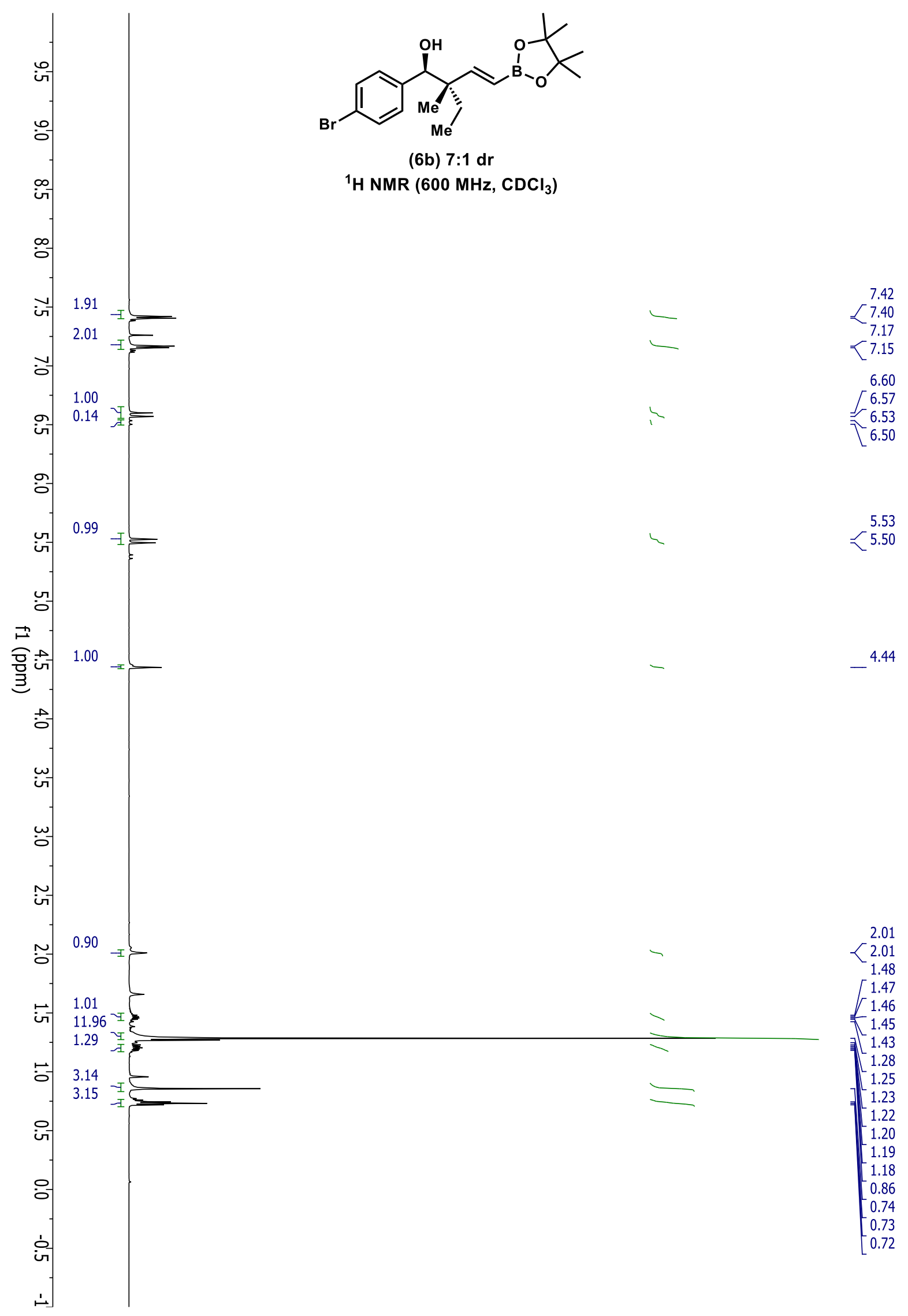




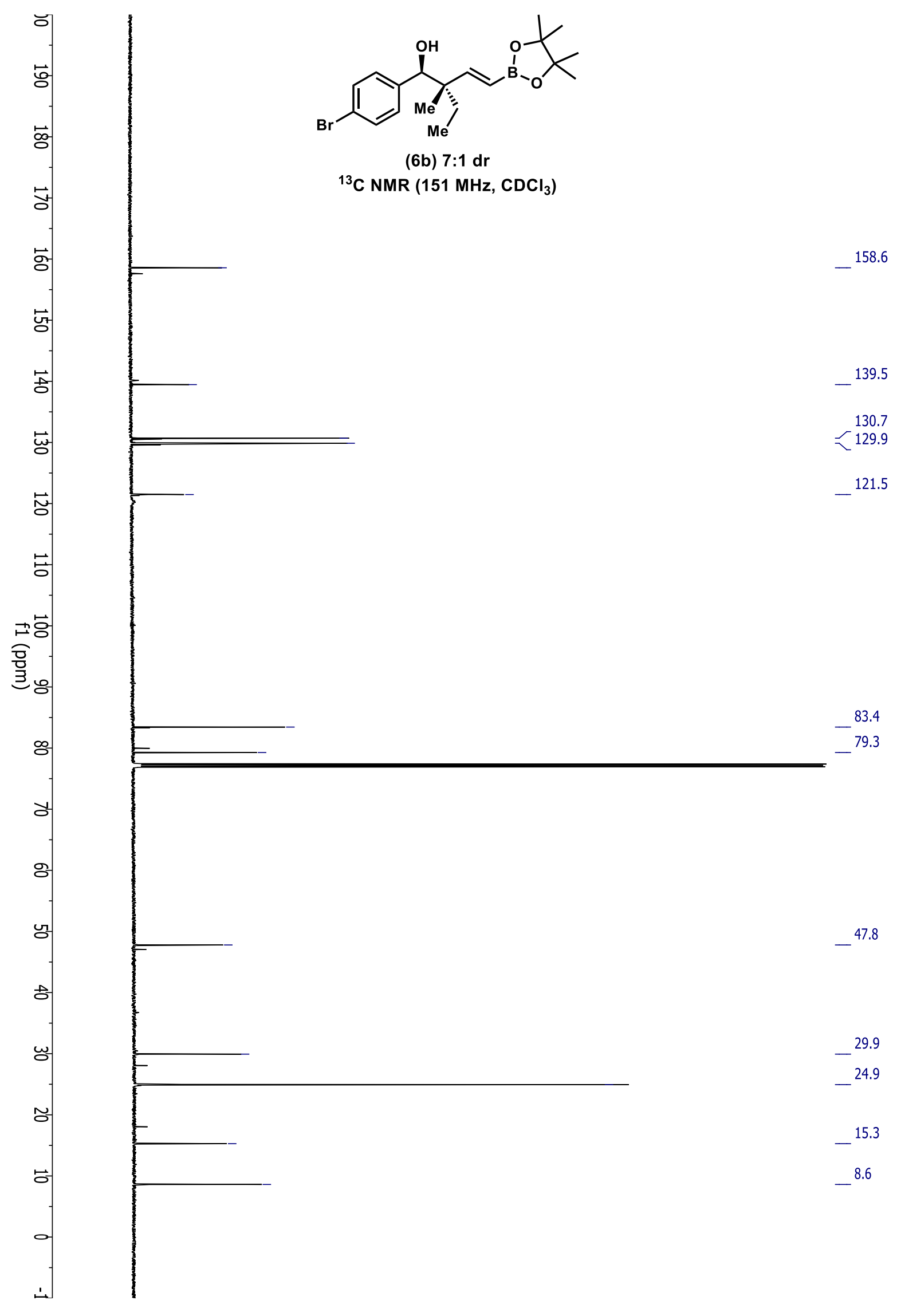




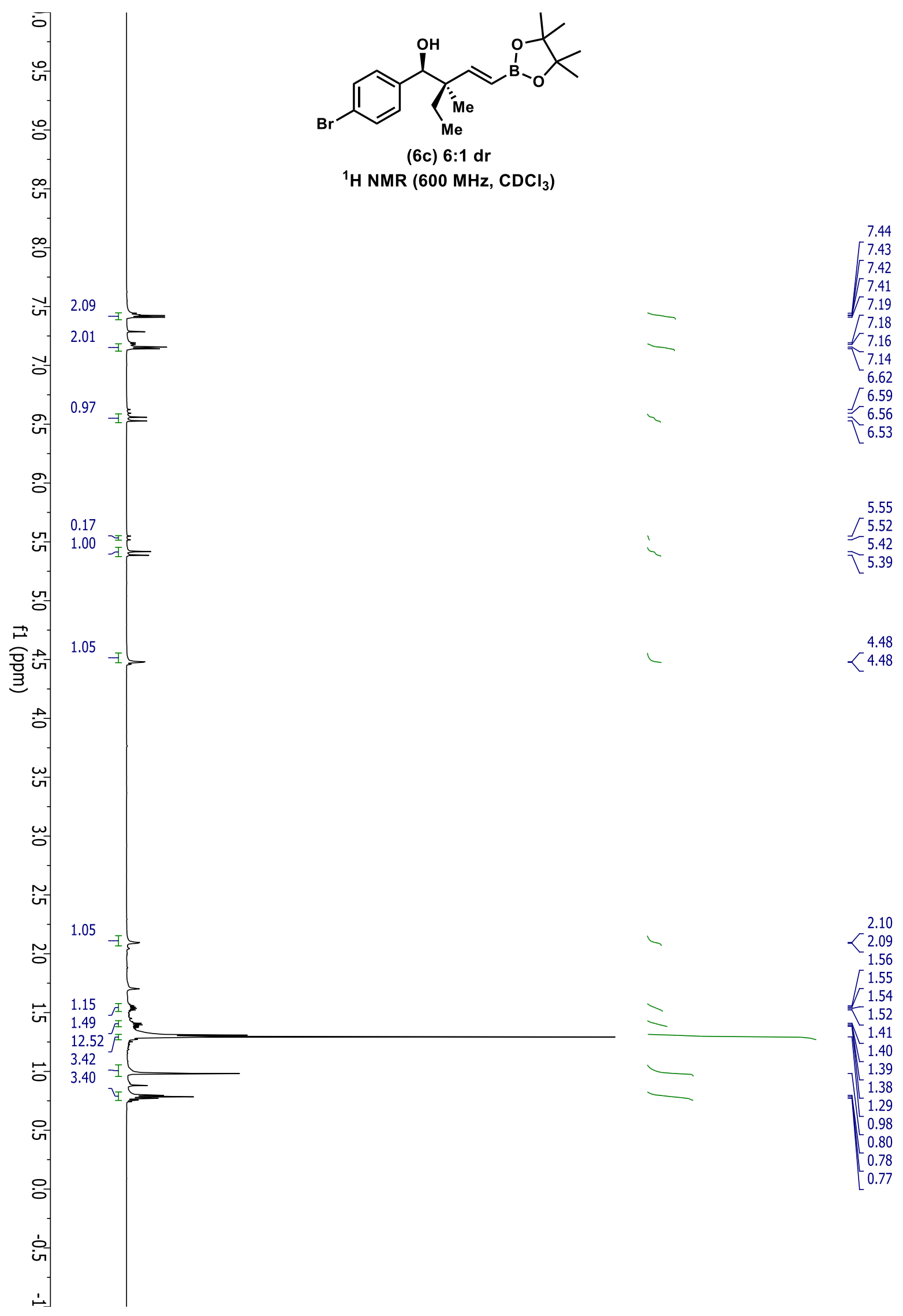




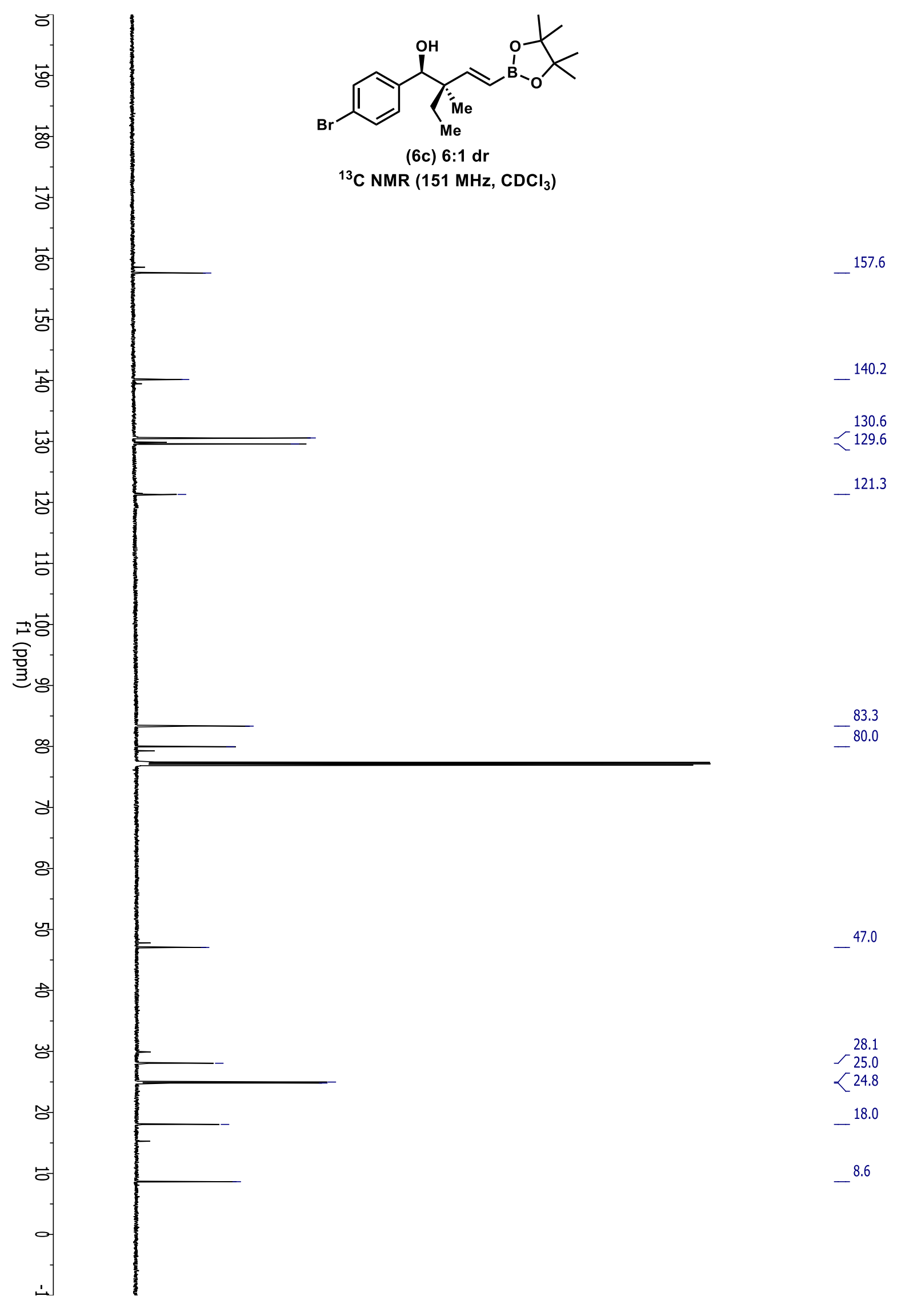




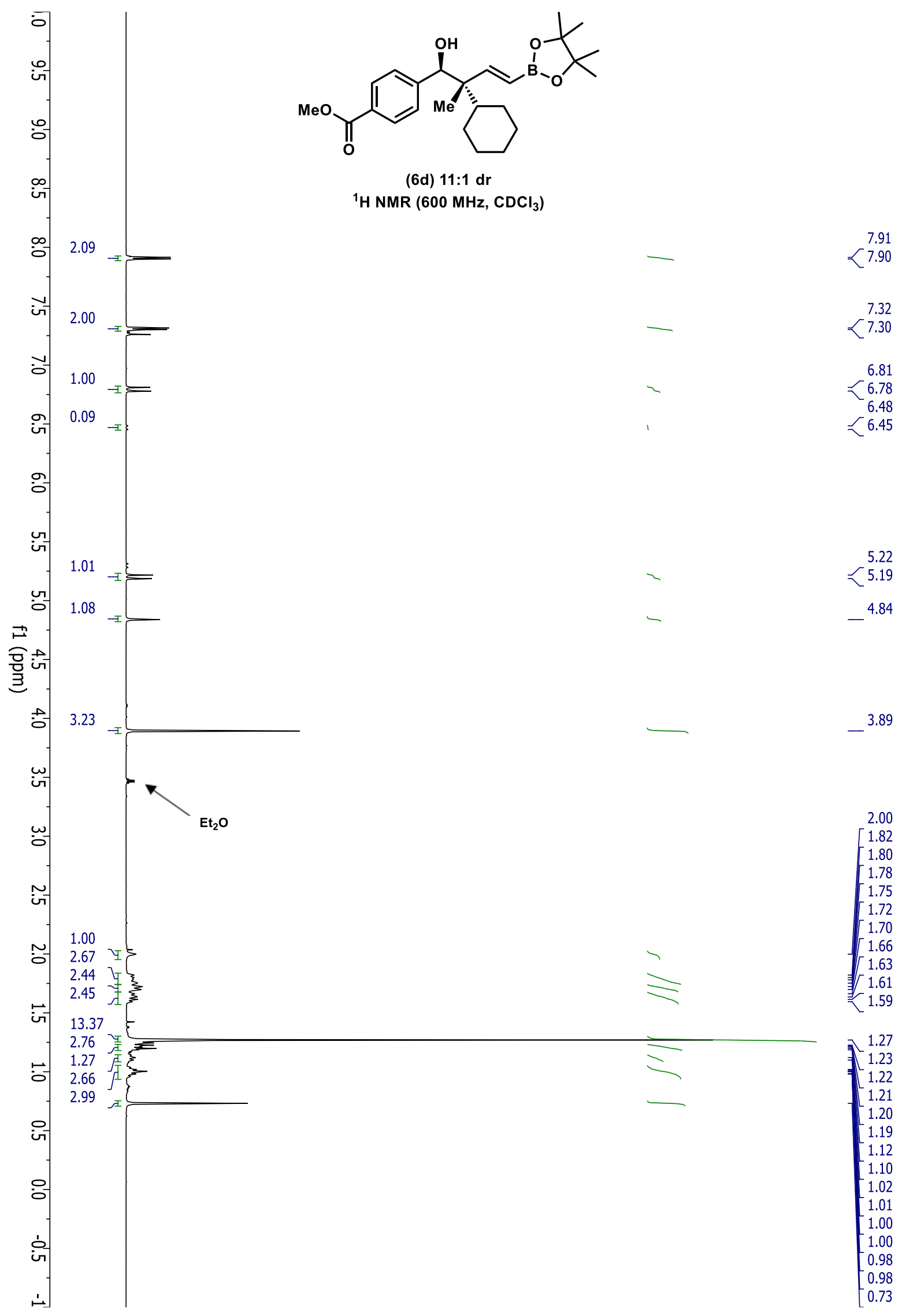




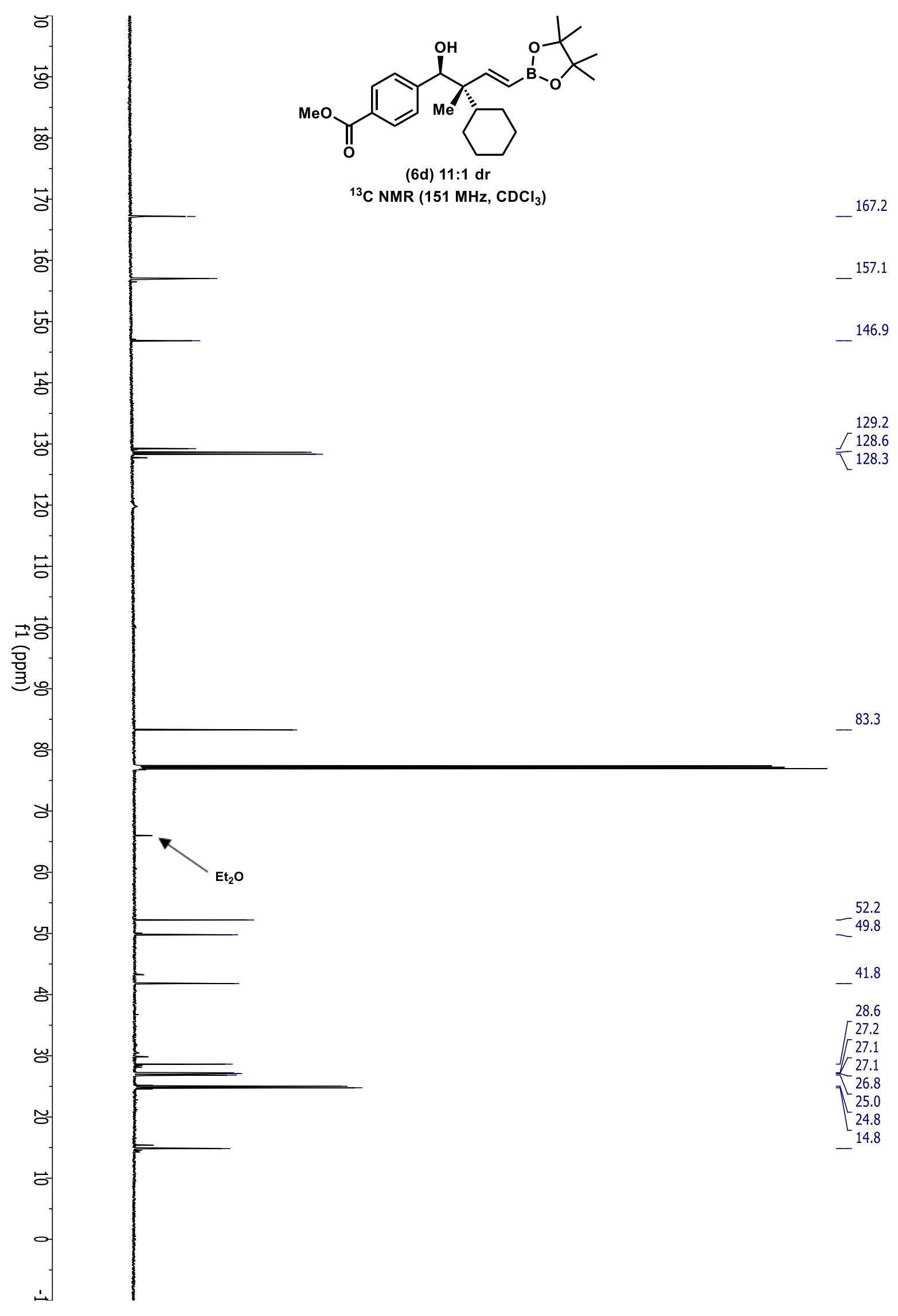




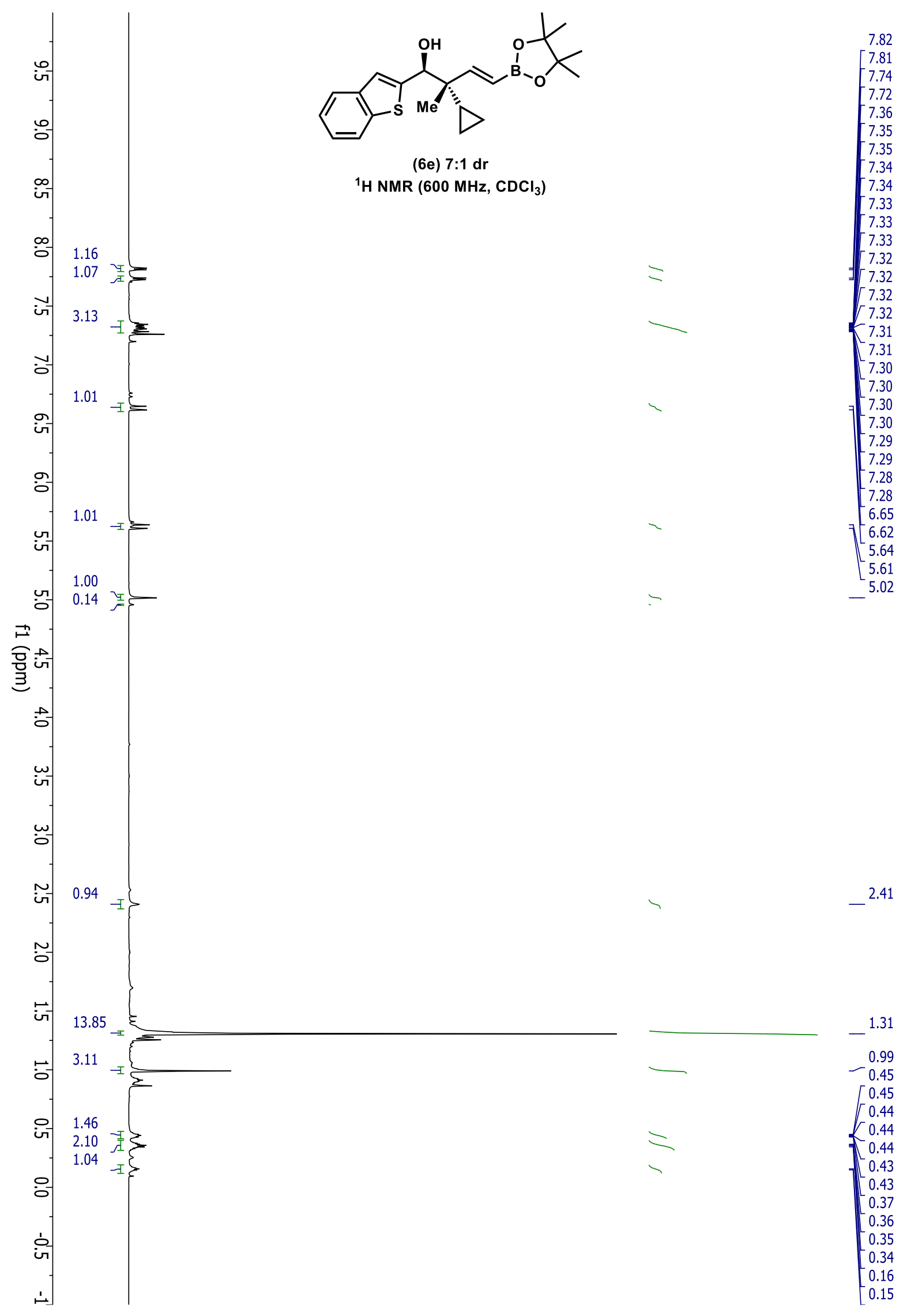




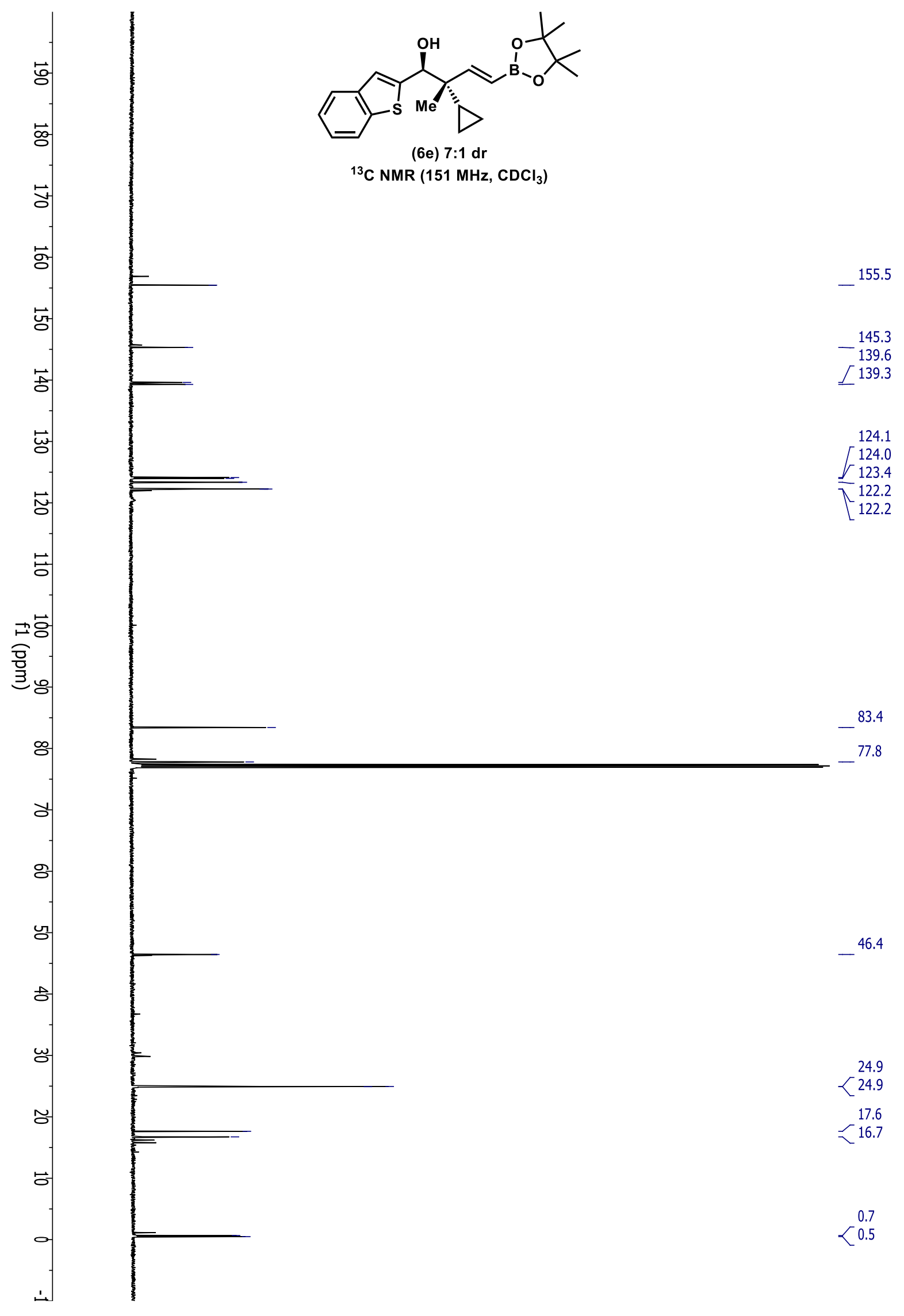




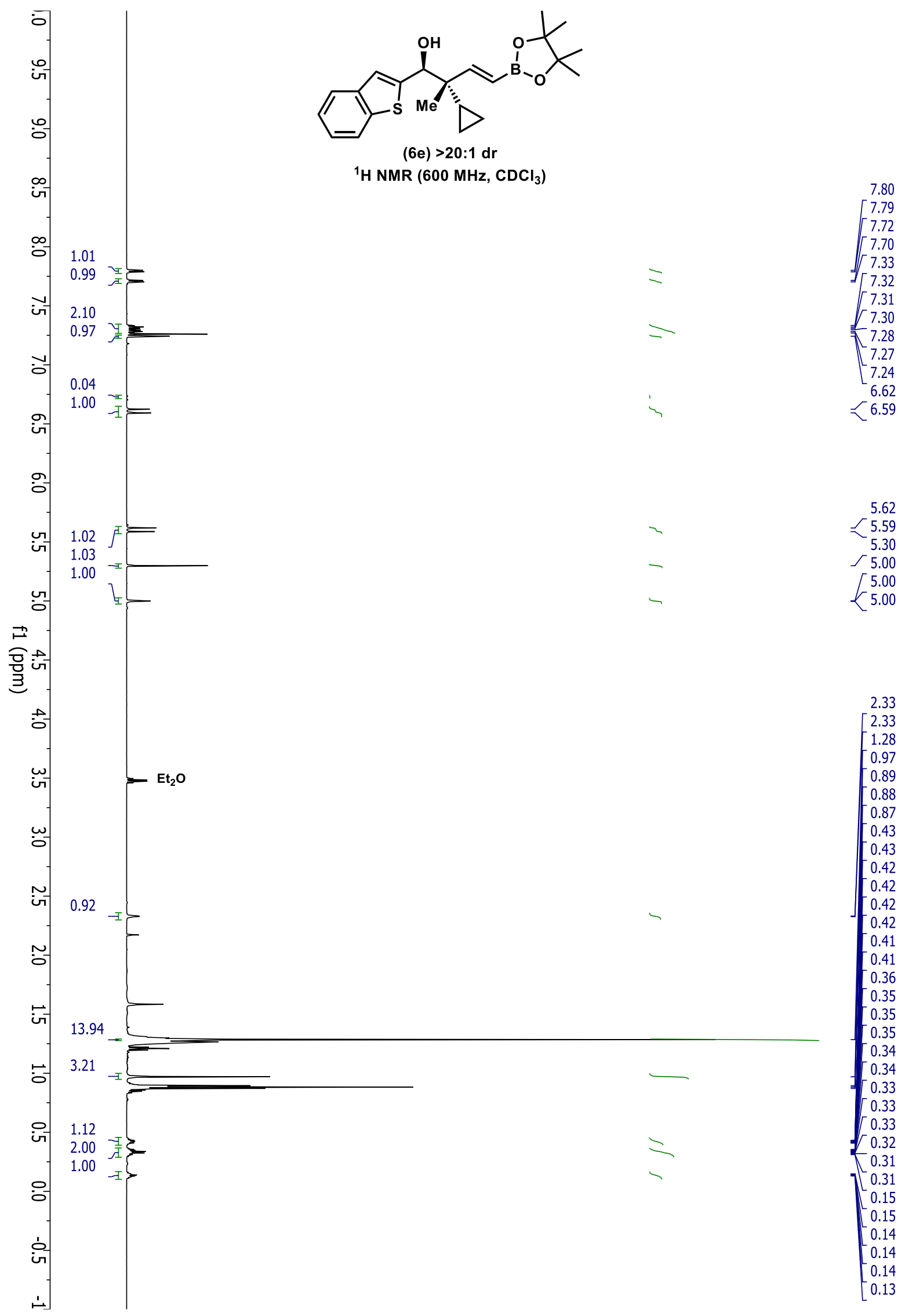




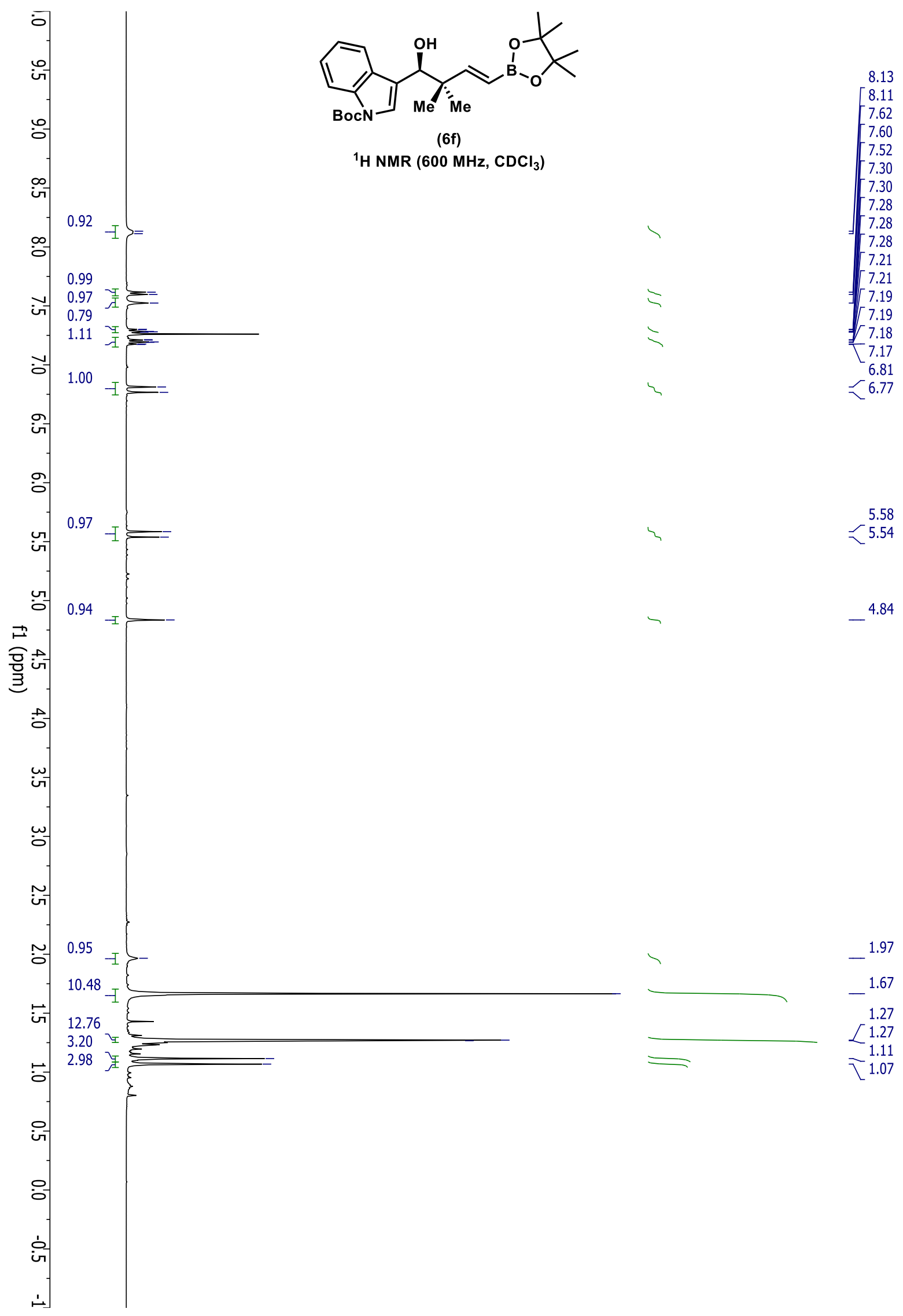




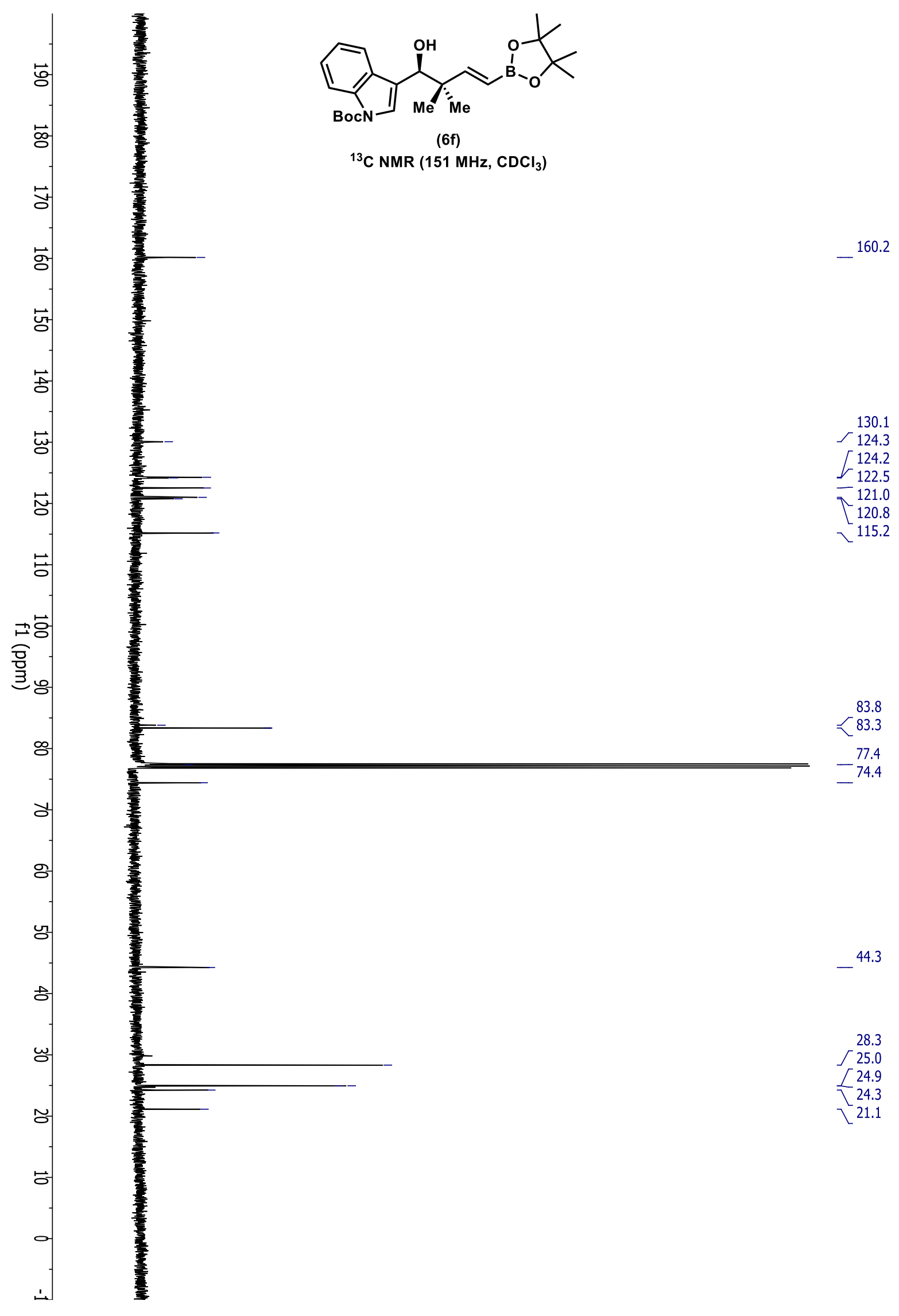



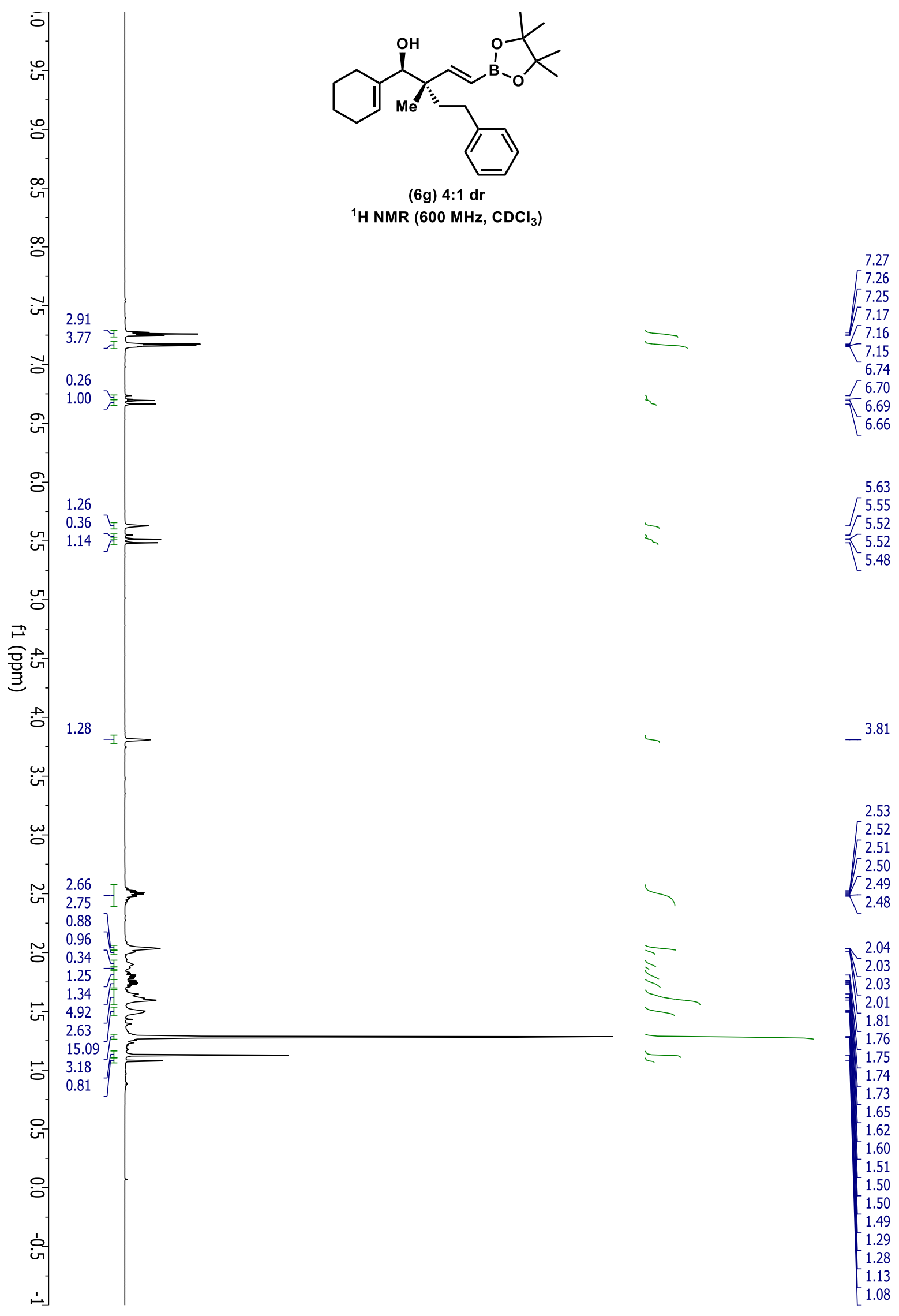


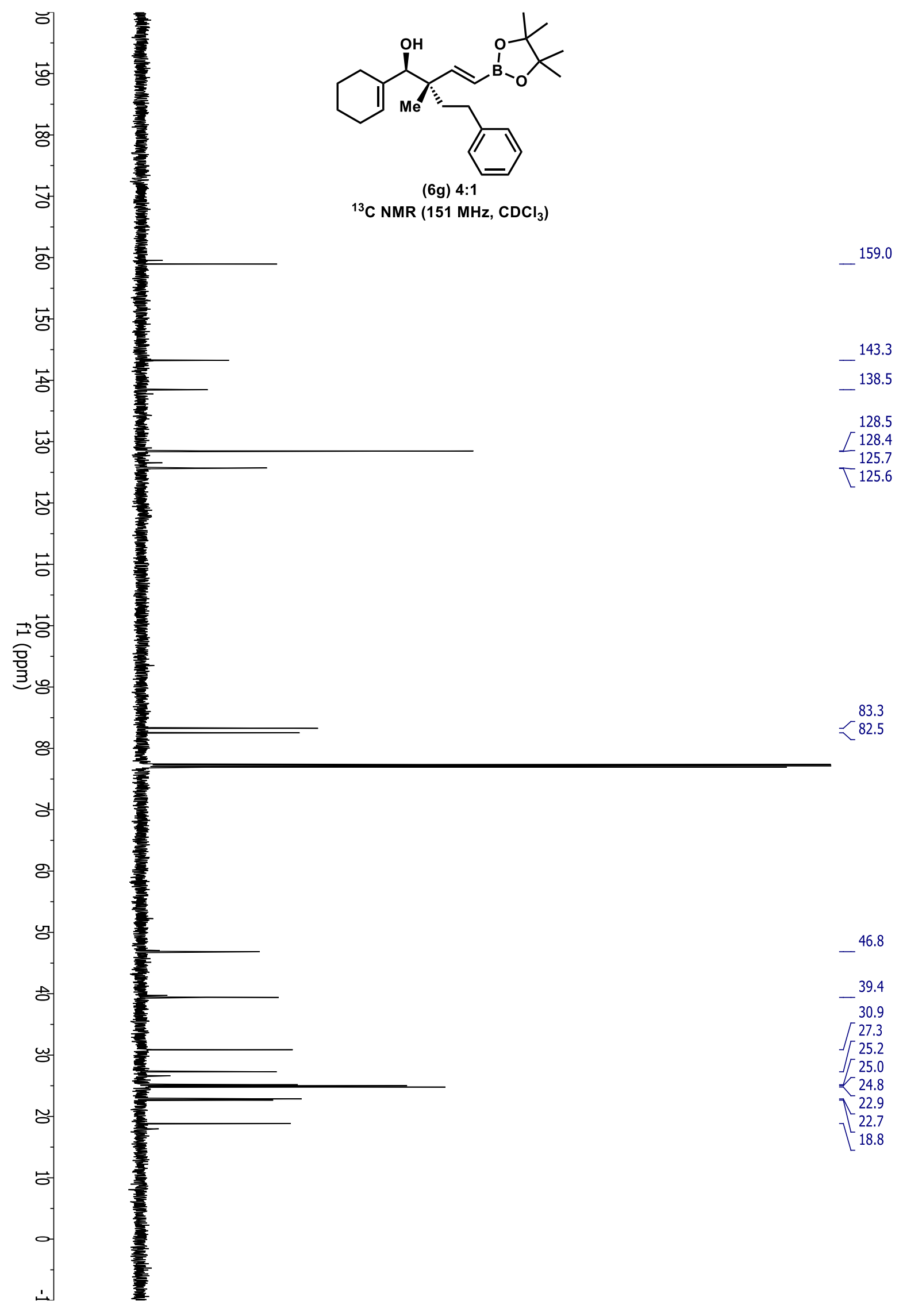




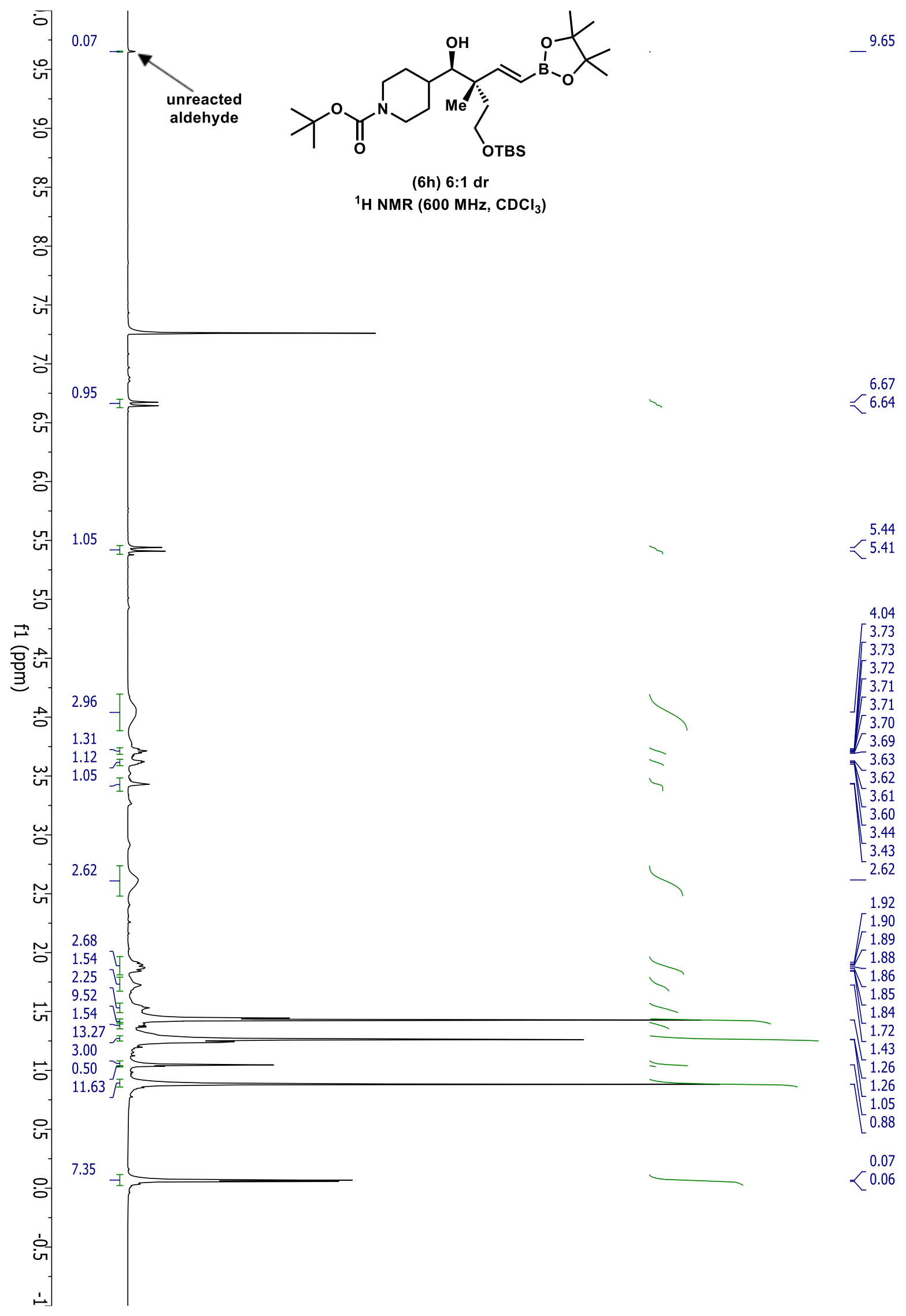




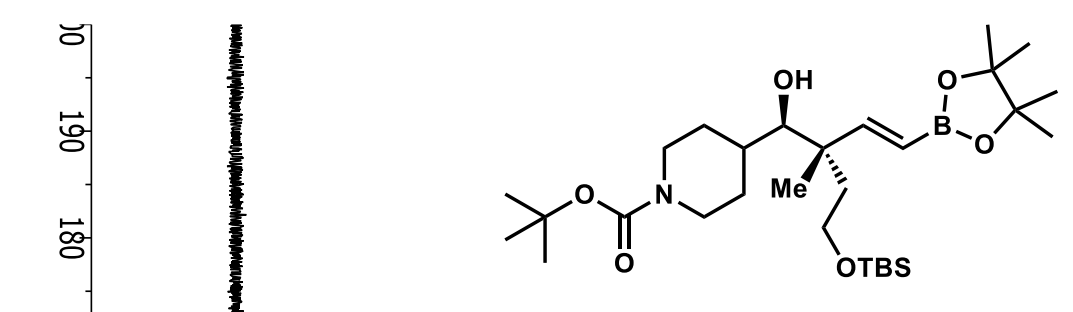

(6h) 6:1 dr

${ }^{13} \mathrm{C}$ NMR (151 MHz, $\mathrm{CDCl}_{3}$ )

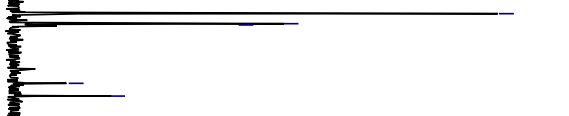




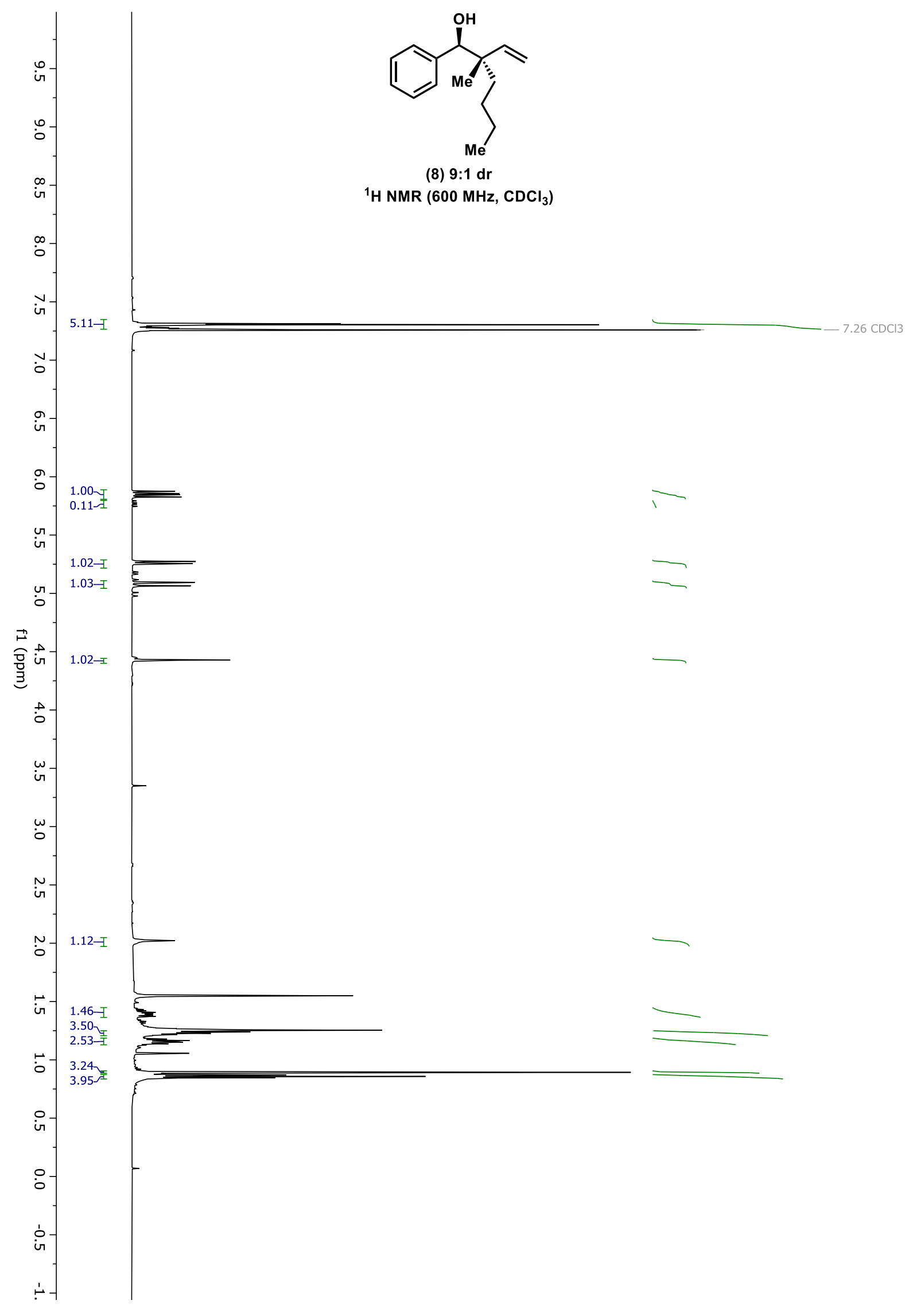




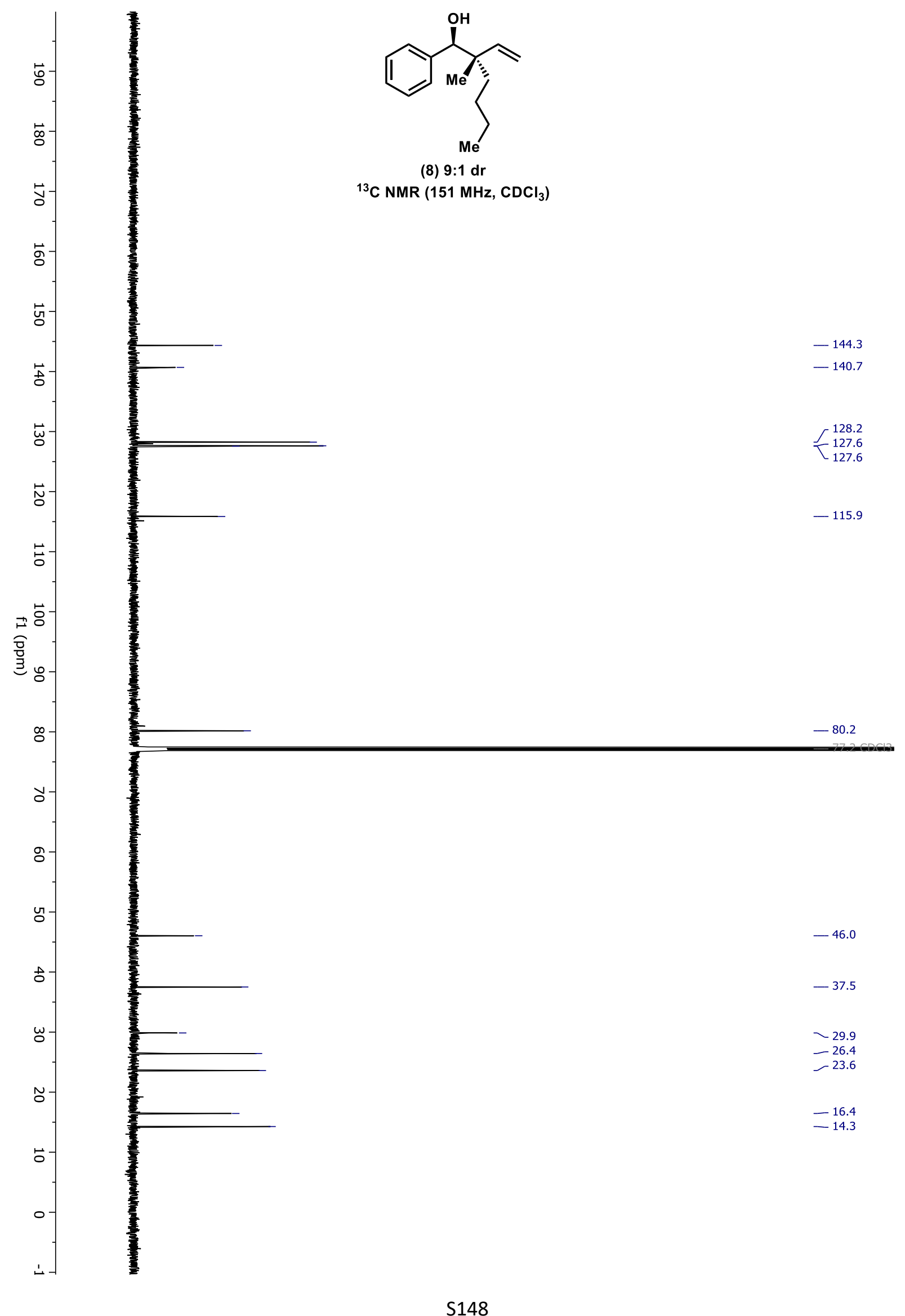




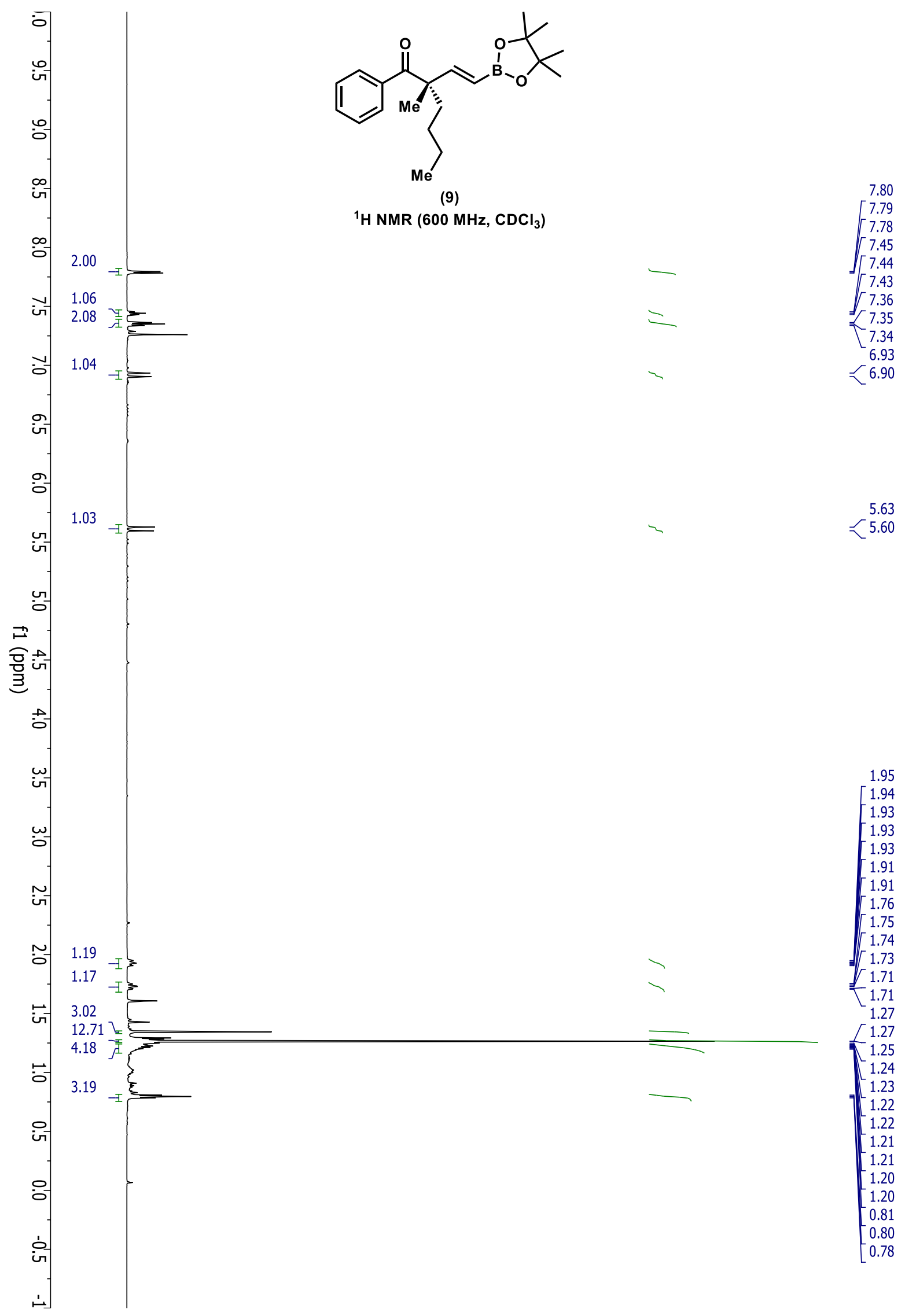




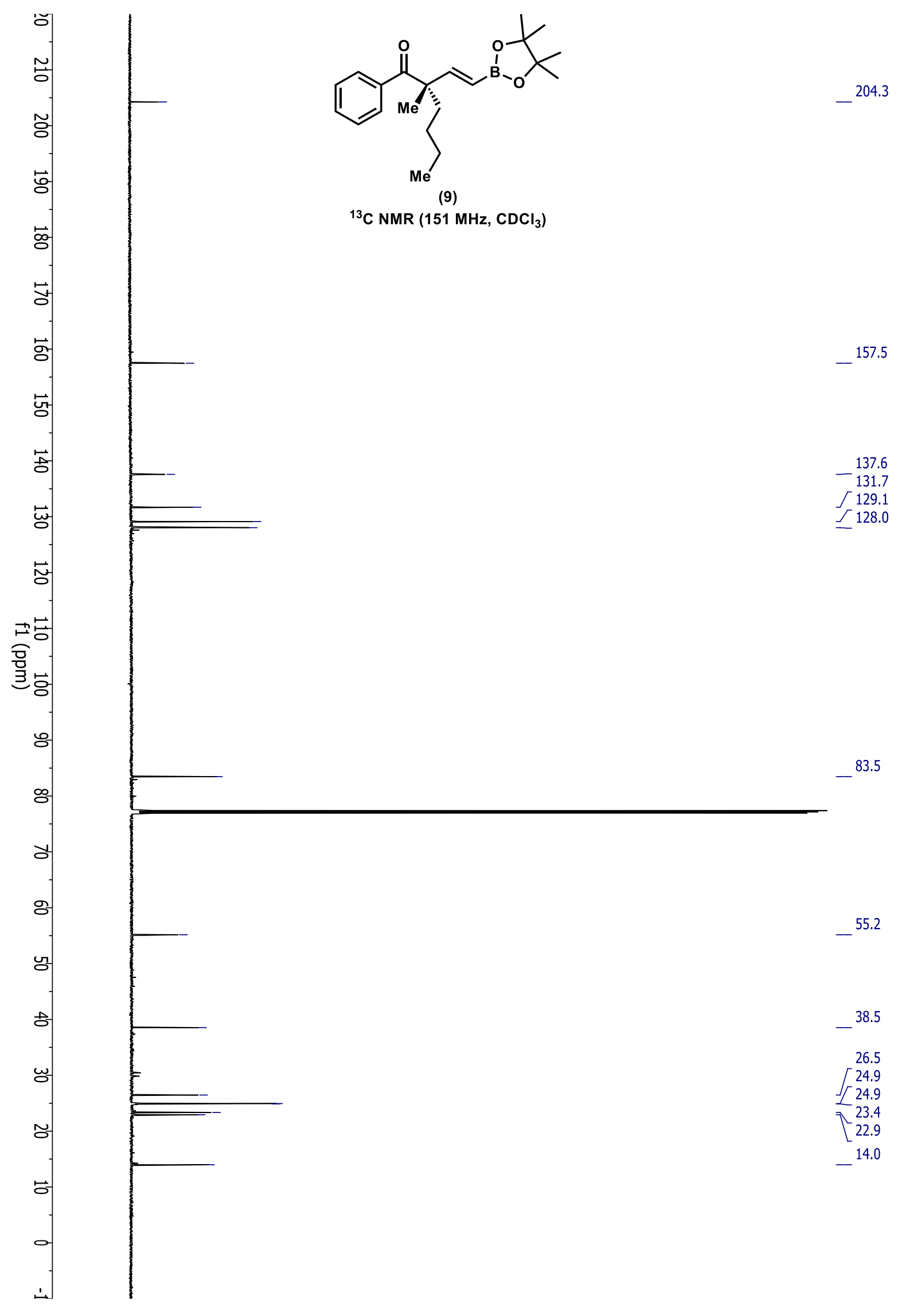




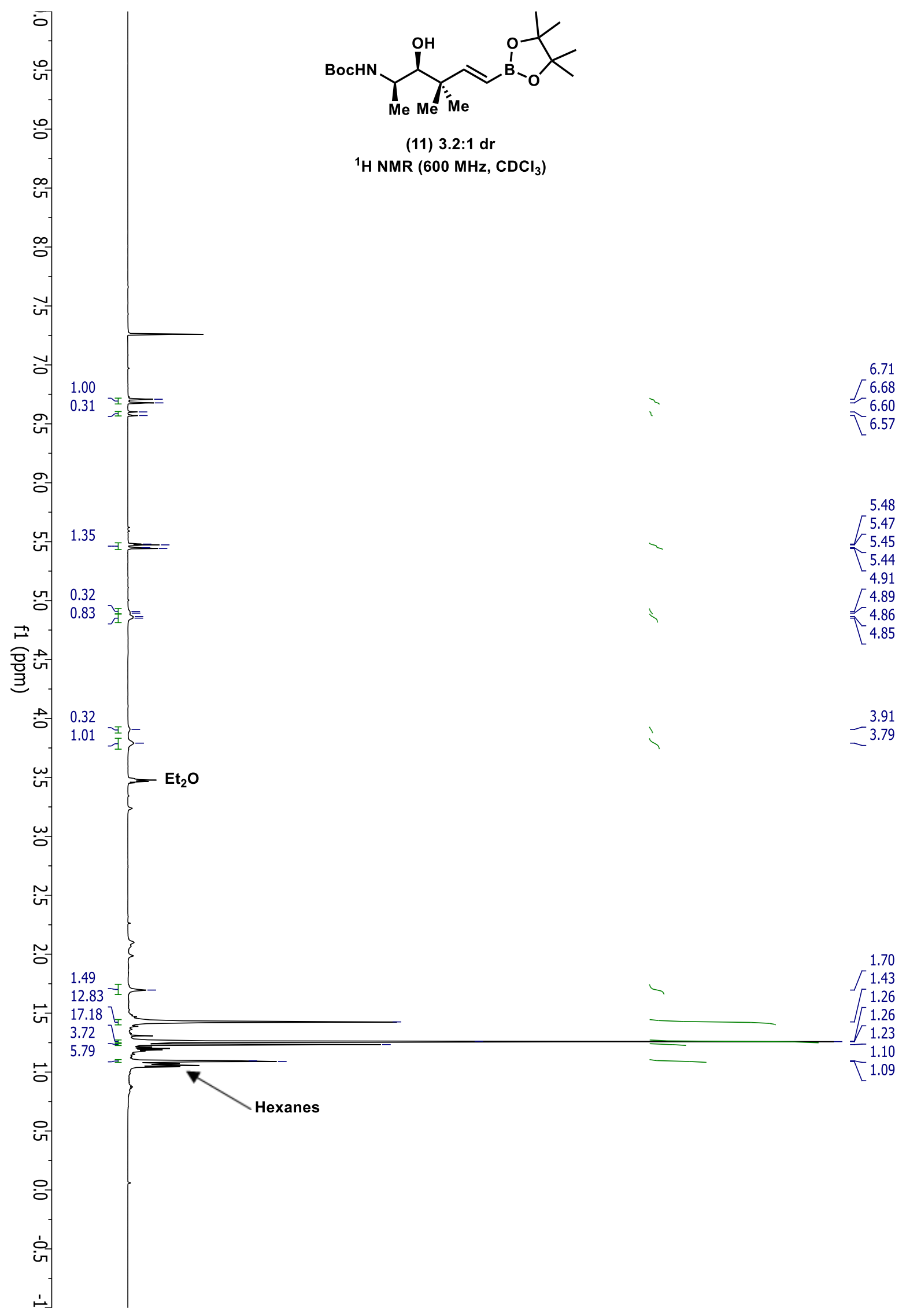




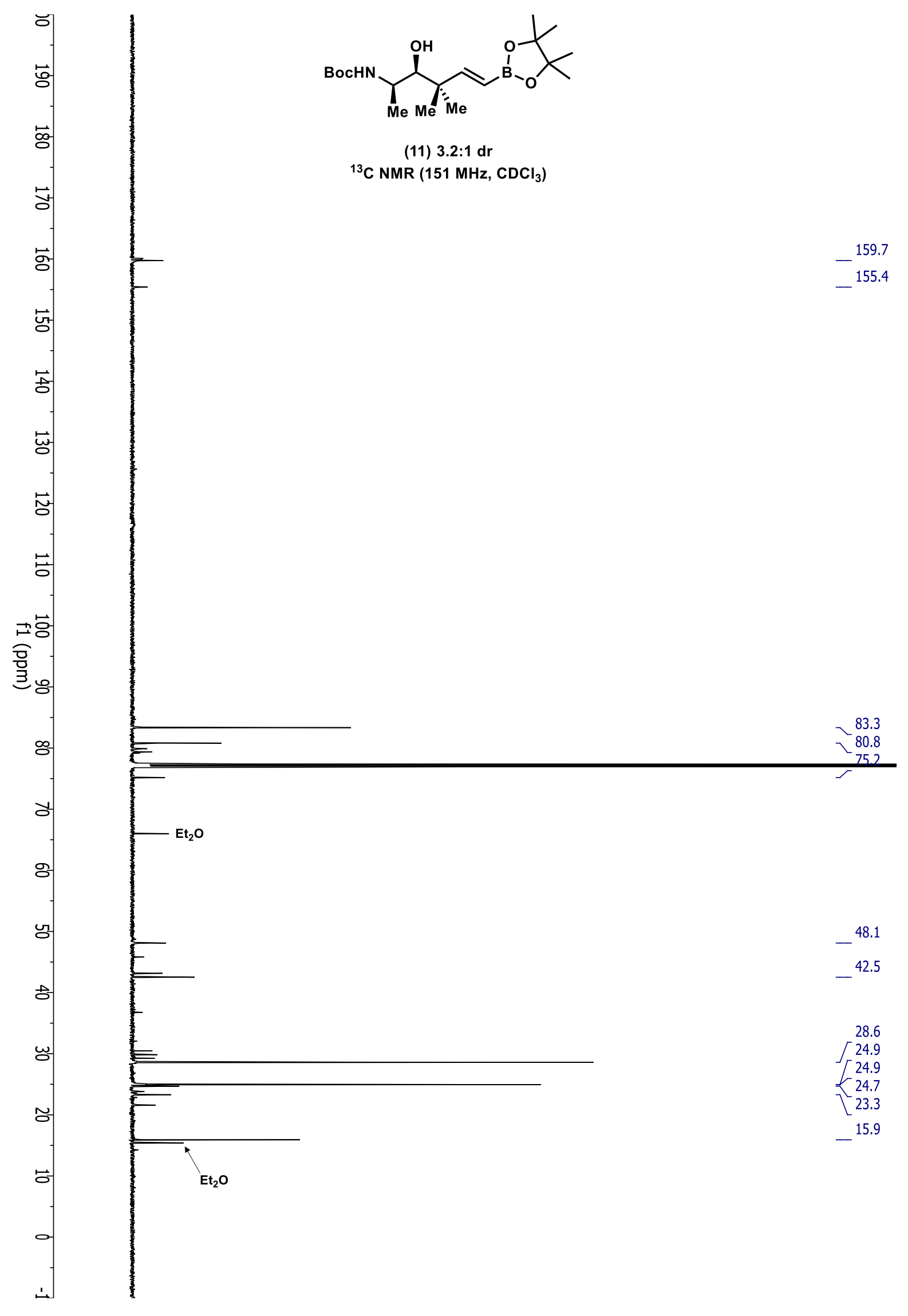




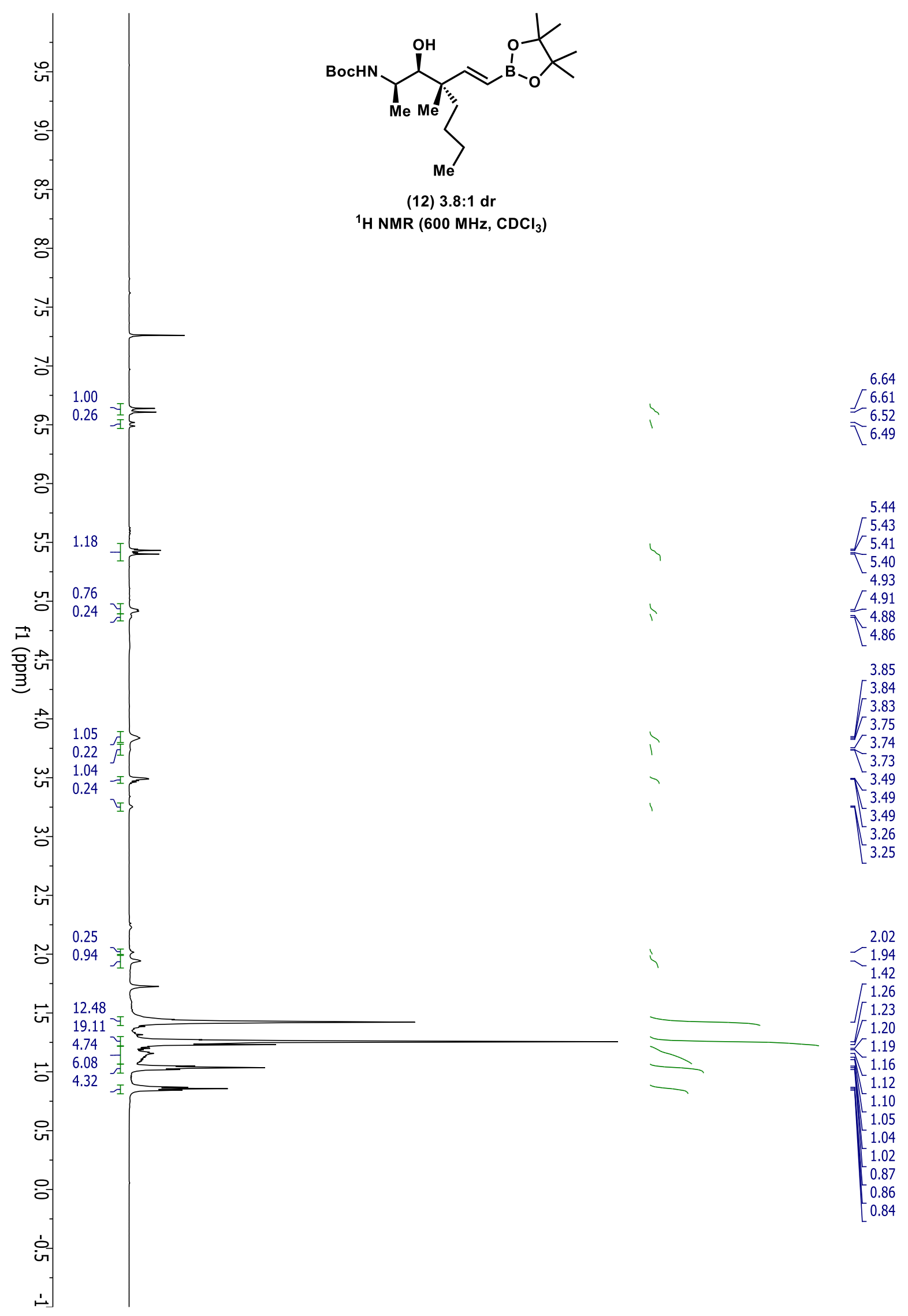




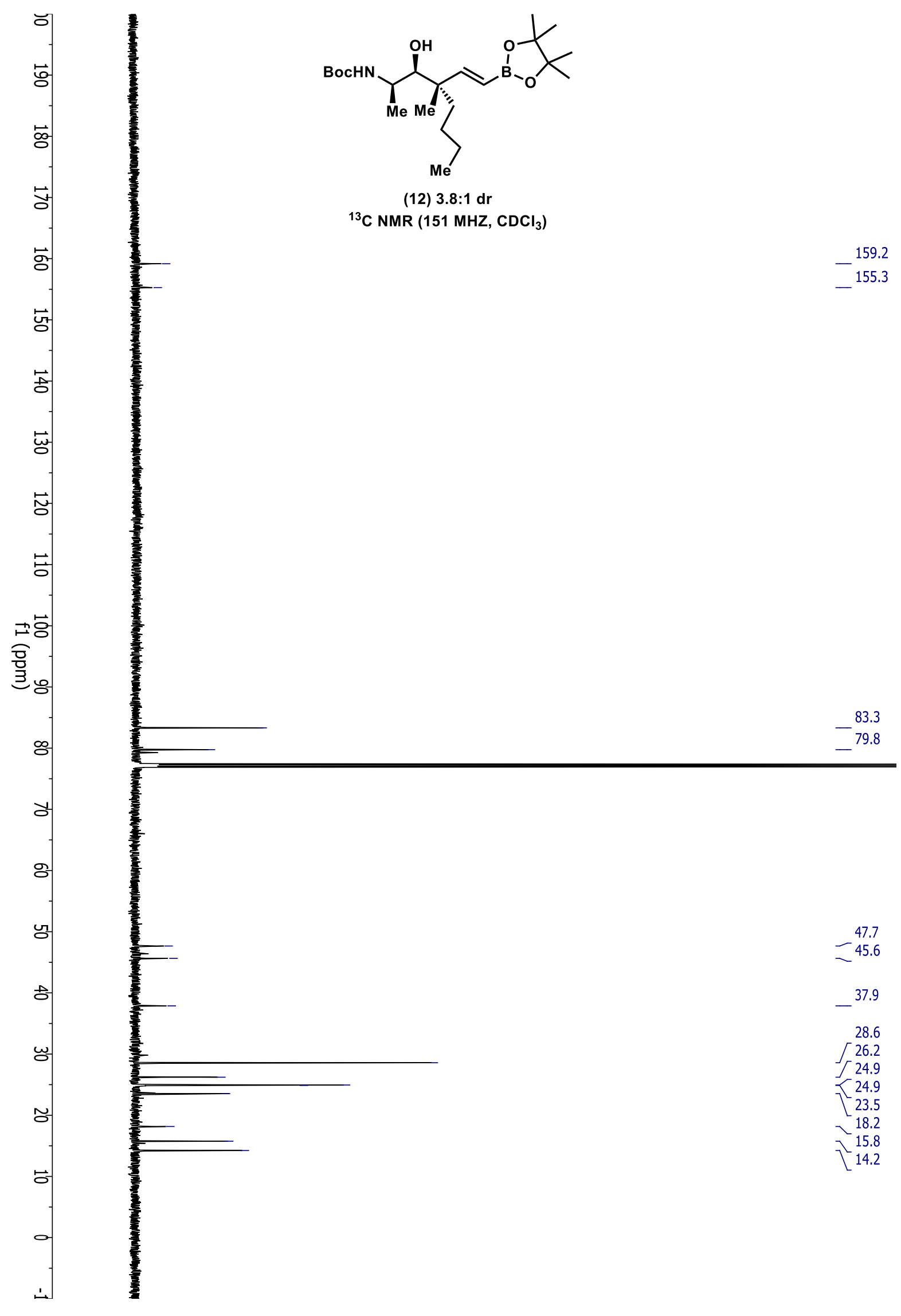



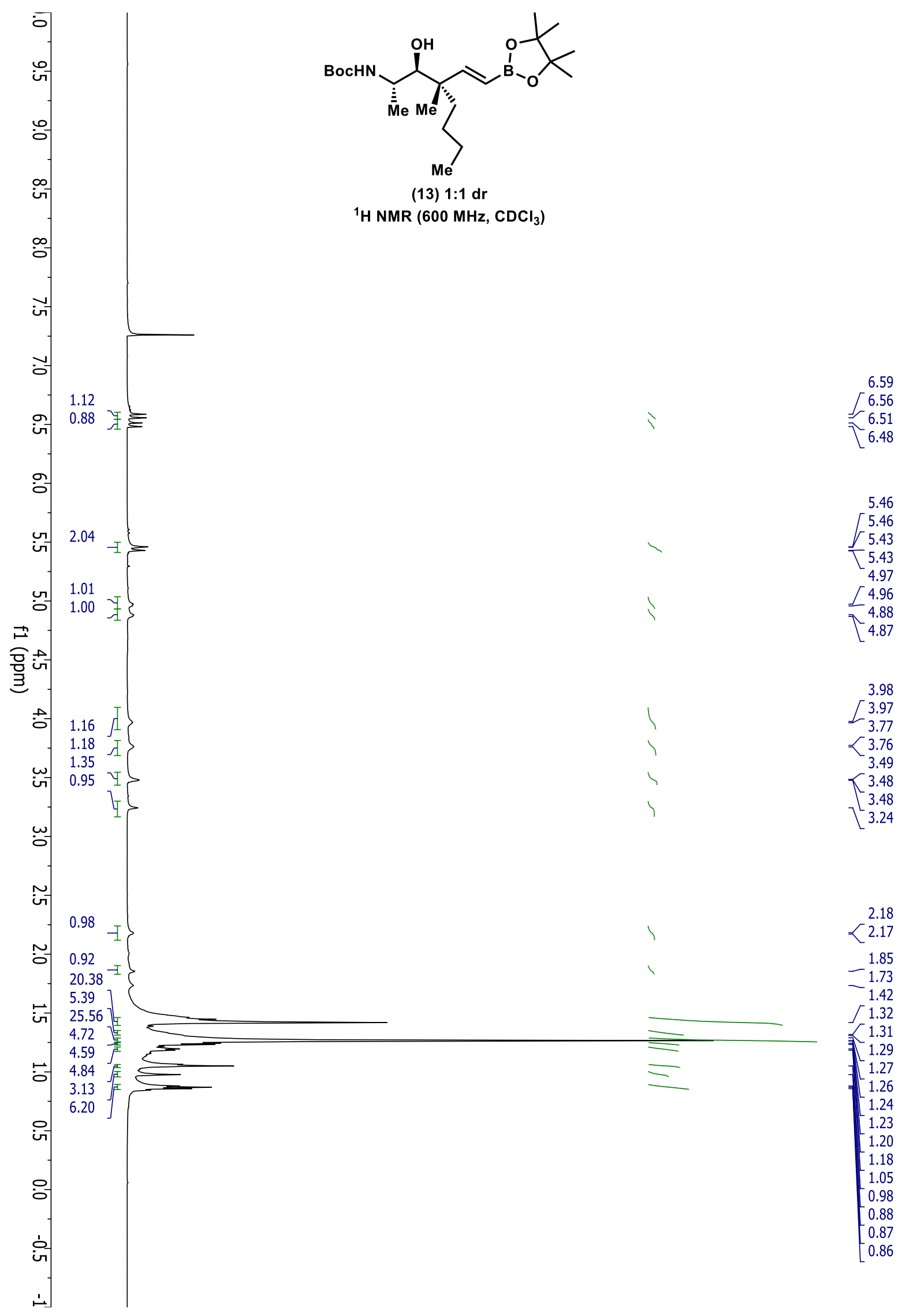

3.98

3.97
3.77

< 3.76

3.49

र 3.48

3.48

3.24

2.18

$\{2.17$

1.85

$-1.73$

$\sqrt{1.32}$

1.31

1.29

1.27

1.26

1.24
1.23

1.23

1.20
1.18

1.05

1.05
0.98

0.88

0.87
-0.86 


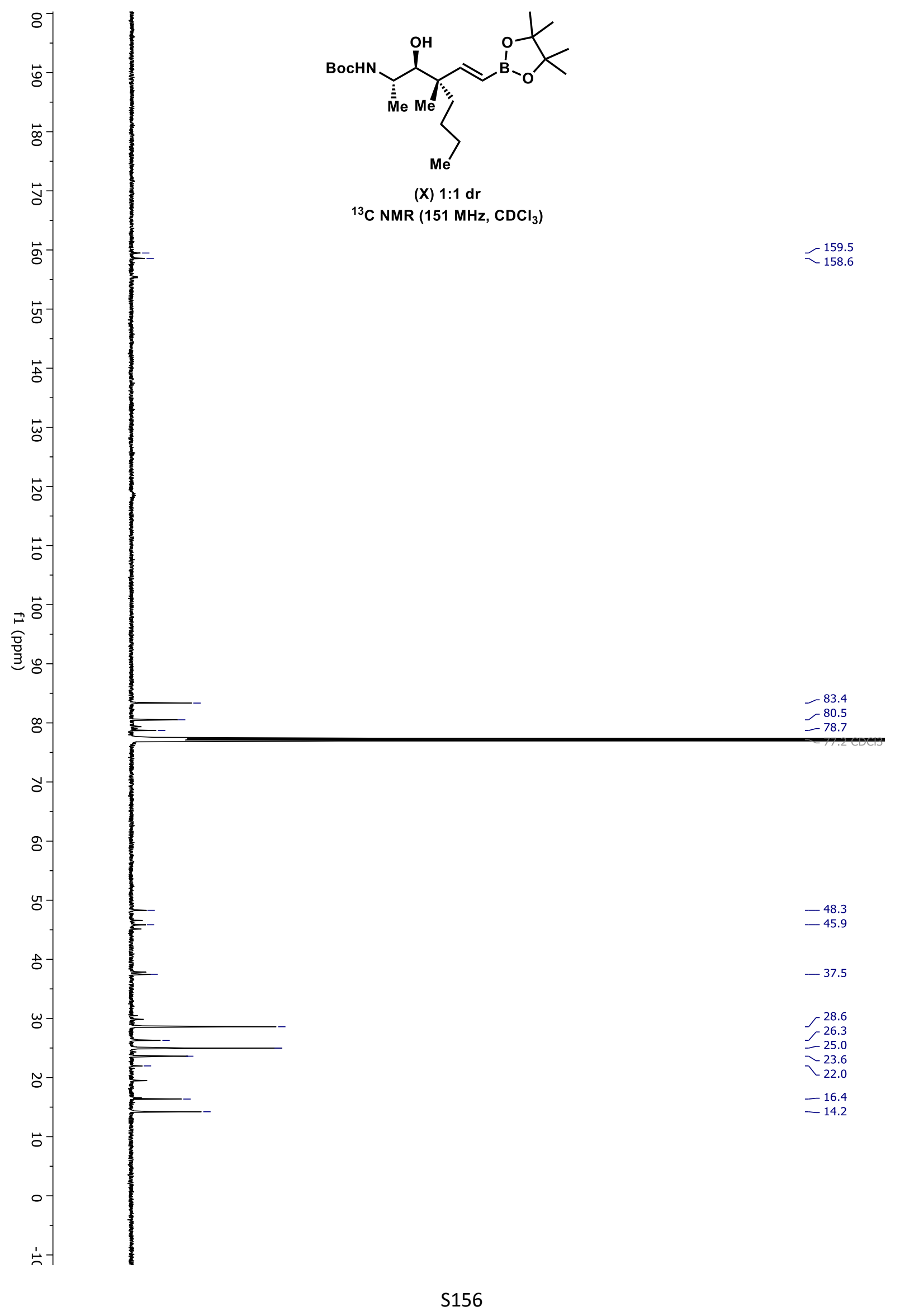




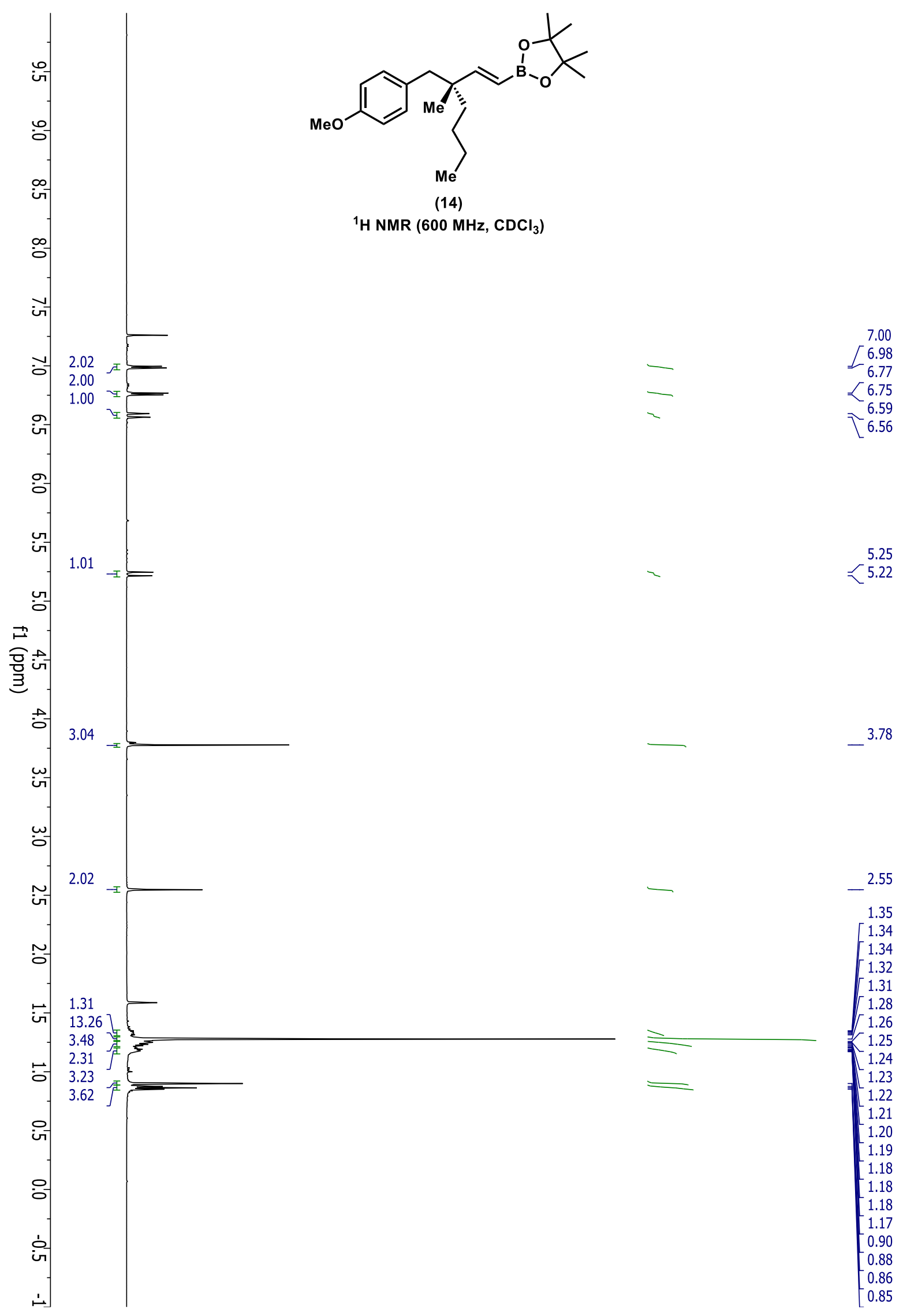




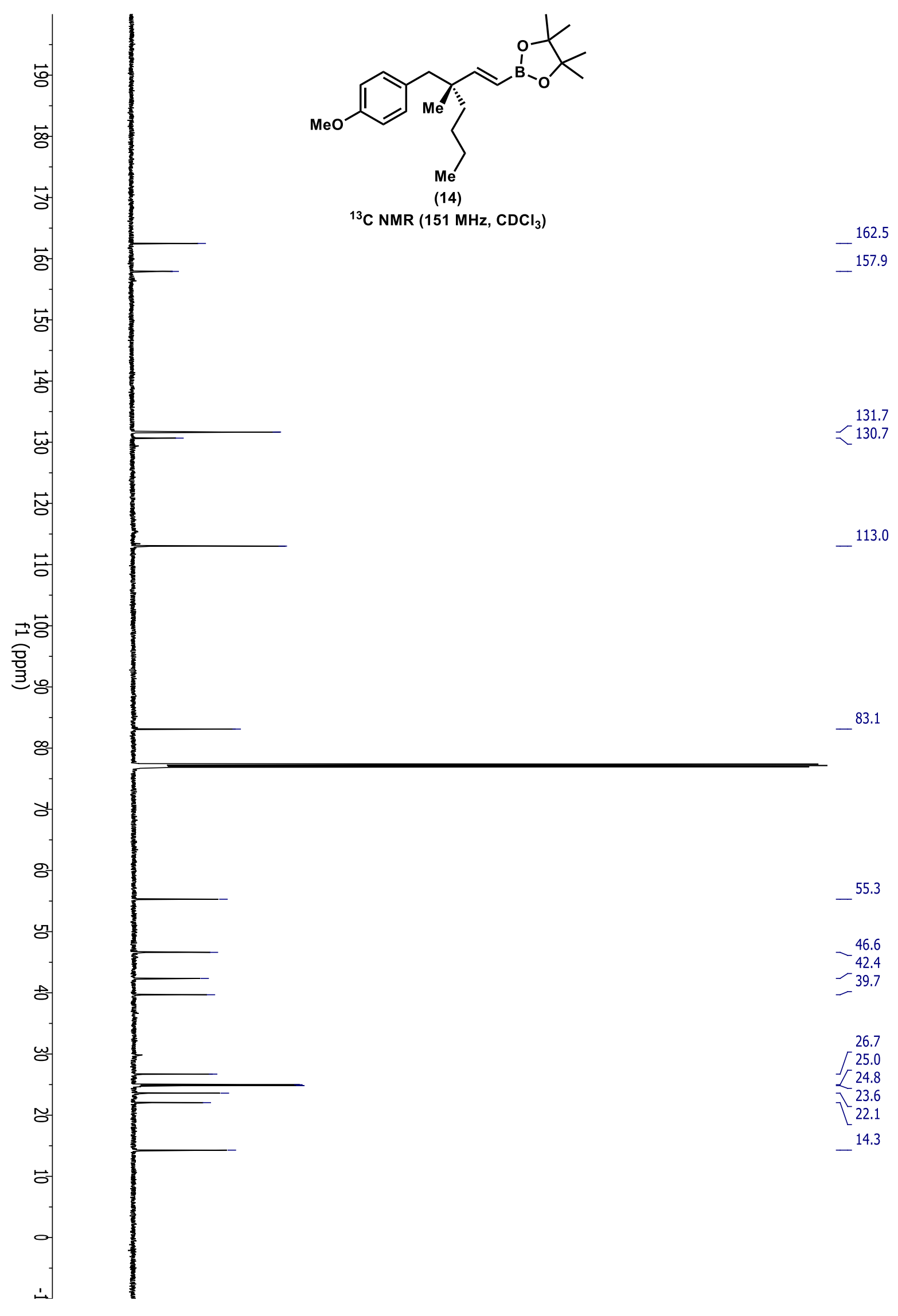




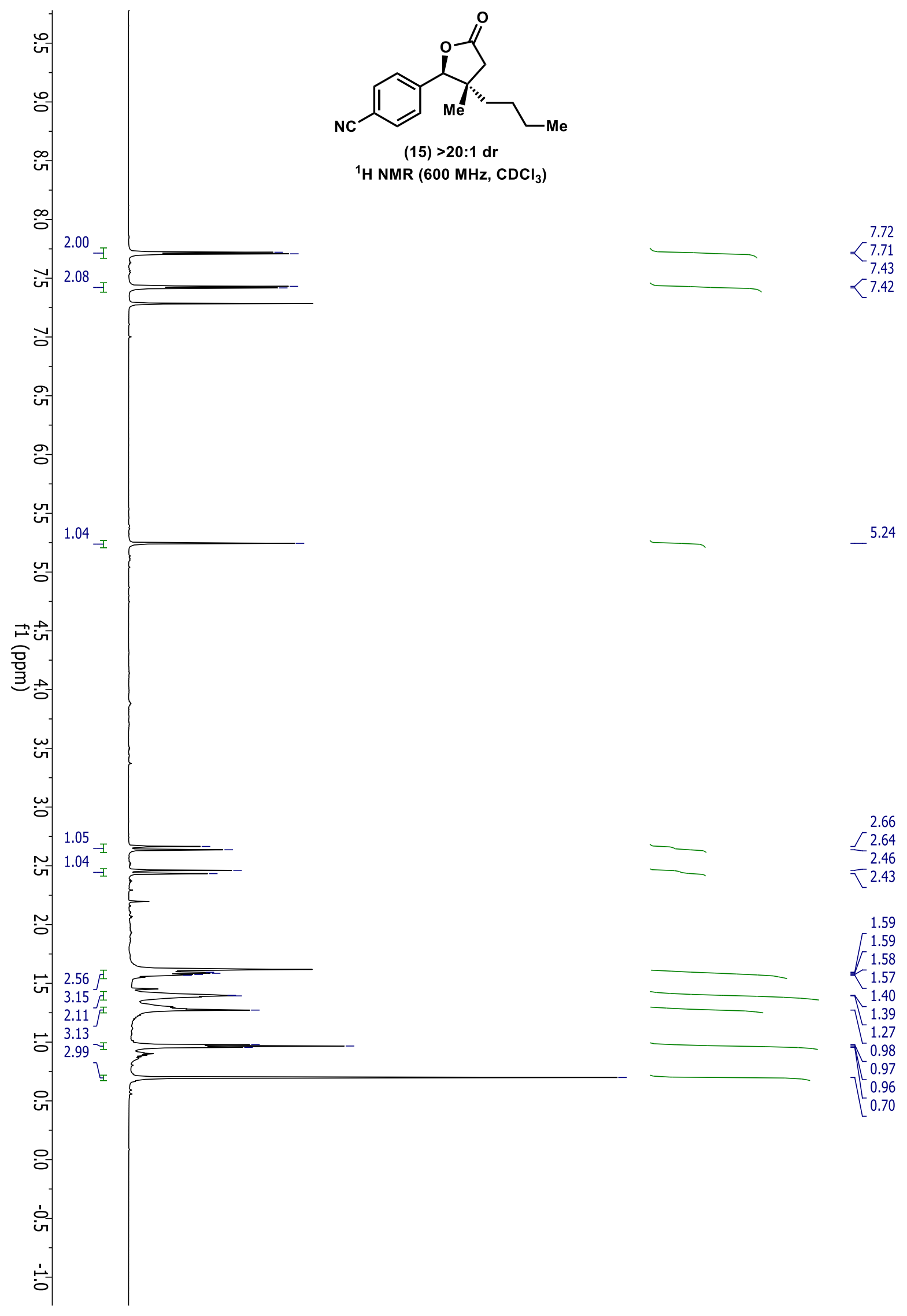




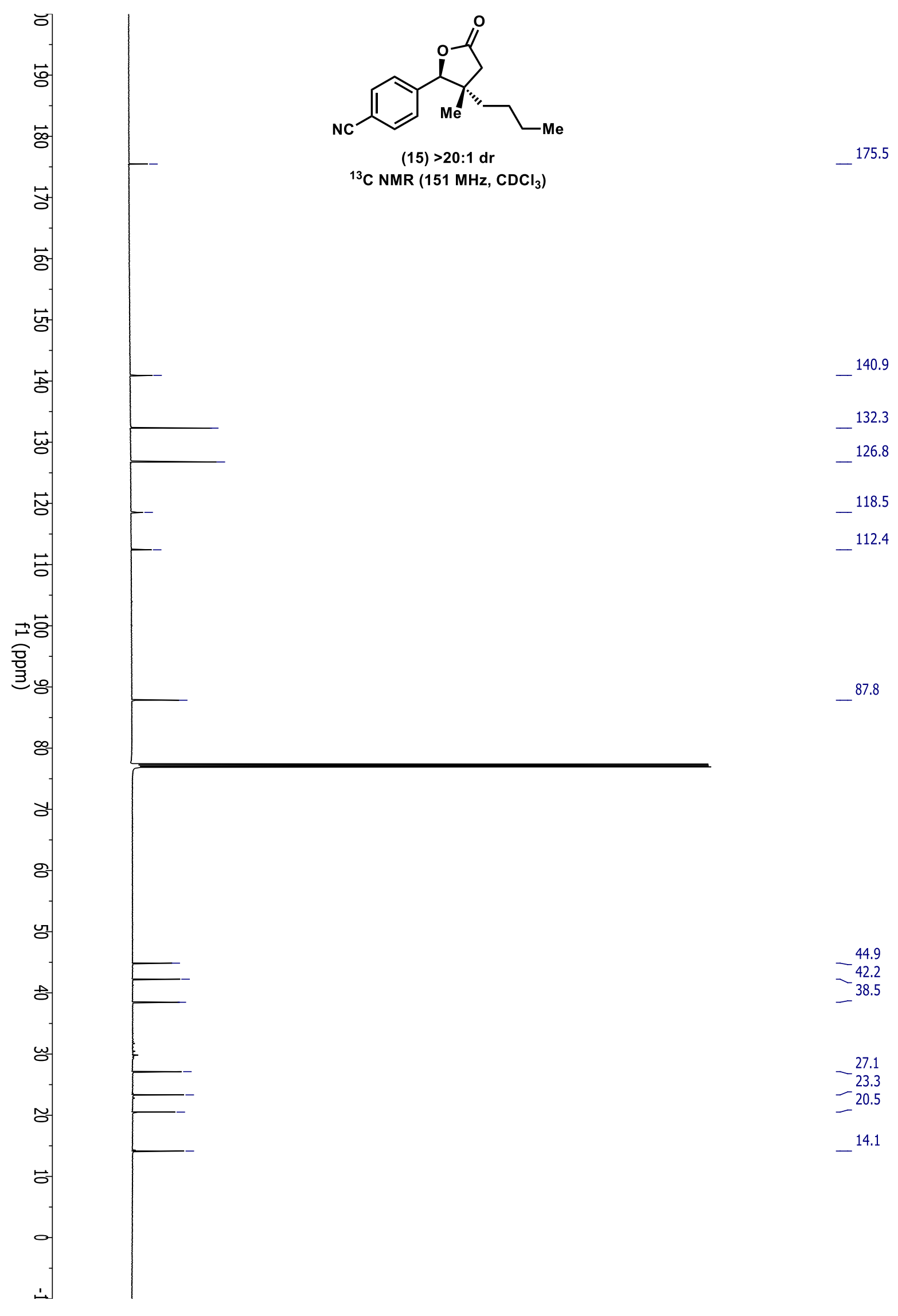




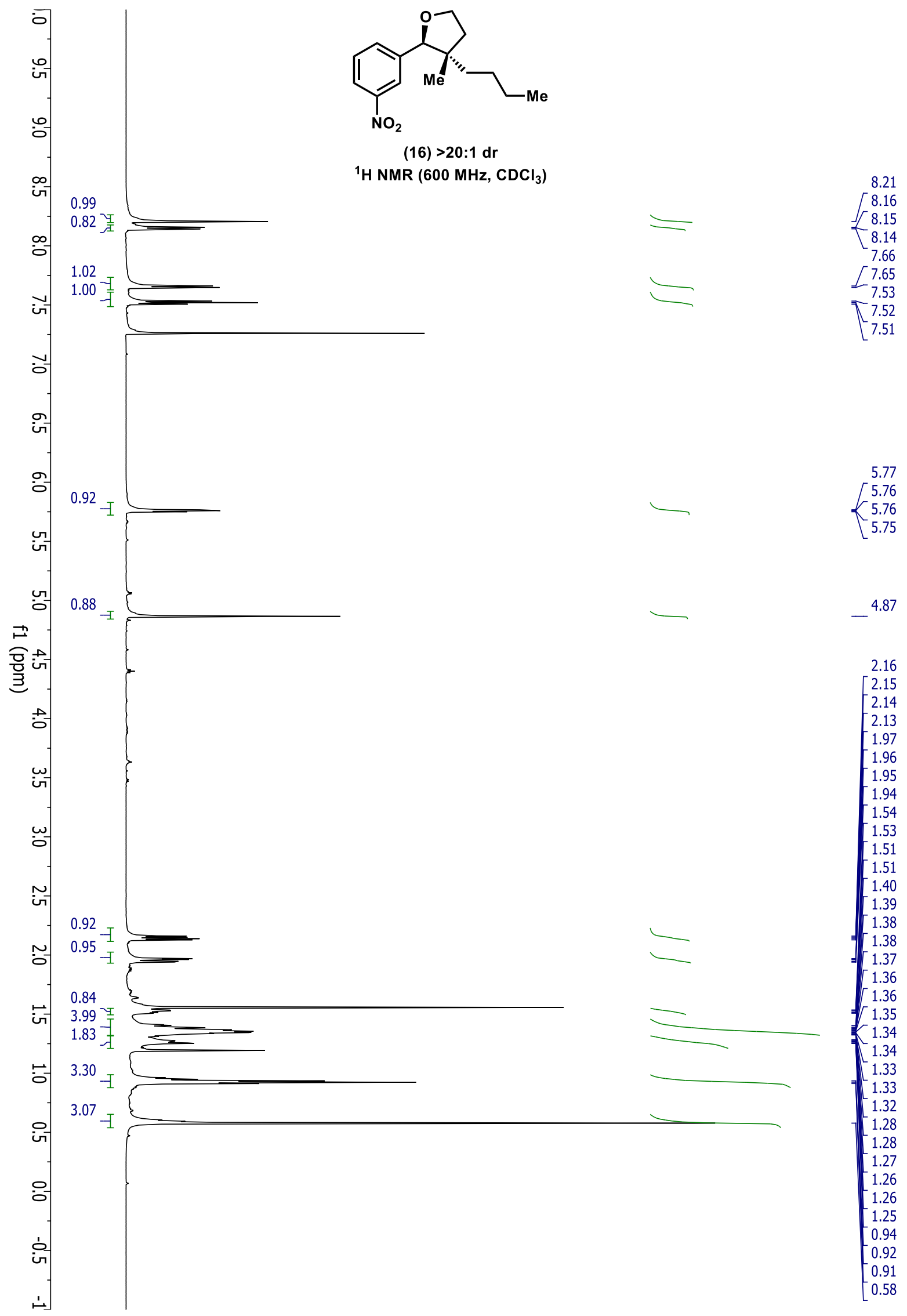




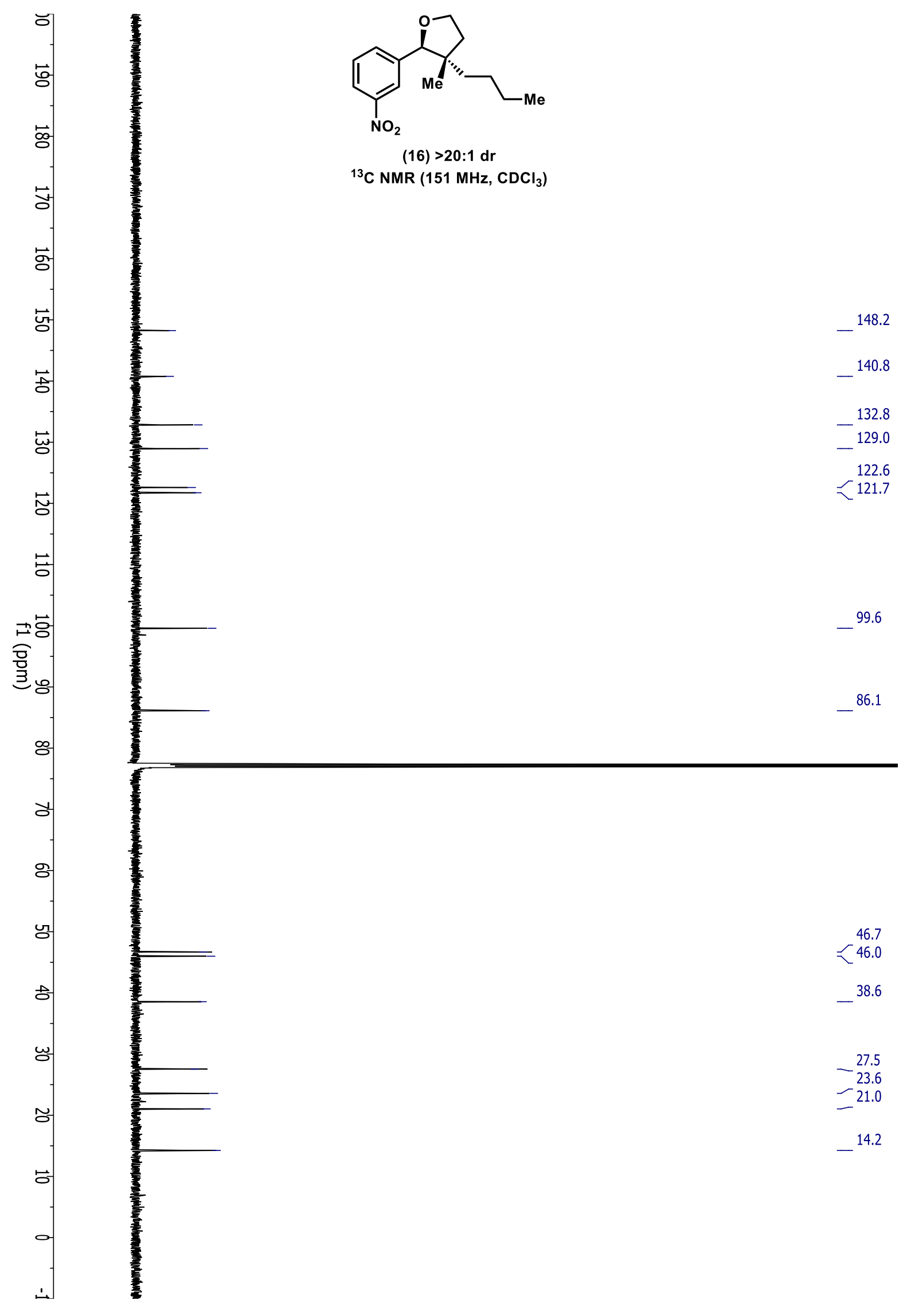




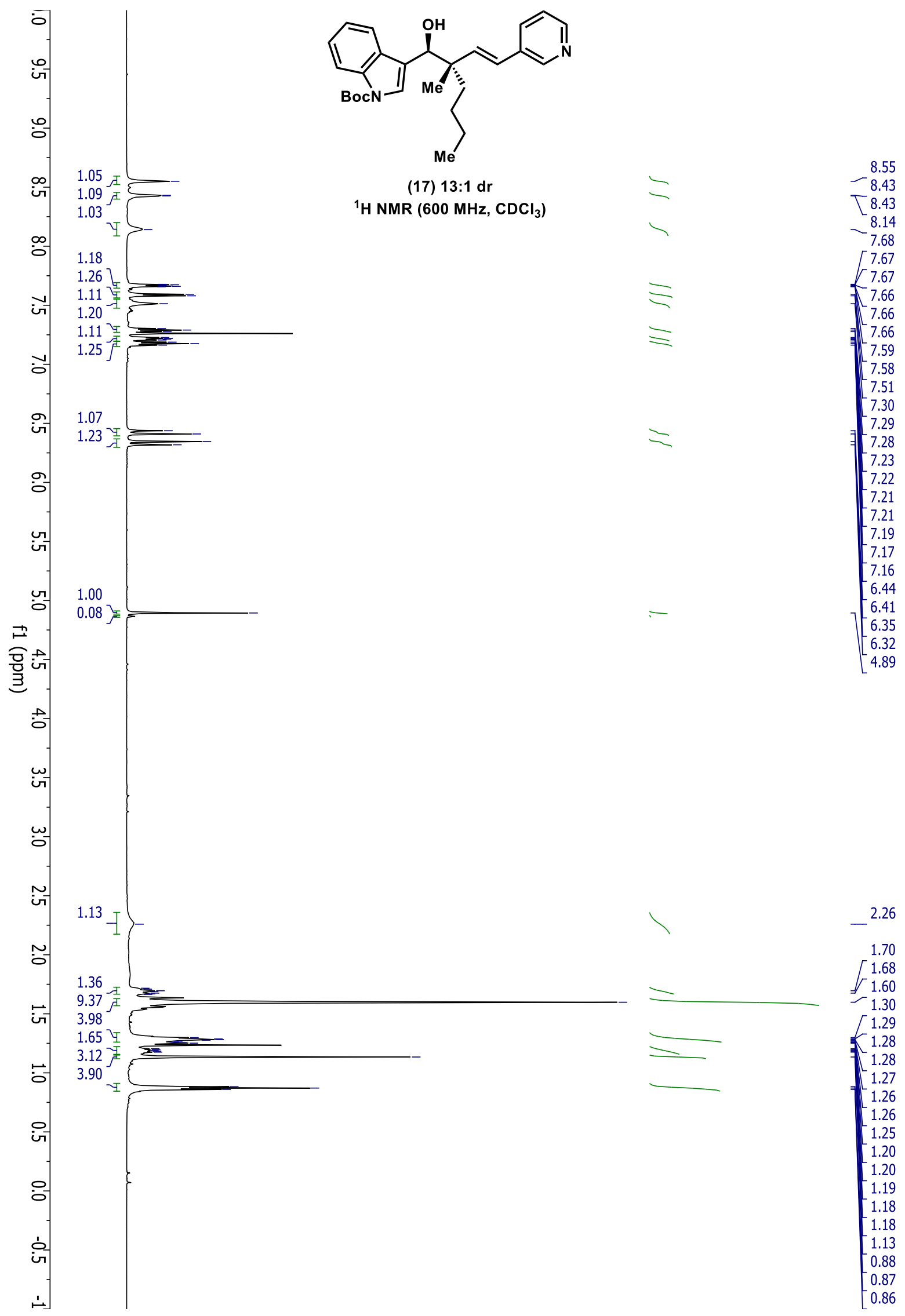




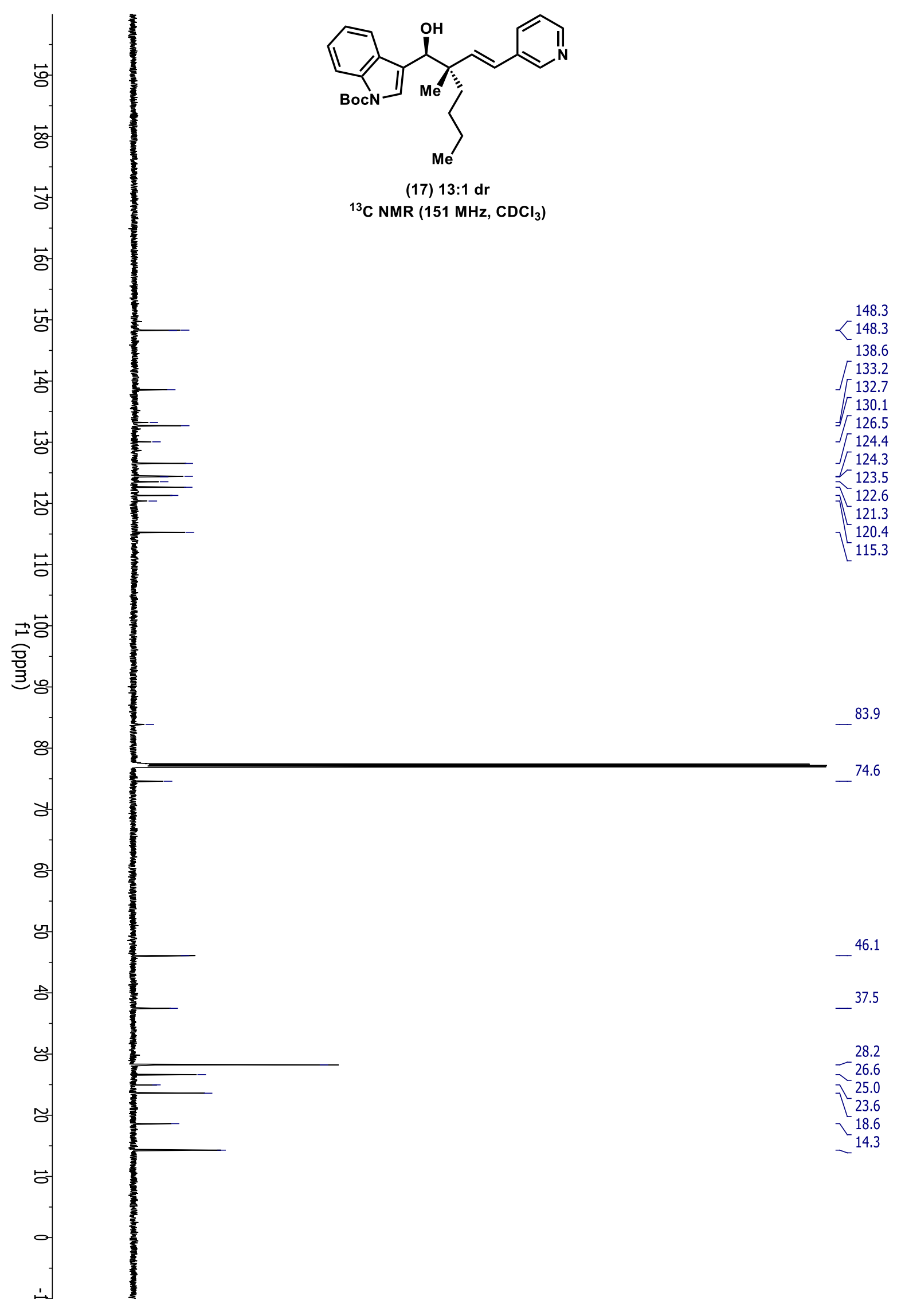




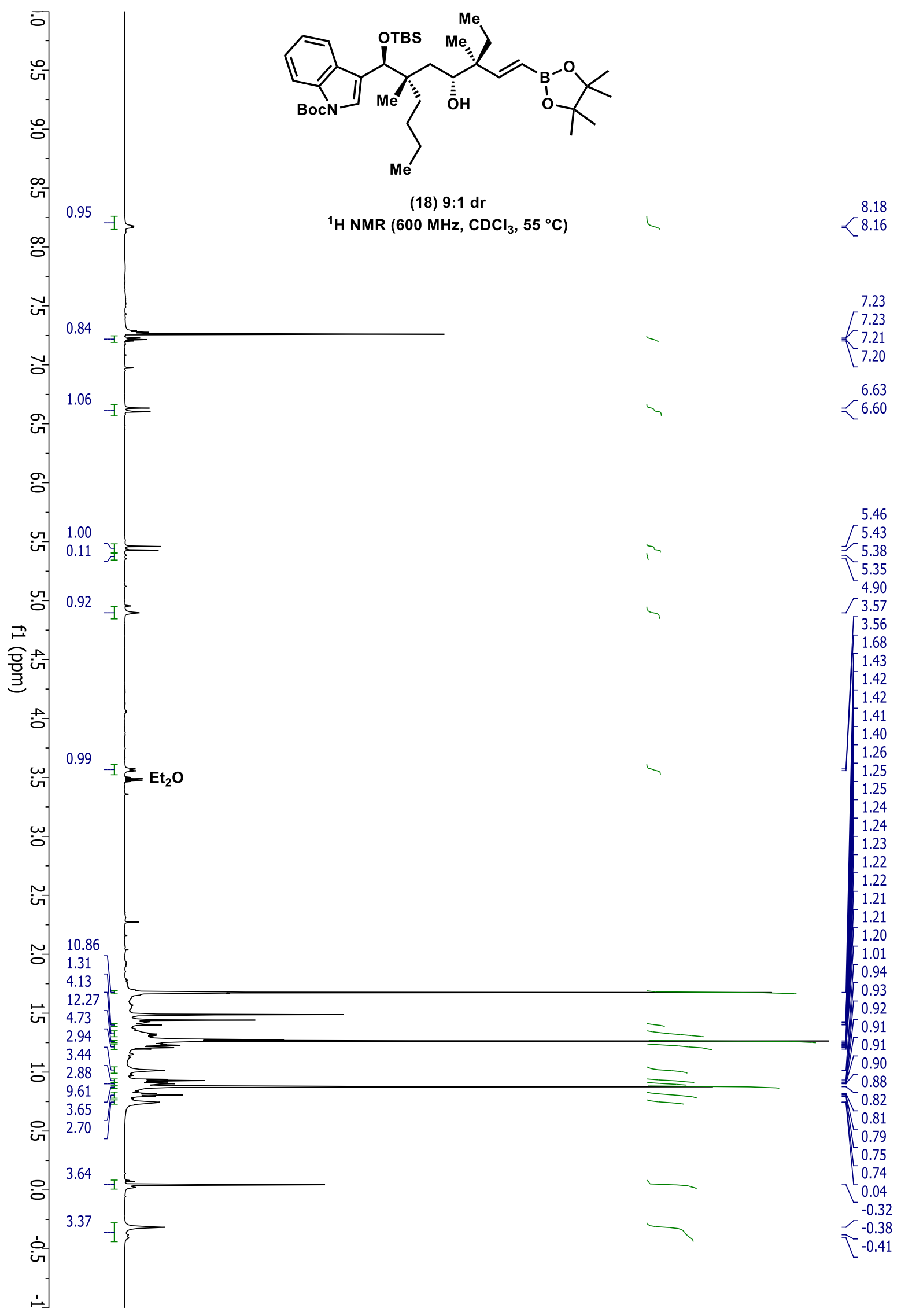




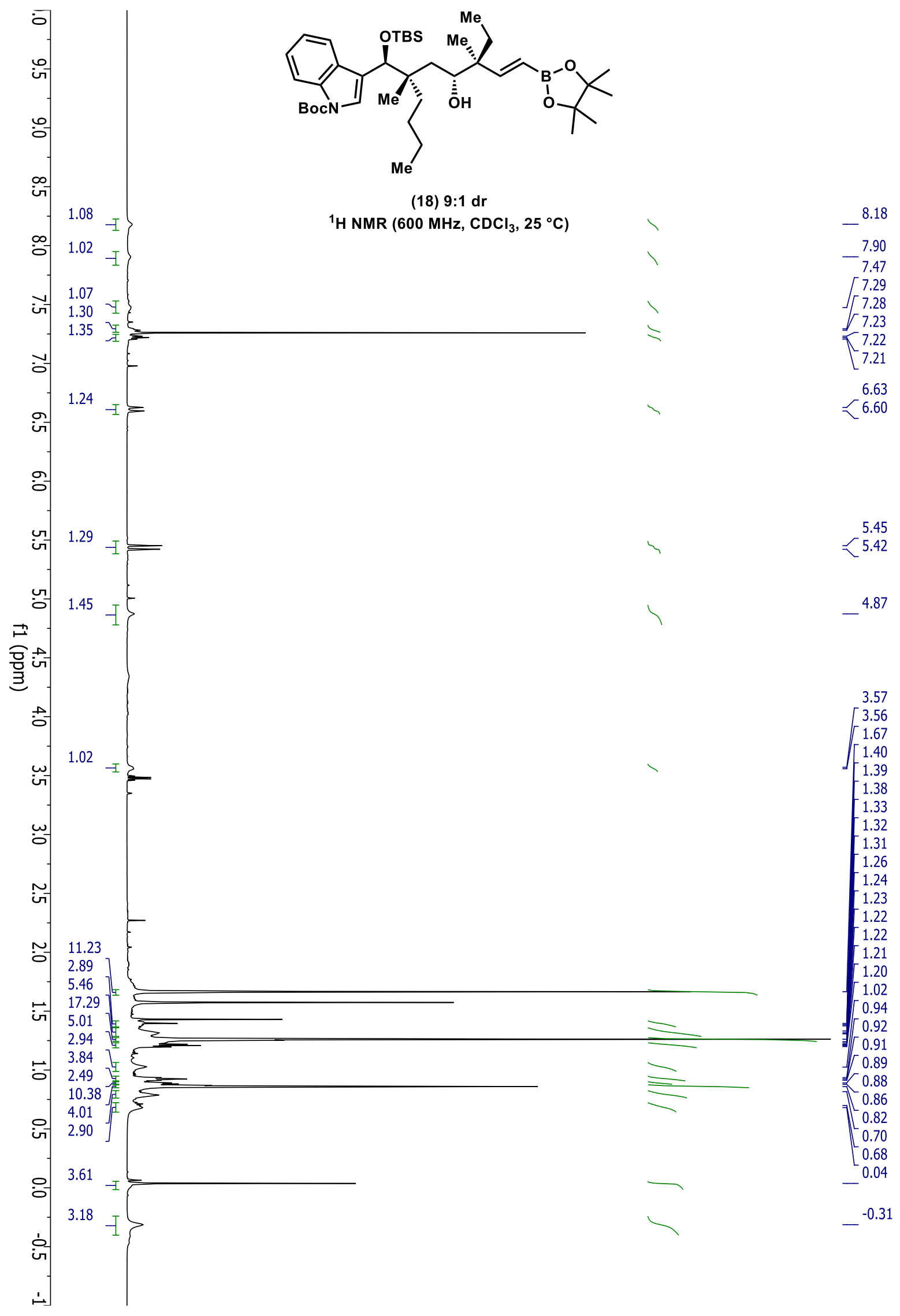




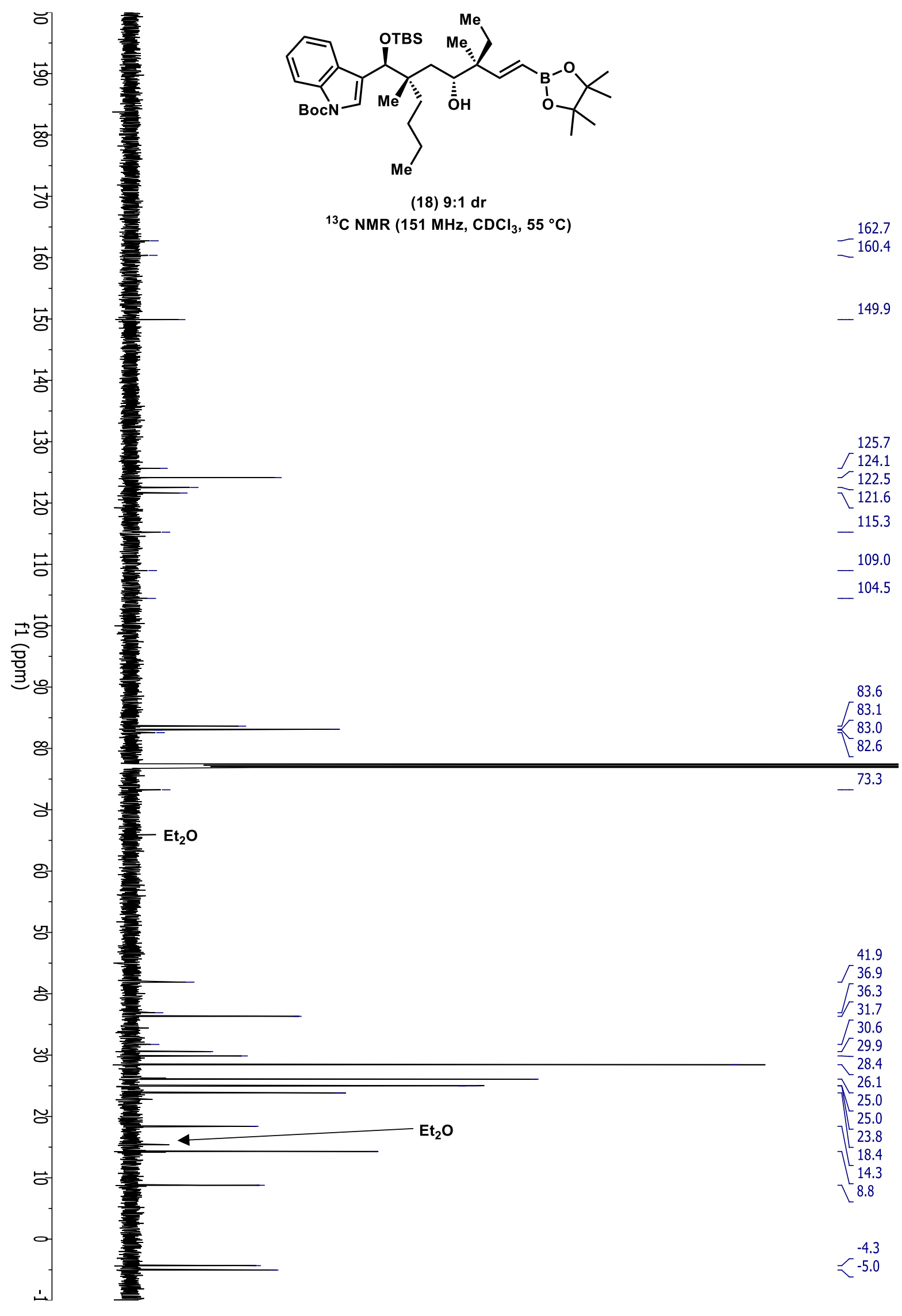




\section{Crystal Data}

Identification code

Empirical formula

Formula weight

Temperature/K

Crystal system

Space group

$\mathrm{a} / \AA$

$\mathrm{b} / \AA$

c/Å

$\alpha /^{\circ}$

$\beta /{ }^{\circ}$

$\gamma^{\circ}$

Volume/ $\AA^{3}$

Z

$\rho_{\text {calc }} / \mathrm{cm}^{3}$

$\mu / \mathrm{mm}^{-1}$

$\mathrm{F}(000)$

Crystal size $/ \mathrm{mm}^{3}$

Radiation
1970642

$\mathrm{C}_{22} \mathrm{H}_{29} \mathrm{BO}_{3} \mathrm{~S}$

384.35

293

monoclinic

$\mathrm{P} 2{ }_{1}$

6.8842(8)

19.284(2)

8.0873(9)

90

93.318(4)

90

1071.8(2)

2

1.191

1.478

412

$0.20 \times 0.20 \times 0.30$

$\mathrm{Cu} \mathrm{K \alpha}(\lambda=1.54178 \AA)$

Index ranges

$-8 \leq \mathrm{h} \leq 8,-22 \leq \mathrm{k} \leq 22,-9 \leq 1 \leq 9$

Reflections collected

16,801

Independent reflections

Data/restraints/parameters

Goodness-of-fit on $\mathrm{F}^{2}$

$3759\left[R_{\text {int }}=0.0002\right]$

$3741 / 342 / 357$

0.999

R-factor

$\mathrm{R}_{1}=0.053, \mathrm{wR}_{2}=0.149$

Flack parameter

$0.04(3)$

Refinement on

F-squared

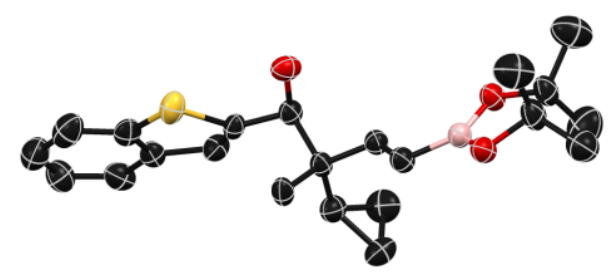

Molecular structure for $6 \mathrm{e}$ (50\% probability level shown)

\section{Parameters}

\begin{tabular}{|l|l|l|l|l|l|}
\hline Atom & $\mathrm{x} / \mathrm{a}$ & $\mathrm{y} / \mathrm{b}$ & $\mathrm{z} / \mathrm{c}$ & $\mathrm{U}(\mathrm{eq})$ & Occupancy \\
\hline S10 & $0.7460(2)$ & $0.60722(9)$ & $0.45840(12)$ & 0.0614 & $0.754(4)$ \\
\hline S101 & $1.1502(6)$ & $0.6719(2)$ & $0.4633(5)$ & $0.0592(13)$ & $0.246(4)$ \\
\hline O1 & $0.6474(3)$ & $0.73081(15)$ & $0.6313(3)$ & 0.0605 & 1 \\
\hline O20 & $0.6182(12)$ & $0.8854(3)$ & $1.1107(4)$ & 0.051 & $0.402(7)$ \\
\hline O21 & $0.6757(8)$ & $0.8897(2)$ & $1.1245(4)$ & 0.0518 & $0.598(7)$ \\
\hline O30 & $0.6338(13)$ & $0.8173(3)$ & $1.3432(6)$ & 0.0485 & $0.402(7)$ \\
\hline O31 & $0.5858(9)$ & $0.81563(18)$ & $1.3266(5)$ & 0.0535 & $0.598(7)$ \\
\hline C1 & $0.7561(4)$ & $0.76077(16)$ & $1.0834(3)$ & 0.0491 & 1 \\
\hline
\end{tabular}




\begin{tabular}{|c|c|c|c|c|c|}
\hline $\mathrm{C} 2$ & $0.8229(4)$ & $0.76547(14)$ & $0.9336(3)$ & 0.0451 & 1 \\
\hline C3 & $0.9103(4)$ & $0.70884(14)$ & $0.8336(3)$ & 0.0437 & 1 \\
\hline $\mathrm{C} 4$ & $0.8394(5)$ & $0.63709(15)$ & $0.8842(3)$ & 0.0553 & 1 \\
\hline $\mathrm{C} 5$ & $1.1316(4)$ & $0.71010(18)$ & $0.8549(3)$ & 0.0563 & 1 \\
\hline C6 & $1.2379(5)$ & $0.7262(2)$ & $1.0169(4)$ & 0.0669 & 1 \\
\hline $\mathrm{C} 7$ & $1.2471(5)$ & $0.7750(2)$ & $0.8763(5)$ & 0.0775 & 1 \\
\hline $\mathrm{C} 8$ & $0.8508(4)$ & $0.72364(15)$ & $0.6477(3)$ & 0.0453 & 1 \\
\hline $\mathrm{C} 11$ & $0.8259(13)$ & $0.6102(6)$ & $0.4533(18)$ & $0.064(5)$ & $0.246(4)$ \\
\hline $\mathrm{C} 90$ & $0.8954(14)$ & $0.6643(7)$ & $0.519(2)$ & $0.038(4)$ & $0.246(4)$ \\
\hline C91 & $0.9234(7)$ & $0.6710(3)$ & $0.5305(7)$ & 0.0431 & $0.754(4)$ \\
\hline $\mathrm{C} 100$ & $1.0850(5)$ & $0.6593(2)$ & $0.4564(5)$ & 0.0551 & $0.754(4)$ \\
\hline C110 & $1.0836(5)$ & $0.59666(18)$ & $0.3474(4)$ & 0.0475 & $0.754(4)$ \\
\hline C111 & $1.1315(10)$ & $0.6083(5)$ & $0.3406(15)$ & $0.044(3)$ & $0.246(4)$ \\
\hline C120 & $1.2304(6)$ & $0.5688(2)$ & $0.2554(6)$ & 0.0643 & $0.754(4)$ \\
\hline C121 & $1.2873(14)$ & $0.5840(8)$ & $0.254(3)$ & $0.075(5)$ & $0.246(4)$ \\
\hline C130 & $1.1850(7)$ & $0.5094(2)$ & $0.1619(5)$ & 0.0661 & $0.754(4)$ \\
\hline C131 & $1.2586(12)$ & $0.5228(5)$ & $0.1633(14)$ & $0.045(3)$ & $0.246(4)$ \\
\hline C140 & $1.0023(7)$ & $0.4807(2)$ & $0.1574(6)$ & 0.0774 & $0.754(4)$ \\
\hline C141 & $1.0836(13)$ & $0.4885(3)$ & $0.1581(10)$ & $0.029(2)$ & $0.246(4)$ \\
\hline C150 & $0.8545(7)$ & $0.5093(2)$ & $0.2466(6)$ & 0.0792 & $0.754(4)$ \\
\hline C151 & $0.9291(12)$ & $0.5120(5)$ & $0.2477(16)$ & $0.045(3)$ & $0.246(4)$ \\
\hline $\mathrm{C} 160$ & $0.9016(5)$ & $0.56754(18)$ & $0.3442(4)$ & 0.0539 & $0.754(4)$ \\
\hline C161 & $0.9599(11)$ & $0.5721(5)$ & $0.3421(16)$ & $0.046(3)$ & $0.246(4)$ \\
\hline C170 & $0.5312(10)$ & $0.8797(3)$ & $1.3878(6)$ & 0.0583 & $0.402(7)$ \\
\hline C171 & $0.5091(7)$ & $0.8827(2)$ & $1.3683(5)$ & 0.0645 & $0.598(7)$ \\
\hline C180 & $0.6208(15)$ & $0.9015(6)$ & $1.5545(9)$ & 0.0809 & $0.402(7)$ \\
\hline C181 & $0.5308(10)$ & $0.8984(4)$ & $1.5516(7)$ & 0.0817 & $0.598(7)$ \\
\hline C190 & $0.3230(13)$ & $0.8626(6)$ & $1.4183(12)$ & 0.1033 & $0.402(7)$ \\
\hline C191 & $0.2934(10)$ & $0.8740(4)$ & $1.3309(9)$ & 0.104 & $0.598(7)$ \\
\hline C200 & $0.5613(10)$ & $0.9299(2)$ & $1.2438(7)$ & 0.0621 & $0.402(7)$ \\
\hline C201 & $0.6113(7)$ & $0.93307(17)$ & $1.2556(5)$ & 0.0608 & $0.598(7)$ \\
\hline C210 & $0.3794(14)$ & $0.9589(6)$ & $1.1599(12)$ & 0.1013 & $0.402(7)$ \\
\hline C211 & $0.4956(11)$ & $0.9952(4)$ & $1.1997(9)$ & 0.0994 & $0.598(7)$ \\
\hline $\mathrm{C} 220$ & $0.6863(19)$ & $0.9915(5)$ & $1.2848(14)$ & 0.1211 & $0.402(7)$ \\
\hline C221 & $0.8025(10)$ & $0.9616(4)$ & $1.3249(9)$ & 0.1036 & $0.598(7)$ \\
\hline B1 & $0.6707(4)$ & $0.82244(13)$ & $1.1788(3)$ & 0.0474 & 1 \\
\hline H11 & 0.7611 & 0.7167 & 1.1362 & 0.0593 & 1 \\
\hline $\mathrm{H} 21$ & 0.8148 & 0.81 & 0.8836 & 0.0545 & 1 \\
\hline H41 & 0.8968 & 0.6026 & 0.8187 & 0.0681 & 1 \\
\hline H42 & 0.8751 & 0.6291 & 0.9979 & 0.0681 & 1 \\
\hline $\mathrm{H} 43$ & 0.7018 & 0.635 & 0.8672 & 0.0681 & 1 \\
\hline H51 & 1.1932 & 0.6753 & 0.7933 & 0.0678 & 1 \\
\hline H61 & 1.1666 & 0.7386 & 1.1096 & 0.0803 & 1 \\
\hline
\end{tabular}




\begin{tabular}{|c|c|c|c|c|c|}
\hline H62 & 1.3496 & 0.7002 & 1.0534 & 0.0803 & 1 \\
\hline H71 & 1.1821 & 0.8184 & 0.8784 & 0.0939 & 1 \\
\hline $\mathrm{H} 72$ & 1.3651 & 0.7801 & 0.8221 & 0.0939 & 1 \\
\hline H81 & 0.9102 & 0.7656 & 0.6153 & 0.0549 & $0.246(4)$ \\
\hline H82 & 0.9056 & 0.7672 & 0.6211 & 0.0549 & $0.754(4)$ \\
\hline H111 & 0.698 & 0.5949 & 0.4722 & 0.0686 & $0.246(4)$ \\
\hline H1001 & 1.1964 & 0.6881 & 0.4727 & 0.0621 & $0.754(4)$ \\
\hline H1201 & 1.3557 & 0.5894 & 0.2548 & 0.0775 & $0.754(4)$ \\
\hline H1211 & 1.4088 & 0.6075 & 0.2569 & 0.0845 & $0.246(4)$ \\
\hline H1301 & 1.2824 & 0.488 & 0.1009 & 0.0787 & $0.754(4)$ \\
\hline H1311 & 1.3615 & 0.5045 & 0.1032 & 0.0551 & $0.246(4)$ \\
\hline H1401 & 0.975 & 0.4404 & 0.0923 & 0.0955 & $0.754(4)$ \\
\hline H1411 & 1.0659 & 0.4481 & 0.0917 & 0.0385 & $0.246(4)$ \\
\hline H1501 & 0.7272 & 0.4902 & 0.2423 & 0.0938 & $0.754(4)$ \\
\hline H1511 & 0.8087 & 0.4879 & 0.2467 & 0.0492 & $0.246(4)$ \\
\hline H1801 & 0.5591 & 0.9428 & 1.5888 & 0.0877 & $0.402(7)$ \\
\hline H1802 & 0.757 & 0.9092 & 1.5527 & 0.0877 & $0.402(7)$ \\
\hline H1803 & 0.5966 & 0.8651 & 1.6297 & 0.0877 & $0.402(7)$ \\
\hline H1811 & 0.4791 & 0.9432 & 1.5704 & 0.0961 & $0.598(7)$ \\
\hline H1812 & 0.664 & 0.8972 & 1.5895 & 0.0961 & $0.598(7)$ \\
\hline H1813 & 0.4605 & 0.8648 & 1.61 & 0.0961 & $0.598(7)$ \\
\hline H1901 & 0.2561 & 0.9035 & 1.4474 & 0.1105 & $0.402(7)$ \\
\hline H1902 & 0.3137 & 0.829 & 1.5032 & 0.1105 & $0.402(7)$ \\
\hline H1903 & 0.2663 & 0.8448 & 1.3172 & 0.1105 & $0.402(7)$ \\
\hline H1911 & 0.2274 & 0.916 & 1.3532 & 0.1127 & $0.598(7)$ \\
\hline H1912 & 0.2458 & 0.8379 & 1.3973 & 0.1127 & $0.598(7)$ \\
\hline H1913 & 0.2714 & 0.8624 & 1.2172 & 0.1127 & $0.598(7)$ \\
\hline H2101 & 0.4199 & 0.989 & 1.0755 & 0.117 & $0.402(7)$ \\
\hline H2102 & 0.3217 & 0.9854 & 1.2432 & 0.117 & $0.402(7)$ \\
\hline H2103 & 0.2871 & 0.9267 & 1.1136 & 0.117 & $0.402(7)$ \\
\hline H2111 & 0.5727 & 1.0226 & 1.1313 & 0.114 & $0.598(7)$ \\
\hline H 2112 & 0.4615 & 1.0216 & 1.2927 & 0.114 & $0.598(7)$ \\
\hline H 2113 & 0.3806 & 0.9809 & 1.1383 & 0.114 & $0.598(7)$ \\
\hline $\mathrm{H} 2201$ & 0.701 & 1.0203 & 1.1909 & 0.1333 & $0.402(7)$ \\
\hline H 2202 & 0.6443 & 1.0188 & 1.3738 & 0.1333 & $0.402(7)$ \\
\hline H2203 & 0.8075 & 0.9705 & 1.3166 & 0.1333 & $0.402(7)$ \\
\hline H2211 & 0.8552 & 0.9919 & 1.2463 & 0.1149 & $0.598(7)$ \\
\hline H 2212 & 0.7831 & 0.9864 & 1.4241 & 0.1149 & $0.598(7)$ \\
\hline H 2213 & 0.8901 & 0.9243 & 1.348 & 0.1149 & $0.598(7)$ \\
\hline $\mathrm{H} 1$ & $0.625(5)$ & $0.7509(18)$ & $0.552(5)$ & 0.05 & 1 \\
\hline
\end{tabular}

\section{Thermal Parameters}




\begin{tabular}{|c|c|c|c|c|c|c|}
\hline Atom & U11 & $\mathrm{U} 22$ & U33 & $\mathrm{U} 23$ & U13 & U12 \\
\hline S10 & $0.0721(9)$ & $0.0681(7)$ & $0.0448(5)$ & $-0.0089(4)$ & $0.0102(5)$ & $-0.0129(5)$ \\
\hline $\mathrm{O} 1$ & $0.0612(11)$ & $0.0775(14)$ & $0.0427(10)$ & $0.0034(10)$ & $0.0028(8)$ & $0.0169(10)$ \\
\hline $\mathrm{O} 20$ & $0.055(4)$ & $0.053(3)$ & $0.045(3)$ & $-0.003(2)$ & $0.003(2)$ & $0.005(2)$ \\
\hline $\mathrm{O} 21$ & $0.064(3)$ & $0.0502(18)$ & $0.0428(17)$ & $-0.0009(14)$ & $0.0129(15)$ & $0.0065(16)$ \\
\hline $\mathrm{O} 30$ & $0.057(3)$ & $0.050(3)$ & $0.038(2)$ & $-0.003(2)$ & $0.004(2)$ & $0.012(2)$ \\
\hline $\mathrm{O} 31$ & $0.064(3)$ & $0.056(2)$ & $0.0409(19)$ & $-0.0014(17)$ & $0.0079(17)$ & $0.0124(18)$ \\
\hline $\mathrm{C} 1$ & $0.0589(14)$ & $0.0476(14)$ & $0.0413(12)$ & $0.0002(11)$ & $0.0077(10)$ & $0.0056(11)$ \\
\hline $\mathrm{C} 2$ & $0.0521(13)$ & $0.0407(12)$ & $0.0425(12)$ & $-0.0005(10)$ & $0.0029(10)$ & $0.0005(10)$ \\
\hline C3 & $0.0566(13)$ & $0.0391(12)$ & $0.0357(12)$ & $-0.0006(9)$ & $0.0057(10)$ & $0.0015(10)$ \\
\hline $\mathrm{C} 4$ & $0.087(2)$ & $0.0397(13)$ & $0.0391(12)$ & $-0.0025(10)$ & $0.0044(12)$ & $0.0008(13)$ \\
\hline C5 & $0.0555(15)$ & $0.0672(18)$ & $0.0463(14)$ & $-0.0087(13)$ & $0.0045(11)$ & $0.0100(13)$ \\
\hline C6 & $0.0586(16)$ & $0.090(2)$ & $0.0515(16)$ & $-0.0010(16)$ & $-0.0020(12)$ & $-0.0030(16)$ \\
\hline $\mathrm{C} 7$ & $0.0647(19)$ & $0.080(2)$ & $0.088(2)$ & $0.012(2)$ & $0.0098(17)$ & $-0.0159(17)$ \\
\hline $\mathrm{C} 8$ & $0.0531(13)$ & $0.0456(13)$ & $0.0376(12)$ & $-0.0010(10)$ & $0.0062(9)$ & $0.0019(10)$ \\
\hline C91 & $0.053(2)$ & $0.044(2)$ & $0.0318(18)$ & $-0.0032(13)$ & $0.0020(17)$ & $-0.0040(16)$ \\
\hline $\mathrm{C} 100$ & $0.0511(14)$ & $0.065(2)$ & $0.0489(14)$ & $-0.0048(14)$ & $-0.0016(15)$ & $-0.003(2)$ \\
\hline C110 & $0.062(2)$ & $0.045(2)$ & $0.0359(17)$ & $0.0064(14)$ & $0.0116(16)$ & $0.0019(18)$ \\
\hline C120 & $0.075(3)$ & $0.070(3)$ & $0.049(2)$ & $0.009(2)$ & $0.017(2)$ & $0.017(3)$ \\
\hline C130 & $0.0689(18)$ & $0.064(3)$ & $0.0674(18)$ & $-0.005(2)$ & $0.021(2)$ & $0.007(2)$ \\
\hline C140 & $0.083(2)$ & $0.081(2)$ & $0.067(3)$ & $-0.013(2)$ & $-0.0038(19)$ & $-0.0035(19)$ \\
\hline $\mathrm{C} 150$ & $0.093(4)$ & $0.075(3)$ & $0.068(3)$ & $0.007(2)$ & $-0.006(3)$ & $-0.031(3)$ \\
\hline C160 & $0.071(3)$ & $0.055(2)$ & $0.0360(19)$ & $0.0089(15)$ & $0.0045(19)$ & $-0.005(2)$ \\
\hline C170 & $0.080(4)$ & $0.054(4)$ & $0.043(3)$ & $-0.002(3)$ & $0.015(3)$ & $0.013(3)$ \\
\hline C171 & $0.085(3)$ & $0.056(3)$ & $0.054(3)$ & $-0.010(2)$ & $0.019(3)$ & $0.013(3)$ \\
\hline C180 & $0.081(3)$ & $0.078(5)$ & $0.081(3)$ & $-0.015(3)$ & $-0.015(3)$ & $0.008(6)$ \\
\hline C181 & $0.085(3)$ & $0.076(4)$ & $0.084(3)$ & $0.002(2)$ & $0.004(2)$ & $0.007(4)$ \\
\hline C190 & $0.098(4)$ & $0.117(7)$ & $0.098(4)$ & $0.034(5)$ & $0.034(5)$ & $0.020(5)$ \\
\hline C191 & $0.097(3)$ & $0.119(6)$ & $0.097(3)$ & $0.015(4)$ & $0.016(4)$ & $0.015(4)$ \\
\hline C200 & $0.084(4)$ & $0.052(4)$ & $0.051(4)$ & $-0.004(3)$ & $0.007(3)$ & $0.012(3)$ \\
\hline C201 & $0.080(3)$ & $0.050(3)$ & $0.053(3)$ & $-0.007(2)$ & $0.016(2)$ & $0.010(2)$ \\
\hline C210 & $0.109(4)$ & $0.109(4)$ & $0.088(5)$ & $0.030(3)$ & $0.016(5)$ & $0.031(3)$ \\
\hline C211 & $0.106(3)$ & $0.104(3)$ & $0.090(4)$ & $0.026(3)$ & $0.020(4)$ & $0.027(3)$ \\
\hline $\mathrm{C} 220$ & $0.130(7)$ & $0.116(4)$ & $0.116(4)$ & $-0.004(4)$ & $-0.003(4)$ & $-0.053(6)$ \\
\hline C221 & $0.102(4)$ & $0.102(4)$ & $0.107(5)$ & $-0.001(2)$ & $0.001(2)$ & $-0.039(4)$ \\
\hline B1 & $0.0565(15)$ & $0.0475(15)$ & $0.0381(13)$ & $-0.0022(12)$ & $0.0027(11)$ & $0.0046(12)$ \\
\hline
\end{tabular}

\section{Distances( $\left(\AA_{)}\right)$}

\begin{tabular}{|c|c|c|c|c|c|}
\hline S10 & C91 & $1.806(4)$ & C121 & C131 & $1.397(2)$ \\
\hline S10 & C160 & $1.643(3)$ & C121 & H1211 & 0.95 \\
\hline S101 & C90 & $1.842(6)$ & C130 & C140 & $1.373(2)$ \\
\hline
\end{tabular}




\begin{tabular}{|c|c|c|c|c|c|}
\hline S101 & C111 & $1.578(6)$ & C130 & H1301 & 0.95 \\
\hline O1 & C8 & $1.405(3)$ & C131 & C141 & $1.374(2)$ \\
\hline $\mathrm{O} 1$ & H1 & $0.76(4)$ & C131 & H1311 & 0.95 \\
\hline $\mathrm{O} 20$ & C200 & $1.448(5)$ & C140 & C150 & $1.395(2)$ \\
\hline $\mathrm{O} 20$ & B1 & $1.374(5)$ & C140 & H1401 & 0.95 \\
\hline $\mathrm{O} 21$ & C201 & $1.440(4)$ & C141 & C151 & $1.397(2)$ \\
\hline $\mathrm{O} 21$ & B1 & $1.371(4)$ & C141 & H1411 & 0.95 \\
\hline $\mathrm{O} 30$ & $\mathrm{C} 170$ & $1.450(5)$ & C150 & C160 & $1.3996(19)$ \\
\hline $\mathrm{O} 30$ & B1 & $1.372(4)$ & C150 & H1501 & 0.95 \\
\hline $\mathrm{O} 31$ & C171 & $1.444(4)$ & C151 & C161 & $1.397(2)$ \\
\hline $\mathrm{O} 31$ & B1 & $1.367(4)$ & C151 & H1511 & 0.95 \\
\hline $\mathrm{C} 1$ & $\mathrm{C} 2$ & $1.324(4)$ & $\mathrm{C} 170$ & C180 & $1.510(6)$ \\
\hline $\mathrm{C} 1$ & B1 & $1.552(3)$ & $\mathrm{C} 170$ & C190 & $1.504(6)$ \\
\hline $\mathrm{C} 1$ & H11 & 0.95 & $\mathrm{C} 170$ & C200 & $1.538(3)$ \\
\hline $\mathrm{C} 2$ & C3 & $1.505(3)$ & C171 & C181 & $1.512(6)$ \\
\hline $\mathrm{C} 2$ & $\mathrm{H} 21$ & 0.95 & C171 & C191 & $1.507(6)$ \\
\hline C3 & $\mathrm{C} 4$ & $1.531(4)$ & C171 & C201 & $1.531(3)$ \\
\hline C3 & C5 & $1.523(4)$ & C180 & H1801 & 0.95 \\
\hline C3 & C8 & $1.562(3)$ & C180 & H1802 & 0.95 \\
\hline $\mathrm{C} 4$ & $\mathrm{H} 41$ & 0.95 & C180 & H1803 & 0.95 \\
\hline $\mathrm{C} 4$ & $\mathrm{H} 42$ & 0.95 & C181 & H1811 & 0.95 \\
\hline $\mathrm{C} 4$ & $\mathrm{H} 43$ & 0.95 & C181 & H1812 & 0.95 \\
\hline C5 & C6 & $1.495(4)$ & C181 & H1813 & 0.95 \\
\hline C5 & $\mathrm{C} 7$ & $1.488(5)$ & C190 & H1901 & 0.95 \\
\hline C5 & H51 & 0.95 & C190 & H1902 & 0.95 \\
\hline C6 & $\mathrm{C} 7$ & $1.481(5)$ & C190 & H1903 & 0.95 \\
\hline C6 & H61 & 0.95 & C191 & H1911 & 0.95 \\
\hline C6 & H62 & 0.95 & C191 & H1912 & 0.95 \\
\hline C7 & $\mathrm{H} 71$ & 0.95 & C191 & H1913 & 0.95 \\
\hline C7 & $\mathrm{H} 72$ & 0.95 & C200 & C210 & $1.498(6)$ \\
\hline C8 & C90 & $1.586(6)$ & C200 & $\mathrm{C} 220$ & $1.494(6)$ \\
\hline $\mathrm{C} 8$ & H81 & 0.95 & C201 & C211 & $1.494(6)$ \\
\hline $\mathrm{C} 8$ & C91 & 1.494(3) & C201 & $\mathrm{C} 221$ & $1.504(6)$ \\
\hline C8 & H82 & 0.95 & $\mathrm{C} 210$ & H2101 & 0.95 \\
\hline $\mathrm{C} 11$ & C90 & $1.254(6)$ & C210 & H2102 & 0.95 \\
\hline C11 & C161 & $1.516(6)$ & $\mathrm{C} 210$ & H2103 & 0.95 \\
\hline $\mathrm{C} 11$ & H111 & 0.95 & C211 & H2111 & 0.95 \\
\hline C91 & C100 & $1.313(4)$ & C211 & H2112 & 0.95 \\
\hline C100 & C110 & $1.495(4)$ & C211 & H2113 & 0.95 \\
\hline C100 & H1001 & 0.95 & C220 & H2201 & 0.95 \\
\hline C110 & C120 & $1.3967(19)$ & $\mathrm{C} 220$ & $\mathrm{H} 2202$ & 0.95 \\
\hline C110 & C160 & $1.3714(19)$ & $\mathrm{C} 220$ & H2203 & 0.95 \\
\hline C111 & C121 & $1.396(2)$ & C221 & H2211 & 0.95 \\
\hline
\end{tabular}




\begin{tabular}{|l|l|l|l|l|l|}
\hline C111 & C161 & $1.373(2)$ & C221 & H2212 & 0.95 \\
\hline C120 & C130 & $1.398(2)$ & C221 & H2213 & 0.95 \\
\hline C120 & H1201 & 0.95 & & & \\
\hline
\end{tabular}

\section{Angles( $\left(^{\circ}\right)$}

\begin{tabular}{|c|c|c|c|c|c|c|c|}
\hline C91 & S10 & C160 & $92.75(8)$ & C140 & $\mathrm{C} 150$ & C160 & $117.21(2)$ \\
\hline C90 & S101 & C111 & $92.83(10)$ & C140 & $\mathrm{C} 150$ & H1501 & 121.8 \\
\hline C8 & $\mathrm{O} 1$ & H1 & $106(3)$ & C160 & $\mathrm{C} 150$ & H1501 & 121 \\
\hline C200 & $\mathrm{O} 20$ & B1 & $107.54(7)$ & C141 & C151 & C161 & 117.19(3) \\
\hline C201 & $\mathrm{O} 21$ & B1 & 107.37(7) & C141 & C151 & H1511 & 121.8 \\
\hline C170 & $\mathrm{O} 30$ & B1 & $107.56(7)$ & C161 & C151 & H1511 & 121 \\
\hline C171 & $\mathrm{O} 31$ & B1 & $107.40(7)$ & $\mathrm{C} 150$ & C160 & C110 & $121.38(2)$ \\
\hline $\mathrm{C} 2$ & $\mathrm{C} 1$ & $\mathrm{~B} 1$ & $124.4(2)$ & $\mathrm{C} 150$ & $\mathrm{C} 160$ & S10 & $123.59(8)$ \\
\hline $\mathrm{C} 2$ & $\mathrm{C} 1$ & $\mathrm{H} 11$ & 117.8 & C110 & C160 & S10 & $115.02(8)$ \\
\hline B1 & $\mathrm{C} 1$ & H11 & 117.8 & C11 & C161 & C151 & $130.43(11)$ \\
\hline $\mathrm{C} 1$ & $\mathrm{C} 2$ & $\mathrm{C} 3$ & $127.8(2)$ & $\mathrm{C} 11$ & C161 & C111 & $108.18(10)$ \\
\hline $\mathrm{C} 1$ & $\mathrm{C} 2$ & $\mathrm{H} 21$ & 115.9 & C151 & C161 & C111 & $121.36(3)$ \\
\hline C3 & $\mathrm{C} 2$ & $\mathrm{H} 21$ & 116.3 & $\mathrm{O} 30$ & $\mathrm{C} 170$ & C180 & $105.9(6)$ \\
\hline $\mathrm{C} 2$ & $\mathrm{C} 3$ & $\mathrm{C} 4$ & $111.6(2)$ & $\mathrm{O} 30$ & $\mathrm{C} 170$ & C190 & $110.0(6)$ \\
\hline $\mathrm{C} 2$ & $\mathrm{C} 3$ & $\mathrm{C} 5$ & $110.9(2)$ & $\mathrm{C} 180$ & $\mathrm{C} 170$ & C190 & $105.00(7)$ \\
\hline $\mathrm{C} 4$ & $\mathrm{C} 3$ & $\mathrm{C} 5$ & $108.5(2)$ & $\mathrm{O} 30$ & C170 & $\mathrm{C} 200$ & 104.16(7) \\
\hline $\mathrm{C} 2$ & $\mathrm{C} 3$ & $\mathrm{C} 8$ & $107.08(19)$ & C180 & $\mathrm{C} 170$ & $\mathrm{C} 200$ & $115.73(7)$ \\
\hline $\mathrm{C} 4$ & $\mathrm{C} 3$ & $\mathrm{C} 8$ & $110.6(2)$ & C190 & $\mathrm{C} 170$ & $\mathrm{C} 200$ & $115.65(7)$ \\
\hline C5 & $\mathrm{C} 3$ & $\mathrm{C} 8$ & 108.1(2) & $\mathrm{O} 31$ & $\mathrm{C} 171$ & $\mathrm{C} 181$ & $113.1(4)$ \\
\hline C3 & $\mathrm{C} 4$ & $\mathrm{H} 41$ & 109.5 & $\mathrm{O} 31$ & $\mathrm{C} 171$ & C191 & $102.9(4)$ \\
\hline $\mathrm{C} 3$ & $\mathrm{C} 4$ & $\mathrm{H} 42$ & 109.6 & C181 & $\mathrm{C} 171$ & C191 & $105.04(7)$ \\
\hline $\mathrm{H} 41$ & $\mathrm{C} 4$ & $\mathrm{H} 42$ & 109.5 & $\mathrm{O} 31$ & C171 & C201 & $104.01(7)$ \\
\hline C3 & $\mathrm{C} 4$ & $\mathrm{H} 43$ & 109.3 & C181 & C171 & $\mathrm{C} 201$ & $115.69(7)$ \\
\hline $\mathrm{H} 41$ & $\mathrm{C} 4$ & $\mathrm{H} 43$ & 109.5 & C191 & C171 & C201 & 115.61(7) \\
\hline $\mathrm{H} 42$ & $\mathrm{C} 4$ & $\mathrm{H} 43$ & 109.5 & $\mathrm{C} 170$ & C180 & H1801 & 109.1 \\
\hline $\mathrm{C} 3$ & $\mathrm{C} 5$ & C6 & $122.5(2)$ & $\mathrm{C} 170$ & $\mathrm{C} 180$ & H1802 & 112.5 \\
\hline C3 & $\mathrm{C} 5$ & $\mathrm{C} 7$ & $123.4(3)$ & H1801 & C180 & H1802 & 109.5 \\
\hline C6 & $\mathrm{C} 5$ & $\mathrm{C} 7$ & $59.5(2)$ & $\mathrm{C} 170$ & C180 & H1803 & 106.7 \\
\hline C3 & $\mathrm{C} 5$ & H51 & 113.8 & H1801 & C180 & H1803 & 109.5 \\
\hline C6 & $\mathrm{C} 5$ & H51 & 113.3 & $\mathrm{H} 1802$ & C180 & H1803 & 109.5 \\
\hline C7 & $\mathrm{C} 5$ & H51 & 113.8 & C171 & C181 & H1811 & 108.8 \\
\hline C5 & C6 & $\mathrm{C} 7$ & $60.0(2)$ & $\mathrm{C} 171$ & C181 & H1812 & 110.5 \\
\hline C5 & C6 & H61 & 119.6 & H1811 & $\mathrm{C} 181$ & H1812 & 109.5 \\
\hline C7 & C6 & H61 & 119.5 & C171 & C181 & H1813 & 109.1 \\
\hline C5 & C6 & H62 & 120.6 & H1811 & C181 & H1813 & 109.5 \\
\hline
\end{tabular}




\begin{tabular}{|c|c|c|c|c|c|c|c|}
\hline C7 & C6 & H62 & 120.3 & H1812 & C181 & H1813 & 109.5 \\
\hline H61 & C6 & H62 & 109.5 & C170 & C190 & H1901 & 109.9 \\
\hline C5 & $\mathrm{C} 7$ & C6 & $60.5(2)$ & C170 & C190 & H1902 & 111.9 \\
\hline C5 & C7 & H71 & 119.7 & H1901 & C190 & H1902 & 109.5 \\
\hline C6 & $\mathrm{C} 7$ & $\mathrm{H} 71$ & 120.4 & $\mathrm{C} 170$ & C190 & H1903 & 106.6 \\
\hline C5 & C7 & $\mathrm{H} 72$ & 119.9 & H1901 & C190 & H1903 & 109.5 \\
\hline C6 & C7 & $\mathrm{H} 72$ & 119.7 & H1902 & C190 & H1903 & 109.5 \\
\hline H71 & $\mathrm{C} 7$ & $\mathrm{H} 72$ & 109.5 & C171 & C191 & H1911 & 110.1 \\
\hline C3 & C8 & $\mathrm{O} 1$ & $108.3(2)$ & C171 & C191 & H1912 & 109.6 \\
\hline C3 & C8 & C90 & $116.6(9)$ & H1911 & C191 & H1912 & 109.5 \\
\hline $\mathrm{O} 1$ & C8 & C90 & $103.8(4)$ & C171 & C191 & H1913 & 108.7 \\
\hline C3 & C8 & H81 & 109.2 & H1911 & C191 & H1913 & 109.5 \\
\hline $\mathrm{O} 1$ & C8 & H81 & 109.4 & H1912 & C191 & H1913 & 109.5 \\
\hline C90 & C8 & H81 & 109.3 & C170 & C200 & $\mathrm{O} 20$ & $104.15(7)$ \\
\hline C3 & C8 & $\mathrm{O} 1$ & $108.3(2)$ & C170 & C200 & C210 & $115.64(7)$ \\
\hline C3 & C8 & C91 & 114.1(3) & $\mathrm{O} 20$ & C200 & $\mathrm{C} 210$ & $98.1(6)$ \\
\hline $\mathrm{O} 1$ & C8 & C91 & $112.0(3)$ & $\mathrm{C} 170$ & C200 & $\mathrm{C} 220$ & $115.72(7)$ \\
\hline C3 & C8 & H82 & 107.1 & $\mathrm{O} 20$ & C200 & C220 & 117.3(5) \\
\hline $\mathrm{O} 1$ & C8 & H82 & 107.3 & C210 & C200 & $\mathrm{C} 220$ & $105.00(7)$ \\
\hline C91 & C8 & H82 & 107.7 & C171 & C201 & $\mathrm{O} 21$ & $103.99(7)$ \\
\hline C90 & C11 & C161 & $115.21(10)$ & C171 & C201 & C211 & $115.68(7)$ \\
\hline C90 & C11 & H111 & 121.7 & $\mathrm{O} 21$ & C201 & $\mathrm{C} 211$ & 115.1(4) \\
\hline C161 & C11 & H111 & 123.1 & $\mathrm{C} 171$ & C201 & $\mathrm{C} 221$ & $115.66(7)$ \\
\hline S101 & C90 & C8 & 109.1(4) & $\mathrm{O} 21$ & C201 & C221 & $100.8(4)$ \\
\hline S101 & C90 & C11 & $107.84(10)$ & C211 & C201 & C221 & $105.03(7)$ \\
\hline C8 & C90 & $\mathrm{C} 11$ & $142.6(5)$ & C200 & C210 & H2101 & 106.4 \\
\hline $\mathrm{C} 8$ & C91 & S10 & $115.0(2)$ & C200 & C210 & H2102 & 104.6 \\
\hline $\mathrm{C} 8$ & C91 & C100 & $136.9(3)$ & H2101 & C210 & H2102 & 109.5 \\
\hline S10 & C91 & $\mathrm{C} 100$ & $108.06(8)$ & C200 & C210 & H2103 & 117.2 \\
\hline C91 & C100 & $\mathrm{C} 110$ & $115.56(9)$ & H2101 & C210 & $\mathrm{H} 2103$ & 109.5 \\
\hline C91 & C100 & H1001 & 122.3 & H2102 & C210 & H2103 & 109.5 \\
\hline C110 & C100 & H1001 & 122.1 & C201 & C211 & H2111 & 108.5 \\
\hline C100 & $\mathrm{C} 110$ & C120 & 130.13(9) & C201 & C211 & H2112 & 110 \\
\hline C100 & $\mathrm{C} 110$ & $\mathrm{C} 160$ & $108.47(8)$ & H2111 & C211 & $\mathrm{H} 2112$ & 109.5 \\
\hline C120 & C110 & C160 & $121.38(2)$ & C201 & C211 & $\mathrm{H} 2113$ & 109.9 \\
\hline S101 & C111 & $\mathrm{C} 121$ & $122.7(3)$ & H2111 & C211 & H2113 & 109.5 \\
\hline S101 & C111 & C161 & $115.36(9)$ & H2112 & C211 & $\mathrm{H} 2113$ & 109.5 \\
\hline C121 & C111 & C161 & $121.37(3)$ & C200 & $\mathrm{C} 220$ & H2201 & 111.9 \\
\hline C110 & C120 & C130 & 117.21(2) & C200 & $\mathrm{C} 220$ & H2202 & 114.4 \\
\hline C110 & C120 & H1201 & 121.8 & H2201 & C220 & H2202 & 109.5 \\
\hline C130 & C120 & H1201 & 121 & C200 & $\mathrm{C} 220$ & H2203 & 101.8 \\
\hline C111 & C121 & C131 & $117.20(3)$ & H2201 & $\mathrm{C} 220$ & H2203 & 109.5 \\
\hline C111 & C121 & H1211 & 121.8 & H2202 & $\mathrm{C} 220$ & H2203 & 109.5 \\
\hline
\end{tabular}




\begin{tabular}{|l|l|l|l|l|l|l|l|}
\hline C131 & C121 & H1211 & 121 & C201 & C221 & H2211 & 109.6 \\
\hline C120 & C130 & C140 & $121.39(2)$ & C201 & C221 & H2212 & 109.7 \\
\hline C120 & C130 & H1301 & 119.7 & H2211 & C221 & H2212 & 109.5 \\
\hline C140 & C130 & H1301 & 118.9 & C201 & C221 & H2213 & 109.1 \\
\hline C121 & C131 & C141 & $121.37(3)$ & H2211 & C221 & H2213 & 109.5 \\
\hline C121 & C131 & H1311 & 119.7 & H2212 & C221 & H2213 & 109.5 \\
\hline C141 & C131 & H1311 & 118.9 & C1 & B1 & O20 & $125.2(2)$ \\
\hline C130 & C140 & C150 & $121.39(2)$ & C1 & B1 & O30 & $121.6(2)$ \\
\hline C130 & C140 & H1401 & 119.7 & O20 & B1 & O30 & $113.17(7)$ \\
\hline C150 & C140 & H1401 & 118.9 & C1 & B1 & O21 & $123.3(2)$ \\
\hline C131 & C141 & C151 & $121.37(3)$ & C1 & B1 & O31 & $123.69(19)$ \\
\hline C131 & C141 & H1411 & 119.7 & O21 & B1 & O31 & $113.03(7)$ \\
\hline C151 & C141 & H1411 & 118.9 & & & & \\
\hline
\end{tabular}




\section{References}

1 J. C. Green, M.V. Joannou, S. A. Murray, J. M. Zanghi, S. J. Meek, ACS Catal. 2017, 7, 4441 - 4445.

2 J. M. Zanghi, S. J. Meek, Angew. Chem. Int. Ed. 2020, 59, $8451-8455$.

3 J. C. Green, J. M. Zanghi, S. J. Meek, J. Am. Chem. Soc. 2020, 142, $1704-1709$.

${ }^{4}$ K. Junge, B. Wendt, S. Enthaler, M. Beller, ChemCatChem. 2010, 2, $453-458$.

${ }^{5}$ S. Brenet, C. Minozzi, B. Clarens, L. Amiri, F. Berthiol, Synthesis. 2015, 47, 3859 - 3873.

${ }^{6}$ G. A. Kraus, M. T. Molina, J. A. Walling, J. Chem. Soc., Chem. Commun. 1986, 1568 - 1569. 